\title{
Integrating microeconomics and social psychology - the case of discrimination in the labor market
}

Citation for published version (APA):

Schwieren, C. A. A. (2003). Integrating microeconomics and social psychology - the case of discrimination in the labor market. [Doctoral Thesis, Maastricht University]. Universiteit Maastricht. https://doi.org/10.26481/dis.20030912cs

Document status and date:

Published: 01/01/2003

DOI:

10.26481/dis.20030912cs

Document Version:

Publisher's PDF, also known as Version of record

\section{Please check the document version of this publication:}

- A submitted manuscript is the version of the article upon submission and before peer-review. There can be important differences between the submitted version and the official published version of record.

People interested in the research are advised to contact the author for the final version of the publication, or visit the DOI to the publisher's website.

- The final author version and the galley proof are versions of the publication after peer review.

- The final published version features the final layout of the paper including the volume, issue and page numbers.

Link to publication

\footnotetext{
General rights rights.

- You may freely distribute the URL identifying the publication in the public portal. please follow below link for the End User Agreement:

www.umlib.nl/taverne-license

Take down policy

If you believe that this document breaches copyright please contact us at:

repository@maastrichtuniversity.nl

providing details and we will investigate your claim.
}

Copyright and moral rights for the publications made accessible in the public portal are retained by the authors and/or other copyright owners and it is a condition of accessing publications that users recognise and abide by the legal requirements associated with these

- Users may download and print one copy of any publication from the public portal for the purpose of private study or research.

- You may not further distribute the material or use it for any profit-making activity or commercial gain

If the publication is distributed under the terms of Article $25 \mathrm{fa}$ of the Dutch Copyright Act, indicated by the "Taverne" license above, 


\section{Integrating microeconomics and social psychology - the case of discrimination in the labor market}





\section{Integrating microeconomics and social psychology - the case of discrimination in the labor market}

\section{PROEFSCHRIFT}

ter verkrijging van de graad van doctor aan de Universiteit Maastricht, op gezag van de Rector Magnificus, Prof. Dr. A.C. Nieuwenhuijzen Kruseman, volgens het besluit van het College van Decanen, in het openbaar te verdedigen op vrijdag 12 september 2003 om 12:00 uur

door

Christiane Schwieren 


\section{Promotor:}

Prof. dr. P.P. de Gijsel

Co-promotor:

Dr. M.C.M. Vendrik

\section{Beoordelingscommissie:}

Prof. dr. C.J.N. de Neubourg (voorzitter)

Prof. dr. J. Brandts (Institut d'Anàlisi Econòmica, Barcelona, Spain)

Prof. dr. P.P. Wakker 


\section{Foreword}

When I came to Maastricht four years ago, a cultural change in two ways expected me - from Germany to the Netherlands and to Belgium, where 1 live, and from a psychology department to an economics department. Noticing the cultural differences between Germany, the Netherlands, and Belgium made me more aware about many things typical for my home country, which I never realized as such before.

During the first two years 1 resisted to turn into an economist, visited doctoral workshops in social psychology and spent a lot of time discussing my research with social psychologists. I learned a lot about economics from my supervisors and my colleagues, but I never really liked economics. My stay in Barcelona at Universitat Autonoma changed this. In many discussions with economists working interdisciplinary or being interested in interdisciplinary research, I finally understood the values of economic thinking. The time in Barcelona, of course was not only research-wise a nice experience. Last but not least, the stay resulted in a new double-cultural change for the time to come - my next job will be at Universitat Pompeu Fabra in Barcelona, as an assistant professor in Business.

For contributing to my identity-loss as a psychologist 1 first and foremost have to thank my supervisors, Maarten Vendrik and Peter de Gijsel. They gave me the possibility to work independently, and encouraged me to go to many summer schools and conferences, most of them interdisciplinary, which helped to build a network of contacts in experimental economics and economic psychology. Peter made me think over my ideas again and again, playing the devil's advocate, questioning all I was doing - but always in a useful way which led to an improvement of my results. Maarten on the other hand had a quite hard time teaching me to be precise and to value good structure. He did not really turn me into a perfectionist, but pushed me a bit further in that direction. I also want to thank Arien van Witteloostuijn and Christophe Boone who helped me in the beginning to define my project, and encouraged me to find my own way to do research.

I also want to thank my colleague, friend, and 'paranymphe' Franziska Gassmann. With her I shared the office in the beginning and we became friends quite quickly. She told me all I needed to know to get a first understanding of this university, of Maastricht, and of the Netherlands. My second 'paranymphe', Ursula Glunk, made me 
aware of the field of Organizational Behavior, which is now becoming my new area of research in Barcelona. Doing research and going to several conferences together however is a secondary aspect of our friendship.

For contributions to one or several of the chapters I want to thank among others especially the following people: For helpful discussions and comments about the theoretical model I owe thank to Lex Borghans, Pablo Guillen Alvarez, Rein Haagsma, Georg Kirchsteiger, Peter Wakker, Geert Woltjer, and the participants of a NAKE Research Day in Amsterdam and the MiMaMa seminar at the Universiteit Maastricht. For helping with the data collection in Heidelberg I want to thank Tilmann Betsch, Susanne Haberstroh, Henning Plessner and several student assistants. Pia Steffens helped with the experiments in Maastricht. Joep Sonnemans, Jos Theelen, Jens Grosser, and other members of the CREED-team in Amsterdam provided most valuable help with the computerized experiments. I also want to thank Michael Huke for hints regarding programming, and Armin Falk for allowing me to use one of his programs and giving me advise on z-tree whenever needed. Ineke Verhagen helped with the data collection during both management games, and Rita Walczuch, Jan Nijhuis and several tutors supported me in convincing students to participate, and more general in conducting the research. I would also like to thank David Rodriguez for his great help in preparing and executing the double auction experiment, and Jordi Brandts and Marc Vorsatz for translating the originally German instructions into Spanish. Moreover, I would like to thank Matthias Sutter and Martin Strobel for useful comments on a draft of the paper chapter 6 is based on.

Writing a thesis and starting an academic career is not only a matter of colleagues, but you also need the support of friends and family. I therefore want to thank my parents for all their support during my long education, and for always stimulating my intellectual interests. Last but not least, I want to thank my partner Michael for all his support, for sustaining my periods of being doubtful about what I was doing, and for telling me to stop ruminating and 'cut and eat' when necessary. Apart from emotional support, he helped a lot with all computer-related aspects of my work (thanks to him my laptop contains a cork of a bottle of wine), and also with structuring and presenting my work. 


\section{Contents}

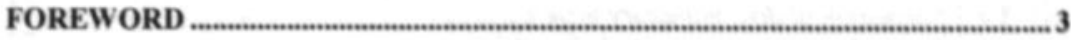

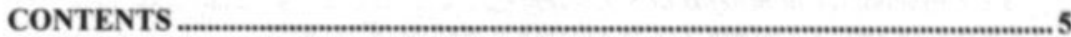

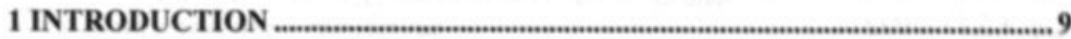

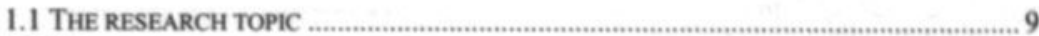

1.2 ECONOMIC AND PSYCHOLOGICAL APPROACHES TO DISCRIMINATION .......................11

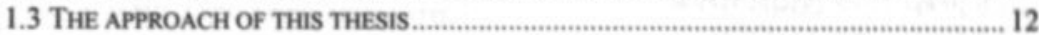

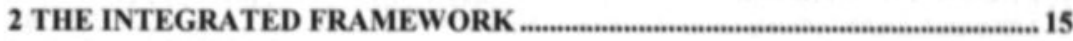

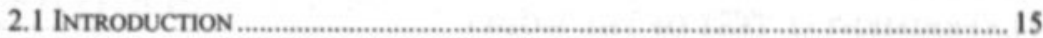

2.2 EMPIRICAL EVIDENCE FOR DISCRIMINATION IN THE LABOR MARKET ..................... 17

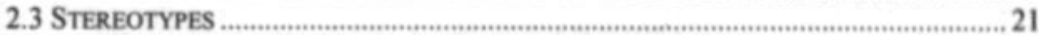

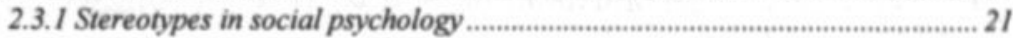

2.3.2 Statistical discrimination - stereotypes in economics .......................................27

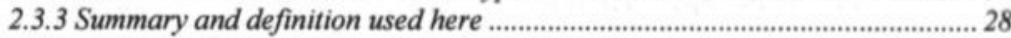

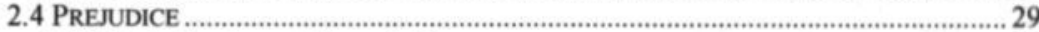

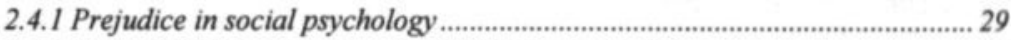

2.4.2 Tastes for discrimination - prejudice in economics.......................................... 31

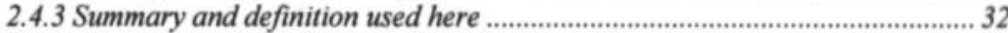

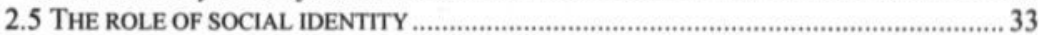

2.5.1 The link between cognition, emotion, and behavior ......................................... 33

2.5.2 Social identity theory and self-categorization theory........................................34

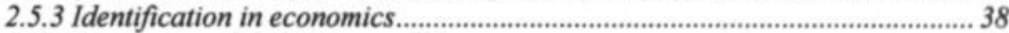

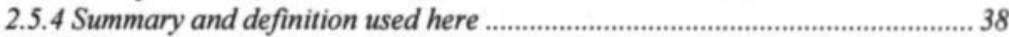

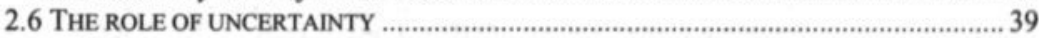

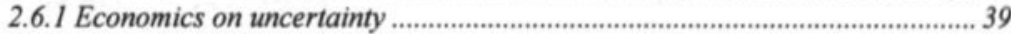

2.6.2 Social psychology on uncertainty ................................................................ 42

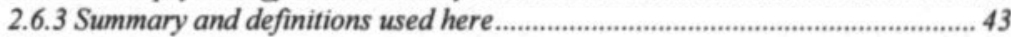

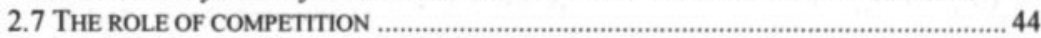

2.7.1 Economics on the role of competition .............................................................. 44

2.7.2 Social psychology on the role of competition ...................................................49

2.7.3 Summary and definition used here ................................................................ 49

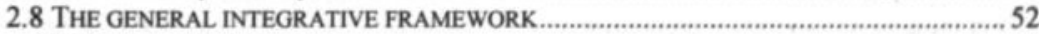

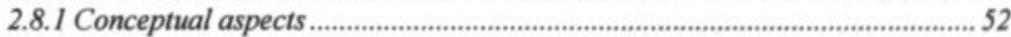

2.8.2 Methodological questions .......................................................................5

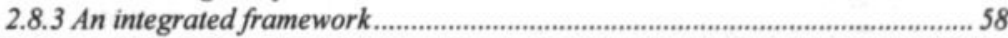

3 AN INTEGRATED MODEL .......................................................................................62

3.1 INTRODUCTION

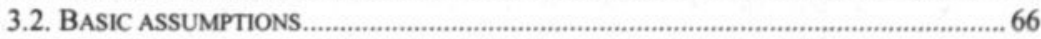

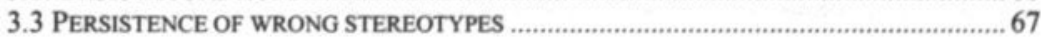


3.4 BASIC MODEL: PSYCHOLOGICAL BENEFITS ......................................................... 68

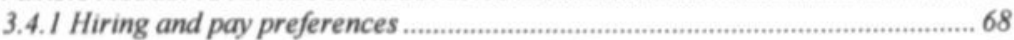

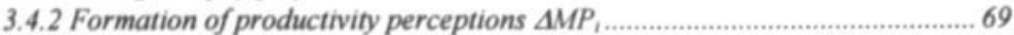

3.4.3 Formation of discriminatory taste $\varepsilon$.............................................................. 71

3.4.4 Psychological bene fits of S, I, T and C......................................................... 72

3.5 BASIC MODEL: ECONOMIC COSTS AND EQUILIBRIUM OUTCOMES …............................ 75

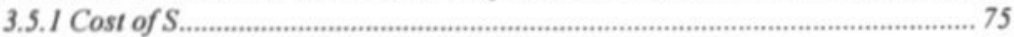

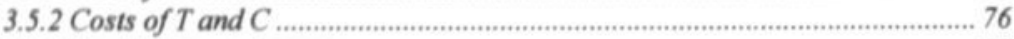

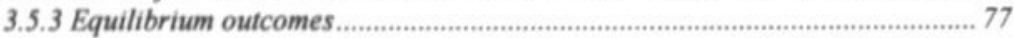

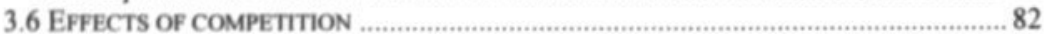

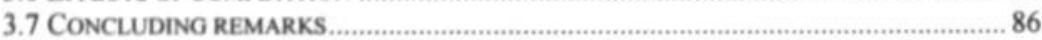

APPENDIX: DERIVATIONS OF EFFECTS OF COMPETITION .............................................. 87

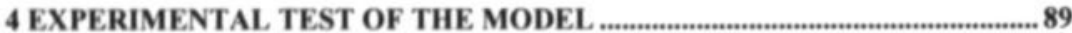

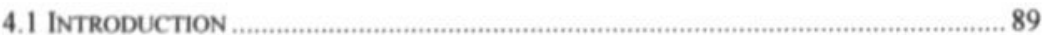

4.2 GENERAL DESIGN, LINK WITH THE THEORETICAL MODEL, AND HYPOTHESES ..........91

4.2.I General design................................................................................................... 9]

4.2.2 Link with the theoretical model ........................................................................ 94

4.2.3 The hypotheses................................................................................................... 99

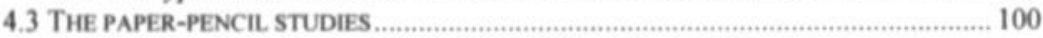

4.3.I Subjects .................................................................................................... 100

4.3.2 Material .................................................................................................. 101

4.3.3 Procedure ............................................................................................. 103

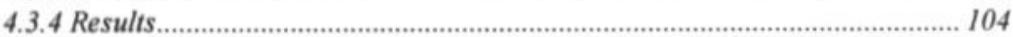

4.3.5 Discussion ...................................................................................................... 107

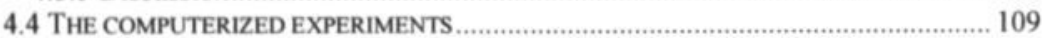

4.4.1 Experiment 3 ............................................................................................ 110

4.4.2 Experiment 4............................................................................................... 120

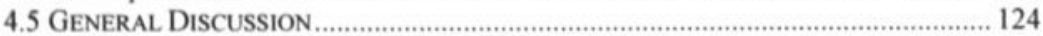

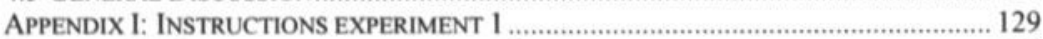

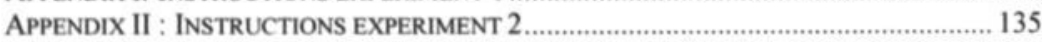

APPENDIX III : INSTRUCTIONS EXPERIMENTS $3 \&$ \& ................................................. 141

5 AN EMPIRICAL TEST IN A BUSINESS-SIMULATION GAME .................... 144

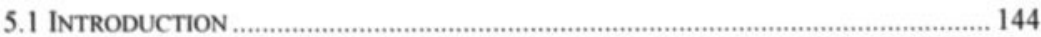

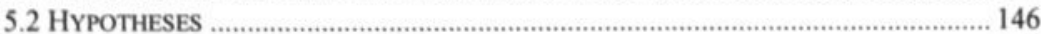

5.3 DESIGN OF THE QUASI-EXPERIMENT ….......................................................... 150

5.3.1 The management game ................................................................................... 150

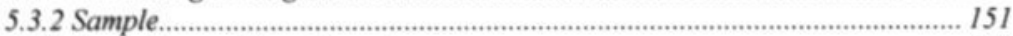

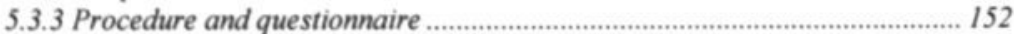

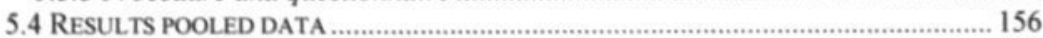

5.4.1 Hypothesis Ia) - main effect of nationality ..................................................... 156

5.4.2 Hypothesis Ib) (main effect psycho-economic mechanism) .......................... 157

5.4.3 Hypothesis Ic) (interaction effect psychological mechanism) ..................... I57 
5.4.4 Hypothesis 2a) ('statistical' discrimination).

5.4.5 Hypothesis 2b)(psychological effect of competition).................................... I59

5.4 .6 Exploratory analysis................................................................................... 160

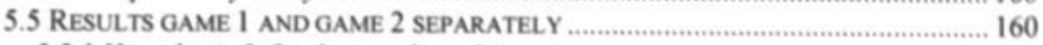

5.5.I Hypothesis 3 (background conditions) ....................................................... 160

5.5.2 Exploratory analyses for the two games separately ................................... 16I

5.6 DiSCUSSION AND CONCLUSION ......................................................................... 163

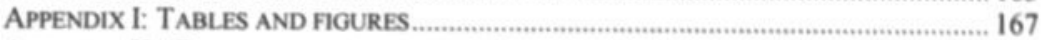

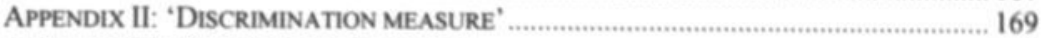

APPENDIX III : QUESTIONNAIRE GAME 1 ......................................................... 170

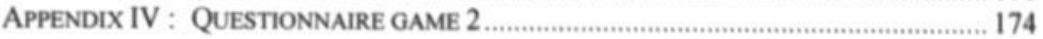

6 IS IT EFFICIENCY WAGES?

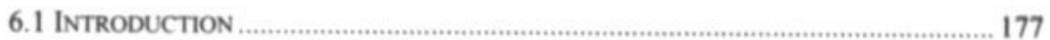

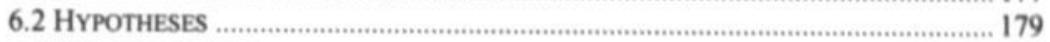

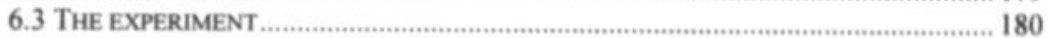

6.3.1 Participants.............................................................................................. $/ 80$

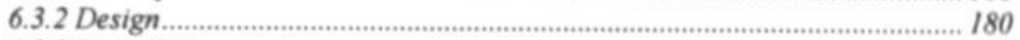

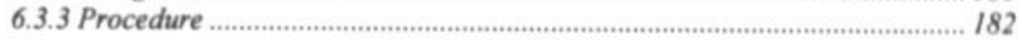

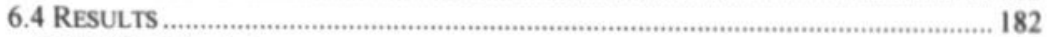

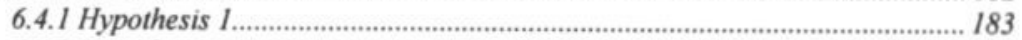

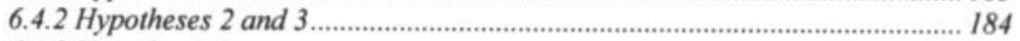

6.4 .3 Hypothesis 4 ............................................................................................ 187

6.4 .4 Other results ............................................................................................... 188

6.4.5 The mixed sessions.................................................................................... 191

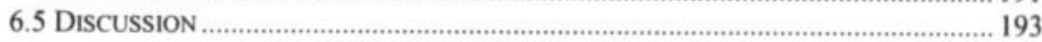

APPENDIX: INSTRUCTIONS TRANSLATED INTO ENGLISH ........................................ 197

7 SUMMARY, GENERAL DISCUSSION, AND CONCLUSIONS........................ 211

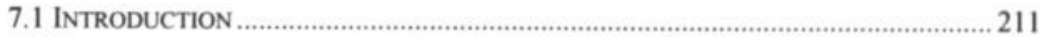

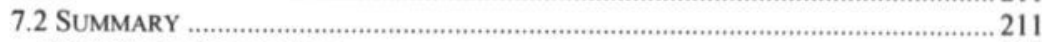

7.2.1 The basic questions......................................................................................... 21]

7.2.2 Short summary of each chapter ...................................................................... 21I

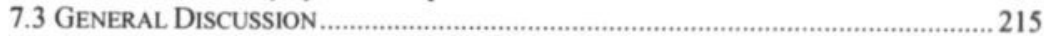

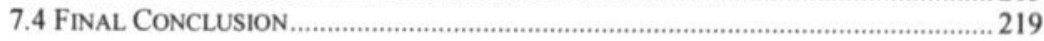

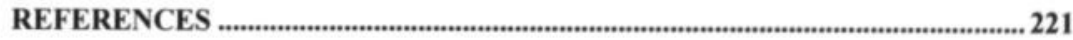

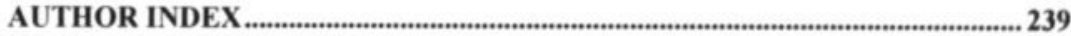

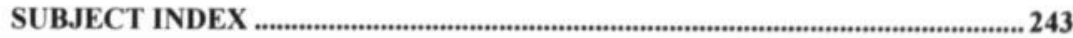

SUMMARY

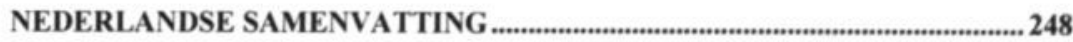

ABOUT THE AUTHOR ........................................................................................... 252 
Contents 


\section{Introduction}

\subsection{The research topic}

The last decade has seen a growing interest of economics in social- and cognitivepsychological research. This interest has evolved from empirical findings showing that people in 'real-life' often do not behave in line with the assumptions of standard economic theory. Other factors than those considered so far in mainstream economics have been found to influence behavior, like preferences for faimess (e.g., Fehr \& Schmidt, 1999), the willingness to punish others for lying (Brandts \& Charness, 2003), impatience (Laibson, 1997), 'social ties' (Granovetter, 1973; van Dijk, Sonnemans, van Winden, 2002), heuristic decision-making (Gigerenzer \& Selten, 2001) or limited willpower (O'Donoghue \& Rabin, 1999). Using such concepts, many kinds of behavior found in real-life situations could be explained better, and could also be reproduced in the controlled setting of experiments.

This thesis adopts the idea of integrating social-psychological and economic theorizing to approach a complex, politically and economically relevant problem: Discrimination in the labor market. Because of its surprising persistence even in industrialized societies, which actively strive to eliminate it, a lot of research in economics, (social-) psychology, and related disciplines deals with discrimination (in the labor market). Apart from providing evidence of its existence, using mainly econometric methods (e.g. recently Blau \& Kahn, 2000; see section 2.2 for an overview of empirical economic literature), economic research of various theoretical orientations tries to explain the phenomenon and its persistence (see sections $1.2 \&$ 2.3/2.4). Most of social psychology deals in more general terms with discrimination, not specifically focusing on the labor market, and mainly relying on laboratory experiments to test theoretical ideas (see sections $1.2 \& 2.3,2.4 \& 2.5$ ). More recently, also some quasi-experimental studies (audit studies, see section 2.2) have been done in economics. Still, up to now few attempts have been made to integrate economic and psychological research in this field. A short overview of the literature in both disciplines, however, indicates that the different ways of approaching the topic could supplement each other with aspects and points of view each approach alone is missing. Of course, such an integration of approaches, which differ in level of analysis, methodology and terminology, poses many problems, which will be discussed in more detail in chapter 2 . 
Before a first attempt to such an integration is made, one cannot be sure whether the gains will outweigh the problems, but, as the overview in chapter 2 shows, there is a lot of communality in the main explanatory concepts used. Differences lie rather in the focus of analysis and the specific use of the concepts, and in the research methodologies applied. Economic theories make use of 'psychological' concepts, but without considering their deeper implications, and psychologists assume that their models can be applied to all kinds of situations, including economic ones. All this said, there is reason to assume that an integrative approach can be fruitful in this area of research.

To start with, a common definition of the basic concept, discrimination, is needed. A very basic definition of (group) discrimination, which fits with economic as well as psychological approaches, is 'equals are treated unequally'. As this thesis focuses on discrimination in the labor market, the definition has to be more specific. Therefore, a definition by Blau et al. (1998, p. 186) is used: "Labor market discrimination exists when two equally qualified individuals are treated differently solely on the basis of their gender (race, age, disability, etc.)." Different treatment is only seen as discrimination, if the characteristice used for hiring or wage docisions aro not rolacod on productivity. Following a distinction made in social psychology (see section 2.1), discrimination here always refers to a behavior, i.e., just having discriminatory thoughts and ideas - stated preferences, which do not and are not expected to translate into behavior - is not defined as discrimination.

This thesis focuses mainly on labor market discrimination of women and (national) minorities with respect to hiring and pay, but generalizations to other kinds of discrimination are possible. It is assumed here that the basic underlying mechanisms are essentially the same in all kinds of discrimination, no matter, which group is discriminated against (see e.g., Hamilton \& Trolier, 1986; but see Linville \& Fisher, 1998, for counter-arguments). Discrimination outside the labor market, e.g., differential treatment of children during socialization or discrimination in housing, is, for simplicity, not considered, even if such discrimination can be the basis for later discriminatory outcomes in the labor market.

In the following, the main theoretical approaches to explain discrimination in both disciplines, which will be the basis of this integration, are sketched shortly. A more detailed description of economic and psychological theories and concepts is provided in chapter 2. The short description of economic and psychological concepts here is followed 
by a brief description of the theoretical and methodological approach this thesis adopts. At the end of the latter description, an overview of the chapters of the thesis is given.

\subsection{Economic and psychological approaches to discrimination}

Various theoretical approaches have been developed in economics to explain discrimination in the labor market, most of which are based on the neoclassical choice model. A prominent example is the theory of discriminatory tastes of Becker (1957, 1971). This theory conceptualizes discrimination as motivated by a given personal prejudice, or discriminatory taste, against associating with a particular group. When employers have such tastes, they are willing to pay a higher wage to the preferred workers than they would have to pay to workers of the other group. An interesting implication is that when the product market in which the employers operate becomes more competitive, discriminating employers are driven out of the market because of their inefficient behavior. Before that happens, an income effect of falling profits (Comanor, 1973) induces discriminating employers to diminish their discriminatory behavior.

Another important economic theory on discrimination is the theory of statistical discrimination (Arrow, 1973, Phelps, 1972). Statistical discrimination models argue that employers have to make hiring decisions under imperfect information. If certain groups, e.g., men and women, differ in average productivity, in the variance of productivities, or in the accuracy of measurement of productivity - or are perceived as differing in these variables - employers use this group information to make individual hiring decisions. The resulting discrimination is individual discrimination, not group discrimination, if the productivity differences between the groups are real. If the differences are just perceived, it constitutes also group discrimination. The latter should disappear with strengthened competition, as it leads to inefficient hiring decisions, and employers as well as employees will try to get and provide more reliable information.

Social-psychological research in the framework of social identity theory (SIT, e.g., Mullin \& Hogg, 1998) suggests that strengthening competition may have quite the opposite effect on discriminatory behavior of employers. It can enhance self-relevant subjective uncertainty ${ }^{1}$ for employers, and hence induce them to identify more strongly with a salient group to which they themselves belong (e.g., men, whites), because,

\footnotetext{
' Self-relevant (subjective) uncertainty means subjectively felt uncertainty about important things in the life of a person, especially important things for self-definition, for example having a job or being able to make a living, or being successful in some important task. See also section 2.6 .2 for an elaboration.
} 
following SIT, this reduces the (aversive) feelings of uncertainty. In its turn, this will prompt them to rely more on stereotypes and prejudices in their hiring decisions, and hence reinforce their discriminatory behavior. Even employers who initially do not have a discriminatory taste (the existence of whom is often assumed in the context of Becker's theory) may, by this psychological mechanism, develop a discriminatory taste when competition intensifies. ${ }^{2}$ In such a situation, strengthening competition will not drive all discriminating employers out of the product market in the long run. Following socialpsychological literature, the strength of prejudice itself and the extent to which it translates into behavior ${ }^{3}$ can be influenced by competition.

The different predictions from both disciplines with respect to the effects of competition, which become visible here, are one more reason to assume that an integration can provide interesting new insights about discrimination in the labor market.

\subsection{The approach of this thesis}

The basic aim of this thesis is to find out whether an integration of psychological and economic concepts with respect to discrimination in the labor market can add to current knowledge and contribute to a better understanding of the phenomenon. The integration refers also to the methods used, as economic modeling is complemented here by experiments and quasi-experiments, which borrow from psychological as well as economic experimental methods.

For this integration, some of the standard variables of economic explanations of discrimination will be treated differently than in most standard economic models. First, competition is seen as an activity, i.e., the analysis is more explicitly focused on the behavior of people involved in competition than in standard analyses. Economics hardly looks at competition as an activity of people (see, e.g., Blaug 2001; Zafirofsky, 2002). Moreover, when economics investigates the 'process' of competition (see, e.g., Schinkel, 2001), it usually takes preferences as given. Social psychology and some economic approaches (see e.g. Arrow, 1973; Coate and Loury, 1993; Farmer \& Terrell, 1996), however, assume, that the process or the activity of 'competing' might influence those subject to it not only in an 'economic', but also in a 'psychological' way.

\footnotetext{
${ }^{2}$ Such employers may initially already have an (unconscious) prejudice, which, in our approach, is distinguished from having a discriminatory taste. Only when group identification is sufficiently strong, the prejudice will lead to a (stated or revealed) discriminatory taste (see chapter 3 for details).

Social psychologists controversially discuss in how far 'attitudes' (i.e., evaluative judgements, see footnote 12) translate into behavior (see, e.g. Eagly \& Chaiken, 1998).
} 
Therefore, it is secondly assumed here that during the strengthening of competition, cognitive and emotional factors and the interaction of such factors can change and can influence (discriminatory) preferences (and hence behavior). This implies that variables, which are usually treated as exogenous by economists, are assumed to be endogeneous. The activity of competition could for example, from the point of view of psychological theories, lead to changes in preferences, e.g. in discriminatory tastes: For example, in the social-identity approach - as opposed to Becker's theory - 'discriminatory tastes' are not exogenously given and constant, but are influenced by environmental variables like competition. This works via identification with a social group to which employers belong (cf. Akerlof and Kranton, 2000). Analyzing how the activity of competition influences cognitions, emotions, and hence behavior can lead to deeper insights in what determines discrimination in the labor market. What specific long-run equilibrium is reached in such a case is not clear from the outset, and is not the focus of interest here.

The general aims of this thesis therefore are: (i) To make a comparison of the relevant literature in economics and social psychology, (ii) to develop an integrated, testable theoretical model making use of both literatures, (iii) to test at least part of the predictions of this model experimentally and (iv) to find out, whether very competitive market settings allow at all for psychological factors to work.

The basic questions the thesis wants to answer therefore are whether an integration of economic and psychological factors can explain discriminatory behavior in the labor market better than one approach alone; whether an integrated theoretical model can lead to new testable predictions; and finally, whether experimental tests can provide evidence for at least some of these predictions. The structure of the thesis broadly reflects the general aims:

Chapter 2 first describes empirical evidence of discrimination in the labor market. Next, it gives an overview of the most prominent social-psychological and economic theories dealing with discrimination (on the labor market). The chapter compares definitions of the key concepts in both disciplines and shows how they can be integrated. Integrative definitions of these key concepts are developed. It further motivates the integration, discusses the methodology, and shows which problems arise. In chapter 3 an integrated utility model is developed. It is described intuitively first, and then developed formally. Predictions for strengthening competition are derived, and it is discussed in how far the integrated model contributes to an understanding of discrimination in the 


\section{Introduction}

labor market. Chapter 4 describes a series of experiments, which partially build on the theoretical model developed in chapter 3 . The experiments test some of the predictions of the model in a controlled laboratory setting. Results are discussed in the light of the theoretical model. In Chapter 5, a quasi-experimental study, which also tests part of the predictions of the integrated model, is described. It has been conducted using a businesssimulation game and looks at nationality-based discrimination. Chapter 6 describes an experiment done to look at wage differences in experimental markets more in general. It tests, whether efficiency-wage effects could be an explanation for the gender wage gap, and whether in very competitive market settings other than strictly economic forces can influence behavior. The idea behind this approach is explained and results of the experiment are described and discussed. Chapter 7 summarizes and jointly discusses the implications of the theoretical, experimental and quasi-experimental research. 


\section{The integrated framework \\ - main factors influencing discrimination, empirical evidence, and methodology}

\subsection{Introduction}

This chapter gives an overview of the economic and social-psychological research of relevance for an analysis of discrimination in the labor market. It is not meant though as an exhaustive review of both literatures, but rather as an introduction in either field for those of the readers having a background in the other discipline.

Many concepts used to explain discrimination on first sight seem common to both disciplines, but they are often defined differently in each of them. To be able to integrate the approaches, it is necessary to be aware of these differences and to make a clear choice of the definitions used for the integration. Doing this, new problems arise. Economics is, in general, using well-operationalized and formalized concepts, which are part of a general, comprehensive theoretical framework. Social psychology, on the contrary, mainly looks at many different detailed aspects of (individual and group) behavior, missing a more general, unified theoretical framework. The concepts used are not always explicitly and clearly defined. There are sometimes even differences in definitions between research schools and traditions, which are not always made explicit. This makes it difficult to choose the definition of a concept to rely on, and to be precise on the consequences of this choice. Brewer (1994) gives a good example of the difficulties one encounters when trying to summarize social-psychological research in the field of discrimination and related concepts: „Understanding prejudice and intergroup conflict invokes virtually every area of social psychological inquiry, including the study of person perception, social attitudes, aggression, self-esteem, social comparison, equity, cooperation and competition, and conformity and compliance. Further, the study of prejudice crosses all levels of analysis, from intra-individual to interpersonal to intergroup processes. [...] unfortunately, much of this knowledge is encapsulated in isolated - sometimes competing - research traditions. The lack of integration of social psychological research and theory relevant to the understanding of prejudice and discrimination makes it difficult to tell the story to the world" (p. 316f.).

As an attempt to overcome problems resulting from that situation, this chapter compares definitions of the main concepts in social psychology and economics and 
explains the choice of definitions for the integrated model. Further, benefits, challenges and problems of the integration are discussed, also focusing on methodological questions.

The selection of the research reported here followed the principle to report only what is necessary for an understanding of the motivation for the integration in general and of the integrated model and the experimental research conducted in particular. The review tries to organize the diverse, sometimes contradictory findings of both disciplines, and to place them into a larger framework.

Economic research is focusing mainly on discrimination in the labor market, whereas social-psychological research is dealing with discrimination on a more general level, seldom explicitly with the labor market. As the principles discovered generally are assumed to be basic principles of human behavior, they can, however, be applied to labor market discrimination. Most commonly, social psychological research on discrimination makes a division into three components (Fiske, 1998):

- Discrimination as the behavioral component,

- Prejudice as the emotional component,

- Stereotyping as the cognitive component.

In addition, the integration makes use of the concept of social identity, and, as environmental variables rather stemming from economic theorizing, looks at the role of uncertainty and competition.

Both disciplines deal with most of these concepts in their analyses of discrimination, but in very different ways. In the following, it will be discussed more in detail, how each discipline uses these five interrelated concepts - stereotyping, prejudice, social identity, uncertainty, and competition.

The chapter is in general organized along these concepts, but first empirical evidence of discrimination in the labor market is reported. The next section then reviews research on stereotypes in social psychology and related economic research. Section 2.4 deals in the same way with prejudice. The following section treats social identity, mainly in a social-psychological framework, as not much economic research considers identity. Section 2.6 sketches the role of uncertainty as viewed in both disciplines. Section 2.7 describes the role of competition from an economic and a social-psychological point of view. Section 2.8 finally discusses methodological differences between the disciplines and sketches the integrated framework. 


\subsection{Empirical evidence for discrimination in the labor market}

In economic research, a large amount of econometric literature explores the gender (and racial) wage gap (see e.g. Stanley \& Jarell, 1998, for a discussion of this approach), and measures which part of it can be explained by human-capital factors and other productivity- and wage-related variables, e.g. specific preferences, employment in specific sectors and rewards for skills, overall wage structure, wage inequality in a society, etc. The unexplained residual is usually interpreted as evidence for discrimination. Different decomposition techniques based on different reference populations are used. In the framework of the most widely used decomposition technique, the Oaxaca/Blinder decomposition, it is usually assumed that the returns to job characteristics of women in the absence of discrimination would be equal to those of men (see, e.g., Oaxaca, 1973; Blinder, 1973; Oaxaca \& Ransom, 1994).

All studies find a wage gap between blacks and whites and between women and men, and all studies also find an unexplained residual. Some of the studies use longitudinal data (e.g., Shenhav, 1992; Blau \& Kahn, 1994; Datta Gupta et al., 1999) and analyze the development of discrimination over time. Most of the research on wage discrimination has been done in the U.S., research on gender discrimination as well as on discrimination of blacks.

Studies dealing with the gender wage gap report a trend towards narrowing of the gap during the last 40 years (e.g., O'Neill, 1985; Fuchs, 1986; Ashraf, 1996; Blau \& Kahn, 1994; 1997; 2000; 2002). Overall, the female-to-male wage ratio in the US, estimated on a yearly basis, increased from $59 \%$ in 1981 to $72 \%$ in 1998 . Using weekly earnings of full-time workers, it increased from $61 \%$ to $77 \%$ between 1978 and 1999 (Blau et al., 2002). Studies dealing with European countries find similar patterns as in the U.S., with a somewhat better general situation in Scandinavian countries and a somewhat worse situation in Germany (e.g., Mavromaras \& Rudolph, 1999), and stagnation in the development of the male-female wage gap in the 1990s (e.g., Blau \& Kahn, 1994; Datta Gupta et al., 1999). One of the most important questions with respect to an explanation of the narrowing of the wage gap is whether this is only due to changes in 'hard', objective (e.g., human-capital) variables, or whether there is also a change in the amount of discrimination ${ }^{4}$. Evidence on this question is mixed. There is general consensus

\footnotetext{
${ }^{4}$ For an analysis of the role of human-capital variables see e.g. Anderson \& Shapiro (1996), for other variables see e.g. Fortin \& Lemieux (1988).
} 
nowadays that advancement in the acquisition of human capital by women (blacks) he played a significant role in closing the wage gap. Debate continues over the relationshi? of those human-capital factors with discrimination, and the relative importance of certai of these factors (see, e.g., Sandell \& Shapiro, 1978; Corcoran \& Duncan, 1979; O'Neil, 1985; Blau \& Ferber, 1992; for a review of part of this discussion see Blau et al., 1998 2002). Blau \& Kahn (2000) explain part of the narrowing of the wage gap with the fad that new cohorts entering the labor market now have better chances. A rise in wages fo older women plays a role as well. In general, estimates of the unexplained part and if development vary widely, influenced by which variables are included in the equation' (see, e.g., Blau et al., 1998). Estimates range from up to two thirds of the wage gap t/ around $10 \%$ of it (see, e.g., Blau \& Kahn, 2000). Most studies report a decline in the unexplained part alongside with the decline in the wage gap, but Blau \& Kahn (1994) point to the fact that this does not necessarily result from a decline in discrimination, bu can also, e.g., result from an "upgrading in women's unmeasured labor market skills" (p 31). A recent study on top corporate jobs (Bertrand \& Hallock, 2000) focusing on the five highest paid executives of U.S. firms between 1992 and 1997 and controlling for age, experience, and company-related factors, finds that the part of the $45 \%$ wage gap between women and men in these positions, which remains unexplained, is less than $5 \%$

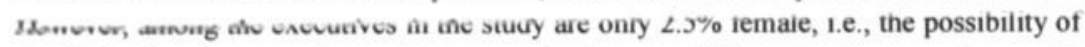
discrimination in promotion or by segregation is not ruled out by these results - as in most studies of this kind.

Research on wage discrimination against blacks in the U.S. also reports a decline in the wage gap between black and white men (e.g., Blau \& Beller, 1988). The situation for black women is somewhat more complex (see e.g., Shenhav, 1992; Ashraf, 1996). Blau et al. (2002) show that in 1999 the female-to-male income ratio was higher among blacks than whites ( $83 \%$ as compared to $71 \%$ ). The median income of black males in the same year was $77 \%$ of white males', but the income ratio for black women was $90 \%$ compared to white women. Again, estimates of the unexplained part of the gap vary widely.

The main problem with the econometric approach is that the estimation of the non-explained part of the wage gap varies widely depending on the specific models and decomposition methods the studies use. Specification and omission of variables and measurement of wages and other important variables strongly influence the results. 
Furthermore, selection bias' has an impact on the results of such studies. Passage of time and gender of the researchers (male researchers report a larger wage gap) also have been found to influence estimates of the wage gap (see the meta-regression analysis by Stanley \& Jarell, 1998). Therefore, some researchers took different approaches to find out whether discrimination against certain groups exists.

One alternative approach is "correspondence testing" (also known as "auditing"), which is focused at discrimination in hiring. Matched pairs of written job applications, varying only in race or gender of the applicants, are sent in response to advertised vacancies, to test for discrimination in hiring at the initial stage of selection for a job interview. In some studies actors were sent to job interviews. Statistically significant amounts of discrimination were found in several countries, against women as well as against people of certain racial origin, at least for some of the jobs tested. Examples are Jowell \& Prescott-Clarke (1995), Neumark \& McLennen (1995), and Goldin \& Rouse (2000). The latter use a slightly different methodology: They analyze in how far the introduction of 'blind' auditions for professional orchestras has led to more hiring of women, and find that it significantly reduced discrimination against women. A review of more audit studies can be found at Heckman \& Siegelman (1993).

Of course, this kind of studies faces problems as well. Using matched applications, the question always remains whether these applications really only differ in gender or race, and not also in other, maybe productivity-related aspects. This results from the fact that applications can be matched, but of course not made exactly equal. This becomes even more relevant for such studies where actors 'play' applicants. Furthermore, this approach can rather be used to show that discrimination in hiring exists, and does not account for wage discrimination, as the 'applicants' never are really hired.

Relatively few empirical studies explicitly aim at testing the common economic theories, i.e., the 'taste' model (Becker, 1957) and statistical discrimination models (Arrow, 1973, Phelps, 1972). The methodology used in the studies that do so is usually some kind of regression-analytical technique, using field data.

Evidence on Becker's model is mixed: Some studies report supporting evidence, such as those of Haessel \& Palmer (1978), Fujii \& Trapani (1978), Luksetich (1979), Ashenfelter and Hannan (1986), Peoples \& Saunders (1993), Heywood \& Peoples

\footnotetext{
${ }^{5}$ Selection bias refers to the problem that people taking part in such a study are not a random selection of the population of, e.g., women.
} 
(1994), Hellerstein et al. (1997), and Bruggert \& Schippers, (1999). A coupl of other studies, e.g. Shepherd \& Levin (1973), Oster (1975), and Baldwin \& Johnson 1996) do not find much support for it. An example of recent interesting research testing te Becker model is a study, which has been conducted by Szymanski (2000) and Feston \& Szymanski (2000). They test for employer and customer discrimination by ree in the English professional soccer leagues. They find evidence that clubs in fact disiminate, and also incur 'productivity' losses from this behavior. The reason for discrimation in this case is employers' preferences, not customers'. Furthermore, it was evient that discrimination is not based on wrong estimates of the productivity of black pyers, as performance criteria in soccer are quite clear. However, the labor market for priessional soccer players has been organized competitively only recently, which makes th authors think that conclusions with respect to the predictions of the Becker model ar not yet possible.

Many empirical studies deal with the question whether higher turnoverates can be seen as a reason for statistical discrimination against women (see e.g. Bh et al., 1998, Altonji \& Blank, 1999). Empirical evidence with respect to turnover or qu rates is mixed. Quit rates are said to be higher overall for women than for men, ut these differences disappear when characteristics of workers, of the labor market, ad of the spectrie jobs̀ are controllëd tô (Vì́cusi, 1980; Blau \& Kahn, 1981; Osterman, 1982; Haber, Lamas \& Green, 1983; Light \& Ureta, 1992; Sicherman, 1996). An example of an empirical analysis of statistical discrimination is a study conducted by Altonij and Pierret (1997), who test for statistical discrimination by firms in a situation where firms can learn. Using data of young men from the NLSY (National Longitudinal Survey of Youth), they find evidence that firms statistically discriminate using education (years of schooling), but not using race. This constitutes statistical discrimination in the sense that years of schooling is a characteristic which is not perfectly related to productivity and which is used instead of collecting individual (test) information.

This very restricted overview of empirical evidence in economics only aims at providing some evidence for the relevance of the topic, and at showing the spectrum of empirical methodologies used in economic research in this field. Social psychology in contrast nearly only relies on laboratory experiments, as will be shown below.

The following sections will now discuss the most important concepts and their definitions in both disciplines, and develop the definition(s) of each concept to be used in this thesis. 


\subsection{Stereotypes}

\subsubsection{Stereotypes in social psychology}

Among the most important social-psychological determinants of discrimination are stereotypes (e.g. Fiske, 1998). In social psychology, stereotypes are most often, on a high level of abstraction, defined as common beliefs about personal attributes and behavior of members of a group. More specifically, they are defined as the subjectively expected correlation between characteristics of individuals and membership of a certain group (translated from Fiedler, 1996b p. 162). Still, a clear-cut, universally used definition of stereotypes in social psychology is hard to find. For example, stereotypes can be seen either as consensual beliefs, or as unjustified beliefs. Gardner (1994) goes so far as to state that „[...] the terms prejudice and stereotypes are bad in [...] their scientific utility: Both terms have acquired such a great deal of excess meaning (often in the absence of any empirical justification) that when researchers gather to discuss them, they often talk at cross purposes. Theorists use the same terms to refer to very different phenomena, often referring to the same previous research, and then are surprised to find that they disagree on very basic conclusions." (p. 1)

The basis of stereotyping is the differential perception of groups. For an effective categorization, easily identifiable features of the perceived objects are used, e.g., race, gender, or age. The features used for categorization do not have to be of any social importance, or to be indicative for something else than the membership in a certain group. Research in the framework of the minimal-group paradigm has shown that many of the effects of stereotyping can be evoked just by artificially constructing two groups by means of, e.g., preferences for a painter (Tajfel et al., 1971). One assumption which has important (methodological) implications, but which is subject of disagreement among social psychologists, is the assumption that the fundamental nature and functioning of all stereotypes is the same (Hamilton \& Trolier, 1986; for a contrary opinion see, e.g., Linville \& Fisher, 1998).

Stereotypes have their origins in the socialization process. Cognitive mechanisms - information-processing biases - can lead to the development of stereotypic belief systems; social-learning processes further its development. The cultural context defines the utility of various categories, and people develop consensus about such cultural categories. Stereotypes are thus at least partially linked to experiences made in reality. In his "BIAS"-model, Fiedler (2000) points to the fact that stereotypes and stereotype- 
driven behavior can be explained parsimoniously by the effect of biased samples, for example concerning sample size for in- and outgroup ${ }^{6}$ : "...ecological samples are virtually never random; some data are more likely to be sampled than others. Most real samples are therefore biased in multiple ways and subject to multiple boundary conditions (Wells \& Windschitl, 1999). Judgments that have to rely on these samples are consequently infected by the same biases. Biases occur despite, or exactly because, the judgments are often remarkably accurate - reflecting precisely the information conveyed by the sample. Crucial to this cognitive process is the lack of meta-cognitive devices that would be necessary to understand sampling constraints and to correct sample statistics accordingly." (p. 6). Therefore, many so-called 'biases' in stereotype verification and differential perception of in- and outgroups can be explained by the fact that stereotypeconfirming or ingroup samples are usually bigger, and hence the pattern of relevant attributes of the ingroup sample correlates more strongly with the ideal pattern than the pattern of relevant attributes of the outgroup sample does.'

The fact that stereotypes are related to experiences is reflected also in some findings concerning the accuracy of stereotypes. Ottati and Lee (1995) review studies on stereotype accuracy and find evidence for convergence of the stereotypes various groups hold of particular other groups and the view groups have of themselves, and of stereotypes and more 'objective' indicators. Furthermore, they find that people are sensitive to within-group variations. This is also confirmed by a review of Linville \& Fisher (1998), who show that people are able to make reasonably accurate estimates of group variability, even if they are sometimes biased (see also Judd, Ryan \& Park, 1991; Quattrone \& Jones, 1980).

Categorization of individuals into groups can have many different consequences. Only those of the known consequences which are relevant for the topic at hand are now described shortly (following an overview of Hamilton \& Trolier, 1986):

- Members of the same category are perceived as being more similar to each other, members of different categories as being more dissimilar, even when the assignment to the groups is arbitrary and the feature for the group identification is uninformative for anything else than the category (see minimal-group paradigm, e.g. Tajfel et al., 1971).

\footnotetext{
6 'Outgroup' in social psychology means any group different from one's own (which is called 'ingroup'), e.g. soccer supporters of another team than the one oneself supports, people of a different nationality or the other sex. We use 'ingroup' here for the discriminating group, and 'outgroup' for the group discriminated against, to make integration with social-psychological theories of intergroup conflict easier.

${ }^{7}$ It would lead too far to explain the model in detail here, but see Fiedler et al. (1999a, b), Fiedler (2000).
} 
- The perceiver's causal attributions about the behavior of members of the groups are influenced by the categorization.

- Categorization influences what aspects of the information at hand are attended to, how it is interpreted, and what part of it is retrieved from memory later. Stereotype consistency of the information can influence the ease of processing of information in both directions (making either more or less stereotypical information easier to process) depending on the context (see e.g. Hamilton \& Rose, 1980; Lui \& Brewer, 1983; Bodenhausen \& Wyer, 1985).

- The judgmental standards used in a situation where people have to make social judgments are influenced by stereotypes. Standards often differ from one social category to another (see e.g. Biernat et al., 1998; for an interesting experimental study see Biernat \& Kobrynowic, 1997).

- Behavior that involves the allocation of limited resources (zero-sum allocation, e.g., of money or jobs) is said to be strongly influenced by stereotypes (e.g. Biernat et al, 1998).

Research has shown that relying on stereotypes can be functional, as it helps economizing on cognitive resources in a complex world (e.g., Tetlock, 1992; Fiske, 1998). People categorize others into groups to reduce the amount of information they must contend with. The link of such a category with certain traits, i.e., a stereotype, allows the perceiver to predict the course of an interaction with a member of that category, because it makes him 'know' certain traits of the target. Relying on stereotypes therefore helps people to interact more easily in social situations. This functionality is reinforced by the phenomenon of behavioral confirmation (self-fulfilling prophecies), which is considered in economics as well. It describes a situation in which beliefs and expectations 'create their own reality' (Snyder, 1992). Merton (1948) defines the selffulfilling prophecy as being a false definition of the situation in the beginning, which then evokes a certain behavior, making the originally false conception come true. In other words, the perceiver behaves according to his stereotypes and thereby elicits confirmatory stereotypical behavior from the target. This makes the behavior of the target more predictable and thereby more controllable (see e. g., Darley \& Fazio, 1980; Snyder, 1984; for a review of the literature see Snyder, 1992). Claire \& Fiske (1998) extend this idea to whole societies. In their view, if powerful groups in a society share a stereotype, and the stereotype continuously influences the target persons or groups, it may influence them so far that they really fulfill the resulting expectancy. This facilitates 
social interactions, but it can have negative consequences for members of the stereotyped groups.

Some authors (e.g., Jost \& Banaji, 1994; Snyder \& Miene, 1994) argue that stereotypes have a justification function: Jost \& Banaji (1994) distinguish ego justification, group justification, and system justification. They go so far as to argue that under some circumstances, stereotypes that serve to justify an existing order can operate even against individual or collective self-interest ${ }^{8}$. Klein \& Kunda (1992) extend this argument to all kinds of reasoning: They assume that people usually want to justify their conclusions and beliefs rationally. Further, people are said to attempt to rely as well as possible on rules and evidence given. To cope with biases in cognitions which arise despite of the strive for accuracy these biases are extended to general belief systems to make them seemingly consistent. Motivational effects like this are especially likely when the knowledge base is ambiguous or self-contradictory, which is often the case with beliefs about people and society. As Damasio (1994) formulates: "The personal and immediate social domain is the one closest to our destiny and the one which involves the greatest uncertainty and complexity" (p. 169). Furthermore, stereotypes can be 'used' to justify prejudice, i.e., negative emotions towards certain groups (see section 2.4.1).

It thus has been shown that stereotypes can be used to simplify complex social situations. However, they do not necessarily influence behavior (see e.g. Linville \& Fisher, 1998), and they can influence behavior context-specific. A variety of factors may influence the use of stereotypes (and the emergence of prejudice and discrimination) in interactions with members of stereotyped groups. Among the factors which give stereotypes an advantage compared to more evidence-based processing are, e.g., mental busyness, information overload, time pressure, pressure to implement a decision, and certain emotional states (moods), including happiness, anger, and anxiety (e.g., Isen et al, 1985; 1987; Pratto \& Bargh, 1991; Esses, Haddock \& Zanna, 1994; Dijker \& Koomen, 1996). It could be shown that the actual presence of information may not be crucial for the question whether people use stereotypes. The feeling of being informed, even without any information objectively present, may be sufficient to allow for stereotypic judgment (Yzerbyt et al., 1994). What probably matters is people's own impression of the quality of the information at hand, which is based on personal experience and one's own group

\footnotetext{
This is an important aspect with respect to labor-market discrimination, because it implies that under some circumstances, stereotypes justifying an existing order can operate even against (financial-) economic rationality.
} 
membership (Fiske, 1998). Bodenhausen et al. (2001) argue that stereotypic beliefs can just be 'added' to the information available. If the basic information is ambiguous, the 'added' stereotypical information can have a strong influence.

Another important question is, which of the possible stereotypes will be activated. In every social interaction, several stereotypes could possibly be activated, e.g., in a hiring situation, an Asian women could be first and foremost seen as an instance of the category 'Asians' or of the category 'women'. Bodenhausen et al. (1998) assume that there is a 'cognitive competition' among the various possible categorizations, and which one wins is affected, among other factors, by the recency of category activation, the presence of situational cues, and contextual salience.

A lot of research discusses how stereotype change can be reached. Obviously, the first condition necessary for stereotype change is awareness of the possibility that stereotypes influence a perceiver. This is not given in the case of implicit stereotyping effects, i.e., when stereotypes work unconsciously ${ }^{9}$ (see, e.g., Greenwald \& Banaji, 1995).

Awareness alone is not sufficient to change stereotypes or inhibit the use of them, because people may deceive themselves about their susceptibility to the influence of stereotypes (Bodenhausen et al, 1998; see also Gaertner \& Dovidio, 1986). Therefore, motivation to control for the effects of stereotypes (and prejudices) is necessary as well. Furthermore, certain attentional requirements have to be fulfilled. Trying to avoid a particular thought actually may result in its hyper-accessibility. This may, in case of too few resources for the operating process, result in more biased construals. This 'rebound' effect was found to be not limited to impressions and judgments, but also to find behavioral expression (Macrae et al., 1994). Bodenhausen et al. (2001) add as a further condition for stereotype change that the individuating information must be unambiguously in poor fit with the stereotypic expectations.

Further, the level of homogeneity of the outgroup is important. Linville \& Fisher (1998) report that when the outgroup is in general very homogeneous, a counterstereotypic new exemplar is unlikely to be classified as an instance of the group, and therefore will not lead to a change in the stereotype. In a very variable group, on the other hand, the inclusion of an(other) atypical member has little effect on the overall group stereotype. Thus, counter-stereotypic information has to be presented in the

\footnotetext{
As using stereotypes preserves mental resources, this relative automaticity is also functional.
} 
context of a typical outgroup member, because only then, generalization from specific individuals to the group as a whole is possible (Hewstone \& Lord, 1998). If stereotypedisconfirming information is presented in combination with an otherwise atypical member of the group, this will rather lead to the formation of a 'subtype' (e.g., career women as a subtype of women; see Fiske, 1998). Using subtypes is socially and cognitively pragmatic in that the overall stereotype can be maintained and the status quo can be justified and perpetuated.

Important for stereotype change is also whether people themselves can control the nature and amount of information they receive about a target group, or whether they are forced to process all the information available (Johnston \& Macrae 1994). People usually show a preference for stereotype-matching information and therefore do not change their stereotypic impression of the group when they can choose the information processed. On the other hand, if they are forced to process all information, their stereotypic evaluation of the group can diminish. This implies that in naturalistic, information-seeking settings, the resistance of stereotypes to change is high.

If one wants to achieve stereotype change in a situation of group contact, as in the 'classical' interventions (Allport, 1954; Cook, 1962; Amir, 1969), membership of the groups must be obvious to make generalized attitude change possible (Hewstone \& Lord, 1998). The situation thus must be clearly an intergroup situation, not an interpersonal situation. However, even then it is possible that only the perception of the specific outgroup changes: Hewstone \& Lord (1998) find that stereotype change achieved in one contact situation usually does not generalize, neither across situations nor across persons. Fiske \& Stevens (1993) add as another condition for stereotype change through contact that the two groups must have equal status.

Another problem with achieving stereotype change by providing any kind of stereotype-inconsistent information is that such information tends to be experienced as threatening and thereby can create negative affect - which in turn can negatively influence behavior towards the outgroup (Bodenhausen et al., 2001). Jussim et al. (1995) see the partial independence of affect and cognition as a major obstacle for change, because affectively based discrimination could continue after the change of the stereotype.

This overview of research on stereotypes in (social-) psychology gives an impression of the huge amount of studies done in this field. In economics naturally far less research is concerned with stereotype-like phenomena. 


\subsubsection{Statistical discrimination - stereotypes in economics}

Economic theory dealing with discrimination is not usually talking about stereotypes; however, statistical-discrimination theory (Arrow, 1973, Phelps, 1972) makes use of a very similar concept. Statistical discrimination occurs when employers (correctly) ${ }^{10}$ expect that, on average, members of a certain group are less productive or less stable employees than members of another group. Since employers have to make personnel decisions under incomplete information and uncertainty ${ }^{11}$ about the productivity of an individual applicant or employee, they will, at least partially, base their decisions on their beliefs about group averages. Any information seen as correlated with productivity in the widest sense, which can easily be obtained, e.g. information about sex or race, can be used for making personnel decisions. (Perceived) group differences can exist not only in average levels of productivity or variance of individual productivity, but also in the reliability or accuracy of the way the productivity of individual members of the groups is measured (see e.g. Aigner \& Cain; 1977, Lundberg \& Startz 1983; Lundberg 1991). When differences between groups are real, the result is discrimination against individual (out-)group members in hiring, pay or promotion. In this case, statistical discrimination cannot be regarded as discrimination against groups, and is rational from a profit-maximizing point of view.

The perception of a productivity difference may also work as a self-fulfilling prophecy by the adverse incentive effects of lower wages, less productive jobs and fewer training facilities for outgroup members (e.g., Arrow, 1973; Coate and Loury, 1993; Farmer \& Terrell, 1996). Berk (1999) relies on a similar mechanism to reach the opposite conclusion: Self-selection bias resulting from discrimination should reduce discrimination in the long run. From a discriminated group only those with very high qualifications will apply for certain jobs because the less qualified cannot expect to have a chance. This should then lead to the impression that the discriminated group is on average more productive than the other group. Neal \& Johnson (1996) report empirical evidence for such an effect. This is a mechanism, which resembles the basic idea of the BLAS-mechanism Fiedler proposes (e.g., Fiedler et al., 1999a, b).

\footnotetext{
${ }^{10}$ Aigner \& Cain (1977) argue that statistical discrimination can only persist when group differences are real, and not just perceived by the employers. Blau et al. (2002) include (wrongly) perceived differences into their description of statistical discrimination. In the following the latter will be referred to as weak variant of statistical discrimination.

"See section 2.6 about uncertainty and imperfect information.
} 
When feedback effects or self-fulfilling prophecies do not apply, and there continues to be no actual difference in average productivity between the ingroup and the outgroup, a false perception may be expected to yield to new information about the absence of an actual productivity difference over time. If, however, the perception originates from a personal prejudice, it may serve as a rationalization and justification for this prejudice and the ensuing discriminatory behavior. This can be explained from the psychological inclination of a person to reduce cognitive dissonance between, on the one hand, her perception and, on the other hand, her discriminatory prejudice (and behavior) (Festinger, 1957; Arrow, 1973).

\subsubsection{Summary and definition used here}

To summarize, social-psychological research basically defines stereotypes as common beliefs about personal attributes and behavior of members of a group. The basis of stereotyping is the differential perception of groups based on easily identifiable features like race or gender. Stereotypes are achieved during socialization, and to a certain extent reflect real group differences. The categorization of individuals into groups can have many different consequences, which often, but not always, have aversive consequences for the stereotyped groups. Relying on stereotypes can be functional in that it helps economizing on cognitive resources and facilitates social interactions. Stereotypes can also be used to justify prejudices. However, they do not necessarily influence behavior in all contexts. Whether people behave following their stereotypes depends, among other factors, on personal experience and one's own group membership. In general, stereotypes are highly resistant to change, especially in naturalistic, information-seeking settings.

In economics, the explanation of discrimination advanced by statisticaldiscrimination theory corresponds to the basic concept of a stereotype, namely a (correct or wrong) perception of a group average, which is then applied to judge members of that group. Employers have to make personnel decisions under incomplete information and therefore partially base their decisions on their expectations about group averages. Any easily obtainable information seen as correlated with productivity or job stability, e.g. information about sex or race, can be used for making personnel decisions. The perception of differences between groups can be correct or false. These two cases have different implications for discriminatory behavior in the long run. 
The definition of stereotypes my research is based on is taken from social psychology: Stereotyping is "...the unconscious or conscious application of (accurate or inaccurate) knowledge of a group in judging a member of the group" (Banaji \& Greemwald 1994, p. 54).

\subsection{Prejudice}

\subsection{Prejudice in social psychology}

Recently, research in social and economic psychology has generally turned more towards analyzing the importance of emotional aspects for decision-making. Jussim et al. (1995) criticize the cognitive approach, because most judgments in their view are not purely cognitive. Even in hiring decisions, applicants are evaluated and thus, the judgment is not purely cognitive (see also Nelson, Biernat, \& Manis, 1990; Biemat, Manis, \& Nelson, 1991; Jussim, 1991). Loewenstein et al. (2001) summarize an approach, which sees emotions, more precisely anticipatory emotions, as a basic necessity for decision-making. An example is Damasio's 'somatic marker hypothesis' that posits that decision-making is guided by somatic reactions ('gut feelings') to decision alternatives that provide information about the valuation of the alternatives, e.g., whether they are risky or not (Damasio, 1994; for a related argument see also Clore, Schwarz, \& Conway, 1994).

The emotional aspect of discriminatory behavior is often referred to as 'prejudice' (e.g. Schaller et al., 1998), and this is how the term is going to be used here as well. Prejudices can be defined as negative, 'affect laden' (Pettigrew, 1997) attitudes ${ }^{12}$ towards all or most members of a certain group. Unfortunately, for the definition of prejudice in social psychology holds the same as for stereotypes - there are many variants and it is not always made explicit which variant is used.

Prejudice has been shown to be usually a better predictor for discriminatory behavior than stereotypes are (e.g. Stangor et al., 1991; Dovidio et al., 1996). Betsch et al. (2001) show that implicitly formed attitudes (value judgments) toward a target can

\footnotetext{
${ }^{12}$ The definition of attitudes also is subject of discussion in social psychology. Most generally, they can be defined as a "psychological tendency that is expressed by evaluating a particular entity with some degree of favor or disfavor" (Eagly \& Chaiken, 1998, p. 269). There is, however, some controversy whether attitudes comprise cognitive, emotional and behavioral aspects or only affective aspects (e.g. Petty \& Cacioppo, 1981). In the following, if not otherwise indicated, the one-dimensional interpretation (only consisting of affective reactions) is used.
} 
very accurately follow from value-loaded experiences with the stimulus, eve if the informational content of these experiences cannot be recalled cognitively any mos.

This does not imply that all negative prejudice against certain groups is bsed on personal negative experiences with those groups. Prejudice can also follo from 'second-hand' experiences, i.e., it can be learned from what others tell about th groups in question.

Bodenhausen et al. (2001, p. 319f.) distinguish between "chronic integrahffect", "episodic integral affect" and "incidental affect" towards certain groups. If most relevance for the research here are the first two kinds of affect. Chronic integraliffect is the enduring affective reaction to, e.g., a certain social group, which can ifluence behavior towards this group. Episodic integral affect comprises affective ractions created spontaneously in an intergroup situation. These episodic reactions can e quite different from the chronic ones. An example would be a positive experience vith one member of a generally negatively stereotyped and evaluated group. Bodenhasen \& Moreno (2000) predict under what circumstances chronic integral affect inluences behavior towards stereotyped group members: "... when perceivers (a) are unaure that they are being influenced by their chronic background feelings about the group (b) are unmotivated to correct such biases [...]; (c) lack the attentional resources that are necessary to suppress or correct for affective biases; or (d) convince themselves that their negative feelings are due to something other than the group's identity per se" (cited from Bodenhausen et al. 2001, p. 322). This is similar to the conditions for the influence of stereotypes on behavior.

Emotional reactions to members of certain groups, are probably also situationspecific: Seeing a good-looking woman in a bar will for most people not lead to negative emotional reactions, while seeing a good-looking woman applying for a top-management position might evoke such negative reactions.

Emotional factors can thus explain why e.g. a woman can be more negatively judged when applying for a certain job than a man, even if the 'cognitive' information about both is equal and the perceiver tries to avoid stereotyping. Emotions can especially become important in decision-making when no clear cognitive judgment is achieved. In such a case the existence of a (strong) emotional 'judgment' might determine the decision. 


\subsubsection{Tastes for discrimination - prejudice in economics ${ }^{13}$}

Gary Becker $(1957 ; 1971)$ conceptualized discrimination as motivated by a personal prejudice, or what he called a 'taste', against associating with a particular group. This 'taste for discrimination' is influenced in his view, among other factors, by the degree of contact between the groups in question: Becker assumes that, depending on the size of the groups, the economic significance of the groups, and the duration and level of contact between them, one group develops a preference against contact with the other group, which can vary in strength. If there is absolutely no contact or very intensive contact between the groups, no such preferences will develop. Employers, employees, and/or customers all may have such discriminatory tastes. At the same time, the model assumes that there are no actual differences in the distributions of productivities between the particular group and other groups. According to the definition of Becker, "if an individual has a "taste for discrimination", he must act as if he were willing to pay something, either directly or in the form of a reduced income, to be associated with some persons instead of others" (1971, p. 14).

The amount of money, which he ${ }^{14}$ is willing to pay, is represented by the discrimination coefficient. The discrimination coefficient can be said to represent the nonpecuniary psychological cost of employing, working with, or purchasing from a member of the particular group in terms of money. In the case of employer discrimination, the full costs of employing a member of the particular group (the outgroup) are his/her wage plus the discrimination coefficient. This means that discriminating employers will hire members of the outgroup only at a wage lower than that of members of the ingroup. Hence, in a competitive labor market, where ingroup members are paid in accordance with their productivity, outgroup members will be hired only if they are paid less than their productivity. The actual wage difference in a competitive labor market, i.e. the market discrimination coefficient, depends on the size and distribution of the individual discrimination coefficients among employers and on the relative labor supply of ingroup and outgroup members. For example, suppose there is only one industry and a fixed supply of ingroup and outgroup members such that the supply of ingroup members is one-third that of outgroup members. Further assume that ingroup and outgroup members are perfect substitutes in production and that in

\footnotetext{
${ }^{13}$ A more formal description of this model is given in section 3.4.1.

${ }^{14}$ Throughout this thesis, the male form is used to indicate both sexes. This has been done for convenience of the reader.
} 
equilibrium the labor demand by each firm is the same. The equilibrim market discrimination coefficient would then equal the first-quartile discrimination cofficient in the distribution of individual discrimination coefficients (see Becker, 1971, I 43, for a proof).

In the view of Becker, a discriminatory taste incorporates both preadice and ignorance about the absence of an actual average productivity difference. hese two concepts are sharply distinguished since "ignorance may be quickly eliminied by the spread of knowledge, while a prejudice (i.e., preference) is relatively indeendent of knowledge" (Becker, 1971, p. 16). This would imply, psychologically spealng, that a 'taste' consists of a prejudice part and a stereotype part. However, econom research building on Becker's model usually uses 'taste' as synonymous for 'prejudice'

The present analysis focuses on employer and employee discrimiation, but discrimination by customers or the government can be analyzed similarly.Employee discrimination is a situation where employees of a certain group have taste for discrimination against an outgroup. If an employee has a taste for discriminabn against an outgroup, he will demand a compensating premium equal to his discimination coefficient for working with a member of this outgroup. ${ }^{15}$ One response of his profitmaximizing employer to such a situation could be to hire a segregated work force. If this is not profitable because of substantial costs of adjustment from the previous situation, and many outgroup members seek employment in firms with discriminating ingroup employees, the market wage for outgroup members will be lower than for ingroup members (Arrow, 1973). Moreover, employee discrimination may adversely affect the morale and productivity of discriminating ingroup members who are forced to work with outgroup members (Bergmann and Darity, 1981). In addition, the productivity of the outgroup members themselves may also be reduced, as ingroup members are reluctant to give advice and assistance to new outgroup members and to include them in their mentor-protégé relationships and informal networks.

\subsubsection{Summary and definition used here}

For the psychological definition of prejudice holds the same as for stereotypes: It has developed a lot of diverging excess meaning and comprises many variants. The most basic definition sees it as the emotional aspect of discrimination (Fiske 1998). Social psychology assumes that most judgments are not purely cognitive. Recently, emotions by

\footnotetext{
${ }^{15}$ This description of employee discrimination follows the account by Blau et al. (1998, pp. 203-205).
} 
some researchers are even seen as a basic necessity for decision-making. Damasio (1994), for example, assumes that emotions always have some kind of influence on our decisions. Prejudice has been shown to be a better predictor for discriminatory behavior than stereotypes are. However, prejudice as well does not necessarily influence decisionmaking. The conditions for an influence of prejudice on behavior are in general similar to those for stereotypes. Emotions become especially important in decision-making when no clear cognitive judgment is achieved.

Becker sees prejudice (a 'taste') as the basic reason for discrimination against a particular group. This 'taste for discrimination' is influenced, in his view, by the degree of contact between the groups in question. Employers, employees, and/or customers all may have such discriminatory tastes, even in the absence of real productivity differences between the groups. Strength of this taste is represented in Becker's model by the discrimination coefficient (D), which is the amount of money someone is willing to pay, e.g., for not having to work together with a member of a certain group.

For the purpose of the integration proposed here, prejudice is defined - in agreement with social psychology and Becker's model - as the emotional aspect of discrimination:

Prejudices are negative, affective attitudes towards all or most members of a certain group (cf. Pettigrew, 1997).

\subsection{The role of social identity}

\subsubsection{The link between cognition, emotion, and behavior}

In social psychology, the link between stereotypes, prejudice, and discrimination, i.e., cognitive, emotional and behavioral components, is discussed controversially. In most real-world cases it is impossible to make a clear-cut distinction between cognitive, affective and behavioral aspects, because they are strongly interrelated and not easily disentangled (but, see Jussim et al., 1995). Up to now, however, most research focused on cognitive and emotional aspects. Not much research has been done on the behavioral component, and even less on the links between the three aspects.

There still is a lot of disagreement in the literature about how the three aspects are connected (see e.g. Brewer, 1994). Most researchers assume that consistency exists among stereotypes, prejudice, and discrimination (e.g., Merton, 1948; Allport 1954; Darley \& Fazio, 1980; Hamilton \& Trolier, 1986; Jussim, 1991; Dovidio, Brigham, Johnson, \& Gaertner, 1996; Bodenhausen, Macrae \& Garst 1998). Some, (e.g., Mackie \& 
Smith, 1998) oppose this opinion. Schaller et al. (1998) see reciprocal relations between cognitions about groups and behavior toward group members. Examples are behavioral confirmation effects (self-fulfilling prophecies, see section 2.3). Some authors see prejudice as determining stereotypes (e.g. Dovidio et al, 1996; Schaller \& Maass, 1989), others see prejudice and stereotypes as a result from attempts to justify discrimination, rather than serving as prerequisites to such behavior (e.g. Hoffman \& Hurst, 1990; Jost \& Banaji, 1994). Empirical evidence exists for different interpretations: There exists evidence that (cognitive) beliefs influence (emotional) evaluations and behaviors, but some studies also find that cognitive measures of beliefs are basically unrelated to measures of prejudice or discriminatory behavior ${ }^{16}$. Linville \& Fisher (1998) argue that cognitive-behavioral consistency with respect to stereotypic beliefs is more likely for groups with which the perceiver is relatively less familiar. A quite elaborated model of the transition from cognition to behavior is Fazio's (1999) MODE model, which basically proposes that attitudes can directly and rather unconsciously influence behavior towards an object, but cognitions can also first trigger a conscious decision about behavior. Dovidio et al. (1996) assume that intergroup cognitions are the forenunners of intergroup behaviors, and those cognitions are themselves influenced by ongoing affective states.

\subsubsection{Social identity theory and self-categorization theory}

A theoretical model, which makes elaborate assumptions about conditions for cognitive-affective-behavioral links, is social-identity theory (SIT; Turner et al., 1986), and its extension self-categorization theory (SCT; e.g., Haslam et al., 1996). The research reported here uses these theories as the general social psychological basis, because they integrate the social context into an analysis of cognitive (and affective) antecedents of group behavior in a way which is well-suited for an integration of economic factors. In economics, contrary to most social psychology, the social context, e.g., market institutions, is usually seen as a very important factor. In my view, the low amount of interest in how institutions shape behavior is a shortcoming of today's socialpsychological research. For an integration with economic research, a psychological theory which makes assumptions about how the (social) context influences cognitions and behavior is well-suited (c.f Friedman \& Sunder, 1994). Furthermore, as the aim of this thesis is to explain behavior using cognitive and emotional factors, a theory is

\footnotetext{
${ }^{16}$ This can in part probably be explained by social desirability influencing cognitive measures more strongly.
} 
needed which makes statements about the link between cognitions, emotions and behavior.

In short, social-identity theory postulates a link between social identification with existing groups and the tendency to rely on stereotypes and prejudice in behavior, i.e., to discriminate. Self-categorization theory elaborates on the cognitive mechanisms, which influence social identification. Researchers working in the framework of the socialidentity/self-categorization approach believe that a purely (individual) cognitive analysis of human behavior is incomplete and does not sufficiently take the social context into account (see e.g. Haslam et al., 1996). SCT therefore aims at understanding "experience and behavior as the product of an interaction between psychological variables [...] and "social reality"[...]"(Spears, 2001, p. 172). Group behavior and the distinction between ingroup and outgroup are a central point in the explanation of discriminatory behavior. Groups can be 'real' groups like work teams or sports teams, but they can also be more abstract, wider groups, like women and men or even, as some experiments with the minimal-group paradigm show (Tajfel et al., 1971), totally artificial groups like 'blue' or 'red'. Consequentially, (content and use of) stereotypes are in SCT assumed to be influenced by the social context. They can "vary with intergroup relations, the context of judgement and the perspective of the perceiver" (Turner 1999, p. 26).

SIT and SCT basically argue that people have personal and (one or more) group identities. In some situations the personal identity is more salient; in other situations a specific group identity is more salient. Self-categorization means that one perceives oneself in terms of a certain group, i.e., 'depersonalizes' to a certain extent. Hogg (2001) even states that the group is more important than individuality or interpersonal relations: "The argument rests on the ubiquity of groups in our lives, the need to represent a complex social world in terms of prototypes representing different groups, the fact that we are born into groups and categories [...]." (Hogg, 2001, p. 138f.).

People identify with groups they belong to, to enhance their self-esteem and to reduce uncertainty in their life (Mullin \& Hogg, 1998). The social context of the group is very important in determining which group one identifies with, how strongly one identifies, and how the group influences social identity and behavior (e.g., Ellemers, Spears \& Doosje, 1999). Uncertainty, e.g. by some threat, evokes self-categorization. Following Spears (2001, p. 190), it is important to notice that only group-level threats have been found to elicit group-level reactions. Many threats, however, can be perceived either as a group-level threat or as an individual-level threat, depending on the situation. 
A perhaps surprising idea is that one possible source of uncertainty evoked by a group threat is intergroup contact, because it can challenge beliefs about the groups and their relationship (Hogg \& Mullin, 1999).

If, in a situation of uncertainty, identification with an ingroup is possible, a subjective feeling of 'certainty' can be reached by doing so - and leads to more use of stereotypes in decision-making. This is contrary to what Tiedens \& Linton (2001) found, namely that uncertainty-related emotions lead to less heuristic processing and less reliance on stereotypes. This, however, holds for a different kind of situation, individualized decision-making, i.e., situations, where belonging to a certain group does not play a role, and therefore, a state of subjective certainty cannot be reached by social identification. One could even imagine that uncertainty reduction via identification finally leads to a relatively high feeling of certainty, and feelings of certainty are positive emotions. A positive emotional state does not only lead to positive feelings about oneself and one's group, but also influences decision-making and information processing in that it leads to more heuristic processing (e.g., Bless et al., 1996; Bless \& Schwarz, 1999). Hogg and Hains (1998) find in experiments that strongly identifying small decisionmaking groups "faced with difficult decisions and much uncertainty produce overwhelming social attraction and unwarranted certainty" (Hogg \& Mullin, 1999, p. 267).

Turner et al. (1986) show that social-identity salience leads to greater perceived ingroup homogeneity (for related arguments see also Haslam et al., 1996). Identification with an ingroup also affects the perception of individual ingroup and outgroup members. The most important effect in our context is that it renders perceptions of outgroup members more stereotypical. This is essentially one way how identification with the ingroup reduces uncertainty: Enhancing stereotypicality, it makes the world appear less complex, and gives clear guidelines what is 'right' and 'wrong'. Hogg \& Mullin (1999), however, reject the idea that it is just search for simplification, which underlies the need for certainty, but rather assume a - not further specified - search for 'meaning'.

If people categorize themselves in terms of a salient ingroup, a general tendency to favor ingroup members (see Fiedler, 2000) and discriminatory behavior against members of an outgroup can result. This is again especially likely to happen when the identity of the group is threatened. Minimal-group studies have further shown that people not only favor their own groups, but that they sometimes even sacrifice maximum absolute ingroup gain to ensure a greater relative gain of their group (Spears 2001; see 
also Brewer, 1979; Tajfel et al., 1971; Turner, 1981). Especially high identifiers are prone to display ingroup-favoring behavior, or refrain from compensating behavior towards the outgroup.

Social identity becomes especially salient when the status relations between groups become unstable (Doosje, Ellemers \& Spears, 1999, p. 93), or when norms of different groups become too similar. This is given for labor market discrimination of women and blacks, as the relative status of these minority ${ }^{17}$ groups is changing recently and norms these groups hold with respect to work become more similar to those of the majority groups, endangering distinctiveness (Jetten, Spears \& Manstead, 1999). As people strive for a distinct social identity, similar norms (but also very different ones) between groups can lead to more discriminatory behavior against an outgroup, to protect the unique identity of the ingroup (see also Jetten, Spears \& Manstead, 1996, for an experimental study of this phenomenon). Jetten et al. (1999) found different effects of differentiability of groups depending on whether subjects where high or low identifiers and whether they were 'prototypical' members of their group. High identifiers/prototypical members showed more ingroup bias ${ }^{18}$, when norms were 'too' similar, whereas low identifiers showed more ingroup bias when intergroup norms were very different. In general, more prototypical members showed more ingroup bias when groups were threatened, while less prototypical members showed less ingroup bias in such a situation, thus distinguishing themselves from the group (Jetten Spears \& Manstead 1997 on prototypicality; Jetten, Spears \& Manstead, 1998 on strength of identification). Spears (2001) reports a similar kind of 'strategic' self-categorization: In an experimental study, low identifiers responded to identity threats to the ingroup by distancing themselves from the group (thereby avoiding the threat by focusing on their individual self), while high identifiers 'closed ranks' (Spears, 2001, p. 189; for details on the experiment see Spears, Doosje, \& Ellemers, 1999).

Decision-making on a group basis can also lead to the opposite of outgroup discrimination, outgroup favoritism. This happens if the stereotype of the other group is positive in some relevant aspect, and the related emotion is positive, or if there exist some learned status differences (see e.g. Spears et al, 2001).

Interindividual differences in the extent to which people are prone to uncertainty reduction by identification with a group have been reported as well. Hogg \& Mullin

\footnotetext{
${ }^{17}$ Women are of course not a minority in general, but in many occupations they still are.

18 Ingroup bias means showing a preference for the ingroup, be it cognitive, emotional or in behavior.
} 
(1999) distinguish between "uncertainty-oriented" people, who "work on the resolution of uncertainty in order to satisfy a self-assessment motive, whereas certainty-oriented people are more concerned with self-verification and the maintenance of existing beliefs - they avoid situations of uncertainty and if confronted by uncertainty fall back on simple heuristics rather than resolution of uncertainty [... $]^{\prime \prime}$ (p. 157) ${ }^{19}$. These interindividual differences are, however, not the focus of interest of this thesis.

\subsubsection{Identification in economics}

Not much economic research is dealing with identification up to now. Akerlof \& Kranton (2000) are among the few economists making use of that concept in an analysis of discrimination. They adopt a simple definition from psychology, identity as "a person's sense of self" (p. 715), and add it as one more variable to the utility function, stating that people derive utility from identifying with a certain group. Identity is supposed to prescribe certain behaviors and thereby can evoke discriminatory outcomes against some groups. An example would be that the prescriptive behavior for a female identity is rather being a housewife and not working, such that working implies an identity loss. Working alongside with women implies an identity loss for men, leading to a discriminatory taste against women.

Other examples of economic research on the role of identity are Ely (1995) analyzing women's problems with their identity when being in largely male firms, or Bloch \& Rao (1993) who developed a model that shows how minority-group members can opt for adopting behavior associated with the dominant group identity, and thereby ameliorate their situation.

\subsubsection{Summary and definition used here}

Stroebe et al. (1996) define social identity as a person's feeling about who or what he or she is, resulting from the belonging to a group (p. 631, own translation). The most prominent social-psychological theories dealing with identification and social identity are social-identity theory and its extension self-categorization theory. Both theories are based on the idea that a purely cognitive analysis of human behavior is incomplete, and that aside from cognitions and emotions the social context has to be taken into account as well. Identification and self-categorization in terms of a salient ingroup are seen as important links between stereotypical cognitions, prejudices and

\footnotetext{
${ }^{19}$ Self-assessment means, e.g., to figure out whether the own position is correct, while self-verification means to confirm that the own position is correct.
} 
behavior. Reasons for self-categorization in terms of a certain group are striving for selfesteem and reduction of uncertainty in one's life. The social context of the group plays an important role in determining which group and how strongly to identify with, and also how behavior is influenced by the identification. Identification renders perceptions of outgroup members more stereotypical and can lead to ingroup-favoring or outgroupdiscriminating behavior (in certain cases also to the opposite). Social identity becomes especially salient when the status relations or norm differences between groups become unstable. A few economists use identification in analyses of discrimination or related topics based on psychological definitions.

For the research conducted in this thesis, the basic concept from social-identity theory is used, i.e. social identity refers to: categorization in terms of and identification with groups one belongs to. The social identity is part of a person, in addition to the 'personal identity': Groups one is part of and identifies with are called 'ingroups', in contrast to 'outgroups'.

\subsection{The role of uncertainty}

Uncertainty is an important concept in the economic and social-psychological analysis of decision-making. It is discussed interdisciplinary in decision-making psychology and economics, but still seems to be more central to research in economics than in psychology, and therefore in the following first the economic use of the concept is discussed.

\subsubsection{Economics on uncertainty}

In economics, several concepts of uncertainty are distinguished, in part depending on research schools. General-equilibrium economics usually deals with uncertainty in the sense of imperfect information. The assumption of (rational) expectations or calculable risk (see below) allows analyzing situations of uncertainty within its core theoretical framework (cf. Rothschild, 1981). Examples are uncertainty about the behavior of competitors in oligopoly situations, asymmetric information (see for a seminal paper Akerlof (1970) on the market for lemons) or imperfect information as used in statistical discrimination models.

(Interdisciplinary) research on decision-making under uncertainty in economics and psychology is quite specific about the kind of imperfect information or uncertainty it 
is dealing with. Usually, a distinction is made between risk and ambiguity ${ }^{20}$ (Knight, 1921; see also Camerer and Weber, 1992). Risk is uncertainty about the outcome of a decision in a situation where the probabilities of possible outcomes are, at least subjectively, known. "Ambiguity is uncertainty about the probabilities, created by missing information that is relevant and could be known" (Camerer \& Weber, 1992, p. 330). Another distinction made especially by (neo-) Keynesian research is the distinction between ambiguity (in the above sense) and fundamental uncertainty, the latter being a situation where probabilities are not known and cannot be known in principle, because possible future developments cannot be anticipated. In the following, these three concepts will be described in more detail.

Risk is the concept the most prominent theory of choice, expected utility theory (EU; v. Neumann \& Morgenstern, 1947) deals with. In EU the utility of a choice is calculated as the expected utility of the possible outcomes weighted by their objectively known probabilities (see for an overview Camerer, 1998; Camerer \& Weber, 1992).

Subjective expected utility theory (SEU; Savage, 1954) does not assume that probabilities of outcomes are objectively known, but posits that people have subjective probabilities for all possible 'states'. Often, 'real' probabilities are assumed to be unobserved. Subjective probabilities are supposed to be revealed by the choices people make. By definition, subjective probabilities cannot be unknown to the individual. They may, however, be different across people. Thus, the distinction between known and unknown probabilities does not apply to SEU, and decisions can be treated as decisions under risk.

But, how much people know about the probability of a state has been found to influence whether they are willing to bet on that state: People are ambiguity averse. This has to be distinguished from risk aversion, wich is aversion against uncertain outcomes, while ambiguity aversion is aversion against uncertain probabilities (see, e.g., Ellsberg 1961; Viscusi \& Chesson, 1999; Ho et al, 2002). Camerer \& Weber (1992) give as an illustration the example of having the possibility to bet on one of two coins. One of them has been thrown a thousand times and proved to be fair. The other one has been thrown two times and showed once head and once tail. Even if most people would in general believe that both coins are fair, many would prefer to bet on the first coin, because it appears less ambiguous.

\footnotetext{
${ }^{20}$ Sometimes also called 'uncertainty'.
} 
Many different sources of ambiguous probabilities are possible: "Uncertainty about the composition of an urn of balls..." (as in Ellsberg's study) "... is just one kind of missing information. Feeling ignorant about football or politics, having doubts about which of several experts is right, wondering whether your child has a predisposition to the side effects of a vaccine, or being unsure about another country's economy are all manifestations of missing information. Some new studies suggest these other kinds of missing information about events make people reluctant to bet on the events" (Camerer \& Weber, 1992, p. 360). Explanations for ambiguity aversion range from the idea that not knowing important information is scary (Heath and Tversky, 1991), over the assumption that people use heuristics in decision making (Frisch \& Baron, 1988), to the argument that people strive for a feeling of competence (Fox and Weber, 2002). Camerer and Weber (1992) conclude that ambiguity aversion will make people value the provision of more information, if this information reduces ambiguity or enhances perceived competence - whether it will change the decision or not.

Fundamental uncertainty is defined by Dequech (2001) as a situation, where at the time of the decision some information does not exist, and cannot be known: "[...] situations of fundamental uncertainty are essentially characterized by the possibility of creativity and structural change and therefore by significant indeterminacy of the future. The future cannot be anticipated by a fully reliable probabilistic estimate because the future is yet to be created. [...] The institutional arrangements are such that competition stimulates decision makers to innovate in search for extra profits, which introduces an endogenous pressure for something that causes fundamental uncertainty." (Dequech, 2001, p. 916)

Fundamental uncertainty implies that waiting for more information before making a decision may not be worthwhile, because some information can never be obtained ex ante. The passage of time implies that fundamental uncertainty would even exist if people could compute everything they want using the information at hand, because there is still information, which cannot be known at the moment of decisionmaking. Fundamental uncertainty is therefore not the same as complexity, because in a complex situation one can imagine all information to be knowable in principle. Fundamental uncertainty, however, is also not the same as complete ignorance. The existence of fundamental uncertainty is seen by many neo-keynesian economists as one main argument against neoclassical general equilibrium models, which cannot deal with such uncertainty other than by assuming it away and transforming it into risk (see 
Rothschild, 1981). This, in their view, does not meet the character of uncertain expectations, which (i) can be subjective, and (ii) often cannot be calculated. But, of course, it is far more difficult to deal with such fundamental uncertainty in a formal way (see Rothschild, 1981, p. 124).

Theories like statistical discrimination, which are rooted in the neoclassical approach, do not make such a clear-cut distinction between risk, uncertainty, and fundamental uncertainty when talking about 'uncertainty', but rather use it in the general sense of imperfect information. Assuming that probabilities of productivities of applicants are at least subjectively known, this imperfect information is operationalized as risk.

\subsubsection{Social psychology on uncertainty}

One stream of decision research in social psychology is closely linked with economic decision research, as described before. There is a difference in specific areas of interests between economists and psychologists in this field, but in general the concepts used are the same.

In social-identity theory, a conceptualization of uncertainty different from the ones described up to now is used: self-relevant subjective uncertainty. This refers to subjectively felt uncertainty about things, which are important for one's life. It might be uncertainty about a job, about the personal situation etc. In this tradition, uncertainty is rather seen as an aversive emotional state, associated with emotions like unease or even fear, "[...] because it is ultimately associated with reduced control over one's life" (Hogg \& Mullin, 1999, p. 253). (Self-relevant) subjective uncertainty is said to be evoked by the (social) context, e.g. "[...] threats that arise from geographical relocation, rapidly changing status differentials, loss of membership or loss of validation of membership in a group [...]. Sudden loss of work-related identity through retrenchment or retirement can have similar effects" (Hogg \& Mullin, 1999, p. 266). Among the situations, which evoke (self-relevant) subjective uncertainty, Hogg \& Mullin (1999) mention situations of strengthened economic competition. The concept of competition here is used in the way colloquial language uses it, not as an economic technical term (see section 2.7.2).

Hogg \& Mullin (1999) generally assume that self-relevant subjective uncertainty includes some 'deeper' uncertainty, related to one's self-concept, but in their operationalization of the concept for experimental studies they do not refer to such 'deeper' uncertainty, and just use situations of subjective, but hardly self-relevant, 
uncertainty: Minimal-group studies are assumed to represent situations of high subjective uncertainty, because they are new situations to the subjects and the task probably seems strange (Hogg \& Mullin, 1999). (Self relevant) subjective uncertainty is linked to discriminatory behavior, because it is assumed to enhance identification with a salient ingroup, which then reinforces discriminatory attitudes and behavior (see section 2.5.2).

Even if, on first sight, it looks as if this concept of '(self-relevant) subjective uncertainty' has not much in common with the economic and decision-theoretic concept of uncertainty, taking a closer look, commonalities can be found. (Self-relevant) subjective uncertainty arises obviously from uncertainties in the environment, or at least from their subjective perception. Decision researchers like Camerer (1998) also mention that ambiguity is accompanied by aversive feelings (see above). This, of course, also holds for risk, even if aversive feelings might be weaker under risk than under ambiguity, and it holds for fundamental uncertainty, where aversive feelings might be stronger.

One can therefore interpret self-relevant subjective uncertainty as consisting of a subjective perception of ('objective') uncertainty accompanied by an aversive emotional state, which becomes stronger, the more self-relevant the source of uncertainty and the higher the level of 'objective' uncertainty is.

\subsubsection{Summary and definitions used here}

Uncertainty is an important concept in the economic and social-psychological analysis of decision-making. Interdisciplinary research on decision-making distinguishes between risk, ambiguity, and fundamental uncertainty, which are distinguished by whether outcomes or probabilities are uncertain and whether the latter probabilities can be known. Standard (neoclassical) microeconomic theory usually uses uncertainty in the sense of risk, where probabilities are objectively (or subjectively) known and thus can be treated within a general-equilibrium framework assuming, e.g., rational expectations.

In social-identity theory, another conceptualization of uncertainty is used: '(selfrelevant) subjective uncertainty'. This concept does not deal with the question how much of the information is known, but rather with the psychological effects of missing information. It mainly refers to subjectively felt uncertainty about important things in life. (Self-relevant) subjective uncertainty induces self-categorization and identification and thereby can lead to discriminatory behavior against outgroups.

In the following, we will talk about risk when there is uncertainty about the state of the world but it is assumed that probabilities are known. We will talk about ambiguity 
when referring to Camerer and Weber's (1992) concept of "uncertainty about probability, created by missing information that is relevant and could be known" ( $p$. 330). Self-relevant subjective uncertainty is defined as a subjective perception of ('objective') uncertainty accompanied by an aversive emotional state, which becomes stronger, the more self-relevant the source, and the higher the level of 'objective' uncertainty is. It can pertain to all kinds of uncertainty, with growing strength: Other things being equal, risk evokes weaker feelings of subjective self-relevant uncertainty than ambiguity and fundamental uncertainty.

\subsection{The role of competition}

As competition also is a concept which is more prominent in economic than in psychological research on discrimination, in the following first the economic and secondly the psychological definition of the concept is discussed. But, to start with, a more general definition of competition, taken from the Oxford advanced learners' dictionary is given, to show how economic and psychological uses of the concept relate to such a definition. The dictionary describes 'competition' as "activity in which persons compete," and to compete in its turn is defined as "take part in a race, contest, examination etc.". Competition in this basic definition is thus seen as an activity, taking place between different subjects, to reach a certain goal, which can only be reached by some or even one of them, e.g., winning a medal in a race. We now turn to the question, how economics and social psychology interpret this concept.

\subsubsection{Economics on the role of competition}

Competition is one of the most important concepts in an economic analysis of discrimination. However, there are different ways of defining the concept, depending on research schools (see for a discussion Zafirofsky, 2002). The basic idea of economic competition is the same as described in the lexicon article: As many goods are scarce, economic agents have to compete for them. Markets evolved as institutions where this takes place and where prices for these goods develop. Markets for scarce goods can take different forms, depending, among others, on the number of competitors, on how the good can be divided, etc., i.e., on the specific form this competition takes. The form of competition also determines the (perceived) strength of competition in a market - e.g., the more competitors there are and the smaller the chance is to get the desired result or amount of a scarce resource, the stronger competition is (perceived). 
Among the different forms competition can have, one of the most important (benchmark) concepts of today's mainstream economic research is 'perfect competition'. The textbook definition of a perfectly competitive industry illustrates this concept (Nicholson, 1998): "A perfectly competitive industry is one that obeys the following assumptions: 1) There are a large number of firms, each producing the same homogeneous product. 2) Each firm attempts to maximize profits. 3) Each firm is a price taker: It assumes that its actions have no effect on market price. 4) Prices are assumed to be known by all market participants - information is perfect. 5) Transactions are costless: Buyers and sellers incur no costs in making exchanges" (p. 401f). This conception on first sight deviates in part from the dictionary definition: There is nothing said about an activity of competing, but it rather describes a static situation and different features characterizing it. There are, however, hints about a competitive activity, namely conditions 2 and 3 . Condition 2 states that firms try to maximize profits, which goes at the expense of other firms' profits. Thus, it comes close to part of the dictionary definition. Condition 3 implies competitive activities as well, however, rather pricesuppressing activities of the other participants in the market.

The focus in neoclassical general equilibrium models, which often use perfect competition as a benchmark, is on the existence and characteristics of equilibria, which markets can reach when they fulfill certain conditions, and not so much on how people precisely act in such a market. This becomes also obvious in that firms are treated as 'black boxes', holistic entities, and not as consisting of human beings with cognitions, emotions, motivations, and specific behaviors. Human beings enter these models usually as rationally calculating agents, and firms' behavior is not seen as differing from the behavior of single entities. Of course, there are exceptions to such models, and in recent times, e.g., research in 'behavioral economics' as mentioned in section 1.1, introduces more and more 'human' aspects into economic analysis.

A perfectly competitive equilibrium can be a model for many relationships found in real markets, but captures process aspects of competition only implicitly, in that it makes a distinction between short-run and long-run equilibria and then analyses comparative statics of such short-run or long-run equilibria. The processes, by which such equilibria are reached (or not), are not investigated in most of the economic research in that tradition (Friedman \& Sunder, 1994).

Aside from analyzing perfecly competitive equilibria, many (neoclassical) economic models deal with situations of imperfect competition, e.g., monopolies, 
oligopolies, or markets with imperfect information. Situations of imperfect competition most basically are competitive situations where not all the above conditions are met. The main focus is on the analysis of possible equilibria in various situations and of how they differ from perfectly competitive equilibria (for (critical) discussions of equilibrium economics see, e.g., Rothschild, 1981; Zafirofsky, 2002).

Blaug (2001) discusses the mainstream way of dealing with competition, arguing, that economics in former times in fact had a conceptualization of competition much closer to the dictionary definition. Economics, he argues, historically had two different "notions of what is meant by competition: [...]". Either an "end state of rest in the rivalry between buyers and sellers", as in the perfect-competition model, or ".... a process of rivalry that may or may not terminate in an end state". Blaug himself favors the process conception, which he describes as seeing competition as "[...] an active process of jockeying for advantage, tending towards, but never actually culminating in, an equilibrium end-state" (p. 37f.). It is basically the idea that competition is an activity of people that inspires an incorporation of psychological variables into an (equilibrium) analysis of, e.g., effects of competition on discrimination in the labor market.

Effects of competition (in the sense of the end-state definition) are analyzed in both economic theories of discrimination described here. In general, (perfect) competition is assumed to eliminate 'irrational', not profit-maximizing behavior of firms. An important question in both discrimination models therefore is what happens to discrimination when competition strengthens and/or reaches the long-run competitive equilibrium.

In Becker's model of employer discrimination, the analysis of the effects of competition on discrimination has a central place. As described in section 2.4.2, the model assumes that discriminating employers have a 'taste for discrimination', i.e., they are willing to forego profits when they can avoid working with a certain kind of workers. However, under perfect competition, and if all firms had the same production function, firms that discriminated less would always have lower unit production costs than firms that discriminated more, since the former firms hire a larger number of less expensive, but equally productive outgroup members. Hence, in a competitive product market, less discriminatory firms have a competitive advantage. In the case of constant unit costs, this enables them to expand and drive the more discriminatory firms out of business in the long run. Alternatively, when unit costs rise with output beyond the unit-cost-minimizing point, less discriminatory employers could buy out more discriminatory employers, or 
start new plants and thus gradually take over the market. ${ }^{21}$ As a result, the demand for outgroup labor would increase and the market wage gap would be reduced. If the least discriminatory employer has a zero discrimination coefficient, the market wage gap would even become zero, i.e. discrimination would be eliminated. Buying out a more discriminatory employer can also occur when the employer has monopoly power which is transferable (Becker, 1971). However, many monopolies are non-transferable and buying out may not always be profitable (Ashenfelter and Hannan, 1986).

Even in the case of competitive markets it can be noted that in practice a long-run equilibrium in which the more discriminating firms have left the market because of losses may never be reached because of remaining entry barriers. ${ }^{22}$ On the other hand, long before discriminating employers could be driven out of the market by less discriminating employers, strengthening competition may urge the former employers to diminish their discriminatory behavior. This income effect of falling profits ${ }^{23}$ is easily explained as follows. Assume that following one's discriminatory taste is a normal good. When profits, and thus income, go down (for simplicity, assume with Ashenfelter \& Hannan (1986, p. 154) an owner-manager), for example because of market entry of more firms, less of this normal good will be consumed, and thus discriminatory behavior diminishes.

Overall, the preceding analysis predicts that as the product market is more competitive, employer discrimination will be weaker (c.p.). ${ }^{24}$ On the other hand, in Becker's theory there are other kinds of discrimination, which may not diminish so easily under competitive pressure, and which often accompany employer discrimination. One of these is employee discrimination, as described in section 2.4.2. If ingroup employees receive fixed wages independent of profits, employee discrimination will not diminish under competitive pressure since there will be no income effect of falling wages and since profit-maximizing employers have to take into account the discrimination by their employees. On the other hand, if employees (also) receive profit shares, they will be more sensitive to the economic advantages of hiring cheap outgroup members. When competition becomes tougher, their discrimination coefficient may then diminish by an

\footnotetext{
${ }^{21}$ Surprisingly, Becker (1971) and Becker (1993) do not mention the latter two options for the case of competitive industries. Becker (1971) mentions the option of buying out discriminatory employers for monopolistic industries only.

${ }^{22}$ The competitive market then gets stuck in a long-run equilibrium, which is similar to a short-run equilibrium with positive profits in the perfect-competition model.

${ }^{23}$ See Comanor (1973, p. 372) and Ashenfelter and Hannan (1986, p. 154)

${ }^{24}$ See Ashenfelter and Hannan (1986) for two more arguments for this prediction.
} 
effect similar to the income effect discussed above. If, however, under competitive pressure fears for unemployment increase among ingroup employees, they may become afraid of being replaced by cheap outgroup members. This may raise their discrimination coefficient even if they receive profit shares.

Borjas and Bronars (1989), Black (1995), and Bowlus \& Eckstein (1998) show how search costs, either for firms or for workers, can lead to persistent discriminatory outcomes, even when competition strengthens, when only some employers are unprejudiced: Outgroup workers are assumed to have higher search costs than ingroup members, because they have to find an unprejudiced firm, which makes longer search necessary. Search costs also prevent total segregation of the market. Only if an unlimited number of possible employers exist, entry will eliminate discrimination in Black's (1995) model. Bowlus and Eckstein (1998) focus on search by firms, and come to similar results. Without equal-opportunity laws, firms might reach complete segregation of the market in their model.

Statistical discrimination is a model of imperfect information, i.e., of imperfect, but pure, competition. It analyzes what happens to discrimination when the economy approaches the competitive equilibrium (with imperfect information), in the case when the perceptions of groups leading to discriminatory behavior are correct and in case they are wrong. In the latter case, the discriminated group will try to provide better measures for their productivity, and so in the long run information will become better and discrimination will diminish or disappear. If, however, the (wrong) perception originates from a personal prejudice and serves as its rationalization and justification, as described in section 2.3.2, an increase in competition does not lead to a change in perceptions. In that case, the same mechanism as in the model of Becker applies with respect to discriminating firms - they will be competed out of the market.

In the former case, when perceptions are correct, under competitive pressure (for example, when more firms enter the market) discrimination will not be suppressed, as it is economically rational behavior to hire members of groups which are on average more productive, when there is no more diagnostic information about individual applicants available. Statistical discrimination in that case is rational from a profit-maximizing point of view.

A combinination of a taste-based concept with statistical discrimination provides an interesting case in which the strengthening of competition reinforces discrimination. This is the case when initially no actual difference in average productivity between the 
ingroup and the outgroup exists, but employee discrimination reduces the marginal productivity of outgroup members directly as well as indirectly by lowering the productivity of discriminating ingroup members (see section 2.4.2).

\subsubsection{Social psychology on the role of competition}

Social psychology does not handle an explicit definition of competition. In general, if the term is used, it is used like in everyday speech, i.e., like the dictionary definition, indicating rather an activity of competing. Research on discrimination mainly discusses intergroup competition - either as a tool to reduce prejudice within a group consisting of members of different (sub-) groups (see, e.g., Sherif et al. 1961) ${ }^{25}$, or in analyses of how competition of groups influences identification with the ingroup and related variables (e.g. Bornstein et al., 1996). Sport psychology is dealing with the influence of competition on athletes (e.g., Anshel, Jamieson \& Raviv, 2001). Van de Vliert (1999) discusses whether competition (again seen as an activity) is the contrary of or related to cooperation. Hogg \& Mullin (1999) mention economic competition as one reason for self-relevant subjective uncertainty (see section 2.5.1), but without defining more clearly what they mean by competition in that case. It can be assumed that they use the term in a manner everyday speech uses it when speaking of economic competition, i.e. in the sense of a rather tough competitive activity going on in the economy.

\subsubsection{Summary and definition used here}

If neoclassical economists talk about (perfect) competition, their use of this word deviates from the concept known from everyday speech. They usually mean by (perfect) competition, that a large number of firms producing an homogeneous product try to maximize profits. Firms are price takers, information is supposed to be perfect and there are no transaction costs. Neoclassical economics also analyses situations of 'imperfect competition', e.g., monopoly or situations with imperfect information. However, apart from this 'end-state' concept of competition, economics also knows a 'process' notion of competition, i.e., competition is - mainly by economists from the 'Austrian' school - seen as a process of rivalry.

Both economic discrimination theories reviewed here deal with the effects of competition, based on the neoclassical concept. In Becker's model, information is complete and discrimination results from 'irrational' tastes. Strengthening competition

\footnotetext{
${ }^{25}$ Sherif et al. (1961) showed that groups who formerly disliked each other became friends when cooperating in competition against another group.
} 
therefore will eliminate employer discrimination in the long run. Employee- or customer discrimination, on the other hand, does not necessarily disappear when competition strengthens.

Statistical discrimination theory assumes imperfect information. Discrimination is supposed to persist when competition strengthens, if groups really differ in some important characteristics. Otherwise, groups will provide better information and discrimination will disappear in the long run. When perceptions of differences are rationalizations of discriminatory tastes, the same mechanism as in Becker's model will apply.

Social psychology uses the term competition generally like in everyday speech, indicating the activity of competing. Hogg \& Mullin (1999) mention economic competition as one reason for self-relevant subjective uncertainty, which can lead to more discrimination.

A link between an economic and a psychological conceptualization of competition has to rely on an activity conceptualization in so far as psychological aspects of human behavior and interactions have to be considered. However, this does not imply that an equilibrium approach is useless for the research here. There is no contradiction between analyzing equilibria and the assumption that competition also implies an activity of people. An equilibrium can be interpreted as a situation in which activities have settled at a stable level. Thus, one can emphasize that in a perfectly competitive situation people are engaged in a certain activity. This makes it logical to look also at how their psychological 'states' are influenced by the situation and how this in turn influences their behavior. The approach taken here is not interested in what long-run equilibrium the economy reaches. It assumes however, that 'individual' equilibria in the sense of utility maximization as well as short-run market equilibria are possible, and that the former are influenced by the level of competition in the latter market equilibria. Therefore, it conceptualizes competition as an activity performed by firms or other economic agents, who engage in this activity against each other to reach certain goals.

In the following table the core concepts as defined by (social) psychology, economics, and as chosen for the research here are summarized. 


\begin{tabular}{|c|c|c|c|}
\hline & Economics & Social psychology & Definitions chosen \\
\hline Stereotypes & $\begin{array}{l}\text { A (wrong or correct) perception of a } \\
\text { group average, which is then applied } \\
\text { to judge members of that group. }\end{array}$ & $\begin{array}{l}\text { Common beliefs about personal } \\
\text { attributes and behavior of } \\
\text { members of a group. }\end{array}$ & $\begin{array}{l}\text { Stereotyping is "...the unconscious or conscious application } \\
\text { of (accurate or inaccurate) knowledge of a group in judging } \\
\text { a member of the group" (Banaji \& Greenwald 1994, p. 54). }\end{array}$ \\
\hline Prejudice & $\begin{array}{l}\text { 'Taste' against associating with a } \\
\text { particular group. }\end{array}$ & $\begin{array}{l}\text { The emotional aspect of } \\
\text { discrimination. }\end{array}$ & $\begin{array}{l}\text { Prejudices are negative, affective attitudes towards all or } \\
\text { most members of a certain group (cf. Pettigrew, 1997). }\end{array}$ \\
\hline Identification & $\begin{array}{l}\text { A person's sense of self (taken from } \\
\text { psychology). }\end{array}$ & $\begin{array}{l}\text { A person's feeling about who or } \\
\text { what he or she is, resulting from } \\
\text { the belonging to a group. }\end{array}$ & $\begin{array}{l}\text { 'Social identity': Identification with and categorization in } \\
\text { terms of groups one belongs to. It is part of a person, in } \\
\text { addition to the 'personal identity'. Groups one is part of and } \\
\text { identifies with are called 'ingroups', in contrast to } \\
\text { 'outgroups'. }\end{array}$ \\
\hline Uncertainty & $\begin{array}{l}\text { Imperfect information - } \\
\text { a) Risk (uncertainty about state) } \\
\text { b) Ambiguity (uncertainty about } \\
\text { probabilities) } \\
\text { c) Fundamental uncertainty } \\
\text { (probabilities are not known and } \\
\text { cannot be known). }\end{array}$ & $\begin{array}{l}\text { a) Imperfect information - } \\
\text { probabilities of possible } \\
\text { outcomes are not known } \\
\text { (ambiguity) } \\
\text { b) "(Self-relevant) subjective } \\
\text { uncertainty" - subjectively } \\
\text { perceived uncertainty about } \\
\text { things, which are important for } \\
\text { one's life, accompanied by an } \\
\text { aversive feeling. }\end{array}$ & $\begin{array}{l}\text { a) Risk: Uncertainty about the state of the world, it is } \\
\text { assumed that probabilities are known. } \\
\text { b) Ambiguity: "Uncertainty about probability, created by } \\
\text { missing information that is relevant and could be known" } \\
\text { (Camerer and Weber's ( } 1992 \text { ), p. 330). } \\
\text { c) Self-relevant subjective uncertainty: A subjective } \\
\text { perception of ('objective') uncertainty accompanied by an } \\
\text { aversive emotional state, which becomes stronger, the more } \\
\text { self-relevant the source, and the higher the level of } \\
\text { uncertainty is. It can pertain to all kinds of uncertainty, with } \\
\text { growing strength. }\end{array}$ \\
\hline Competition & $\begin{array}{l}\text { a) An activity of competing in which } \\
\text { economic agents engage. } \\
\text { b) An equilibrium end-state, fulfilling } \\
\text { certain conditions. }\end{array}$ & $\begin{array}{l}\text { The activity of competing in } \\
\text { which people engage. }\end{array}$ & $\begin{array}{l}\text { An activity performed by firms or other economic agents, } \\
\text { who engage in this activity against each other, to reach } \\
\text { certain goals. }\end{array}$ \\
\hline
\end{tabular}

Table 2.1: Summary of the core concepts as defined in each of the two disciplines and the definitions chosen 


\subsection{The general integrative framework}

\subsection{Conceptual aspects}

Before discussing possible contributions and problems of an integration, it is important to define what is meant by integration in this context.

Mamuth (1993) describes theoretical models of different disciplines as 'abstract maps', which are useful to answer the questions relevant for each discipline. These 'maps' in his view cannot be used by different disciplines together, as they are organized in a specific way to answer the questions each specific discipline has. If this is the case, how could an integrative approach be possible at all? The idea of this project is, while being aware of the fact that for many questions in psychology and economics different 'maps' are necessary and useful, there are certain questions where at least part of the answer can be found on a common 'map'. Econometric studies about the wage gap for example are on a different level of aggregation than experimental psychological studies, i.e., they use differently scaled maps. But, if the question about discrimination in the labor market is asked in a way where the level of aggregation is the individual in interaction with its environment, psychology and microeconomics do in fact use a 'map' of the same scale.

This thesis tries to integrate economic and social-psychological research on discrimination in the labor market with respect to concepts used and the methodology applied (see also section 2.8.2). With respect to concepts, integration means that the ideas both disciplines have about discrimination - on the level of the individual in interaction with its environment - are analyzed to find out what they have in common and where they differ, as has been done in this chapter. Doing this, an attempt is made to construct a conceptual framework for an explanation of discrimination in the labor market using contributions of both disciplines in a way that allows gaining new insights. This will lead to a rather broad integrative framework, parts of which are then transformed into a coherent formal model. Parts of this model and of the general framework are submitted to empirical testing; making use of (quasi-) experimental techniques borrowing from the methodological traditions of both disciplines.

Another 'integrative' aspect is the integration of different concepts within the same discipline. As has been said before, especially social psychology is a very scattered discipline. The integrative framework and the theoretical model constructed here also aim at unifying some of these divergent ideas in one model in as far as this is possible. 
This chapter showed that definitions of the concepts important for an analysis of discrimination differ within and between the disciplines. However, there are also many similarities, so that it seems possible to bridge these differences at least partially. This is what the integration firstly aims at. It further assumes that in an analysis of the effects of competition on discriminatory behavior of employers and employees not only 'economic' mechanisms play a role, but also 'psychological' mechanisms, especially if one focuses on (strengthening) competition.

It may be useful to say a few words about the use which is made here of the concept of 'ceonomic'. 'Economic mechanisms' here refers to a restricted concept of 'economic', dealing with 'financial-economic' rationality, i.e., constrained utilitymaximization with respect to profits. Therefore, if we speak about the 'economic effect of competition', such a financial-economic effect is meant. Micro-economic rationality is a much broader concept, referring to utility maximization under constraints more in general. This can comprise motives like a discriminatory taste, which are 'irrational' from the point of view of financial-economic rationality. This thesis uses the narrow concept of financial-economic rationality for pragmatic reasons, to be able to distinguish between 'psychological' and 'economic' mechanisms in an intuitively appealing way.

The two main economic theories explaining discrimination in the labor market, Becker's 'taste' approach and statistical discrimination, both use 'psychological' variables as explanatory variables for the existence of discrimination, but in a static sense and interpreted as exogenous. The concept of competition they work with does not take into consideration how the 'activity' or the 'process' of competing might influence these psychological variables and thus preferences of subjects involved in this activity. In fact, such questions are usually more the focus of psychological research, but answers to them are important for economics as well, especially if one is also interested to see what happens when there is uncertainty and markets are not in long-run equilibrium (cf. Rothschild, 1981, p. 124).

In both economic frameworks, several possibilities are discussed how competition influences discrimination and how discrimination in turn can be persistent even when competition becomes stronger (see section 2.7). In Becker's framework, this is especially possible when discrimination comes from the side of employees or customers, or when there is no employer with a zero discriminatory taste. In the framework of statistical discrimination, this is only possible when perceptions of productivity differences are, or become, true. 
The review of psychological literature has shown that stereotypes and prejudices influence behavior context-specific. This implies that competition might influence whether and how these factors translate into discriminatory behavior. For example, competition can become stronger by an increase in the number of competing firms in the market. Under the general assumption of imperfect information, this can lead to a situation of strong (self-relevant) subjective uncertainty for members of individual firms, which already are in the market, since the risk of going bankrupt increases. To reduce this aversive feeling of uncertainty, people in firms can turn to stronger identification with their most salient ingroup - which could, for example, be white males. This, in its turn, strengthens the use of stereotypes and prejudice in decision-making and can in the end lead to more discriminatory hiring. That way the assumption of endogenous stereotyping and use of prejudice, influenced by competition, leads to predictions about the development of discrimination when competition strengthens, which are different from those of standard economic models. It is important to note that the research reported here is interested in the question what happens to discriminatory behavior when competition between agents increases, i.e., when the economy is becoming more competitive, but has not yet reached the end-state of a long-run competitive equilibrium. It is not of interest here, precisely what kind of equilibrium the economy reaches in the long run.

The main difficulty with such an integration of approaches from two disciplines lies in the fact that both use similar concepts differently. Therefore, this chapter tried to develop inclusive definitions of the most important concepts, by comparing definitions of economics and social psychology. For the theoretical and empirical research reported in the next chapters some of these concepts had to be operationalized. How this is done will be described in each chapter in detail, but first, section $\mathbf{2 . 8 . 2}$ deals with more general methodological questions.

\subsubsection{Methodological questions}

One important aspect in which this thesis deviates from standard economic approaches to analyze discrimination is the methodology applied. As has been shown, an important part of economic methodology to approach discrimination in the labor market consists of formal modeling, building on a strong, coherent theoretical framework. In (social-) psychology, such techniques are hardly applied at all. A lot of rather short- 
ranged theoretical models exist next to each other, which are mainly judged by how well they explain empirical data, and not, in how far they fit with any existing theoretical core.

Most empirical economic studies use econometric techniques to estimate, e.g., the size of the wage gap or the amount of segregation, as described in section 2.2. The main problem with these methods is that the unexplained residual, which is usually interpreted as stemming from discrimination, even with today's sophisticated regression analytic techniques, depends heavily on model specifications, as has been described in section $\mathbf{2 . 2}$ (c.f. Stanley \& Jarell, 1998). Only a few empirical economic studies explicitly test the main theoretical models, usually also using econometric methods. That this is so rarely done results in part from the fact that many of the variables used are difficult to operationalize and measure validly. Singell \& Thornton (1997), for example, see a methodological problem for testing Becker's model in the fact that it is empirically impossible to decide whether someone is utility maximizing or only profit maximizing. Such differences in part result from the fact that, using real-world empirical data, it is more or less impossible to manipulate only certain variables keeping others constant, to test for the influence of changes in specific variables. Many factors in the 'real world' cannot easily be disentangled. This is better possible in experimental studies, but at the cost of less external validity (see below). However, few economic studies on discrimination use experimental or quasi-experimental methods, and most of them mainly focus on the question whether there is discrimination (in hiring) at all (see section 2.2).

Empirical psychological research, on the other hand, mainly relies on laboratory experiments. Social-psychological research in general does not refer to one unified theoretical model, but consists of many small- and middle-range theories, which are tested extensively in experiments. The advantage hereby is the possibility to directly test for the specific relationships assumed: Certain variables, which are assumed to influence the outcome, can be varied in the laboratory, keeping all other variables constant. A disadvantage is, of course, that results from laboratory experiments cannot easily be generalized to human behavior outside of the laboratory.

Each of the two disciplines thus relies mainly on a certain methodology with its specific advantages and shortcomings. Somehow surprisingly, as at least part of social psychology and eonomics are interested in similar topics, there is not much methodological overlap in research on discrimination. Economics more recently started 
to use experiments as well, but up to now hardly to analyse discrimination in the labor market.

There are some important differences between experimental economics and experimental social psychology, which do not only pertain to the different focus of interest. Experimental economics is most interested in testing predictions of different theoretical approaches against each other, and in testing whether in specific situations certain equilibria are reached. Social psychology rather is interested in observing fundamental cognitive or social processes. Both disciplines sometimes use experiments in an exploratory way.

Apart from the differences in questions of interest, there are methodological differences between economic and social-psychological experimentation. While economists are convinced that it is necessary to provide real monetary incentives, psychologists often assume that it is enough to ask subjects to 'do their best' to motivate them. Secondly, economists try to model the context of interest as closely as possible in experiments, e.g., by giving subjects their utility functions in the experiment. Psychologists rather look at how people behave without such specific restrictions. This latter difference mainly results from the different focus of interest both disciplines have. Psychologists are more interested in general, universal patterns of human behavior, while economists are mostly interested in behavior of people in specific 'economic' situations.

A third important difference is the attitude towards deception. While psychologists think that - if subjects are carefully debriefed at the end - deception is acceptable in situations where it is necessary to answer a certain question, e.g., in discrimination research to avoid that social desirability concerns strongly influence behavior, economists in general see deception as inacceptable. This does not only have ethical reasons, but also is based on the idea that subjects should not become suspicious of being deceived in experiments, because this could influence their behavior (see e.g. Friedman \& Sunder, 1994, p. 132ff, Hertwig \& Ortmann, 2001, provide an exhaustive discussion of differences between the two disciplines in experimental methods).

A last, less important difference lies in the terminology used. This difference becomes important when experimental economists and experimental psychologists try to explain their experiments to each other. For example, what economists call 'treatment' is called 'condition' in experimental psychology. There are more such examples, but to report all of them would lead too far here. In the following, the the terminology of 
experimental economics is used, even if the experimental methodology borrows from both traditions.

This thesis not only integrates economic and psychological models, it also partially integrates methodologies. The main aims hereby are: (i) To describe a general framework of economic and social-psychological research on discrimination in the labor market. (ii) To develop a theoretical (utility-) model of discrimination in the labor market, using concepts of economics and social psychology, and thus creating a concise, formal model of a circumscribed situation, (ii) to submit some of the predictions of both the general framework and the formal model to experimental and quasi-experimental testing.

The choice for using (quasi-) experiments rather than field data was mainly made because it is very difficult to analyze the process of discrimination in the labor market using field data. First, several comparable situations have to be found which can be used in such a test. Secondly, it is necessary to measure discriminatory behavior and also some of the motives assumed to determine such behavior. This is very difficult to achieve in a real-world setting, as people know that discriminatory behavior is not socially desirable. Therefore, they rather do not report it, even if they are aware of it - which is not always the case either. Thirdly, in field settings it is often not possible to keep certain variables constant while changing others, to test only for some relationships between variables.

Of course, using experiments does not solve all methodological problems. In experiments as well, variables have to be operationalized and measured, and similar problems of measurability or of finding useful proxies for certain concepts as in other methods arise. The operationalization of the variables for the experiments used here always tries to capture the concepts of importance as well as possible, but this is always open to interpretation, as it is in other methods. The use of experimentation also does not completely solve the problem of social desirability, but one can construct experiments in a way that reduces social-desirability problems as much as possible. A last problem with experimental research is the question of external validity: Experimental results always are derived in 'artificial', laboratory settings, which differ in important aspects from the real-life settings they are supposed to model. Participants are usually students or other easily available groups, which are not necessarily representative for the population in question - and which often also acquire some experience in doing experiments. Even if important factors like utility functions are given, the situation in experiments is always a 'scenario', where participants have to imagine that they, e.g., want to maximize some 
utility function. One can doubt whether reactions in such a scenario are the same as when participants really are in a certain situation.

The clear advantage of using experiments lies in the fact that it provides a controlled setting, where the variables of interest can be analysed relatively free from the influence of other factors. They can be manipulated in ways, which are often not possible in real-life settings. That way, it can be known quite well what happens in a situation and what factors do influence behavior in what way. In contrast to field studies, it is even possible in experiments to establish causality or at least the direction of a relationship between variables.

To test some predictions of the model at least in a slightly more realistic setting. in this thesis use was made of a business simulation-game as a 'quasi-experimental' test. Of course, this goes along with a loss of control. Still, it would be desirable to have additional empirical evidence from the real world, but this goes beyond the scope of this thesis.

The advantage of using different methods to approach the same topic lies in the fact that each method has different shortcomings and advantages, which have to be traded off against each other, when only one method is chosen. Using different methods to analyse the same topic, in the end a clearer picture of the phenomenon analyzed can result. This of course only holds if the question to be answered lends itself to an analysis with different methodologies equally well, each of the methods is applied carefully, and comparability of the results is critically examined. This does not mean that for example the empirical (experimental) designs applied have to be one-to-one translations of the theoretical model, and the theoretical model has to be a one-to-one transformation of the framework of economic and psychological ideas into one formal model. In modeling as well as in designing experiments one has to make certain simplifications. Doing this, the aim still is to capture the most important aspects as well as possible. For example, treating competition as an activity does basically imply here that in the model certain important variables like stereotypes and prejudices are treated as endogeneous and are allowed to change, and in the experiments, subjects engage in competitive activity.

\subsubsection{An integrated framework}

The general framework resulting from an integration of economic and psychological research on discrimination in the labor market is shown in figure 2.1. The figure shows many possible relationships between more 'economic' and more 
'psychological' factors influencing discrimination in the labor market against outgroups or negatively stereotyped groups. The framework sketched here is very unspecific and just intends to show what kind of relationships can be thought of when combining economic and social-psychological insights. The following chapters will each select some of the relationships and analyse them more specifically.

The lower part of the graph shows rather 'economic' mechanisms, while the upper part shows rather 'psychological' mechanisms. Altogether, the graph shows how 'economic' and 'psychological' mechanisms could interact. A more 'economic' mechanism is the effect an increase in competition has on discrimination via enhancing the marginal utility of profits and risk aversion. Assuming that there are no real differences between the two groups in question, competition should then reduce discriminatory behavior, as it makes it more costly in terms of (monetary) utility. However, an increase in competition can also enhance 'objective' (see Rothschild, 1981) and 'subjective' uncertainty, since it raises the risk of going bancrupt. This can either lead to more information search about possible applicants or to identification with a salient ingroup - which has different effects on discrimination. Psychologically seen, an increase in the activity of competition can also directly enhance identification with the ingroup (see, e.g., Sherif et al. 1961) - with the same effects on discrimination: stronger use of stereotypes and prejudice and thereby enhancement of discrimination. However, in real-life settings different stereotypes about the same group can become salient at different times or even at the same time. Feedback effects from discriminatory outcomes on the discriminated group can further complicate the picture.

The following chapters are going to analyse some aspects of this framework: The theoretical model developed in chapter 3, which incorporates a large part of the framework, aims at being a first step towards a formal analysis of discrimination in the labor market using psychological concepts as endogenous variables. Economics provides the formalized framework, which allows for a more rigorous analysis than standard social psychology is used to. Therefore, the modeling exercise not only contributes to economic theorizing on discrimination, but also to social-psychological research in that it integrates social-psychological concepts in one well-defined formalized model.

The experiments described in chapter 4 focus on competition's effect on discriminatory behavior, without explicitly testing for the link via stereotypes and prejudice. The first two experiments also neglect financial costs, and hence the effect of increases in the marginal utility of profits. The third and fourth experiments include a 
2 The integrated framework

financial cost for making a decision, but only as a measure for the importance of e preference. They also do not analyse the 'economic effect of competition' as describen the theoretical model.

The business game (chapter 5) again abstracts from financial costs and margil utility of profits and takes expected productivity differences as the important 'econorr' factor, while focusing more on the influence of different stereotypes and prejudicesn discriminatory behavior.

Chapter 6 finally tests in how far efficiency-wage effects can play a role inn explanation of gender wage differences in the labor market, and in how far psychologil mechanisms apply also in very competitive (double-auction) settings. 


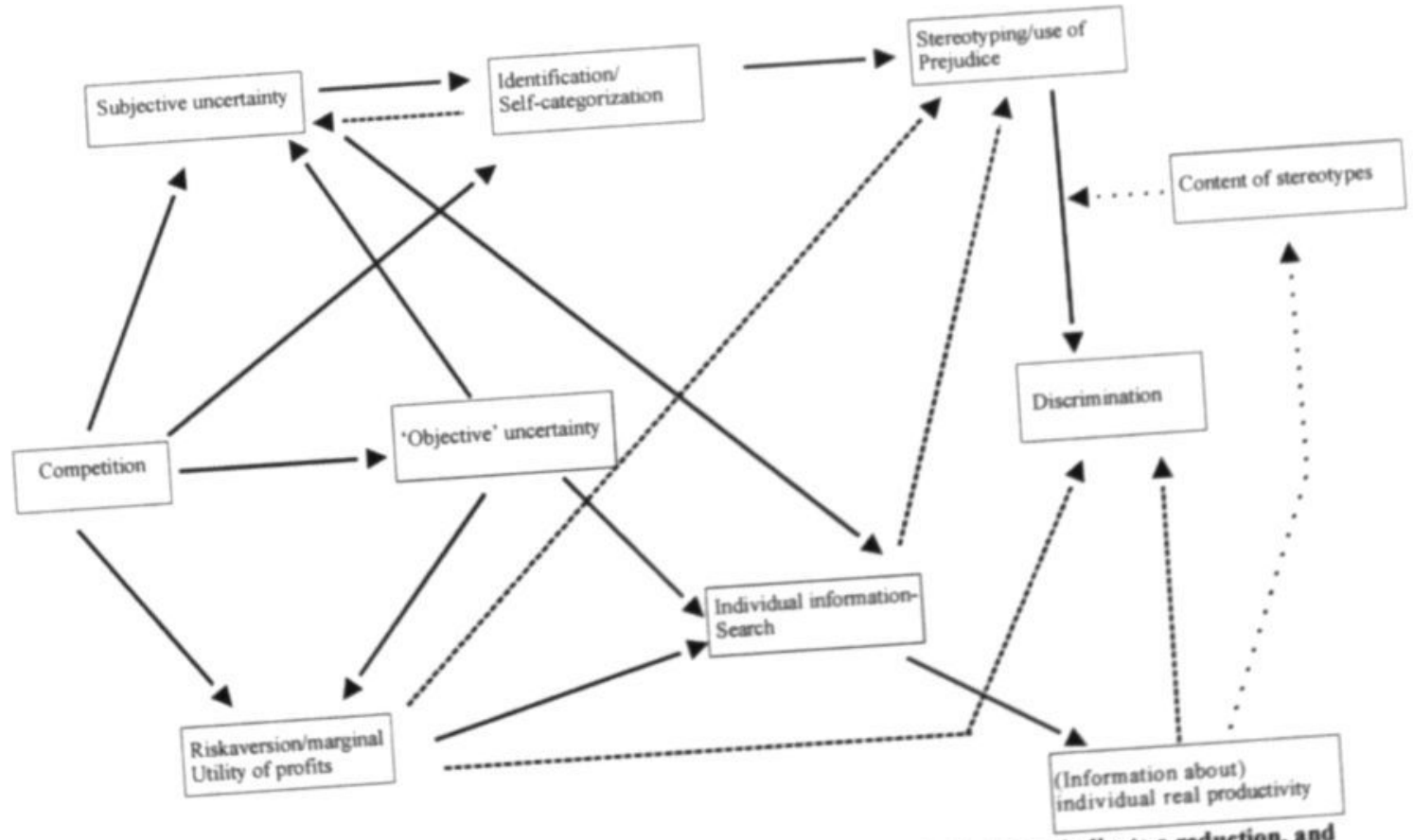

Figure 2.1: The general integrative framework - straight lines indicate 


\section{An Integrated model $^{26}$}

\subsection{Introduction}

In the previous chapter, economic and social-psychological literature on discrimination was reviewed. As was mentioned there, a striking difference between economic and social psychological approaches in general is that economic approaches usually treat variables like the extents to which stereotypes and prejudices lead to discriminatory behavior as given. Social-psychological models, on the other hand, distinguish between many different situations in which stereotypes and prejudices influence behavior to varying extents. The model developed here integrates this idea of social-psychological research into a microeconomic utility-analysis, endogenizing use of stereotypes and prejudice. These factors are used in social-psychological research for years to explain discriminatory behavior, but the insights gained from this research have so far been neglected by mainstream economic thinking to a high extent. On the other hand, standard economic theory offers a theoretical framework that is rigorous and well developed to analyze economic settings. As such, it is a good starting point for a more comprehensive analysis of discriminatory behavior in the labor market. Therefore, a formal utility model is developed, which explains the extent to which behavior in certain situations is guided by group perceptions, i.e. stereotypes and prejudice, within a microeconomic framework. Social identification is used as the mediating factor, which influences whether prejudice and stereotypes translate into behavior. Social identification, in turn, is influenced by situational factors, i.e., in this context, mainly the competitive situation of the firm in question. It is suggested that a strengthening of market competition may reinforce the use of stereotypes and prejudices, and that this, in its turn, may lead to more discrimination in the labor market. Indications from the 'real world' are that, e.g., in a country like Russia the old stereotype that men should have a job and women should stay at home has revived (Hunt, 1997), and at the same time labor market discrimination against women has increased.

An important purpose of the integration of social-psychological and economic theories is to construct a testable model. Our model is formulated such that it allows for

\footnotetext{
${ }^{26}$ This chapter is based on a working paper, written together with Maarten Vendrik: "Psychological vs. economic effects of competition on discrimination: An integrated model".
} 


\section{An Integrated model}

empirical testing with experiments and in field settings. First experiments have produced encouraging results, which will be reported in chapter 4 .

In the present chapter the social-psychological insights from chapter 2 are used to develop a model that tries to explain how an increase in competition can induce employers to rely more on stereotypic perceptions and prejudices in their hiring. pay and promotion decisions. The starting point of the model is the observation in social psychology that using stereotypic perceptions (for example, in personnel decisions) has certain benefits as well as costs (e.g., Fiske, 1998). The focus here is on the way in which an employer forms his perception of the relative productivities of a number of candidates for a position. He (or she) may base this perception on stereotypic information on the average productivity of groups candidates belong to (men/women, white/black, German/Dutch, etc.). However, he may also use individualized information from job interviews, hiring tests, etc. The extent to which he uses stereotypic perceptions versus individualized information in the formation of this perception then becomes an endogenous choice variable $(S)$. In choosing $S$ the employer is likely to maximize his expected utility of $S$, which reflects its benefits and costs.

One important psychological benefit of using stereotypes is the following: In a situation of high uncertainty about things which are important for the employer, like profits, (leading to self-relevant uncertainty in the terminology of Social Identity Theory (e.g., Mullin \& Hogg, 1998, see also sections 2.5 and 2.6.2), he is inclined to identify himself with a salient group he belongs to (his ingroup, e.g., men). In combination with using stereotypic perceptions this identification has the joint benefit of reducing feelings of uncertainty (i.e., the 'emotional' part of the self-relevant uncertainty, as opposed to the cognitive perception). Thus, using stereotypes and ingroup identification can be said to compensate for a lack of perceived self-relevant certainty in arousing feelings of (seeming) certainty. Hence, the model assumes that use of stereotypes and ingroup identification are substitutes for perceived self-relevant certainty in producing the basic commodity 'feeling certain' (Stigler \& Becker, 1977). Moreover, use of stereotypes and ingroup identification are assumed to be complements in producing this basic commodity.

A related psychological benefit of using stereotypes is that it may serve as a means to justify discriminatory behavior, and in particular to rationalize discriminatory tastes (see also Arrow, 1973). Whereas stereotypic perceptions are cognitive, prejudice represents a negative emotional evaluation, which may give rise to a discriminatory taste 
(Becker, 1957; Schaller et al., 1998). The extent to which prejudice is used in the formation of the discriminatory taste increases with ingroup identification. Hence, we assume that the use of prejudice is complementary to ingroup identification as well as use of stereotypes in producing feelings of certainty. In their turn, as a basic commodity, feelings of certainty are a determinant of the employer's expected utility.

Another basic commodity in the employer's expected-utility function is economic profits. The expected utility of these profits is affected by an economic cost of making use of stereotypes, which is the risk of mistakenly expecting an individual productivity difference between candidates from the ingroup and the outgroup at the expense of profits. The employer balances this cost against the benefits of making use of stereotypes and prejudice by maximizing his expected utility with respect to these variables as vell as ingroup identification (more or less consciously).

When competition increases, this affects the choice of stereotyping, using prejudice and identification in two ways. First, it raises perceived self-relevant uncertainty of the employer, which he will compensate by identifying more strongly with his ingroup and by relying more on stereotypic perceptions and prejudice in his perceptions and preferences with respect to candidates from the ingroup and the outgroup. This will lead to an increase in employer discrimination, which represents a psychological effect of strengthening competition on discrimination. On the other hand, by suppressing profits an increase in competition may also make employers more risk averse, implying a higher utility loss from the risk of mistakenly using stereotypic perceptions and prejudice. This will reduce the use of stereotypes, and hence lead to a decrease in employer discrimination. This economic effect of increasing competition on discrimination adds to the income effect of falling profits due to prejudice (Comanor, 1973) and counteracts the psychological effect explained above.

The stereotypical perceptions used may be correct as well as wrong. Socialpsychological research suggests that even wrong stereotypical perceptions can persist, among other things due to rationalization of prejudice and due to metacognitive bias in the perception of bigger ingroup samples versus smaller outgroup samples (Fiedler, 1996a, see also section 2.3.1).

This implies that the psychological effect of competition may raise discrimination even when there are no actual differences in productivity distributions, so even when discrimination is not rational from a profit-maximizing point of view. The counteracting risk aversion and income effects work in the same direction as the long-run selection 


\section{An Integrated model}

mechanism in Becker's theory of employer discrimination, according to which employers with zero discriminatory taste drive employers with a positive discriminatory taste out of the competitive product market. In this case, the psychological effect of competition may work in such a way that under competitive pressure even employers with zero discriminatory taste develop a positive one over time. This would then imply that not all employers with positive discriminatory taste would be driven out of the market.

Other economic theories (e.g. employee and customer discrimination, statistical discrimination, imperfect competition, self-fulfilling prophecies, gender differences in efficiency-wage effects; see Altonji and Blank (1999) for an overview and chapter 6 of this thesis on efficiency-wage effects) are able to explain that discrimination can be persistent under strengthening competition, but they seem not able to explain that discrimination may even increase under competitive pressure. Moreover, (almost) all economic theories seem to assume that the extent to which employers rely on stereotypic perceptions and prejudice does not change when competition increases. Both socialpsychological research and observations in countries like Russia suggest that reliance on stereotypes and prejudices may become stronger when competition intensifies, leading to an increase in discrimination.

A necessary condition for this to happen is that the situation at hand is seen as an intergroup situation, i.e., group-level categories must be salient. Paradoxically, this can be the case in hiring situations for predominantly ingroup occupations when outgroup members apply, especially when anti-discrimination laws exist, as this directs the focus towards groups rather than individuals. Flat hierarchies where teams are involved in hiring decisions can strengthen this perception of the situation as a group situation.

An integration of the social-psychological concepts into microeconomic theorizing can have other implications than those described above as well, and some special cases can lead to predictions very different from those of standard economic models. For example, anti-discrimination laws and policies promoting outgroups as equal or even, in some aspects, better than ingroups for a certain setting can lead to stronger perceived identity threat for the ingroup, and hence lead to the opposite result of what they aimed at.

The organization of the rest of the chapter is as follows. In Section 3.2 the basic assumptions are described, section 3.3 deals with the persistence of wrong stereotypes. The basic integrated model is developed in section 3.4 with respect to psychological 


\section{An Integrated model}

questions, the employer will in both cases get a larger sample of confirming answers for the hypothesis he is testing than for the contrary, even if the contrary is true, and he will thus conclude that the man is better than the woman (see Fiedler, Walther \& Nickel, 1999b). This approach helps to understand why stereotypes can be very persistent even if there is evidence to the contrary of the stereotype. This can happen without any motivational distortions, with (boundedly) rational information-processing agents, who just do not have the meta-knowledge about samples from the real world and how to interpret them with respect to making conclusions about the population.

Of course, there are other factors influencing the stereotypical perception $\triangle M P$ and the emotions correlated with it, e.g., socialization, the media etc. Emotional aspects are probably more difficult to change, as they partially work unconsciously, and thus can contribute to persistence of biased behavior and of stereotypes, when people try to justify their negative feelings about a certain group.

However, even when wrong stereotypic perceptions $\triangle M P$ tend to persist, the ameetion remains whv team members rely on such wrong stereotypes in their hiring decisions in the first place. Why do team members not use more reliable individual information on candidate team members from the ingroup and outgroup, e.g. from hiring tests, and why do they react group-based at all? These questions are addressed in the next sections.

\subsection{Basic model: psychological benefits}

\subsubsection{Hiring and pay preferences}

Consider a firm which consists of a team of $n$ team members. These team members receive equal profit shares in addition to a fixed base wage. Accordingly, they take democratic decisions about hiring a new team member from the ingroup or the outgroup. $^{30}$ Their preferences with respect to these decisions are implied by a utility function with respect to number of ingroup members $I$ and number of outgroup members $O$ in the team of the form ${ }^{31}$

$$
U(I, O ; \varepsilon)=U^{n}(\Pi n(I, O))-\varepsilon O, \quad U^{n}(\Pi n)>0, U^{\Pi n}(\Pi n)<0, \varepsilon>0
$$

\footnotetext{
${ }^{30}$ We abstract here from the question of the voting mechanism applied to come to a democratic decision by assuming homogeneous preferences and values of variables among all team members. This assumption implies that all team members will take identical decisions. The assumption can be relaxed, but is not likely to affect the outcomes of the model in an essential way.

${ }^{31}$ This represents a particular specification of a utility function proposed by Arrow (1973, pp. 6-7).
} 


\section{An Integrated model}

under the restriction $I+O=n$. Here $\varepsilon$ is a discriminatory-taste parameter and $\Pi n$ denotes each team member's expected profit share. This expected profit share is a function of $I$ and $O$ since team members rely on stereotypic perceptions of the average productivities of the ingroup and the outgroup in their expectations. This leads to an expectation $\triangle M P_{l}$ of a difference in productivity between an individual candidate team member from the ingroup and an individual candidate member from the outgroup. Hence, team members expect a difference in generated profits $\Pi$ equal to the output price $P$ times $\triangle M P_{t}$. This implies for each team member an average disutility of hiring an outgroup member instead of an ingroup member which is given by $U^{n_{1}}(n / n) P \overline{\Delta M P_{i}} / n$, where the average $\overline{\triangle M P}$ is taken over all possible pairs of candidates.

In addition, each team member expects a disutility $\varepsilon$ from hiring an outgroup instead of an ingroup member as a consequence of a prejudice against outgroup members. The total average disutility $D U$ of hiring an outgroup instead of an ingroup member is then given by

$$
D U=U^{n \cdot}(\Pi / n) P \overline{\Delta M P_{1}} / n+\varepsilon .
$$

This corresponds to the taste for discrimination as conceived by Becker $(1957 ; 1971)$ when he says that it incorporates both prejudice and ignorance about the absence of an actual (average) productivity difference between the discriminated and non-discriminated groups. However, since Arrow (1971) economists have reserved the term 'discriminatory taste' for the prejudice part $\varepsilon$ of $D U$ in eq. (3.1).

The discrimination coefficient of the team $D$, or the amount of profits the team is willing to sacrifice on average to avoid the disutility of hiring an outgroup instead of an ingroup member, is then equal to $n$ times $D U$ divided by $U^{n \cdot}(n / n)$, and so

$$
D=P \overline{\Delta M P_{i}}+n \varepsilon / U^{n_{1}}(\Pi / n) \text {. }
$$

\subsubsection{Formation of productivity perceptions $\triangle M P_{i}$}

How is expectation $\triangle M P_{l}$ of a difference in productivity between an individual candidate team member from the ingroup and an individual candidate member from the outgroup precisely formed? The team members base their expectation $\triangle M P_{\text {, partly on }}$ stereotypes since this saves on search for information on actual productivity differences, e.g. from individual hiring tests (which is also quite often ambiguous and requires effort to interpret it). The extent of using stereotypes $S$ in the formation of the expectation 
$\triangle M P_{i}$ is defined as follows. Assume that $S$ is continuously variable between 0 (no use of stereotypes) and $I$ (full use of stereotypes). Full use of stereotypes leads to a stereotypic perception $\triangle M P$, which, as explained in section 3.3 , can be substantially positive even in the absence of actual differences in average productivity. Less than full use of stereotypes $(S<I)$ means that expectation $\triangle M P_{i}$ is also based on information on actual differences in individual productivity for a part $1-S$. The latter information may be obtained from individual hiring tests, among other sources of individual information.

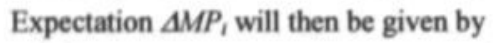

$$
\Delta M P_{1}=S \Delta M P+(I-S) \Delta M P_{i}^{T}=S\left(\Delta M P-\Delta M P_{i}^{T}\right)+\Delta M P_{i}^{T},
$$

where $\Delta M P^{T}$, denotes the productivity difference estimate from the hiring tests and other sources of individual information. ${ }^{32}$ This estimate will generally differ from zero, but assuming unbiased tests, the average estimate for a large number of candidate pairs will tend to zero. This implies that the average $\overline{\triangle M P}$ over a large number of candidate pairs tends to $S \triangle M P P^{33}$ Hence, the stereotype part of the discrimination coefficient $D$ in eq. (3.3) is given by $P$ times the stereotype-based part $S \Delta M P$ of expectations $\triangle M P_{i}$. Further assuming a symmetric ex ante distribution of $\Delta M P^{T}{ }_{1}$, it follows that the individual estimates $\triangle M P^{T}$, will mostly be lower than the stereotypic $\triangle M P$.

Use of stereotypes is also linked up, as described in section 3.1 , with identification with a group a person belongs to. The degree of identification with an ingroup $I$ can be interpreted as the degree to which the specific ingroup identity is salient as compared to the personal identity (see section 2.5.2). ${ }^{34}$ Therefore, we assume that (analogously to $S$ ) $I$ is continuously variable between 0 (zero weight of ingroup identity) and $l(100 \%$ weight of ingroup identity and zero weight of personal identity).

\footnotetext{
32 The variable $S$ corresponds to the coefficient $(l-y)$ of average ability $\alpha$ in eq. (3.2) of the statisticaldiscrimination model with performance tests of Aigner and Cain (1977). It is determined by the perceived relative reliability of $\triangle M P$ and $\Delta M P^{T}$, and hence by the amount of money, time and cognitive resources spent on collecting individualized information (e.g. by job interviews and hiring tests). We model this spending, and hence $S$, here as an endogenous choice variable. The hiring tests are only used for selection in hiring, but not as performance tests on which to base individual variations in wages since the hiring tests are supposed to be too unreliable for that purpose.

${ }^{33}$ Hence, note that the average of perceptions of individual productivity differences $\triangle M P$, is lower than the stereotypic perception of an average productivity difference $\triangle M P$ for $S<l$. Thus, the stereotypic perception is assumed to be insensitive to learning about individual productivity differences (see section 3.3). The model allows for cases where $\Delta M P$, and even $\triangle M P$ are negative.

${ }^{34}$ For the sake of simplicity, we assume that there is only one ingroup with which team members identify. This is a reasonable assumption at a certain point in time, in a certain setting, where one certain categorization is salient.
} 
Identification with an ingroup reduces feelings of uncertainty and also enhances a persons's self-esteem. Moreover, identification raises the use of stereotypes in the formation of the perception $\triangle M P_{1}$, which makes the world seem less uncertain. Conversely, a lower use of stereotypes seems only possible if the identification with the ingroup is reduced.

We model this functionality of relying on stereotypes and identification and their interactions as follows. The extent of using stereotypic perceptions $S$ and the degree of identification with an ingroup $I$ have the joint benefit of reducing feelings of uncertainty. Thus, $S$ and $I$ can be said to compensate for a lack of self-relevant certainty $C$ in arousing feelings of (seeming) certainty (and self-esteem) $\Psi$. Hence, $S$ and $I$ are assumed to be substitutes for $C$ in 'producing the basic commodity' $\Psi$ (Stigler and Becker, 1977). Hereby $C$ is conceived as the subjective perception by the team members of the probability that the team profits $\Pi$, and hence their profit shares $\Pi n$, will be nonnegative. Furthermore, since $S$ and $I$ seem to reinforce each other in arousing feelings of certainty $\Psi$, they are assumed to be complements in producing $\Psi$.

\subsubsection{Formation of discriminatory taste $\varepsilon$}

In many real-life situations stereotypes and prejudice go together (Kinder \& Sears, 1981). Whereas stereotypic perceptions are cognitive, prejudice represents a negative emotional evaluation. Psychological research also shows that the extent to which stereotypical perceptions and prejudices actually lead to discriminatory behaviour against an outgroup positively depends on the degree of identification with an ingroup (see section 2.5.2). Contrary to the equation of prejudice to the discriminatory taste $\varepsilon$ (see eq. (3.1)) in economic literature, we therefore make a distinction between prejudice and discriminatory taste, i.e. the disutility of hiring an outgroup member due to prejudice. Prejudice or negative emotional evaluation $E$ may be assumed to emerge as a psychological by-product of the stereotypical perception $\triangle M P$, but it may also represent a more general dislike as in the conception of Becker $(1957 ; 1971)$. Its intensity is likely to be positively correlated with conditioning variables like ingroup identification $I$ and amount and intensity of negative experiences (which are 'counted' into the value judgement), among other variables.

Discriminatory taste $\varepsilon$ can be assumed to be related to $E$, and hence to $I$ and other conditioning variables $Z$, as 


$$
\varepsilon=T E(I, Z), E_{l}>0, E_{Z}>0 .{ }^{35}
$$

Here $T$ is the extent to which prejudice is used in the formation of the discriminatory taste $\varepsilon$ (ranging from $\theta$ (no use) to $I$ (full use)). We consider $T$ as an endogenous choice variable. Since it increases with $I$ (see section 2.5.2), we assume that $T$ is complementary to $I$ in producing feelings of certainty $\Psi$.

There also seems to be an interaction of the use of prejudice $T$ with the use of stereotypes $S$ : A particular psychological benefit of $S$ is that it may serve as a means to justify discriminatory behavior and beliefs, in particular to rationalize discriminatory tastes (see also Arrow, 1973). We model this by assuming that $T$ is complementary to $S$ as well in producing feelings of certainty $\Psi$.

\subsubsection{Psychological benefits of S, I, T and C}

Use of stereotypes $S$, ingroup identification $I$, use of prejudice $T$ and perceived self-relevant certainty $C$ are assumed to be simultaneous 'choice' variables. This means each team member is supposed to maximize an expected (meta) utility $E \tilde{U}$ with respect to $S, I, T$ and $C$. This optimization may be more or less controlled or conscious. Especially with respect to $I$ and $C$ it will be uncontrolled and unconscious, but still it seems plausible to assume that $I$ and $C$ take on optimal equilibrium values (see below). Expected utility $E \tilde{U}$ is a function of two 'basic commodities': feelings of certainty $\boldsymbol{\Psi}$ and economic profit share $\Pi / n$. The latter is affected by perceived economic costs of $S, T$ and $C$, which will be analysed in the next sections. ${ }^{36}$ The utility function $E \widetilde{U}(\Psi, \Pi / n)$ is assumed to be separable in $\psi$ and $\Pi n$, i.e.

$$
E \tilde{U}=E \tilde{U}(\Psi, \Pi / n)=\tilde{U}^{\Psi}(\Psi)+E U^{\mathrm{n}}(\Pi / n),
$$

where $\tilde{U}^{\Psi}(\Psi)$ is not stochastic and has positive and diminishing marginal utilities with respect to $\Psi$. Certainty feelings $\Psi$ is given by a production function of $S, I, T$ and $C$

$$
\Psi=\Psi(S, I, T, C),
$$

\footnotetext{
3s A component of this discriminatory taste could be the disutility of a loss in ingroup identity of an ingroup member when he has to work with an outgroup member (see Akerlof and Kranton, 2000, p. 732). It is plausible that this component increases when the identification with the ingroup $I$ rises, as implied by eq. (3.5).

${ }^{36}$ Economic profits $\Pi$ are also affected by an economic benefit of using stereotypes $S$ due to economizing on financial resources, e.g. for individual hiring tests, but this is neglected here since it does not essentially change the model.
} 
with positive and diminishing marginal products except that $\Psi_{1}$ is zero for $I=I^{\bullet}$ between 0 and $I$ and negative for $I>I^{\bullet}$. Thus, we assume that it is not generally optimal in terms of feelings of certainty (and self-esteem) to identify fully with an ingroup at the expense of a zero weight of personal identity $(I=I)$. It seems more plausible to suppose that for a certain $I^{*}$ between 0 and $I$ there is an optimal balance between ingroup identity with weight $I^{*}$ and personal identity with weight $I-I^{*}$. This is modeled by $\Psi(S, I, T, C)$ reaching a maximum with respect to $I$ for $I=I^{*}$. Each team member is assumed to reach such a psychological equilibrium $I^{*}$ at which her certainty feelings (and self-esteem) are maximal with respect to $I$.

Perceived self-relevant certainty $C$ is also modelled as an endogenous 'choice' variable since social-psychological research shows that subjective perceptions of selfrelevant certainty are not fully determined by objective conditions (like fierceness of competition), but can be somewhat adjusted upwards by individuals in order to raise their feelings of certainty $\Psi$. On the other hand, limits to this form of self-deception are set by economic considerations (see the next section). Therefore, individuals also rely on $S, I$ and $T$ as substitute means to raise their feelings of certainty $\Psi$. The complementarity of $S, I$ and $T$ and their substitutability for $C$ in producing $\Psi$ are expressed by the following (in)equalities for the cross-second-order derivatives of production function (3.6):

$$
\Psi_{s}>0, \Psi_{s T}>0, \Psi_{\pi}>0, \Psi_{s c}=0, \Psi_{n c}=0, \Psi_{r c}=0 .
$$

Here the positive signs indicate Edgeworth complementarity of the variables involved in producing $\Psi$. For example, $\Psi_{S}>0$ means that a rise in ingroup identification $I$ leads to a rise in the marginal productivity $\Psi_{S}$ of using stereotypes $S$ in producing certainty feelings $\Psi$.

Substituting production function $(7)$ into $\tilde{U}^{\Psi}(\Psi)$ yields

$$
\tilde{U}^{\Psi}(\Psi)=\tilde{U}^{\Psi}(\Psi(S, I, T, C))=U^{\Psi}(S, I, T, C),
$$

with positive and diminishing marginal utilities with respect to $S, I, T$ and $C$ given by $U^{\Psi}=\tilde{U}^{\Psi} \Psi \Psi_{s}$, etc. This implies that the cross-second-order derivatives of utility function (3.9) are given by

$$
U^{\Psi} s=\tilde{U}^{\Psi} \Psi \Psi_{s}+\tilde{U}^{\Psi} \psi \Psi, \Psi_{s}, \text { etc. }
$$

Here the first term on the right-hand side is positive by virtue of the pertinent inequality in (3.8) and the second term is negative. We assume that the Edgeworth complementarity 


\section{An Integrated model}

of $S, I$ and $T$ in producing $\Psi$, as expressed by the inequalities in (3.8), is so strong as to make the first term dominant, and hence $U^{\Psi} s$ etc. positive. Thus, $S, I$ and $T$ are Edgeworth complements in producing utility $\tilde{U}^{\Psi}$ as well. ${ }^{37}$ For example, $U^{\boldsymbol{\psi}} s>0$ means that a rise in ingroup identification $I$ leads to a rise in the marginal utility $U^{\boldsymbol{\psi}} s$ of using stereotypes, which pushes the equilibrium $S^{*}$ (see the next section) to a higher value.

On the other hand, the equalities in (3.8) imply that $U^{\Psi} s \mathrm{e}$ etc. are negative as a result of the diminishing marginal utility of $\Psi$. Thus, $S, I$ and $T$ are Edgeworth substitutes of $C$ in producing $\tilde{U}^{\Psi}$. For example, $U_{\mathbb{C}}^{\boldsymbol{\psi}}<0$ means that a fall in perceived self-relevant certainty $C$ leads to a rise in the marginal utility $U_{i}^{\boldsymbol{\psi}}$ of identification, which pushes the equilibrium $I^{*}$ to a higher value. This property plays a key role in the effects of competition, which are elaborated in section 3.6. The underlying psychological mechanism by which the substitution is triggered is that a fall in $C$ leads to a fall in certainty feelings $\Psi$, which raises the marginal utility $\tilde{U}^{\Psi} \Psi$ of $\Psi$, i.e. fulfilment of $\Psi$ becomes more important relative to the profit-share motive $\Pi n n$ for the well-being of team members (see eq. (3.5)). ${ }^{38}$ By virtue of the relation $U^{\psi_{l}}=\tilde{U}^{\Psi} \Psi_{\Psi} \Psi_{1}$ this raises the marginal utility $U_{I}^{\Psi}$ of identification, leading to substitution of $I$ for $C$, and hence to a compensating rise in certainty feelings $\Psi$. We can now summarize the complementarity and substitutability relations found above as

$$
U_{S}^{\Psi}>0, U_{s T}^{\Psi}>0, U_{\pi}^{\Psi}>0, U_{s c}^{\Psi}<0, U_{\pi C}^{\Psi}<0, U_{\pi c}^{\Psi}<0 \text {. }
$$

If each team member's expected utility $E \tilde{U}$ were only given by the psychological utility benefits $\tilde{U}^{\Psi}(\Psi)$ in eq. (3.9), its maximization with respect to $S, I, T$ and $C$ would result in the maximal $S=I, T=I$ and $C=I$, i.e. full use of stereotypes and prejudice and maximal perceived certainty, and $I=I^{*}$, i.e. partial identification with the ingroup. By virtue of equation (3.4), the expected productivity difference $\Delta M P_{\text {, would then }}$ we

\footnotetext{
${ }^{37}$ The concepts of diminishing marginal utilities and Edgeworth complementarity in producing $\tilde{U}^{\boldsymbol{\psi}}$ presume that utility is cardinal. We make this assumption for at least three reasons. First, we need it anyway when we consider expected utility in the presence of risk in the next section. Secondly, it allows an easier and more intuitively appealing formalization of the psychological processes involving $S, I, T$, and $C$ than assuming ordinal utility. Thirdly, it allows us to give a cardinal meaning to expressions for the disutility $D U$ in eq. (3.2), and hence to consider the effects of competition on the size of $D U$ in section 3.6 . is This corresponds to the deprivation-domination principle in the needs theory of Maslow (1943), according to which the most deprived need is the most important in a person's motivation.
} 


\section{An Integrated model}

maximal and equal to the stereotypic $\triangle M P$. However, using stereotypes $(S)$ in the formation of $\triangle M P_{i}$ has also economic disadvantages for team members. This also holds for using prejudice $(T)$ in the formation of one's discriminatory taste $\varepsilon$ and having an unrealistically optimistic perception $(C)$ of self-relevant certainty. These costs are elaborated in the next session.

\subsection{Basic model: economic costs and equilibrium outcomes}

\subsubsection{Cost of $S$}

When using stereotypes, team members run the risk of mistakenly expecting a productivity difference $\triangle M P$, while the actual productivity difference is much smaller (on average zero). This would then go at the expense of the profits of the team, and hence of the members' profit shares. This risk is likely to be higher as more use is made of stereotypes in the formation of $\triangle M P_{i}$ since then less money, time and cognitive resources are spent on collecting individualized information in, e.g., hiring tests. The team members may know this more or less consciously and take it into account in the extent to which they rely on stereotypes in their perception $\triangle M P_{t}$. A measure for the perceived reliability of $\triangle M P_{l}$ is given by the standard deviation $\sigma_{i}$ of the subjective probability distribution $f\left(X_{i}\right)$ of the productivity difference $X_{i}$ of which $\triangle M P_{i}$ is the expected value $E X_{i}$ (after the perception $\triangle M P_{i}$ has been formed). In line with the arguments given above, this standard deviation $\sigma_{i}$ can be assumed to increase with $S$.

This assumption has implications for the expected utility $E \tilde{U}$ of each team member. This $E \tilde{U}$ does not only depend on psychological benefits $\psi$, but also on each team member's profit share $\Pi_{i} / n$. Here $i$ refers to the case where one of a particular pair of candidates from the ingroup and outgroup is hired. Suppose now that the expected

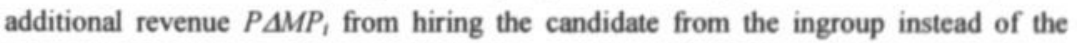
outgroup is higher than or equal to the additional payment $M D$ (market discrimination coefficient) for the ingroup candidate as determined in the labor market." The latter candidate will or may then be hired, and $\Pi_{4} / n$ is related to the stochastic productivity difference between the ingroup and outgroup candidates $X_{i}$ as $\Pi_{i} / n=\left(\Pi+P X_{i}-M D\right) / n$.

\footnotetext{
"Additional revenue $P \Delta M P$, can be higher than $M D$ due to a positive $\Delta M P^{T}$, from individual information or when productivity differences between the particular pair of candidates are perceived differently by different teams (cf. the assumption of heterogeneous discriminatory tastes in the employer-discrimination model of Becker, 1957, 1971). An analogous analysis with the same conclusions can be given for the case where $P \triangle M P$, is lower than or equal to $M D$ and the outgroup candidate is hired.
} 


\section{An Integrated model}

Here $\Pi$ is team profits in the absence of differences in productivity and payment between the ingroup and outgroup candidates. To derive a tractable expression for $E U^{n}\left(\Pi_{i} / n\right)$, we approximate $U^{n}\left(\Pi_{i} / n\right)$ by its second-order Taylor expansion with respect to $X_{i}$ around its expected value $\triangle M P_{i}$ (as usual in risk analysis). This Taylor expansion is given by

$U^{n}\left(\Pi_{i} / n\right)=U^{n}\left(E \Pi_{i} / n\right)+\partial U^{n} / \partial X_{i}\left(\Delta M P_{j}\right)\left(X_{i}-\Delta M P_{i}\right)+1 / 2 \partial^{2} U^{n /} / \partial X_{i}^{2}\left(\Delta M P_{j}\right)\left(X_{i}-\Delta M P_{i}\right)$.

Applying the chain rule to the first and second-order derivatives and using $E X_{i}=\Delta M P_{i}$ and $E\left[\left(X_{i}-\Delta M P_{i}\right)^{2}\right]=\sigma_{i}^{2}$, we obtain

$$
E U^{n}\left(\Pi_{1} / n\right)=U^{n}\left(E \Pi_{1} / n\right)+1 / 2 U^{n}\left(E \Pi_{1} / n\right) P^{2} \sigma_{i}^{2} / n^{2} \text {. }
$$

Since $U^{n} n\left(E \Pi_{1} / n\right)$ is negative for the assumedly risk-averse team members, the last term in this equation represents the expected utility loss due to the perceived risk that the actual productivity difference between the ingroup and outgroup candidates will turn out to be lower than expected on the basis of stereotyping and individual-specific information. This loss is higher as the standard deviation $\sigma_{i}$ of the subjective probability distribution of the productivity differences between the ingroup and outgroup candidates is greater. Hence, since it is plausible to assume that more use of stereotypes (a higher $S$ ) leads to a greater expected $\sigma_{i}$, the utility loss will be greater as $S$ is higher.

Suppose, for the sake of simplicity, that $\sigma_{i}$ is linear in $S$ :

$$
\sigma_{i}=\sigma^{0}+\sigma^{l} S, \quad \sigma^{0}>0, \quad \sigma^{l}>0 .
$$

Moreover, we can approximate $U^{n}\left(E \Pi_{i} / n\right)=U^{n}\left[\left(\Pi+P \Delta M P_{r}-M D\right) / n\right]$ by $U^{n}(\Pi n)$ and $U^{n \prime}(E \Pi / n)$ by $U^{n n}(\Pi n) .^{40}$ Substituting these approximations and eq. (3.14) into eq. (3.13), it then follows that the utility loss in eq. (3.13) is quadratic in $S$.

\subsubsection{Costs of $T$ and $C$}

Using prejudice $(T)$ in the formation of one's discriminatory taste $\varepsilon$ is not costless since team members have to pay a money price to indulge their discriminatory taste. This price is given by the market discrimination coefficient $M D$ and will be paid if and only if it is advantageous to hire an ingroup member, i.e. if and only if the team's discrimination coefficient as given by eq. (3.3) with $\triangle M P_{t}$ instead of $\overline{\triangle M P_{i}}$ is greater than or equal to

\footnotetext{
${ }^{40}$ These approximations and assumption (3.13) are not essential for the main implications of the model. The approximation of $U^{n}\left(E \Pi_{1} n\right)$ by $U^{n}(n n)$ eliminates a perverse incentive to use more stereotypes from the model. This incentive results from the fact that a higher $S$ mostly leads to a higher expected $\Delta M P_{b}$, and hence to a higher expected profit share $(\Pi+P \Delta M P,-M D) n$. However, this represents a form of selfdeception which is likely to be corrected to a certain extent when team members are aware of it.
} 


\section{An Integrated model}

MD. Substituting eq. (3.5) into eq. (3.3) it then follows that the cost $M D$ is incurred on average if and only if

$$
T \geq U^{\mathrm{n} \cdot(\mathrm{I} / n)(M D-P S \Delta M P) /(n E(I, Z)) .}
$$

Thus, for $T$ satisfying this inequality there is a utility cost of $T$ given by $U^{\mathrm{n} \cdot}(\mathrm{\Pi} / n) M D$. We assume that team members take this utility cost into account (more or less consciously) in 'choosing' their optimal extent $T$ of using prejudice in the formation of $\varepsilon$.

Having an unrealistically optimistic perception $(C)$ of self-relevant certainty has also a cost from a higher risk of overestimation of the probability that the profit share $\Pi / n$ will be non-negative ('risk of having the wrong belief, see Camerer and Weber, 1992, p. 326). This overestimation may be due to an unrealistically high expected value of sold output $Q$ or of an unrealistically low subjectively perceived variance of $Q$. Team members are assumed to be vaguely aware of these possible biases, leading to a higher perceived variance of their subjective probability distribution of the expected value or variance of $Q$ (a second-order probability distribution in the terminology of Camerer and Weber (1992), see section 2.5). For the risk-averse team members this higher perceived (second-order) risk leads, analogously to the economic cost of $S$, to an expected loss in utility of profits. This utility loss is not formalized here, but will be higher as perceived self-relevant certainty $C$ is (unrealistically) higher.

Although insights from social-psychological research suggest that a fall in $C$ is compensated for by rises in $I, S$ and $T$ in arousing certainty feelings $\Psi$ (see section 3.4.4), they also suggest that there is a reinforcing feedback from the rises in $I, S$ and $T$ towards a higher perceived self-relevant certainty $C$ (due to a more biased view of the world; see, e.g., Mullin \& Hogg, 1998). Such a feedback can be modeled by assuming that rising $I, S$ and $T$ suppress the awareness of team members of possible biases in their perception of self-relevant certainty, and hence lower the expected utility loss of a higher $C$.

\subsubsection{Equilibrium outcomes}

The team members are supposed to maximize $E \tilde{U}$ as given by eq. (3.6). By virtue of eqs. (3.9) and (3.14) and the analysis in the previous subsection, this can be written as a function of $S, I, T$ and $C$ alone: 


\section{An Integrated model}

$$
E \tilde{U}=U^{\Psi}(S, I, T, C)+\left(E U^{\mathrm{n}}\right)(S, T, C)^{41}
$$

Since the economic-cost part $-\left(E U^{n}\right)(S, T, C)$ of $E \tilde{U}$ is discontinuous with respect to $T$ at the value of $T$ given by the right-hand side of eq. (3.15), we do not have a first-order condition with respect to $T$. However, plotting benefits $U^{\Psi}(S, I, T, C)$ and the utility cost of $T$ as functions of $T$, it is easily seen that there are two local maxima of $E \tilde{U}$, namely at the value of $T$ in eq. (3.15) and at $T=1$. Which of these local maxima is the global maximum, depends on, among other things, the level $U^{n !}(\Pi / n) M D$ of the utility cost of $T$. When this level is sufficiently high, the lower value of $T$ is the globalmaximum point $T^{*}$. The team members then do not fully use their prejudice against outgroup members, and hence do not hire ingroup members, because of the high economic cost involved.

However, the equilibrium value $T^{*}$ is simultaneously determined with the equilibrium values $S^{*}, I^{*}$ and $C^{*}$ of $S, I$ and $C$ via the complementarity and substitutability relations (3.11) and via eq. (3.15). With respect to $S, I$ and $C$ the following first-order conditions for maximization of $E \tilde{U}$ can be formulated:

$$
\begin{aligned}
& U_{S}^{\psi}(S, I, T, C)+\left(E U^{\mathrm{n}}\right)_{S}(S, T, C)=0 \\
& U_{I}^{\psi}(S, I, T, C)=0 \\
& U_{C}^{\psi}(S, I, T, C)+\left(E U^{\mathrm{n}}\right)_{C}(S, T, C)=0
\end{aligned}
$$

Condition (3.17b) leads to $I=I^{*}$ (see analysis under eq. (3.7)). Condition (3.17a) can also be written as

$$
U_{S}^{\Psi}(S, I, T, C)=-\left(E U^{\mathrm{n}}\right)_{S}(S, T, C) .
$$

The left-hand side of this equation gives the marginal benefits $M B$ for each team member of more use of stereotypes in the selection of a new team member, whereas the right-hand side represents the marginal cost $M C$ of more use of stereotypes due to higher risk for profit shares. Diminishing marginal utility of using stereotypes (condition under eq. (3.9)) implies that $M B$ falls as a function of $S$. On the other hand, $M C$ is obtained by applying the chain rule to eqs. (3.13) and $(3.14)^{42}$, yielding

$$
M C:=-\left(E U^{n}\right)_{s}(S, T, C)=-U^{n n}(\Pi / n) P^{2} \sigma^{l}\left(\sigma^{0}+\sigma^{l} S\right) / n^{2} .
$$

\footnotetext{
${ }^{41}$ We abstract from the dependence of $\left(E U^{\mathrm{n}}\right)$ on $I$ via eq. (3.15).

${ }^{42}$ We abstract from the dependence of $\left(E U^{\mathrm{n}}\right)$ on $S$ via eq. (3.15).
} 
Since $U^{n}(\Pi / n)$ is negative, this equation implies that $M C$ is positively linear in $S$. Both $M B$ and $M C$ as functions of $S$ for given $I, T$ and $C$ are drawn in Fig. 1. Utility is maximized at $S^{*}$, where $M B=M C$. By virtue of eq. (3.4), this implies an equilibrium value $\Delta M P_{1}{ }^{*}$ of expectation $\Delta M P_{1}$ of a difference in productivity between particular candidates from the ingroup and the outgroup. The ensuing stereotype part of the discrimination coefficient $D^{*}$ (see eq. (3.3)) is then given by $P$ times the stereotypebased part $S^{*} \triangle M P$ of expectations $\triangle M P_{1}$. 


\section{An Integrated model}

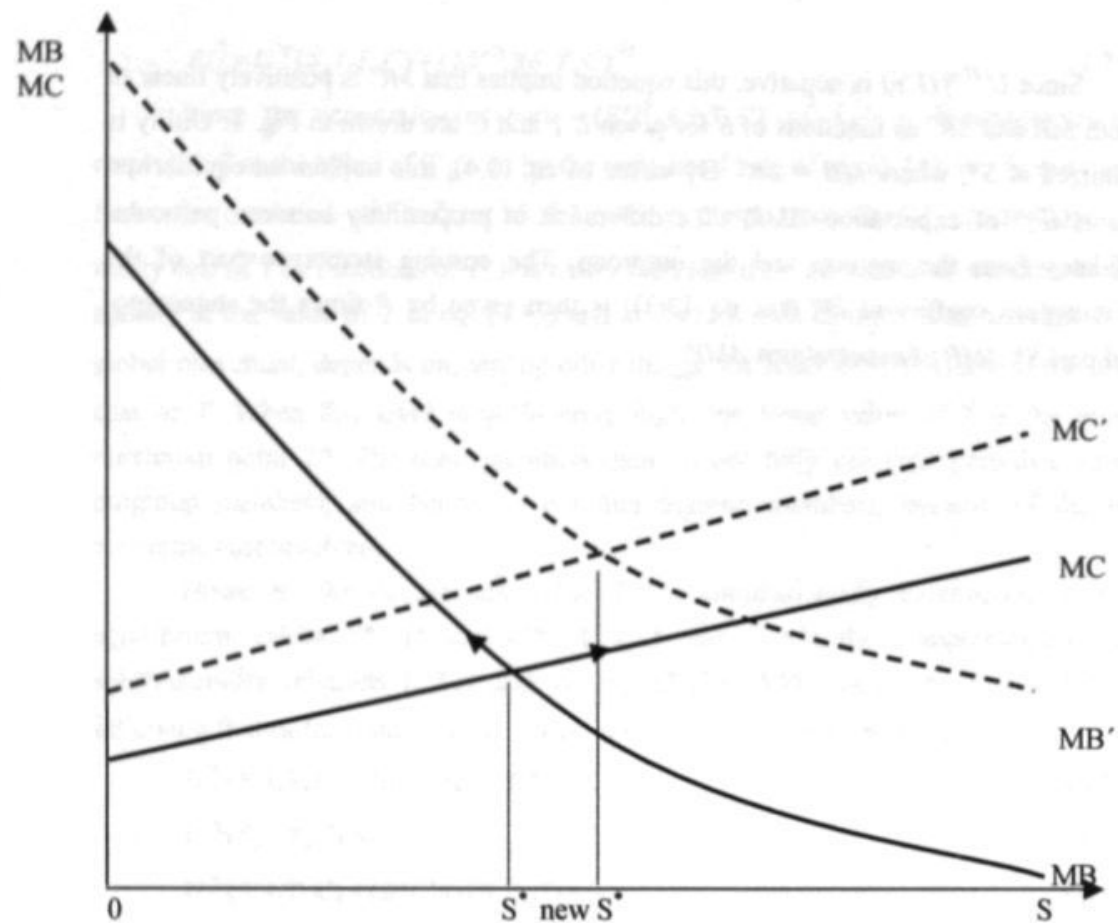

Figure 3.1: Change in the equilibrium value of the extent of using stereotypes $S$ due to an increase in competition in the production market.

However, the equilibrium value $S^{*}$ is simultaneously determined with, and hence conditional on, the equilibrium values $I^{*}, T^{*}$ and $C^{*}$. The equilibrium value $C^{*}$ is determined, analogously to eq. (3.18), by equality of marginal benefits and marginal costs of perceived self-relevant certainty $C$. This leads to a graph similar to fig. 1 , but now there is also feedback from rising $S, I$ and $T$, which lowers the marginal cost curve, and hence leads to a higher $C^{*}$ (see the previous subsection).

The equilibrium values $T^{*}$ and $I^{*}$ imply, via eq. (3.5), an equilibrium value $\varepsilon^{*}$ of the discriminatory taste of each individual team member (assumed to be homogeneous). This determines the prejudice part $D_{c} *$ of the discrimination coefficient $D^{*}$ according to eq. (3.3) for $\varepsilon=\varepsilon^{*}$. Substituting eq. (3.4) for $T=T^{*}$ and $I=I^{*}$ into this equation, the total discrimination coefficient $D^{*}$ is then given by

$$
D^{*}=D_{S * a M P}+D_{e}=P S^{*} \Delta M P+n T^{*} E\left(I^{*}, Z\right) / U^{n}(\Pi n) .
$$


The empirical finding that prejudice is a better predictor of discriminatory behaviour than stereotypes are (e.g., Stangor et al., 1991; Dovidio et al., 1996) suggests that prejudice is more difficult to suppress, and hence that $D_{e}$ can be large relative to $D_{s \cdot \Delta u r .}$

On the other hand, there are also indications that people try to justify or rationalize their negative 'gut feelings' about a certain group by more stereotyping (see sections 2.3.1 and 3.4.3). In the present context this may mean that the discriminatory taste $\varepsilon^{*}$ is fully or partially rationalized into a higher $S^{*} \Delta M P$. Whereas a prejudice $E$ can contribute to the persistence of a wrong stereotypical perception $\triangle M P$ (see section 2.3.1), the use of prejudice $T^{*}$ may raise the use $S^{*}$ of such a stereotypical perception rather than enlarge the stereotypical perception itself. This is modelled by the interaction of $T$ and $S$ in utility function (3.9) as expressed by the positive sign of its second-order derivative $U_{s}^{\psi}$ according to inequalities (3.11). This raises the marginal utility of using stereotypes $U_{s}^{\nabla}$, i.e. the marginal benefits $M B$ of $S$, implying an upward movement of the $M B$ curve in Fig. 1. This leads to a rise $\Delta S^{*}$ in the equilibrium value $S^{*}$, and hence to an

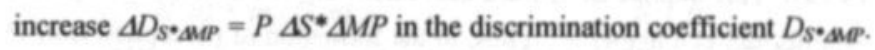

The increase $\Delta D_{S \cdot M P}$ can be expected to be lower than or equal to the discrimination coefficient $D_{e}$ due to $\varepsilon^{*}$, i.e. $\Delta D_{s * M P}=\alpha D_{e}$ with $0<\alpha \leq 1$. The cases $\alpha<l$ and $\alpha=I$ may then be said to correspond to partial and full rationalization, respectively, of the discriminatory taste $\varepsilon^{*}$ as an increase $\Delta S^{*}$ in the use of stereotypes. Now it seems plausible to assume (as a first approximation) that neither partial nor full rationalization affects the value of the total discrimination coefficient $D^{*}$ in eq. (3.20). This implies that after rationalization

$$
D^{*}=D_{s}^{*} \Delta M_{P}+(I-\alpha) D_{e}=P S^{*} \Delta M P+(I-\alpha) n T^{*} E\left(I^{*}, Z\right) / U^{n}(I n n) .
$$

Thus, in the case of full rationalization $(I-\alpha=0)$ no separate contribution of $\varepsilon^{*}$ to $D^{*}$ is left, whereas the case of partial rationalization $(I-\alpha>0)$ still allows some separate contribution of $\varepsilon^{*}$ (which may work unconsciously).

The corresponding formula for each team member's total average disutility $D U$ of hiring an outgroup instead of an ingroup member (eq. (3.2)) then becomes

$$
D U^{*}=D U_{s * \Delta M P}+(I-\infty) D U_{e}=U^{n}(I n n) P S^{*} \Delta M P / n+(l-\infty) T^{*} E\left(I^{*}, Z\right) .
$$




\subsection{Effects of competition}

What happens with the discrimination coefficient $D^{*}$ and the disutility $D U^{*}$ when competition intensifies, i.e. when team members' average profit shares $\Pi / n$ fall? On the one hand, there is a psychological effect due to higher self-relevant uncertainty (see section 2.5 .2 ). Sticking to the 'old' perception $C$ of a relatively high probability that profit shares will be non-negative then bears an increasing risk of overestimation. This raises the perceived marginal $\operatorname{costs}$ of $C$, and hence leads to a lower equilibrium value $C^{*}$ of perceived self-relevant certainty $C$. By virtue of the negative signs of the second-order derivatives $U_{\pi C}^{\Psi}, U_{s c}^{\Psi}$ and $U_{\pi C}^{\Psi}$ according to inequalities (3.11), the marginal utilities of ingroup identification $I$, using stereotypes $S$, and using prejudices $T$ then rise. This pushes $I, S$, and possibly $T$ towards higher equilibrium values, i.e. the lower perceived selfrelevant certainty is substituted (or compensated for) by stronger ingroup identification and use of stereotypes (and prejudice) in order to arouse sufficient feelings of certainty $\Psi$. In particular, the lower perceived self-relevant certainty raises the marginal benefits $M B$ for each team member of more use of stereotypes in the selection of a new team member (see eq. (3.18)). This implies an upward movement of the $M B$ curve in fig. 3.1, and hence a new equilibrium at a higher $S^{*}$. Thus, in the formation of perception $\triangle M P_{i}^{*}$ more use is made of stereotypes, leading to a higher $\triangle M P_{i}^{*}$.

This effect is reinforced if the stronger group identification $I^{*}$ also enlarges the stereotypical perception $\triangle M P$ itself. On the other hand, the rises in $I, S$ and possibly $T$ feed back to a higher perceived self-relevant certainty $C$ (see section 3.5 .2 ), which somewhat suppresses the rises in $I^{*}, S^{*}$ and $T^{*}$. Furthermore, the appendix shows that, under plausible conditions, maximization of the team members' expected (utilities of) profit shares implies that under increasing competition the number of team members $n$ may rise, and hence the expected marginal team productivity of the last team member MP may fall. The expected difference in average marginal team productivity between last ingroup and outgroup members $\triangle M P$ may then fall accordingly (consider e.g. a Cobb-Douglas production function with decreasing returns to scale ${ }^{43}$ ). The total effect of increasing competition on $\triangle M P$ then is ambiguous. Finally, in the expression for the discrimination coefficient $D_{S} \cdot \Delta M P=P S^{*} \Delta M P$ increasing competition implies a fall in $P$. The overall effect of increasing competition on $D_{S} \cdot \Delta M P$, and hence on the total

\footnotetext{
${ }^{43}$ With decreasing returns to scale there exists a unique profit-maximizing level of output.
} 
discrimination coefficient $D^{*}$ (see eq. (3.21)), may then still be positive, but this is uncertain. In addition, when there is a discriminatory taste $\varepsilon^{*}$ which is only partially rationalized as a higher $S^{*}$, rises in $n, T^{*}$ and $E\left(I^{*}, \mathrm{Z}\right)$ due to the rise in $I^{*}$ raise the discrimination coefficient $D_{\infty}$, and hence the total discrimination coefficient $D^{*}$, according to eq. (3.21). Thus, most of the positive effects on $D^{*}$ explained above are a consequence of the stronger and higher-valued identification with the ingroup as a means to reduce the higher perceived self-relevant uncertainty due to stronger competition. ${ }^{44}$

On the other hand, when team members' profit shares $\Pi n$ fall there is also an economic effect due to changes in $-U^{n} \eta(\Pi / n) P^{2} / n^{2}$ in expression (3.19) for the marginal costs of stereotyping $M C$. Minus the second-order derivative $U^{\Pi m}(\Pi / n)$ can be given an economic interpretation by rewriting it as

$-U^{n}(n / n)=\left(-U^{n}(\eta / n) / U^{n}(\eta / n)\right) U^{n}(\Pi / n)=r(\Pi n) U^{n}(\Pi / n)$,

where $r(\Pi n)$ is Pratt's measure of risk aversion. It is well-known that when we approximate the utility function $U^{n}(\Pi / n)$ by the power function

$$
\begin{aligned}
U^{n}(\Pi / n) & =(\Pi / n)^{R} / R, & R<1, R & \neq 0, \\
U^{n}(\Pi / n) & =\ln (\Pi / n), & R & =0,
\end{aligned}
$$

$r(\Pi n)$ rises as the profit shares $\Pi n$ fall (Nicholson, 1998, pp. 225-226). This means that team members become more risk averse as their profit shares fall, which is intuitively plausible. In addition, the marginal utility of profit share $U^{n}(n / n)$ also rises as $\Pi n$ falls. On the other hand, these rises are counteracted by the fall in $P^{2}$ in expression (3.17) for $M C$ when competition intensifies. The latter effect holds since the fall in $P$ directly lowers the expected difference in revenues $P \Delta M P_{i}^{*}$ from an ingroup versus an outgroup member (i.e., less is at stake in money terms).

To see which of the last two effects may be more likely to dominate, we again approximate the utility function $U^{n}(\Pi / n)$ by the power function (3.24). This implies $U$ ${ }^{\prime}(\Pi / n)=(\Pi / n)^{R-1}$ for all $R<I$. Pratt's measure of relative risk aversion is then equal to the constant $I-R$. Empirical evidence is generally consistent with values of $R$ in the range of -3 to -1 , and it is easily shown that for $R \leq 0$ a fall in $P$ and the ensuing fall in $\Pi$ together lead to a net rise in

\footnotetext{
${ }^{4}$ In addition, the use of stereotypes $S^{*}$, and hence $D^{\bullet}$, may rise with increasing competition since the disutility of financial costs $c$ of individual hiring tests, which is given by $U^{n}(\eta / n) c$, rises.
} 
$-U^{n}(\Pi / n) P^{2} / n^{2}$ in expression (3.19) for $M C$ (see appendix for a derivation). This implies that if team members are not much less risk averse than generally measured, the marginal cost of stereotyping $M C$ rises as competition increases. This results in an upward movement of the $M C$ line in fig. 3.1, and hence a new equilibrium at a lower $S^{\bullet}$. This would lead to a lower perception $\Delta M P_{i} *$ as well as a lower discrimination coefficient $D_{S^{*} M P}=P S^{*} \Delta M P$ provided $\triangle M P$ does not rise too much (see above). In addition, when there is a discriminatory taste $\varepsilon^{*}$ which is only partially rationalized as a higher $S^{*}$, the rise in $U^{n}(\Pi / n)$ suppresses the discrimination coefficient $D_{\varepsilon}$, and hence the total discrimination coefficient $D^{*}$, by virtue of eq. (3.21). This represents an income effect of falling profits (Comanor, 1973), according to which falling profits make it relatively more expensive to indulge one's discriminatory taste, and hence suppress the amount of money one is willing to spend on it. The rise in $U^{\mathrm{n} \cdot}(\Pi / n)$ may also suppress $D_{e}$ in an indirect way by pushing the use of prejudice $T^{*}$ from full to partial (as given by the right-hand side of eq. (3.15)) due to a rise in the utility cost $U^{\mathrm{n} \cdot}(\Pi / n) M D$ of $T$.

The signs of the net changes in $S^{*}$ and $D^{*}$ due to the combination of psychological and economic effects then are ambiguous, and should be measured in experiments or empirical research. On the other hand, the net changes in each team member's total average disutility $D U$ of hiring an outgroup instead of an ingroup member (eq. (3.22)) seem somewhat less ambiguous since they differ from the team's discrimination coefficient $D^{*}$ by a factor $U^{n}(\Pi / n) / n$, and especially the rise in $U^{n}(\Pi)$ $(n)$ may tend to render the net changes in $D U$ positive. The factor $U^{n}(\Pi / n)$ in eq. (3.22) can be interpreted as the subjective importance of perceived differences in profit shares in the hiring preferences of each team member. When competition intensifies, this importance rises and thus more than compensates the suppressing effect of a falling output price $P$ on $D U$ under the assumptions made above. 


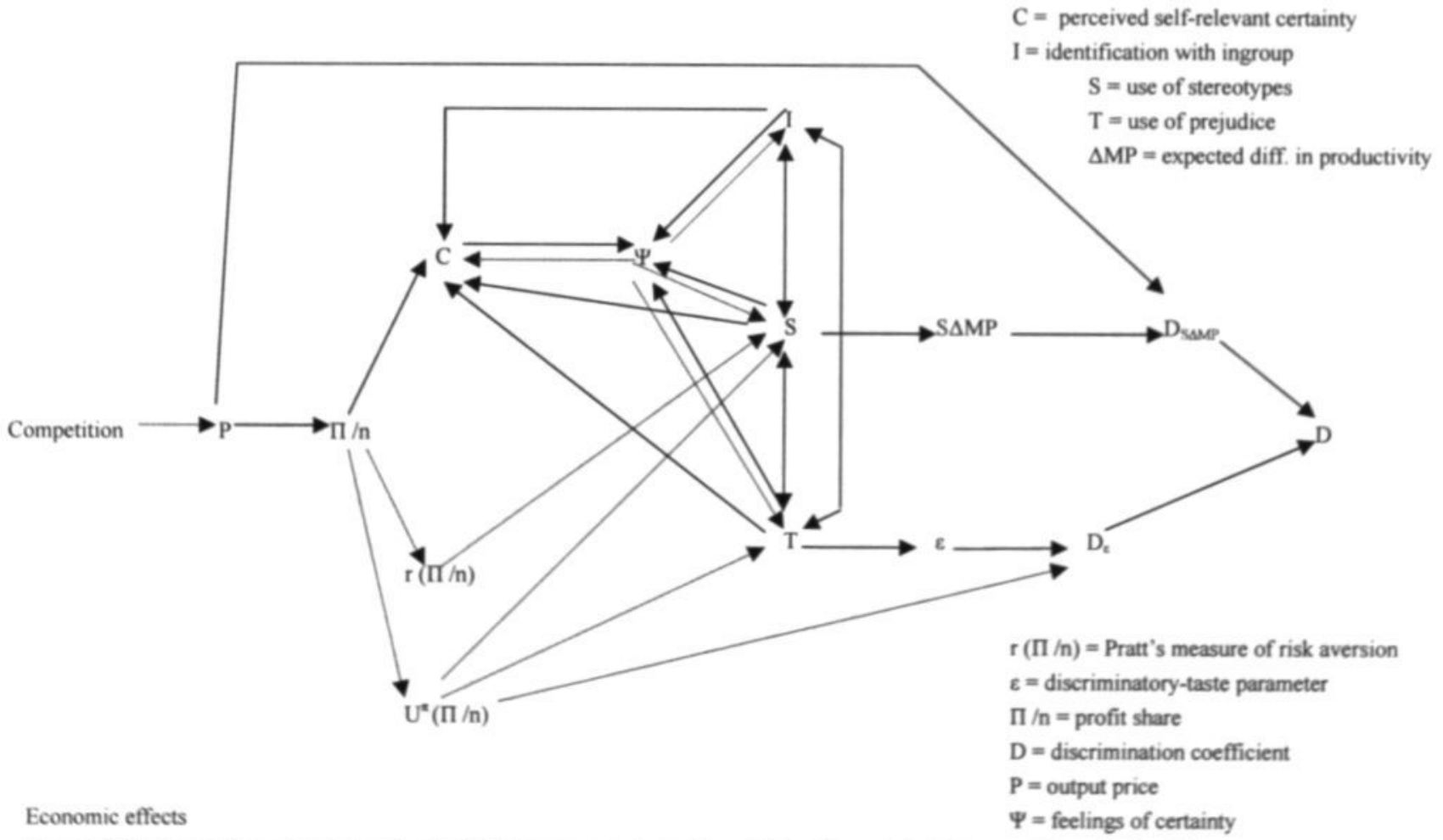

Figure 3.2: The relationships assumed - dotted lines represent negative relationships, straight lines positive relationships 


\subsection{Concluding remarks}

In this chapter an integrated model of discrimination in the labor maket was developed. The model aims at explaining the persistence and possible strengtining of discrimination in the labor market in spite or even because of competitive ressure, making use of psychological concepts. Using the assumption of firms being strutured in teams, it looks at employer as well as employee discrimination. Furthermore, w wanted to create an empirically testable model, and a model on the basis of which well-1formed policy recommendations can be made. In this last section we discuss in hov far the model proposed reaches these aims.

Endogenizing the use of stereotypes, prejudice and identification, the mdel can explain how in certain situations discrimination can persist or even get strongr when competition strengthenes. However, it also leaves some important aspects open On one hand, it shows that in cases where the psychological effect is stronger, discrimintion can persist or get stronger when competition gets stronger. On the other hand, $\mathrm{i}$ is also possible that the economic effect is strogeer and.tban diersimination wri" hor persist. This shows, that the predictions of the model are still ambiguous, and it is not yet clear under what conditions which effect will be stronger.

A second open question pertains to what happens when, e.g., the stereotype of the outgroup in some aspects, which are relevant for the work situation, is positive, and these aspects are made salient. For example, a strong case could be made - as it currently is for the importance of interpersonal skills for the success of a firm, and these skills are stereotypically stronger in women. Would in that case a more stereotypic perception of the outgroup lead to more hiring of women? Or do men see this as a threat, which leads to stronger identification and stronger use of prejudice against women? Or will men make other aspects of the female stereotype salient, which point again to a negative evaluation of women?

More from a psychological point of view, the question remains how the interplay between the cognitive and emotional factors works, and what happens, when they are conflicting, e.g., when there is prejudice against a group, but cognitive evaluations of this group are clearly favorable.

Rigorous empirical testing, in experiments as well as in field studies can probably give answers to the first two questions. Basically, the model allows for this kind of testing. However, a problem of the model for experimental testing is that in some aspects 
it might be over-determined - cognitive and emotional factors can lead to discriminatory outcomes, when there is enough identification - and they all can lead to discriminatory outcomes on their own, following psychological theory. Moreover, the situation in the real world is over-determined as well, in the sense that with 'real-life-categories' many more facets of the stereotypes and the history of group relations are linked, which make the situation much more complex. In future research, it could be fruitful to extend the model such as to include specific contents of stereotypes.

The theoretical model shows the possibility of an interaction of economic and psychological effects of strengthening competition. The results of the experiments described in the next chapters can help to answer the question when one or the other of the partial effects gets stronger.

\section{Appendix: Derivations of effects of competition}

Once team members have 'chosen' their optimal extents of using stereotypes $S$, identification $I$, and using prejudice $T$, their choice of the optimal number of team members $n$ is determined by maximization of their expected utility of profit share. Suppose first that all team members belong to the ingroup. In that case their discriminatory tastes do not play a role, implying that the team members maximize their expected profit share. The number of team members $n$ then is the highest integer that is lower than the real number $\tilde{n}$ such that, in continuous approximation, $P M P=W+\Pi \tilde{n}$, where both the marginal team productivity $M P$ and profits $\Pi$ are functions of $\hbar$ and where $W$ is the equilibrium base wage for ingroup members. This means that the additional revenues from the last team member have to cover his base wage as well as the prevailing profit share. Profits $\Pi$ are equal to $P Q-W \tilde{n}-K$, where $Q$ is output quantity and $K$ is non-wage costs. The above condition for profit-share maximization can then be rewritten as $M P=Q / \tilde{n}-K /(P \tilde{n})$. Hence, $\tilde{n}$ is determined by the intersection of a downward-sloping $M P$ curve (decreasing returns to scale) and a curve representing the right-hand side of this equation. Moreover, when competition becomes stronger, c.p., and hence the output price $P$ falls, the latter curve moves downward. This implies a higher $\tilde{n}$ as well as a lower MP. However, when the non-wage costs $K$ are sufficiently low and/or the marginal team productivity $M P$ falls sufficiently steeply for increasing $\tilde{n}$, the integer number of team members $n$ will not change within a certain range of variation in competition. There will then be no downward effect on MP, and hence on $\triangle M P$. Under 
3 An Integrated model

plausible conditions, these results can also be shown to hold when some or more of the team members are outgroup members.

Another result mentioned in section 3.6 is that for power functions (18) wih $R \leq$ 0 a fall in $P$ and the ensuing fall in $\Pi$ together lead to a net rise in $-U^{n n}(\Pi / n)^{P^{2} / n^{2}}$. This follows from

$-U^{\Pi n}(\Pi / n) P^{2} / n^{2}=-(R-I)(\Pi / n)^{R-2} P^{2} n^{-2}=(I+/ R /) \Pi^{-/ R /}(P / \Pi)^{2} n^{/ R /}$, whicl rises when $P$ falls since $P / \Pi=P /(P Q-W n-K)=I /(Q-(W n+K) / P)$ then rises. 


\section{Experimental test of the model ${ }^{45}$}

\subsection{Introduction}

The current chapter describes a series of experiments conducted to test whether the psychological predictions of the integrated model (chapter 3 ) find any support in an economic setting, and how economic and psychological mechanisms interact under certain conditions. To our knowledge this is the first attempt to directly analyze discrimination in the labor market in a laboratory experiment.

Such an experimental test faces several difficulties. First, the part of the model to be tested has to be translated into an experimental design and the important variables have to be operationalized validly. A complex theoretical model cannot be translated one-to-one into an experiment. It is always necessary to make certain simplifications when designing an experiment. The challenge is to do it such that the design captures the important aspects of the model, but does not become too complex to implement. The same holds for the operationalization of variables. The choice of a certain operationalization has to be made such that it is possible to measure or manipulate variables in a simple way, and at the same time as close as possible to the concepts of the model to be tested. In this chapter, different ways of doing this have been explored in several experiments.

First, two smaller paper-pencil studies were done to test the psychological mechanism in a competitive team setting (teams representing firms), without introducing a monetary cost for discriminatory behavior. Two teams competed against each other, and competition was varied in strength using different rules to determine the outcome for each team. The structure of the game was a social-dilemma game inside of teams, with a cooperative and an explicit non-cooperative option, i.e., a public and a private account. The two paper-pencil studies mainly differed in the operationalization of several variables (see below).

After establishing in the first two experiments that the degree of competition does in fact influence preferences, the two consecutive computerized experiments were conducted to test this effect more thoroughly and to see whether 'discriminatory'

\footnotetext{
4This chapter is based on a paper jointly authored with M. Vendrik and P. de Gijsel (2002): "The power of competition: Reducing or reinforcing discrimination?" preliminary version published in "Stability and Dynamics of Power". Conference proceedings of LAREP/SABE, 346 - 352.
} 
behavior is still found when there is a direct monetary cost connected to it. The structure of the game again was a social-dilemma game inside of teams, but without an explicit non-cooperative option. Team outcome represented the production of a product sold on a market, which was either very competitive, represented by low output prices, or not that competitive, represented by higher output prices ${ }^{46}$.

The monetary cost implemented here was established to test, whether subjects really believe so strongly in the stereotypes they have about the categories that they are willing to pay for them. Further, we wanted to see whether this is influenced by the psychological factors assumed. Willingness to pay would imply that subjects perceive the productivity differences between the categories to be so strong that the payment is compensated by the expected gains from having a more productive new team member. The suppressing economic effects of competition on this payment, as found in the theoretical model, could, as no search for individual information was possible in the experiment, only work via prejudice, but we do not assume that the artificial categories used here (see below) will evoke high amounts of prejudice.

In all four experiments subjects were categorized artificially. Artificial categories were used to avoid uncontrolled effects of existing stereotypes, such as a stereotype becoming salient which is not relevant here, but which influences behavior, e.g., the belief that economics students are greedy. Further, the use of artificial categories helps to reduce social-desirability concerns. All four experiments used a real-effort task, and the dependent variable (discrimination) was measured by asking participants whether they preferred a person of their own or the other category as a new member for their team, or were indifferent. In the computerized experiments participants were also asked whether and how much they were willing to pay for getting the preferred new member with a higher probability than 50:50, which was the default the computer 'offered'.

The design of the experiments borrows from economic and psychological experimental methodology. This leads to some difficulties, as economic and psychological principles of experimentation differ in certain respects (see, e.g., Hertwig \& Ortmann, 2001, and section $\mathbf{2 . 8 . 2}$ of this thesis). Economic experiments usually test questions about the extent to which economic actors behave 'rational' in certain (market) situations, how they decide about allocations, whether revealed preferences have certain desirable logical characteristics, etc. In all those cases it is feasible to pay subjects

\footnotetext{
${ }^{46}$ More detalied explanations of specific operationalizations are given in the resp. sections.
} 
depending on their performance, and there is generally no need for deception of the subjects. Furthermore, the concepts measured, e.g., effort, or revealed (or stated) preferences, are often relatively straightforward to operationalize. In psychological experimentation, often situations are studied were performance-based payment is not feasible, because there is no clear performance criterion. In some cases deception of the subjects about certain aspects of the experiment or the real purpose of the experiment is necessary, because otherwise measurement of the variables of interest is not or hardly possible. Finally, the operationalization of many complex psychological variables like "subjective self-relevant uncertainty" is not so straightforward.

In the two computerized experiments it was possible to pay subjects performancebased, though not for the most important decision for this study, the 'hiring' decision, but for general performance. It was, however, not possible to completely avoid deception: To avoid getting only social-desirable answers, and to link the experiments with the existing research on social-identity theory, artificial categories were used. This, however, constitutes deception about relevance of these categories, and in the first two experiments also about the way the categorization is done in general. Deception was restricted to the minimum necessary and subjects were debriefed carefully at the end of the experiment.

This chapter first describes how the experiments are linked with the theoretical model, how the hypotheses are derived, and what basic experimental design follows from this. Then, the specific designs and the results of all four studies are described in detail. The chapter concludes with a general discussion of the results of the experiments.

\subsection{General design, link with the theoretical model, and hypotheses}

\subsubsection{General design}

All experiments described here are rather exploratory in character. This means, they are not designed to test the integrated model conclusively, but rather to explore how questions derived from the general framework and the integrated model can be tested in experiments.

The four experiments reported here are supposed to look at the psychological mechanisms of identification in a competitive setting, and to analyze how competition influences the strength of the effects of these psychological mechanisms. As these experiments are just a first step in testing the model, the 'economic effect' is not yet included into the design. The general functioning of the psychological mechanisms for different strengths of competition is tested in different ways. The focus is on variables, 
which play an important role in the integrated model: Strength of competition, ingroup res. outgroup status of the new member, stereotypical perceptions about the two groups (except the first paper-pencil study), strength of identification with one's group and feelings of uncertainty. The prejudice aspect of discrimination is not measured in these experiments, as it is not very probable that prejudice plays an important role with artificial categories.

Discrimination is operationalized as any preference for a new member based on belonging to a category in a situation where no real productivity differences between categories exist. Usually, such a preference is expected to be ingroup bias, but this is not necessarily so, as the general framework shows (see figure 2.1): If only stereotypes play a role, and the ingroup is supposed to perform worse than the outgroup, outgroup bias is possible. This operationalization represents the construct of discrimination as described in the introduction (section 1.1) relatively well, as categories do not provide relevant information in the experiment, and therefore, having a preference for the ingroup (or the outgroup) is discrimination in the sense that 'equals' with respect to the existing knowledge about productivity are treated unequally ${ }^{47}$. The fact that participants do not have any individual information about the possible new members and also cannot acquire it is a deviation from the situation the theoretical model describes in chapter 3 and is along with the fact that prejudice is not assumed to play a role - the second reason why no economic effect of competition in the sense of the theoretical model can work here. However, this was the easiest way to guarantee that subjects have the same average information about members of both groups ${ }^{48}$. Giving information about individual members of the two groups could lead to evaluative differences between the categories, which of course would distort the mechanisms assumed ${ }^{49}$. At no point an explicit and clear link between performance and category membership is drawn, such that subjects could not even know what kind of productivity-related information might be inferred from the categories.

\footnotetext{
${ }^{47}$ Of course there might be individual differences between the subjects with respect to productivity, but these differences cannot systematically be related to category membership, and they are not known.

${ }^{4}$ This is necessary to be able to test for discriminatory preferences by testing whether some participants prefer one to the other category as a new member. If all subjects have the same information on both categories, differences in preferences for one or the other category can only result from differences in discriminatory preferences, and not from differences in information about members of the two groups.

49 This could happen in an experiment with artificial categories and no a priori evaluative differences between the categories, because subjects would start to develop a picture of the categories in the experiment based on the individual information they get. This is not relevant for real-life situations, at least in the short run, as described in section 2.3.1.
} 
Competition as the main independent variable was operationalized differently in the paper-pencil and the computerized experiments. In the paper-pencil studies, teams actively competed against each other. This comes close to the 'process' definition of competition as described in chapter 2. To vary the strength of competition between treatments, in one treatment only the 'winning' team got any points ("winner takes all", WTA). This represents strong competition, as the 'looser' does not get anything and therefore it is very important for each team to become the 'winner', In the other treatment, both teams got points corresponding to their relative performance ("proportional representation", PR). In that case it is less important to win or to do better than the other team, and therefore, this treatment is assumed to represent weaker competition. Pretests were done where subjects got descriptions of both treatments and were asked to rate in which treatment competition is stronger. Participants in fact did perceive differences in strength of competition as intended.

In the computerized experiments, competition was operationalized more in the sense of the definition used in the integrated model, as either high or low output prices. High output prices can be a result of weaker competition, while low output prices can result from strong competition (on the supply side). Teams still had to play the game as good as possible to get as many points (money) as possible, so that competition still was an activity, even if there was no specific other team against which they were competing. Still, in a situation of high output-prices it is not so necessary for the team to do well and to 'produce' as much as possible, because already for low output a relatively high amount of money is earned. On the other hand, when output prices are low, a lot of output is necessary to make some decent earnings. In fact, the two operationalizations of competition were not that different, because the situation of subjects in WTA and PR implied expectations of lower or higher earnings (in experimental points), just like lower or higher output prices did (translated into lower or higher chances of getting a cinema voucher in the first experiments). As in WTA the variance of points which could be won also was much higher than in PR, in the paper-pencil studies stronger competition also implied higher risk, which probably has an even stronger effect on self-relevant uncertainty than low output prices do.

Uncertainty in all experiments existed in the sense of 'risk of getting no (or low) rewards' in experiments 3 and 4 resp. making a lower chance to win the cinema voucher in experiments 1 and 2 . The operationalization of the other variables measured is 
described for each experiment separately below, because the variables used and their operationalizations differ slightly between the experiments.

While the paper-pencil studies were designed to investigate the general existence of a psychological effect of competition on discriminatory preferences in the absence of a monetary cost, the computerized experiments more closely tried to test some of the predictions of the (psychological part of the) theoretical model from chapter 3, especially testing also for discriminatory behavior ${ }^{50}$. They did so by allowing for the psychological mechanisms to work while modeling different degrees of competition by different levels of output prices. In addition, it was made costly to implement discriminatory preferences, such that it could be seen whether the stereotypical perceptions developed were seen as so trustworthy that getting the 'better' new member would compensate for the cost subjects incurred. Note again that this does not imply the 'economic effect' of competition with respect to stereotyping, according to the theoretical model, as this effect can only work when it is possible to search for individual information about candidates (see chapter 3 and below in this chapter).

\subsubsection{Link with the theoretical model}

In order to avoid effects of socially desirable answers, each team's discrimination coefficient $D^{*}$ was not measured directly in the computerized experiments. The discrimination coefficient $D^{*}$ is now given by output price $P$ times the stereotype-based part $S^{*} \triangle M P$ of the perceptions $\triangle M P_{i}^{*}$ of productivity differences between particular pairs of candidates from the ingroup and the outgroup. The use of artificial categories made an emergence of strong emotional prejudices less probable, which allowed us to neglect the second component in expression (3.20) in chapter 3 for the discrimination coefficient $D^{*}$.

Since there is no individualized information on actual productivity differences in the experiments, non-zero perceptions $\triangle M P_{i} *$ can only be based on a stereotypic perception $\triangle M P$ of differences in average productivity between ingroup and outgroup. However, since the categories are artificial and do not provide relevant information on productivity differences in the experiments, subjects may not fully rely on their stereotypic perceptions and hence give a weight smaller than one to these perceptions in the formation of their $\triangle M P_{i}^{*}$. Such a weight can be considered as a proxy of $S^{*}$ in the

\footnotetext{
${ }^{50}$ This is done because experimental psychologists often doubt whether there is a direct link between stated 'attitudes' and behavior (see, e.g., Eagly \& Chaiken, 1998).
} 
theoretical model. Thus, in the experiments perceptions $\Delta M P_{i}{ }^{*}$ are assumed to be given by $S^{*} \Delta M P$, i.e. by eq. (3.4) in Chapter 3 with $\Delta M P^{T},=0$.

When the intensity of competition varies both $S^{*}$ and $\triangle M P$ may change, but in the experiments we could only determine the direction of the total change in $\Delta M P_{1} *=$ $S^{*} \Delta M P$. This direction was inferred from differences in the amount of money that subjects said they were willing to pay as a team for getting a preferred new member with a higher probability than the initial 50:50. Since we wanted to measure the direction of change in $S^{*} \Delta M P=D^{*} / P$ rather than that in $D^{*}$ itself, the amounts of money $\tilde{D}$ that subjects were willing to pay for getting a preferred new member with a higher chance had to be expressed as fractions or percentages of the output price $P$.

The relation between the experimental $\tilde{D} / P$ and the theoretical $D * P$ can be derived as follows. Assume that the subjects choose $\widetilde{D} / P$ approximately in such a way that it maximizes the expected profit gain of their team from getting a preferred new member or not, $E[\Delta R-\tilde{D}]=E \Delta R-\widetilde{D} \cdot{ }^{51}$ Here $\Delta R$ is the perceived additional revenue from getting a preferred new member or not over the three rounds that are played with the new member, i.e. $\triangle R=3 P \Delta M P_{i}=3 P S \cdot \Delta M P$ when a team member from the preferred category is assigned to the team, and $\Delta R=0$ when a team member from the other category is assigned. Thus, $\Delta R$ is stochastic. The probability $\rho$ of getting a new member from the preferred category depends on $\widetilde{D} / P$ as

$$
\begin{array}{ll}
\rho=0.5+\beta \tilde{D} / P & \text { for } \widetilde{D} / P \leq \widetilde{D}_{\max } / P=1 /(2 \beta), \\
\rho=1 & \text { for } \widetilde{D} / P \geq \widetilde{D}_{\max } / P=1 /(2 \beta),
\end{array}
$$

where $\beta$ is a positive parameter.

Hence,

$$
\begin{aligned}
E \Delta R-\widetilde{D} & =\rho 3 P S \bullet \Delta M P+(1-\rho) 0-\widetilde{D}=3(0.5+\beta \tilde{D} / P) P S \bullet \Delta M P-\widetilde{D}= \\
& =(3 \beta S \cdot \Delta M P-1) \widetilde{D}+1.5 P S \bullet \Delta M P
\end{aligned}
$$

\footnotetext{
"St Strictly speaking, subjects should maximize $E[(1 / 5) \Delta R-(1 / 4) \tilde{D}]$ since revenues are shared among the five members of the extended team including the new member, whereas the payment $\tilde{D}$ is shared among the four old members. However, we assume that subjects do not take this minor complication into account in their choice of $\tilde{D}$.
} 
for $\tilde{D} / P \leq 1 /(2 \beta)$, and $E \Delta R-\tilde{D}=3 P S * \Delta M P-\tilde{D}$ for $\tilde{D} / P \geq 1 /(2 \beta)$. The latter function is decreasing in $\widetilde{D}$, while function (4.2) is linearly increasing or decreasing in $\widetilde{D}$, dependent on the sign of the expression in front of $\widetilde{D}$. Thus, we have three cases:

(i) $3 \beta S^{*} \triangle M P<1$ or $S^{*} \Delta M P<I /(3 \beta)$

(ii) $3 \beta S^{*} \triangle M P=1$ or $S^{*} \triangle M P=1 /(3 \beta)$

(iii) $3 \beta S^{*} \triangle M P>1$ or $S^{*} \triangle M P>1 /(3 \beta)$

In case (i) function (4.2) is linearly decreasing in $\widetilde{D}$, implying that $E \Delta R-\widetilde{D}$ is maximal for $\widetilde{D} / P=0$, so perception $\triangle M P_{i}^{*}=S^{*} \Delta M P$ is not large enough to make it advantageous to pay an amount of money to raise the chance of getting a new team member from the preferred category. In case (iii) function (4.2) is linearly increasing in $\widetilde{D}$, implying that $E \Delta R-\widetilde{D}$ is maximal for $\widetilde{D} / P=\widetilde{D}_{\max } / P=1 /(2 \beta)$, so perception $\Delta M P_{i} *$ $=S^{*} \triangle M P$ is large enough to make it advantageous to pay the amount of money necessary to raise the chance of getting a new team member from the preferred category to one. In the intermediate case (ii) function (4.2) is constant with respect to $\widetilde{D}$, implying that $E \Delta R-\widetilde{D}$ is maximal for all values of $\widetilde{D} / P$ between 0 and $I /(2 \beta)$. Plotting the optimal $\tilde{D} / P$ as a function of $S \bullet \Delta M P=D / P$, we then get the stepwise graph in figure 4.1. 


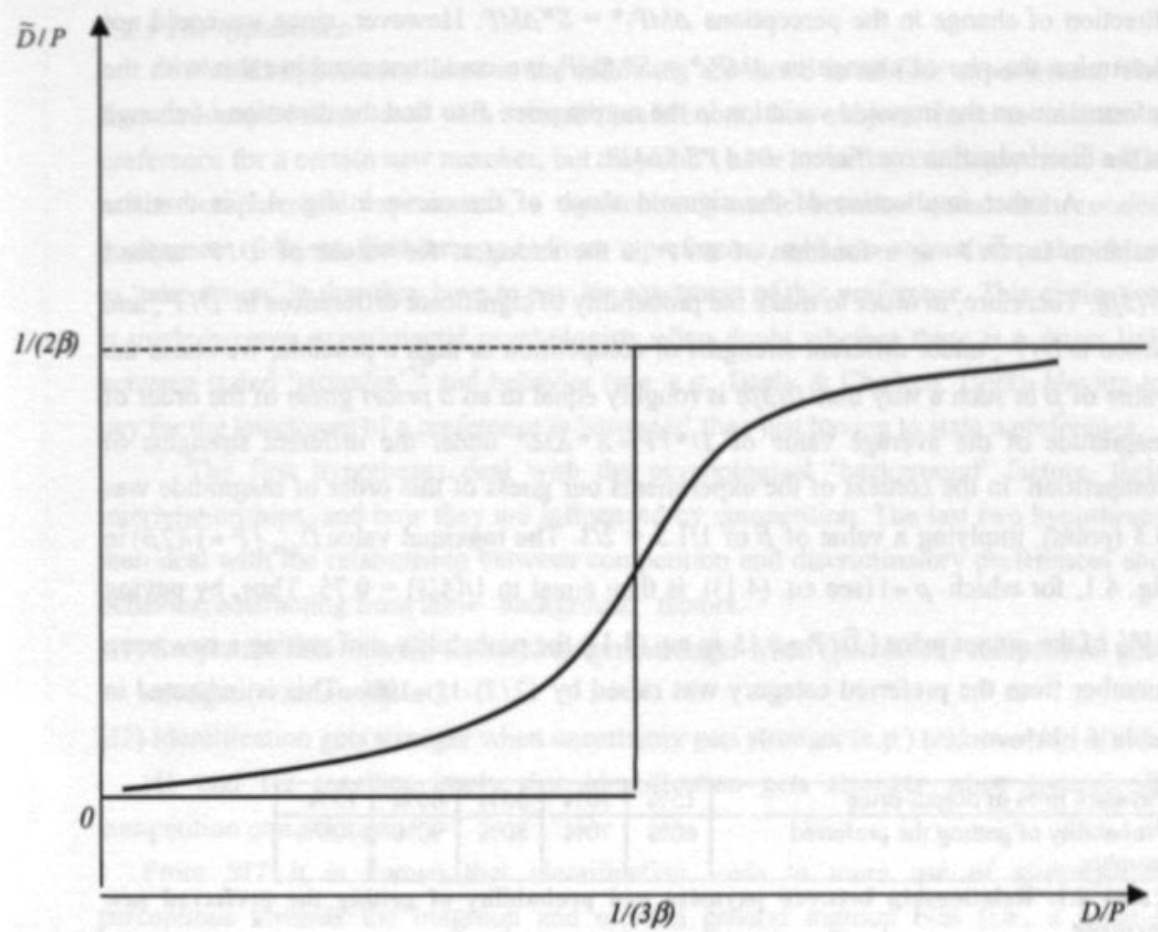

Figure 4.1: The optimal $\tilde{D} / P$ as a function of $S * \Delta M P=D / P$

However, we can safely state that the subjects in the experiment chose their $\widetilde{D} / P$ not on the basis of such a precise calculation, but in a much more intuitive way. On the other hand, we may assume that their intuition led to choices of $\tilde{D} / P$ which form a smooth approximation of the stepwise graph in fig. 4.1. Such an approximation is indicated by the bold smooth sigmoid curve in fig. 4.1. A curve of this shape implies that the experimental $\widetilde{D} / P$ is a monotonously increasing function of the theoretical $D / P$ for not too low and not too high values of $D / P$. Conversely, this means that a higher value of $\tilde{D} / P$ found in the experiments implies a higher value of the underlying $D \cdot / P=S * \triangle M P$. Thus, under the assumptions made above, a significant direction of change in $\widetilde{D} / P$ found in the experiments gives an unambiguous indication of the 
direction of change in the perceptions $\triangle M P_{t}^{*}=S^{*} \Delta M P$. However, since we could not determine the size of change in $\triangle M P_{i}^{*}=S^{*} \Delta M P$, we could not combine this with the information on the imposed variation in the output price $P$ to find the direction of change in the discrimination coefficient $D^{*}=P S * \Delta M P$.

Another implication of the sigmoid shape of the curve in fig. 4.1 is that the variation in $\tilde{D} / P$ as a function of $D / P$ is the strongest for values of $D / P$ around $1 /(3 \beta)$. Therefore, in order to make the probability of significant differences in $\tilde{D} / P$, and hence in $D / P$, under different strengths of competition as high a possible, we chose the value of $\beta$ in such a way that $l /(3 \beta)$ is roughly equal to an a priori guess of the order of magnitude of the average value of $D^{*} / P=S * \Delta M P$ under the different strengths of competition. In the context of the experiments our guess of this order of magnitude was 0.5 (point), implying a value of $\beta$ of $1 / 1.5=2 / 3$. The maximal value $\widetilde{D}_{\max } / P=1 /(2 \beta)$ in fig. 4.1 , for which $\rho=1$ (see eq. (4.1)), is then equal to $1 /(4 / 3)=0.75$. Thus, by paying $15 \%$ of the output price $(\widetilde{D} / P=0.15$ in eq. (4.1)) the probability $\rho$ of getting a new team member from the preferred category was raised by $(2 / 3) \cdot 15=10 \%$. This is indicated in table 4.1 below.

\begin{tabular}{|l|l|l|l|l|l|}
\hline Payment in \% of output-price & $15 \%$ & $30 \%$ & $45 \%$ & $60 \%$ & $75 \%$ \\
\hline $\begin{array}{l}\text { Probability of getting the preferred } \\
\text { member }\end{array}$ & $60 \%$ & $70 \%$ & $80 \%$ & $90 \%$ & $100 \%$ \\
\hline
\end{tabular}

Table 4.1: Relationship between payment and probability of getting the preferred new member

The strength of preference subjects indicate in the experiments before indicating their willingness to pay represents the disutility $D U^{*}$ of hiring an outgroup member, as given by eq. 3.22 of the theoretical model. The higher the preference subjects indicate, the higher they perceive their disutility $D U^{*}$ of hiring a new member of the non-preferred category instead of a new member of the preferred category. $D U^{*}$ depends on two factors which are assumed to be influenced by competition: (i) The expected productivity difference $S^{*} \Delta M P$ and (ii) the subjective importance of $S^{*} \Delta M P$ in their preferences,

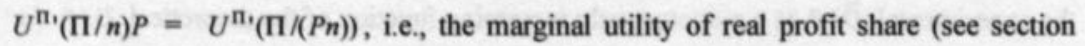
3.4.1). Competition is assumed to influence both in the same direction - the use $\mathrm{S}^{*}$ of the stereotypic percetion $\triangle M P$ goes up due to the psychological mechanisms assumed, while an 'economic' mechanism leads to higher marginal utility of the real profit share (or the share in points), i.e., to a higher $U^{\mathrm{n}_{1}}(\mathrm{\Pi} /(P n))$. 


\subsubsection{The hypotheses}

Not all hypotheses listed in the following are tested in all four experiments. The first two experiments deal with 'stated' preferences, i.e., subjects have to indicate a preference for a certain new member, but they don't have to 'act' accordingly later, while in the computerized experiments, a distinction is made between stated and revealed preferences. Subjects first have to indicate a preference, and in a second step, they have to 'take action' in that they have to pay for enactment of this preference. This distinction is made because experimental psychologists often doubt whether there is a direct link between stated 'attitudes' ${ }^{, 52}$ and behavior (see, e.g., Eagly \& Chaiken, 1998). Having to pay for the enactment of a preference is 'stronger' than just having to state a preference.

The first hypotheses deal with the psychological 'background' factors, their interrelationships, and how they are influenced by competition. The last two hypotheses then deal with the relationship between competition and discriminatory preferences and behavior, abstracting from these 'background' factors.

H1) Subjective self-relevant uncertainty gets stronger when (perceived) competition gets stronger (relationship a)).

H2) Identification gets stronger when uncertainty gets stronger (c.p.) (relationship b)).

$\mathrm{H} 1$ and $\mathrm{H} 2$ together imply that identification gets stronger when (perceived) competition gets stronger.

From SIT it is known that identification leads to more use of stereotypical perceptions towards the outgroup and often a general ingroup bias (i.e., a general preference for the ingroup as opposed to the outgroup, including more positive perceptions, emotions, and sometimes preferential behavior). Because of artificial categories we do not expect a (strong) influence of prejudice here. Therefore, it follows:

H3) Under strong as compared to weak competition, a new member from the ingroup is preferred (more strongly) over a new member from the outgroup. (relationships c) and e) and antecedents).

H4) Under strong as compared to weak competition, more payments are made for members of the ingroup (relationships $c$ ), d), e) and antecedents).

Hypotheses 3 and 4 are assumed to hold because due to identification and stereotyping the ingroup is perceived as performing better on average than the outgroup.

\footnotetext{
${ }^{52}$ Now used in the more general sense, not just as an emotional evaluation.
} 
Psychological mechanism:

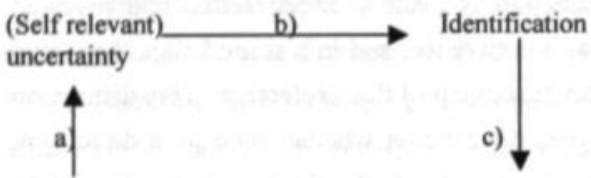

Competition

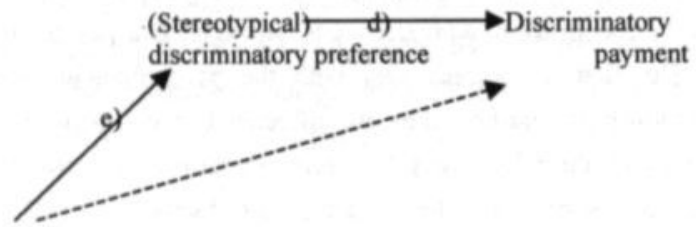

Marginal utility of points earned

'Economic' mechanism:

Figure 4.2: Assumed relationships between variables - straight lines represent positive relationships, dashed lines negative relationships.

\subsection{The paper-pencil studies}

As the first two experiments were rather 'preliminary studies' for the computerized experiments, aiming just at testing whether the psychological relationships assumed apply at all, they are reported rather shortly together.

\subsubsection{Subjects}

In experiment 1,53 undergraduate students (20 male and 31 female, two of unknown sex) at the Faculty of Economics and Business Administration of the University of Maastricht participated in groups of 8 respectively 10 people. Participants in experiment 2 were 60 ( 24 males, 36 females) undergraduate students from various faculties at the University of Heidelberg, Germany. 


\subsubsection{Material}

The first experiments were paper-pencil studies. In experiment $l$, the task to distribute subjects into categories was taken from the minimal-group paradigm (Tajfel et al, 1971). Subjects had to estimate the number of points on slides presented to them shortly, and then were categorized in over- and underestimators ${ }^{53}$. The real effort task teams had to do consisted of a concentration test from clinical psychology, the "d2-test" (Brickenkamp, 1962), which does not require specific skills apart from concentration.

In experiment 2, the categorization task was a questionnaire on "cognitive style" (constructed similar to the "Paragon educational consulting student learning style inventory"). The task in the game was now skill-dependent - logical problems taken from an intelligence test, the "CFT3" (Cattell \& Weiss, 1971). The fact that now both the categorization task and the real-effort task were cognitive in character, made it possible to link belonging to a category with performance on the task even if there did not exist any real link, as categorization was in fact done randomly (see footnote 53).

In both experiments subjects got two pages of the task in each round, one for a 'private' account and one for a 'team' account. They could decide themselves whether to contribute rather to the team account or to their private account. The structure of the game thus was inter-team competition combined with an intra-team social-dilemma game. This structure was mainly chosen because in real-effort tasks subjects always can choose not to exhibit much effort for the team, whether there is an 'explicit' noncooperative option or not (they can just sleep, for example). We decided to use an explicit non-cooperative option here to detect free riding.

The 'discrimination' measure consisted of one question asking subjects whether they would prefer a new member to be rather from their own or the other category (i.e., over- vs. underestimator in experiment 1 , cognitive style $\mathrm{K}$ vs. $\mathrm{P}$ in experiment 2 ). This question had to be answered by making a cross on a line between the two extreme points, such that making a cross in the middle indicated indifference (see appendices I - III for the complete instructions).

Uncertainty, perceived strength of competition, and identification were measured in experiment 1 and 2 using either self-constructed items or items from existing scales: In experiment one, perceived competition and uncertainty were measured at two points in time, using self-constructed questions ("How strong is the competition between the teams

\footnotetext{
53 This categorization was deceptive, as all subjects playing in the main teams were told to be overestimators. Categorization was the only instance of deception in the first experiments.
} 
in your perception?", "How do you feel about your final result (points) in this game?"). Questions about identifcation with the category were adapted from Mullin \& Hogg (1998) ("What do you think, how much you might like other overestimators?" "How similar do you think you might be to other overestimators in terms of general attitudes and opinions?" "How much would you like to get to know other overestimators?" "How much do you feel to belong to other overestimators?"). Three items adapted from Mullin \& Hogg (1998) assessed social awareness, a proxy for social-desirability concerns among participants. ("How much aware are you of the presence of others?" "How much do you feel observed by others?" "How much do you feel observed by the experimenter?").

For experiment 2, questions were translated into German. Uncertainty and competition were measured only at one point in time, using some more questions than in experiment 1, to try to capture the complexity of both constructs (competition: "How high is in your opinion the chance in this game that one team has to leave after half-time evaluation? 34 ", "How high is in your opinion the chance in this game that both teams can be content with their amount of points (team points!) after half-time evaluation?", "How high is in your opinion in this game the chance that you personally are content with your points (team and private points) at half-time evaluation?" "How important for wining in this game is it that all team members do their best?". "How strong is in your opinion in this game competition between the teams?"; uncertainty: "How certain are you that you can reach your aims in this game?" "How certain are you that your effort was sufficient to let the team continue after half-time evaluation?", "How certain are you that the other team members did exhibit enough effort so that the team can continue after half-time evaluation?", "How certain are you that the team can continue after half-time evaluation?"). Importance to win the game was assessed using one self-constructed question ("How important is it for you that your team can continue after half-time evaluation?"). This variable was measured, as it seemed plausible that subjects who do not think that it is important to win in the game perceive the incentive structure differently and thus behave differently. Therefore, it is important to be able to identify them.

In both paper-pencil studies, the chances of earning money were not equal for all teams, because the 'spare' teams, which were necessary for the 'discrimination measure' (see below), did not participate in the competition. Payment therefore was not done

\footnotetext{
${ }^{54}$ Half-time evaluation is the point at which during the experiment it was evaluated whether each team had done well enough to continue playing the game (see below for a more detailed explanation).
} 
performance-based. Participants got 10 Dutch guilders/10 DM for participation, and they knew this from the beginning. To enhance motivation to exhibit effort in the game, the best five participants, concerning both team and private points, could win a cinema voucher.

\subsubsection{Procedure}

In both paper-pencil studies, competition between teams had either a strongly competitive 'winner takes it all' (WTA) or a less competitive 'proportional representation' (PR) structure. In WTA, only the winning team gets any points. In PR, teams get paid according to their relative amount of points. This leads to different perceived strengths of competition and also different strengths of uncertainty. In WTA, the team is uncertain whether it can manage to get any points at all, while in PR, teams are 'only' uncertain about the amount of points they will get. Expected total points should be higher in PR than in WTA as well, as has been explained before.

In experiment 1 , subjects first read the instructions, and then did the categorizing task. While an assistant grouped participants into teams, they played a test round of the game in randomly assigned teams. After the winner of the test round was determined, subjects filled in a questionnaire about their perception of the situation, containing the first competition and uncertainty measurement. Then, subjects got information about team composition. They learned that all members of their team were of the same category. On average three people formed a team, and two teams competed in each session. Two more people were playing in a third team, which was needed for the discrimination measure. This 'spare' team did not participate in the competition, but played the game as well. It was necessary because in the discrimination measure subjects made a decision which kind of new member they wanted for their team. To be able to make participants believe that this decision would be implemented later, potential 'new members' were necessary. They should have some experience with the game as well, because otherwise they could be expected to perform worse than 'old' members. To achieve this, the 'spare' team was used. A further advantage of the 'spare team' was that it was less obvious for subjects with whom they were playing, as more participants were in the room.

After the first 'counting' round of the game was played, participants filled in the 'discrimination measure'. They further answered questions measuring identification with the team and the category they belonged to, social awareness and perception of the 
situation, especially with respect to uncertainty and competition (see above and appendix I for these questions). After this, the experiment was ended, participants were debriefed and paid, and they got their 'lottery ticket' for the cinema voucher.

The procedure of experiment 2 was essentially the same. The main difference was the way of categorizing participants, as described above. Subjects also played more rounds - apart from the test round, three rounds were played before the discrimination measure was administered. Competition was strengthened for the WTA treatment, to achieve a more pronounced effect: A team had to reach a minimum of 30 points per person on average in the team task at the point of a half-time evaluation (after two rounds, but that was not known to participants), otherwise, the third, spare team would replace it. In the PR treatment this requirement was fulfilled easily, but it was hard to reach in WTA. As participants could not be sure that both teams were equally strong and moreover were not precisely informed, after how many rounds half-time evaluation would take place, there was uncertainty about probabilities, i.e., ambiguity.

\subsubsection{Results}

The paper-pencil studies were analyzed aggregated by teams, as within teams individual data are not independent because subjects got feedback about their performance as a team. Aggregating the data resulted in a relatively small number of observations per treatment, which is normal for exploratory experiments. However, the number of observations became even smaller, as for several reasons it was necessary to exclude some teams from the analysis. In the first experiment five teams had to be excluded because of language problems ${ }^{55}$ - teams with more than one fourth of participants not having English, German, or Dutch as a mother language were excluded $^{56}$. In the second experiment we controlled additionally for 'importance of winning the game', and teams scoring in the lowest third were excluded. This resulted in the first experiment in a sample of ten teams for analysis, six in WTA and four in PR, and in the second experiment in a sample of 13 teams, six in WTA and seven in PR. Because of the small number of observations, treatments were compared using non-parametric Man-Whitney-U tests. For significance testing, the standard levels of significance were used, including the 'marginally significant' $10 \%$ level. Marginally significant results

\footnotetext{
55 The Faculty of Economics and Business Administration of the University of Maastricht is an international faculty offering courses in English. Students come from all over the world with different mastery of English.

${ }^{6}$ Students with these mother languages usually have a thorough knowledge of English.
} 
have to be interpreted carefully because the risk of falsely rejecting the null-hypothesis (alpha-error) is high. We decided still to report results significant on this level, as, on the other hand, with small samples the danger exists - especially when using non-parametric tests - that results do just not get significant, even if there is an effect (beta-error).

\section{Experiment I}

Descriptive statistics for both treatments are given in the following table.

\begin{tabular}{|c|c|c|c|c|c|c|}
\hline \multirow{2}{*}{$\frac{\text { Treatment }}{\text { WTA }}$} & \multirow{2}{*}{\multicolumn{2}{|c|}{$\begin{array}{c}\text { Preference for new } 6 \\
\text { member }\end{array}$}} & \multicolumn{3}{|c|}{ Minimum Maximum Mean } & \multirow{2}{*}{$\frac{\text { Std. Dev. }}{1.82}$} \\
\hline & & & 2.57 & 6.65 & 4.62 & \\
\hline \multirow{9}{*}{ PR } & Competition & 6 & 4.28 & 7.83 & 5.61 & 1.42 \\
\hline & Uncertainty tI & 6 & 3.30 & 8.17 & 5.28 & 1.77 \\
\hline & Uncertainty 12 & 6 & 3.25 & 5.37 & 4.64 & .76 \\
\hline & Identification & 6 & 4.75 & 6.00 & 5.31 & .47 \\
\hline & $\begin{array}{l}\text { Preference for new } \\
\text { member }\end{array}$ & 4 & 1.58 & 3.43 & 2.48 & 88 \\
\hline & Competition & 4 & 3.05 & 6.33 & 4.79 & 1.53 \\
\hline & Uncertainty $\mathrm{tl}$. & 4 & 2.43 & 4.30 & 3.27 & .89 \\
\hline & Uncertainty $\mathrm{t} 2$. & 4 & 3.55 & 4.40 & 3.98 & 43 \\
\hline & Identification & 4 & 5.00 & 6.44 & 5.44 & 67 \\
\hline
\end{tabular}

Table 4.2: Descriptive statistics

Hypothesis I) (relationship a) in figure 4.2): No significant difference in perceived uncertainty between the two treatments could be found, neither at time 1, nor at time 2. Uncertainty (at time one and at time 2) does also not correlate with perceived competition at both times.

Hypothesis 2) (relationship b) in figure 4.2): No significant difference in identification with the own category between the treatments was found. Identification with the own category correlates marginally significantly with perceived uncertainty measured at time $1(\mathrm{r}=.60, \mathrm{p}=.067(2 \text {-tailed }))^{57}$, but not with perceived uncertainty measured at time 2 and not with the combined measure of uncertainty (the average of both times). This means, the less certain someone is at time 1 , the more he identifies with his category (to reduce the feeling of uncertainty $\Psi$ ). Thus, at time 2 , the remaining high uncertainty is not related to low identification. This confirms hypothesis 2 .

To test the corollary from hypothesis 1 and 2 , identification was correlated with perceived competition. Identification does correlate significantly with perceived competition (aggregated over both times of measurement), such that the stronger

\footnotetext{
${ }^{57}$ Correlations were always calculated using Pearson correlation-coefficients $(r)$.
} 
competition is perceived, the stronger subjects identify with their category $(r=.62, p=$ .026 (1-tailed)).

Hypotheses 3) (relationships c) and e) in figure 4.2): The difference in preference for a new member between the treatments is not significant $(\mathrm{Z}=-1.23$, as. sign. $=.221$ $\left(2\right.$-tailed) ${ }^{58}$ ). But, in PR (when competition is weak) preference for a new member differs significantly from indifference (5), which is not the case in WTA. The direction of this preference is surprising: It tends towards outgroup bias $(P R: t=-3.97, p=.029$, WTA: $t=$ $-1.04, t=.359(2$-tailed) $)$.

Exploratory analyses: A surprise was that in experiment 1 hardly any free-riding was observed.

To check whether the manipulation of 'strength of competition' worked, perceived strength of competition was compared for the two treatments. No significant difference was found, though it tends in the direction expected, being higher in WTA ( $\mathrm{Z}$ $=-.98$, as sig. $=.164$ (1-tailed)). The manipulation check thus unfortunately does not confirm that the manipulation of competition worked as intended. This does, however, not constitute a bigger problem, as it is still possible to correlate the relevant variables with perceived competition to test the predictions made.

\section{Experiment 2:}

The following table shows descriptive statistics for experiment 2.

\begin{tabular}{|c|c|c|c|c|c|c|}
\hline Treatment & & $\mathrm{N}$ & Minimum & Aaximu & Mean & Std. Dev \\
\hline \multirow[t]{4}{*}{ WTA } & $\begin{array}{r}\text { Preference for new } \\
\text { member }\end{array}$ & 6 & 5.10 & 8.10 & 6.53 & 1.05 \\
\hline & Uncertainty & 6 & 4.16 & 6.03 & 5.14 & .65 \\
\hline & Identification & 6 & 4.33 & 5.17 & 4.82 & .32 \\
\hline & Competition & 6 & 5.41 & 6.23 & 5.74 & .30 \\
\hline \multirow[t]{4}{*}{ PR } & $\begin{array}{r}\text { Preference for new } \\
\text { member }\end{array}$ & 7 & 1.10 & 7.40 & 4.19 & 2.37 \\
\hline & Uncertainty & 7 & 3.25 & 7.33 & 5.20 & 1.29 \\
\hline & Identification & 7 & 3.33 & 5.08 & 4.44 & .64 \\
\hline & Competition & 7 & 3.91 & 6.74 & 5.14 & 1.01 \\
\hline
\end{tabular}

Table 4.3 Descriptive statistics

Hypothesis 1) (relationship a) in figure 4.2): No significant difference in subjective uncertainty exists between the two treatments, but perceived competition is

\footnotetext{
\$s This has been tested two-tailed, as already the means showed that the effect would not be in the direction expected.
} 
correlated with uncertainty: The higher perceived competition, the higher is uncertainty, which confirms hypothesis $1(\mathrm{r}=.79, \mathrm{p}=.001$ (1-tailed)).

Hypothesis 2) (relationship b) in figure 4.2): Identification with the group does not significantly differ between the treatments. It also does not correlate significantly with uncertainty. Hypothesis 2 thus could not be confirmed.

This also implies that the combination of hypotheses 1 and 2 does not hold as expected, i.e., identification does not correlate significantly with perceived competition.

Hypotheses 3) (relationships c) and e) in figure 4.2): The difference in preference for a new member between the two treatments is marginally significant $(Z=-1.86, p=$ .063 (2-tailed)). Contrary to experiment 1, in this experiment it seemed to make some sense to link performance in the task and category membership. The result is then also partially the contrary of the result in experiment 1: Only in WTA preference for a new member differs significantly from indifference $(t=3.59, p=.016(2$-tailed)), again towards outgroup bias. In PR subjects are indifferent between the two categories $(t=$ $91, \mathrm{p}=.400$ (2-tailed)).

As the difference in perceived strength of competition between the two treatments is not very strong, we also tested whether perceived competition correlates with the discrimination measure. Perceived competition is significantly correlated with preference for a new member, in a direction which is expectable from the preceding analysis: The stronger competition is perceived to be, the more an outgroup member is preferred $(r=$ $.60, \mathrm{p}=.031$ (2-tailed)).

Exploratory analyses: Similar to experiment 1, in experiment 2 hardly any freeriding was observed.

The difference in perceived competition between treatments becomes marginally significant in the expected direction $(\mathrm{Z}=-1.42$, as. sig. $=.077$ (1-tailed)), i.e., the manipulation check this time confirms that manipulation of competition between the treatments worked.

\subsubsection{Discussion}

Despite of the small sample, experiment I showed a slight difference in subjects' preferences between the two treatments. In WTA, subjects on average were indifferent between the categories, whereas in PR subjects positively discriminated for new members from another category. This is basically the opposite from what was expected. 
The most probable reason for this effect is the use of artificial categories, which could not be linked to performance in the task. Therefore, belief in the informational content of the categories was very low. In PR, subjects might have been curious whether outgroup members might differ from them, they showed risk-seeking behavior, because there was not much at stake. In WTA, where it was more important to have a good team, subjects did not want to take the risk to make a mistake when choosing - in the sense of Camerer \& Weber (1992), they did not want to take the risk of having the wrong belief about the probability distribution of productivity differences - and risk-seeking behavior disappeared. That discrimination against the outgroup was not observed at all is probably due to the artificial categories, because overall identification is low with such categories.

Differences in the psychological factors between the two treatments were not very strong. But, it became visible that perceived strength of competition does play a role in the way expected: Identification with the category correlates with perceived competition and uncertainty at time 1. Strong perceived competition, i.e., strong perceived uncertainty, in the beginning thus probably makes subjects identify with their group, which reduces perceived subjective uncertainty measured at later times.

Experiment 2 showed that in situations where choosing someone form the opposite category seemed to have some advantage, subjects tended to do so - but only under strong competition. The fact that categories were generated such that one could assume that they have some informatory content with respect to productivity turned the pattern to the contrary of experiment 1's - strong preferences in WTA, no preferences in PR. This can be interpreted as a 'weak' kind of statistical discrimination: Subjects wanted members of the group they thought would perform better in the task, but this performance estimation was based on stereotypical perceptions, not on real, known, differences between the groups. With a rather complicated cognitive task it obviously made sense for the participants to think that maybe someone of the opposite cognitive style would do the task even better. When competition, and thus importance to make a good decision, was low, subjects were indifferent.

The correlation of perceived competition with preference and with uncertainty partly confirms the hypotheses about the psychological mechanisms behind discriminatory behavior, but discrimination in WTA also followed a 'rational' motive of performance, when subjects believed in the informational content of the categories. This shows rather 'economic' thinking of the participants; it constitutes a weak kind of 
statistical discrimination, which - different from assumptions of standard statistical discrimination theories - only occurred when competition was strong.

That no correlation of perceived uncertainty with identification was found might result from a floor effect: Identification with the categories was very low (mean $=4.6$, std.dev. $=.54)^{59}$. Obviously, subjects did not perceive identification with the artificial categories as useful to reduce subjective uncertainty.

The paper-pencil studies thus indicate that strengthening of competition has an effect on preferences. Taken together they also indicate that this effect might be linked to uncertainty evoked by competition. The direction of the effect competition has on discriminatory preferences is determined by (belief in) the informational content of the categories with respect to performance in the task. Experiment 2 showed that 'weak' statistical discrimination (i.e., statistical discrimination based on wrong ${ }^{60}$ beliefs) can be $^{2}$ reinforced by the strengthening of competition, when subjects believe in the informational content of the categories. Standard economic theories of statistical discrimination do not mention that this form of discrimination can become stronger when competition strengthens, and surely not, when it is based on wrong beliefs. The reason for this finding here probably is that, given the psychological mechanism of strengthened use of (and belief in) the categories as a source of information, the economic mechanism of statistical discrimination works with more force. As in an experiment the importance of decisions in general is relatively low, and therefore, the importance of productivity considerations is low as well (as the theoretical model predicts), no statistical discrimination occured in PR.

\subsection{The computerized experiments}

For the computerized experiments the design was slightly changed. After it had been established in the first two experiments that competition does have an effect on preferences, and that subjects use artificial categories as a basis for decision-making. especially when they can be linked with performance, the idea was now to test more in detail some of the psychological predictions of the integrated model from chapter 3 . To do this, an experimental design more closely related to the integrated model was used (see also section 4.2.2). This also implied a different operationalization of competition,

\footnotetext{
"Identification was measured on a 9-point scale ranging from 'not very much' to 'very much'.

${ }^{6}$ Beliefs were wrong in that subjects had no information about the two groups apart from the category information, and category information is not performance-related.
} 
using now high and low output prices as a proxy for weak and strong competition (see below).

Subjects again played a real-effort game in teams. The explicit non-cooperative option (the private task in the first two experiments) was abandoned, to make the experimental situation less complex overall. After hardly any free riding happened in the first two experiments, it did not seem necessary anymore to have such an explicit noncooperative option.

The computerized experiments were designed such that it would be possible to control the effect the stereotype of the categories had, i.e., participants were provided with a stereotype of the two categories blue and red, the (evaluative) content of which could be known. In experiments 1 and 2, it was impossible to know for the experimenter what subjects thought about the two categories - whether they, for example, liked the concept of an 'underestimator' for whatever reasons more than that of an 'overestimator'. Even if one could expect that on average this should not play a role, more control can be reached when it is possible to know the evaluative content of the categories. Artificial categories were used again, but a stereotype about the category members was induced, following the mechanism proposed by Fiedler (e.g., Fiedler et al. 1999a; see also section 2.3.1).

In the first two experiments, a preference for one or the other category of new members was financially inconsequential. To test whether preferences were really important for the subjects, they were asked in the computerized experiments to pay for their preference (see also section 4.2). There was thus an immediate cost linked with discriminatory behavior based on group membership. A 'rational', profit maximizing employer should therefore not be willing to pay for this preference, if he would not believe that there was a productivity difference between the two groups large enough to compensate for the cost of 'discriminating'.

\subsubsection{Experiment 3}

Subjects

80 students (54 males, 26 females) from various faculties of the University of Amsterdam participated in teams of four members in four sessions of five teams each.

\section{Material}

The two experiments were computerized using the software z-Tree (Fischbacher, 1999) and were conducted in the experimental laboratory of CREED at the University of 
Amsterdam. All instructions of the experiment were given on paper, but all tasks were done using the computer. The Dutch instructions are provided in appendix III. Division into categories (blue and red) was done by random assignment of subjects to computers. The real-effort task consisted of multiplying two numbers randomly generated by the computer as fast as possible.

\section{Procedure}

Upon arrival, subjects got randomly assigned to a computer, which defined their category - blue or red - and got the first instructions. The first part of the experiment consisted of a stereotype-generation task designed following Fiedler et al. (1999a, b). Subjects had to practice the real-effort task they had to do later (the multiplications), and then got selective feedback about the performance of members of the two categories: From all results of the practice period some were selected and presented sequentially on the screen, such that for the blue category 16 instances of good and eight instances of poor performance were shown, compared to eight instances of good and four instances of poor performance for the red category. Fiedler et al. (1999a, b) show how such a distribution can lead to the impression that the blue category performs better (see section 2.3.1). In essence, subjects thus got information about the two categories, but with respect to mean performance this information was the same for both categories. Thus, if, as Fiedler et al. (1999a, b) show, subjects really develop the stereotype that the category which they got more information about performs better, this is a 'wrong' stereotype, which 'rationally' should not play a role in decision-making. The information was presented on the screen in the following way: "Calculation number three: someone with the category blue did this correctly" or "Calculation number five: someone with the category red did this wrongly".

After this stereotype-generation task, subjects were distributed into three homogeneous 'blue' teams and two 'spare' teams (one 'red'-only team and one mixed team), which were necessary for the discrimination measure, as in the experiments before. The difference with the first experiments is that now some of the members of the 'spare' teams were really 'added' to the old teams following the preferences indicated, and three more rounds were played. The remaining members of the 'spare' teams played the last rounds in one extra team.

Teams first played two rounds of the real-effort game. In the strong-competition treatment, they were told that 'output prices', i.e., the money they got for each correct 
multiplication made, varied between 0.75 and 1.25 guilders $(€ 0.34-€ 0.57)$. In the weak competition treatment, these output prices were said to vary between 1.25 and 1.75 guilders ( $€ 0.57-€ 0.79)$. Output prices were given as a range to enhance uncertainty, but still give subjects some information so that they could develop expectations. Further, this models a situation of competition with imperfect information a bit more closely. Teams had one minute for 'production' in each round. Each team started with a minus of 4 guilders ( $(\ell 1.82)$, as each team-member costed 1 guilder $(\ell 0.45)$ per round. This was supposed to represent costs a firm incurs for wages. Any gains teams made were distributed equally among all members. Subjects got feedback on group performance of the own group (not on output prices) after each round.

After two rounds, members of the blue teams were asked to choose a new member for their team, which they would get from the spare teams. They could indicate (the strength of) their preference for either a red or a blue new member using a scrollbar. Those who had indicated a preference were then asked how much they were willing to pay to enhance the probability of getting the preferred member. The initial probability was said to be 50:50 for a new member of either category. Subjects could choose to pay $15 \%, 30 \%, 45 \%, 60 \%$ or $75 \%$ of the output price of the last round to raise the probability of hiring the preferred member by successive steps of $10 \%$. Thus, by paying $75 \%$ they got the preferred member for sure (see table 4.1 in section 4.2). The four-person team together had to make this payment, not the individual subject, which means that each subject privately only had to pay one fourth of the money.

After all subjects had made their decisions, the computer randomly chose one team member of each team whose decision was implemented. New teams were formed and payments were made if applicable. Three more rounds were played in the new teams. After the fifth round the experiment was concluded with a questionnaire, asking about age, sex and subjective evaluations of each category. Subjects were asked which category they think performed better on the task. The same kind of scrollbar was used as for measuring the preference for a new member. Furthermore, identification with the own category was measured using one question ("How strongly did you identify with your category (blue/red)?"), and uncertainty was measured by one question as well ("How uncertain did you feel with respect to your results in this experiment?"). Both questions could be answered using vertical scroll-bars ranging from zero to four. Experiment 3 thus used different, shortened, questions compared to experiments 1 and 2 to measure identification and uncertainty. This was done because in experiment 3 (and 4) these 
psychological 'background'-relationships no longer were the main focus of interest. The psychological variables were measured at the end of the experiment, to avoid that making these factors conscious would influence behavior, but thereby not allowing for clear causal inferences with respect to discriminatory behavior.

Finally, subjects were paid individually, based on their performance. There was no show-up fee paid, but subjects could expect from the announcements to earn something between 15 and 30 Dutch Guilders ( $€ 8$ and $€ 15$ in experiment 4 ). They further knew that CREED usually pays at least a small amount of money for participation, if no earnings are made. Of course, it was obvious that in the strongcompetition treatment more effort had to be exhibited by the team members to make decent earnings than in the weak-competition treatment.

\section{Results}

Experiment three was analyzed using aggregate data on a team basis, as members of one team got the same feedback and thus were not independent. All teams could be included in the analysis. As the sample still was rather small, non-parametric ManWhitney- $U$ tests were used for comparisons between treatments. With respect to significance testing the same applies as for the first two experiments. If not indicated otherwise, significance tests for correlations were performed 1-tailed, following the specification of the hypotheses. In the following, we refer to SCT when talking about the strong-competition (or low-output-price) treatment and to WCT when talking about the weak-competition (or high-output-price) treatment. Descriptive statistics of the results are given in the following table:

\begin{tabular}{|c|c|c|c|c|c|c|}
\hline \multirow{2}{*}{$\frac{\text { WCT }}{\text { WCT }}$} & & $\mathrm{N}$ & Minimum & laximum & Mean & Std. Dev. \\
\hline & $\begin{array}{r}\text { Preference for new } \\
\text { member }\end{array}$ & 6 & 29.00 & 62.75 & 43.33 & 11.81 \\
\hline & Payment & 6 & .00 & 11.25 & 3.75 & 4.11 \\
\hline & Evaluation of category & 6 & 17.75 & 68.25 & 41.04 & 16.20 \\
\hline & Identification & 6 & 25 & 1.50 & 1.00 & .45 \\
\hline & Uncertainty & 6 & 1.25 & 3.00 & 2.08 & .56 \\
\hline SCT & $\begin{array}{r}\text { Preference for new } \\
\text { member }\end{array}$ & 6 & 38.00 & 73.25 & 54.79 & 13.05 \\
\hline & Payment & 6 & 3.75 & 15.00 & 6.88 & 4.98 \\
\hline & Evaluation of category & 6 & 26.25 & 61.25 & 45.04 & 13.51 \\
\hline & Identification & 6 & .75 & 2.50 & 1.46 & .66 \\
\hline & Uncertainty & 6 & 75 & 2.25 & 1.50 & 50 \\
\hline
\end{tabular}

Table 4.4: Descriptive statistics 
Hypothesis 1) (relationship a) in figure 4.2): There is no significant difference in perceived uncertainty between the two treatments $(\mathrm{Z}=-1.62$, as. sig. $=.105$ (2-tailed)). The test was performed 2-tailed after the means already showed that the tendency goes in the opposite direction from what was expected, namely higher uncertainty in WCT than in SCT.

Hypothesis 2): (relationship b) in figure 4.2): Identification does not differ significantly between the two treatments $(\mathrm{Z}=-.98$, as. sig. $=.163$ (1-tailed)). Perceived uncertainty correlates significantly negative with identification, i.e., the stronger someone identifies the more certain this person is $(r=-.83, p=.001$ (2-tailed)).

Hypothesis 3) (relationship c) and e) in figure 4.2): There is in general no significant difference in preferences between the treatments $(Z=-1.44$, as. sig. $=.150$ (2tailed)). Still, there is a difference between treatments with respect to which preferences become significantly different from zero: Preference for a blue new member differs significantly from zero in WCT, but only marginally significantly in SCT (WCT: $t=$ $2.62, p=.023 ;$ SCT: $\left.t=1.51, p=.095(1 \text {-tailed })^{61}\right)$. Preference for a red new member, on the other hand, differs significantly from zero in SCT, but not in WCT (WCT: $t=1.0, p$ $=.182 ;$ SCT: $\mathrm{t}=2.17, \mathrm{p}=.042(1$-tailed) $)$.

Hypotheses 4) (esp. relationships c) d) and e) in figure 4.2): There is no significant difference in payment between the treatments $(Z=-1.35$, as. $s i g .=.178(2-$ tailed)), but after excluding one outlier ${ }^{62}$, the difference becomes marginally significant, i.e., significant on the $10 \%$ level $(Z=-1.91$, as. sig. $=.056$ (2-tailed)). Subjects are willing to pay slightly more in SCT than in WCT. If payment is split by whether subjects want to pay for a red or a blue new member, there is a marginally significant difference in payment for a red new member between the two treatments, payment being higher in SCT (for red: $Z=-1.80$, as. sig. $=.072$; for blue: $Z=-.73$, as. sig. $=.465$ (2-tailed)).

The total amount of payment (i.e., payment for blue plus payment for red category members together) does differ significantly from zero in both treatments, and more so in SCT (WCT: $\mathrm{t}=2.45, \mathrm{p}=.035$; $\mathrm{SCT} \mathrm{t}=3.38, \mathrm{p}=.01$ (both 1-tailed as the testvalue is zero and values below zero are impossible). Payments for both categories

\footnotetext{
${ }^{61}$ This test was performed 1-tailed as values below zero were not possible. This holds for all similar tests of difference from zero.

${ }^{62}$ Outliers were identified following the standard definition given for boxplot diagrams in SPSS as values between $1.5-3$ box length away from the median. The box length corresponds to the interquartile range, i.e., the difference between the $75^{\mathrm{t}}$ and $25^{\mathrm{th}}$ percentiles.
} 
separately differ also significantly from zero (payment for red: $t=2.16, p=.025$; for blue $t=2.24, p=.02$ (1-tailed)). Split by treatment, payment for a blue new member differs marginally significantly from zero in both treatments, whereas payment for red does not differ from zero in WCT, but it significantly does so in SCT (WCT: Payment for red: $t=$ $1.0, p=.187 ;$ for blue $t=1.75, p=.070 ; S C T:$ For red $t=2.24, p=.035 ;$ for blue $t=$ $1.58, p=.085$ (all 1-tailed)). Table 4.5 shows descriptive statistics and U-tests for these results.

\begin{tabular}{crrrcc}
\hline Treatment & N & Mean & Std. Dev. & Std. Err. Mean \\
\hline WCT & Preference for blue new member 6 & 8.79 & 8.23 & 3.36 \\
& Preference for red new member 6 & 2.13 & 5.21 & 2.13 \\
& Strength of preference in general 6 & 10.92 & 7.07 & 2.88 \\
& Payment for red new member 6 & .63 & 1.53 & .63 \\
& Payment for blue new member 6 & 3.13 & 4.38 & 1.79 \\
SCT & Preference for blue new member 6 & 3.21 & 5.19 & 2.12 \\
& Preference for red new member 6 & 8.00 & 9.05 & 3.69 \\
& Strength of preference in general 6 & 11.21 & 6.87 & 2.81 \\
& Payment for red new member 6 & 5.63 & 6.16 & 2.52 \\
& Payment for blue new member 6 & 1.25 & 1.94 & .79 \\
\hline
\end{tabular}

\begin{tabular}{cccccc}
\hline & $\begin{array}{c}\text { Strength of } \\
\text { preference }\end{array}$ & $\begin{array}{c}\text { Preference } \\
\text { for blue }\end{array}$ & $\begin{array}{c}\text { Preference } \\
\text { for red }\end{array}$ & $\begin{array}{c}\text { Payment } \\
\text { for red }\end{array}$ & $\begin{array}{c}\text { Payment for } \\
\text { blue }\end{array}$ \\
\hline Mann-Whitney U & 15.00 & 10.00 & 10.00 & 8.00 & 14.00 \\
Z & -.48 & -1.37 & -1.43 & -1.80 & -.73 \\
$\begin{array}{c}\text { Asymp. Sig. (2- } \\
\text { tailed) }\end{array}$ & .630 & .171 & .153 & .072 & .465 \\
\hline
\end{tabular}

Table 4.5: Descriptive statistics of the variables analyzed and Mann-Whitney-U tests for differences between treatments in these variables.

To relate the results to the model described in 4.2 , it must be noted that the variable 'payment' here represents $\widetilde{D} / P$ and not $D^{*}$. Differences in 'payment' therefore do not necessarily mean that $D^{*}$ has become larger, because P differs between the two treatments, such that it is on average smaller in SCT than in WCT. 'Preference' on the other hand represents $D U$, i.e., the stronger the preference for any of the categories, the stronger the perceived DU of participants can be assumed to be.

Further exploratory analyses 
Relationship of preference and payment for a certain category with performance evaluation: To test the underlying assumptions of hypotheses 3 and 4 , one has to look at the subjective evaluation of the performance of the categories as measured in the questionnaire - even if it has been measured only at the end of the experiment, it might give an indication of (the influence of) performance stereotypes. A significant correlation between evaluation of the categories and preference $(r=.65, p=.012)$ was found. When analzyed separately for WCT and SCT however, this holds only in WCT, which is rather surprising (WCT: $\mathrm{r}=.90, \mathrm{p}=.016 ; \mathrm{SCT} \mathrm{r}=.41, \mathrm{p}=.416$ ).

Payment for a red new member correlates significantly with the evaluation of red category members $(r=.572, p=.026$ (1-tailed)). Evaluation of blue category members does not correlate with payment for a blue new member. Analysed for WCT and SCT separately, payment for a red new member continues to correlate significantly with evaluation in WCT and marginally significantly in SCT. Payment for a blue new member does not correlate significantly with evaluation of blue category members in any treatment $(\mathrm{WCT}-$ red: $\mathrm{r}=.82, \mathrm{p}=.022$; blue: $\mathrm{r}=-.21, \mathrm{p}=.343 ; \mathrm{SCT}-$ red: $\mathrm{r}=.70, \mathrm{p}=$ .061 ; blue $\mathrm{r}=-.22, \mathrm{p}=.340$ ).

Relationship of performance evaluation of the two categories with other relevant variables: Overall, evaluation of the blue category is significantly different from zero, while evaluation of the red category is only marginally significantly different from zero (for blue $\mathrm{t}=3.30, \mathrm{p}=.004$; for red $\mathrm{t}=1.71, \mathrm{p}=.058$ (both 1-tailed); see table 4.6). This could be seen as a manipulation check, but as the evaluation has been measured at the end of the experiment, it is influenced by everything that happened during the experiment and thus, does not constitute a valid manipulation-check.

\begin{tabular}{ccccc}
\hline & N & Mean & Std. Dev. & Std. Err. Mean \\
\hline Evaluation of blue & 12 & 9.9 & 10.38 & 3.00 \\
Evaluation of red 12 & 2.94 & 5.95 & 1.72
\end{tabular}

\section{Table 4.6: Descriptive statistics of evaluations of the two categories}

Evaluation of a blue new member correlates marginally significantly with identification with the (blue) ingroup $(r=.48, p=.056)$, but not with payment for blue.

Evaluation of the performance of both categories correlates significantly with the feedback the group got in the round before the decision was made $(r=.65, p=.021)$, i.e., the better the feedback, the more subjects thought that blue new members performed better than red new members and vice versa. This feedback also correlates marginally 
significantly with preference $(r=.57, p=.053)$, i.e., the worse the group did, the stronger is the preference for a red (i.e., outgroup) new member.

\section{Discussion}

For an interpretation of the results several problems have to be taken into consideration. First, data on the evaluation of the two categories, subjective uncertainty, and identification with the category were collected at the end of the experiment, to avoid any influence of making these factors conscious before making the 'hiring' decision. Thus, it is unknown how people scored on these variables at the time of making the decision, and in how far these variables, at the time of measurement, were in turn rather influenced by the decision made before. This could be the case either in the sense of justifying the decision or being influenced by the experience with the new member.

Secondly, feedback about performance of the own team was given after each round, and this influenced the evaluation of the categories and preferences, even if the feedback was absolute and not relative, i.e., subjects did not know whether their team scored good or bad in comparison to other teams. Subjects also did not know exactly how much money they earned, because they were only told the range output prices could be in. This allowed them to form expectations, but uncertainty (risk) about the money earned existed until the end of the experiment.

Thirdly, it has not been checked directly whether stereotype induction in the beginning had worked, to avoid making conscious that there might be a difference in performance between the two categories, i.e., to avoid demand effects ${ }^{63}$, before subjects made the 'hiring' decision. Evaluation of the two categories asked about at the end of the experiment did, however, differ significantly from indifference for the blue category and only marginally significantly for the red category. This could indicate that stereotype induction has worked, but it does not necessarily so because during the game feedback about the performance of the teams was given. This feedback did influence preferences and could also have influenced the evaluation measure, especially after experience with 'old' blue and new red members.

Lastly, as data had to be analyzed aggregated by group, the number of independent observations per treatment was quite low. Therefore, non-parametric

\footnotetext{
63 'Demand effects' means that there are (implicit) cues in the experimental design such that subjects get to know the hypothesis and then act like this.
} 
methods had to be used, making it more difficult to detect existing significant effects. These caveats have to be kept in mind as qualifiers for the intepretation of the results.

Overall, the results show a quite complex pattern. The first hypotheses could not be confirmed. It was confirmed that perceived uncertainty is related to identification, but, in contrast to experiment 1 , the more certain subjects were, the stronger they identified with their ingroup. An explanation for this could be that in experiment 1 there was a time lag between measurement of uncertainty and identification, such that those who were uncertain in the beginning could identify with their category to reduce felt uncertainty $(\Psi)$. In experiment 3 on the contrary, perceived uncertainty and identification were measured at the same time, at the end of the experiment, such that those who had identified strongest thought of themselves also to be very certain.

In the strong-competition treatment, outgroup bias was found, while in WCT there was also ingroup bias (with respect to preferences), such that hypothesis 3 could overall not be confirmed. Hypothesis 4 could be confirmed in some respect: There was more payment in SCT than in WCT, but this payment was not necessarily made for ingroup members.

Evaluation of the categories influenced preferences only in WCT. Payment for red new members (outgroup) correlated with a positive evaluation of red category members. Payment for blue new members, however, did not correlate with evalation of blue category members, and was also not often made. This means, subjects were willing to pay more for the group they thought to perform better, but this was not the ingroup, contrary to what was expected.

The outgroup bias found in SCT can be explained psychologically following Spears et al. (2001): Groups feeling lower in status or performance have often been found to favor the outgroup, especially if they do not identify very strongly with their category - which was the case in the experiment reported here. Identification was in general quite low - measured on a scale from 0 to 4 , the mean was 1.23 (std. dev. .59). Spears et al.'s explanation fits also well with the finding that the feedback the groups got about team performance did influence their preferences: Outgroup bias was stronger when subjects got more negative feedback about performance of their own group, i.e., when they felt to perform worse - even if, as mentioned before, feedback was absolute, not relative. However, if there is no reason to assume a strong influence of prejudice, and the stereotype of the ingroup is negative due to feedback, outgroup bias can simply be explained as economically rational behavior in this situation. 
Obviously, in the more competitive situation, people relied on the information about performance of the categories they inferred from feedback, resulting in outgroup bias. This did not happen in the low-competition treatment. However, in WCT, and partially also in SCT, there was also ingroup bias with respect to preference for the blue category. (Positive) evaluation of the blue category also correlated with identification with this category. However, this did hardly result in payment for new members of this category, which is a good illustration of the proposition that stated (ingroup) preferences do not always translate into behavior.

Experiment 3 provided evidence for an interplay of economic and psychological factors. The role of economic factors became visible in that under strong competition all information, which could be seen as diagnostic, was used 'rationally'. If subjects believed that categories contained productivity-related information, this could, as in experiment 2, be interpreted as weak statistical discrimination, as there were no real productivity differences between the categories. As in experiment 2, this effect appeared only when competition was strong. Of course, subjects in the strong-competition treatment had the same database as those in the weak-competition treatment, i.e., category information was not more informative than in the low-competition treatment, but obviously it was taken as more informative. This is evidence for the psychological mechanism assumed. Correlational analyses could confirm only some aspects of the psychological reasoning about underlying processes, but as the information about uncertainty, identification and evaluation of the performance of the categories was collected at the end of the experiment, these results should be interpreted cautiously. Taken together, this is additional evidence that weak statistical discrimination based on (wrong) beliefs about category differences strengthens when competition strengthens. Interesting is that payment was nearly only found in relation to outgroup bias. This suggests that subjects showing ingroup bias in their preferences are more aware of the 'noneconomic' motivations they might have for preferring one category over the other.

A second computerized experiment was conducted without giving group feedback. That way, the influence of feedback on the perception of the categories could be avoided, and more independent data were available, as data now did not have to be analyzed aggregated by teams. Furthermore, by pointing participants explicitly to the number of red and blue members in the game (enabling them to relate the pieces of information to group size, if they wanted), it was made easier for them to detect that the information was in general the same for both groups. That way, subjects should be more 
aware of the fact that category membership is not informative for productivity. This was done to avoid a kind of demand effect: If subjects held wrong beliefs about the difference in numbers of 'red' and 'blue' subjects, they could assume that there is a difference in the amount of information they have about each of the two groups. Preferences would then not result from a (wrong) stereotype, but from rational economic behavior to favor the group about which more information is available. Imagine, for example, that subjects assume that there are less blue than red subjects. In that case, the information they get would imply that they have more instances of information about each blue subject than about each red subject, which implies that they should rationally favor the blue category members.

\subsubsection{Experiment 4}

\section{Subjects}

72 students from various faculties of the University of Amsterdam (47 males, 25 females) participated in four sessions of 20 res. 12 persons each.

\section{Material}

Basically the same material as in experiment 3 was used. Final questions were the same with one exception: To get a more differentiated measure of uncertainty, a threeitem scale was used ("When you played the game, how well did you understand what happened in this situation?"; "When you played the game, how uncertain have you been about what happened in this situation?"; "When you played the game, how well could you predict what would happen?"). This was supposed to measure uncertainty (C) about several aspects of the situation, together capturing a broader concept of uncertainty as the question in experiment 3 , but still implying uncertainty about the result. Such uncertainty gets (of course not very strong) self-relevance from the fact that understanding and being able to predict the situation is related to earnings in that situation. The experiments also aimed at developing valid measurements of the variables in question, therefore we changed the uncertainty-measurement several times.

\section{Procedure}

The two computerized experiments differed mainly with respect to the feedback subjects got after each round: In experiment 4 there was no feedback at all. A further difference was that participants were explicitly pointed to the number of blue/red category-members in the game, as described before. 


\section{Results}

Unfortunately, for one session only twelve participants showed up. But, there were no significant differences in any of the relevant variables between participants in this session and those in the comparable 20-participants session. The session is therefore included in the analysis. Some participants indicated in the final questionnaire that they already participated in similar experiments, but as their data did not significantly differ from the other data in any of the relevant variables, it was included in the analysis, too.

This time data was analyzed on an individual basis, because there was no feedback given to the teams during the experiment. Table 4.7 shows the descriptive statistics of the most important variables for both treatments.

\begin{tabular}{|c|c|c|c|c|c|}
\hline treatment & $\mathrm{N}$ & Minimum & Maximun & $n$ Mean & Std. Dev \\
\hline \multirow[t]{5}{*}{ SCT } & Preference for new member 24 & 0 & 100 & 51.75 & 29.92 \\
\hline & Payment 24 & $\mathbf{0}$ & 45 & 6.25 & 11.63 \\
\hline & Identification 24 & 0 & 4 & 1.13 & 1.23 \\
\hline & Evaluation of category 24 & 9 & 100 & 49.79 & 22.37 \\
\hline & Uncertainty 24 & .67 & 4.00 & 2.11 & 92 \\
\hline \multirow[t]{5}{*}{ WCT } & Preference for new member 20 & 4 & 100 & 58.75 & 25.36 \\
\hline & Payment 20 & 0 & 15 & 1.50 & 4.62 \\
\hline & Identification $\mathbf{2 0}$ & 0 & 4 & 1.30 & 1.34 \\
\hline & Evaluation of category 20 & 11 & 100 & 51.95 & 23.24 \\
\hline & Uncertainty 20 & 67 & 4.00 & 2.30 & 90 \\
\hline
\end{tabular}

Table 4.7: Descriptive Statistics

Hypothesis 1): (relationship a) in figure 4.2): The three items which were used to measure uncertainty did not form a very reliable scale (Cronbach's alpha $=.61$ ), so it was not possible to use them aggregated for the analysis. We did some tests using single items of the scale: There is a significant difference between the treatments with respect to item $1(Z=-1.84$, as. sig. $=.041$ (1-tailed)), i.e., subjects feel more certain with respect to understanding what happened during the game when output-prices are high.

Hypothesis 2): (relationship b) in figure 4.2): Identification does not differ significantly between the two treatments $(\mathrm{Z}=-.45$, as. $\mathrm{sig}=.644$ (2-tailed)), nor does it correlate significantly with any of the uncertainty items.

Hypothesis 3). (relationships c) and e) in figure 4.2): Overall, preferences do not differ between SCT and WCT $(\mathrm{Z}=-1.359$, as. sig. $=.174$ (2-tailed)).

Contrary to the results of experiment 3 , in WCT only preference for red differs significantly from zero $(t=2.886, p=.011)$, whereas in SCT, preferences for both categories differ significantly from zero $(\mathrm{red}: \mathrm{t}=2.922, \mathrm{p}=.011$; blue $\mathrm{t}=2.882, \mathrm{p}=.011$ 
(all 1-tailed) $^{64}$ ). Differences in preference for blue and in preference for red between the two treatments do not become significant (for preference for red: $Z=-.756$, as. sig. $=.45$; for preference for blue $\mathrm{Z}=.44$, as. sig. $=.66$ (2-tailed); see table 4.8 for descriptive statistics).

\begin{tabular}{ccccc}
\hline & $\mathrm{N}$ & Mean & Std. Deviation & Std. Error Mean \\
\hline Evaluation of blue & 44 & 7.23 & 11.99 & 1.81 \\
Evaluation of red 44 & 8.00 & 15.66 & 2.36
\end{tabular}

Table 4.8: Descriptive statistics of evaluations of the two categories

Hypothesis 4) (esp. relationships h), d) and e) in figure 4.2): As expected after experiment 3 , the willingness to pay for a preference is marginally significantly stronger in SCT than in WCT $(\mathrm{Z}=-1.61$, as. sign. $=.054$ (1-tailed)). Only in SCT, general payment for any of the categories differs significantly from zero $(\mathrm{t}=2.63, \mathrm{p}=.015)$. Split by category, both payment for red and payment for blue new members are significantly different from zero in SCT, but not in WCT (WCT: blue: $t=1.00, p=.17$; red: $\mathrm{t}=1.00, \mathrm{p}=.17 ; \mathrm{SCT}$ : blue: $\mathrm{t}=1.70, \mathrm{p}=.052 ;$ red: $\mathrm{t}=1.81, \mathrm{p}=.042$ (all 1-tailed)). More in general, those who prefer a red new member are more willing to pay for their preference than those who prefer a blue new member (overall: $r=.28, p=.032$ ) - at least in the low-price treatment (SCT: $r=.43, p=.018$ ); see table 4.9 for descriptive statistics and U-tests).

\begin{tabular}{crrrc}
\hline Treatment & N & Mean & Std. Dev. & Std. Err. Mean \\
\hline Low output prices & Preference for red 24 & 11.04 & 19.97 & 4.08 \\
& Preference for blue 24 & 9.29 & 16.79 & 3.43 \\
& Payment for blue 24 & 2.50 & 7.22 & 1.47 \\
High output prices & Payment for red 24 & 3.75 & 10.13 & 2.07 \\
& Preference for red 20 & 12.55 & 20.37 & 4.55 \\
& Preference for blue 20 & 3.80 & 11.31 & 2.53 \\
& Payment for blue 20 & .75 & 3.35 & .75 \\
& Payment for red 20 & .75 & 3.35 & .75 \\
\hline
\end{tabular}

\begin{tabular}{ccccc}
\hline & Pref. for red & Pref. for blue & Paym. for blue & Paym. for red \\
\hline Mann-Whitney U & 191.00 & 202.50 & 171.00 & 171.50 \\
Z & -1.30 & -1.13 & -1.01 & -1.32 \\
Asymp. Sig. (2-tailed) & .193 & .260 & .310 & .186 \\
\hline
\end{tabular}

Table 4.9: Descriptive statistics and Mann-Whitney-U test for differences between treatments

${ }^{64}$ Again, values below zero were not possible. The same holds for payment (below). 
Further exploratory analyes:

Relationship of preferences and payment with the evaluation of the categories: Overall, preferences are strongly correlated with the evaluation that a certain category performed better on the task $(r=.77, p=.000$ ). Both the (positive) evaluation of the red and the blue category separately differ significantly from zero (blue: $t=4.00, p=.000$; red: $\mathrm{t}=3.39, \mathrm{p}=.001$ (both 1-tailed)).

(Positive) evaluation of both categories is (marginally) significantly correlated with payment for each category (blue: $\mathrm{r}=-.24, \mathrm{p}=.056$; red: $\mathrm{r}=.26, \mathrm{p}=.042$ (1-tailed)). Split by treatment, these correlations remain significant in SCT, but hardly in WCT: Only one subject in WCT wants to pay for a blue new member, and only few for red new members (SCT: blue: $\mathrm{r}=-.34, \mathrm{p}=.050$; red: $\mathrm{r}=.49, \mathrm{p}=.007$; WCT: blue: $\mathrm{r}=-.05$, $\mathrm{p}=$ .413 ; red: $\mathrm{r}=-.32, \mathrm{p}=.082)(1-$ tailed);

Relationship of evaluation of the categories with other variables: Evaluation of the blue category overall is marginally significantly correlated with identification $(r=$ $.23, \mathrm{p}=.066$ (1-tailed)). This correlation is significant on the $5 \%$ level in SCT $(r=.37$, p $=.038$ (1-tailed)), and disappears in WCT. Evaluation of the red category does not correlate with identification, neither overall, nor in any of the treatments separately.

Relationship of payment with identification:Over both treatments, the stronger subjects identify with their category, the more willing they are to pay for their preference $(\mathrm{r}=.34, \mathrm{p}=.024)$. Identification correlates marginally significant with payment for a red new member $(\mathrm{r}=.30, \mathrm{p}=.065)$.

Sex differences: We also tested for sex differences, which in fact existed: In general, women pay more for their preferences $(\mathrm{Z}=-3.04$, as. sig. $=.002)$, and they are also stronger identifying $(\mathrm{Z}=-2.21, \mathrm{p}=.027$ (both 2-tailed)).

\section{Discussion}

In the fourth experiment some results from the third experiment could be replicated. Again, when competition was stronger, more payments for getting the preferred member were made, and preferences tended towards outgroup bias. However, as there was no feedback given and the belief that the ingroup was performing badly could not develop that easily, there was more ingroup bias with respect to stated preferences in SCT than in experiment 3. Contrary to experiment 3, in WCT only outgroup bias with respect to preferences was found. In WCT hardly any payment was 
done, and payment for blue new members was not significantly different from zero in any of the conditions. Hypothesis 2 could not be confirmed, but again payment correlated positively with identification, and especially payment for preferred red new members. Identification, however, also correlated with more positive evaluations of the performance of the blue category, as measured at the end of the experiment. Evaluations of the categories did correlate with preferences, and this time in both treatments with both preferences. Especially when competition was strong, new members of the category, which was seen as performing better, were preferred - even if categories were not informative about performance in fact. However, again the preferred category was not necessarily the ingroup. In SCT evaluation of the category correlated with payment. Hypothesis 3 and 4 again are not confirmed - but, more payments are made for the group perceived as performing better. This, however, is not always the ingroup.

Overall, the results are again evidence for a combination of psychological and economic factors playing a role in the determination of discriminatory preferences and behavior in a similar way as in experiments 2 and 3 . In this experiment, however, stronger competition did also lead to ingroup bias with respect to preference and payment, also influencing the evaluation of a category. It did, however, when evaluation of the performance of one's own category and the general level of identification were low, also lead to outgroup bias, just like in experiments 2 and 3 . Low opinion about the performance of one's own category could in the last experiment not be derived from feedback on own-group performance, but it could result from the opinion subjects had about their personal performance. Preferences are thus rather based on stereotypes, and, as expected, no evidence for prejudice as an important factor was found.

Subjects under strong competition have strong preferences and are willing to pay for them, and they also choose the group which they believe performs better - at least when asked about the evaluation afterwards. This is related to the psychological mechanism of identification, as Spears et al. (2001) describe it for outgroup bias. Of course, it could also result from dissonance-reduction after having made a decision and paid for it. Whatever the reason, this does hardly happen when competition is weak.

\subsection{General Discussion}

Taken together, the four experiments provide evidence for a combination of psychological and economic mechanisms, as figure 4.3 shows. In all studies, a significant number of subjects uses the - irrelevant - category information as basis for decision- 
making, and more so, when competition is stronger (apart from experiment 1). Subjects in these situations in general also believe that the category they choose for performs better than the other one. This can be interpreted as the psychological mechanism of identification. It leads, in combination with economic rationality, to a weak form of statistical discrimination.

Psychological mechanism:

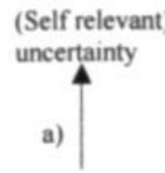

Strong competition

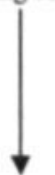

b)

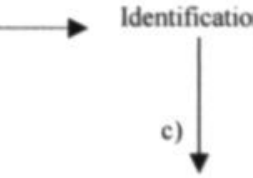

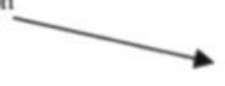

Ingroup bias (prejudice)

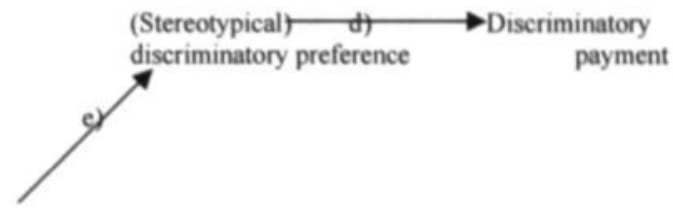

Marginal value of points earned/ risk aversion

'Economic' mechanism:

Figure 4.3: Relationships found between variables - straight lines represent positive relationships found, dashed lines represent ambiguous results.

A general result therefore is that competition makes subjects believe in the informational content of the categories and makes them use it, probably even if consciously (i.e., when asked about the categories) they know that their informational content is very low. However, this only holds when there is at least some reason to believe that categories contain relevant information, i.e., when either categorization is done in a way that relates it to the real-effort task (experiment 2) or when a stereotype has been developed about the categories, which suggests a link of category information and performance (experiments 3 and 4 ). Experiment 1 has shown that when categories cannot be assumed to contain any information about performance, people have weaker preferences when competition is strong, which has been interpreted as them being less risk seeking. In experiment 2 , where categories were seen as a bit more meaningful, subjects under weak competition were still indifferent between them, while preferring the 
outgroup under strong comptition. In experiments 3 and 4 , subjects in weak competition had preferences for one of the categories, as under strong competition, but they were less willing to pay for them, probably because they knew that preferences were based on either just ingroup identification or very unreliable information. This is evidence for a combination of psychological and economic mechanisms: The psychological mechanisms determine that people have preferences at all, but economically rational behavior makes them refrain from paying for 'irrational' preferences - when competition and belief in the informational content of the categories are weak. When competition is strong, psychological mechanisms lead to strengthened belief in the usefulness of the category-information, and 'economic' mechanisms make subjects use this in a 'rational' way. In general, subjects in the experiments are more willing to pay for outgroup preferences, which might result from a kind of self-deception: Subjects may realize when perceiving ingroup bias that this might be a bias, but when they experience outgroup bias, they seem to believe more in its reasonability. However, some strongly identifying subjects especially in experiment 4 also were willing to pay for ingroup preferences.

Another reason for the high levels of outgroup bias found could be the fact that artificial categories were used. Aspects like status of the categories and specific contents of stereotypes of a category, which have a strong influence in real life, did not exist in this experimental setting. Furthermore, only performance sterotypes, and not prejudice about the two categories played a role. The real-world categories labor market discrimination usually applies to are stereotypically rather linked with good than bad ingroup performance. Therefore, psychological ingroup effects will not be counteracted by the 'economic' mechanism linked with negative performance stereotypes of the ingroup. However, minority groups sometimes do show outgroup bias: A real-world example for such a situation is discrimination against female or black employees by female or black employers. When prejudice plays a role, an economic effect of competition can work, and in real-life settings also individual information search is possible - which was not the case in these experiments. It would be important to do similar studies with real categories, and to construct experimental designs where subjects have the possibility to search for individual information in addition to the category information, to see in how far they still rely on category-based information. However, then it will be difficult to prevent individual information from suggesting category information. 
For discrimination in the 'real world', our results allow the inference that when information is imperfect, people will make more use of the information they believe to be contained in a category a person belongs to under strong(er) competition - no matter how relevant the categories really are for an estimation of productivity. If categories contain real productivity-related information, the result will be 'strong' statistical discrimination against individuals. If categories do not contain such information, the result will be group as well as individual discrimination - the weak form of statistical discrimination. However, it is still not clear what influence the 'economic effects' of competition on stereotype use and use of prejudice have in counteracting the psychological mechanisms.

A new aspect with respect to (weak) statistical discrimination is that the experiments have shown that it can become stronger or just appear when competition becomes stronger. Usually, it is assumed that statistical discrimination based on wrong perceptions of productivity differences should disappear when competition gets stronger. The different finding here can be explained by the fact that psychological mechanisms let people rely more on category-based information. The fact that there was hardly any bias at all in the weak-competition treatments in all but the first experiments indicates that categories were too 'empty', but in the real world usually more 'information' is linked with the most salient categories, such that they might even be seen as relevant under weak competition.

Costly discrimination probably happens in the real world under weak competition only when preferences are strong - which, however, probably is the case in most real-life situations. When competition is strong, and when there is a (small) chance that the category provides some information about performance, psychological mechanisms make subjects perceive the cost for 'discriminating' as compensated by the gains that will result from the (perceived) productivity difference. Whether this leads to discrimination against the outgroup or the ingroup depends on beliefs about performance of the ingroup and on beliefs about the requirements of the task. If subjects think that the ingroup performs badly or outgroup members might perform better, they prefer outgroup members.

The experiments showed that outgroup bias is possible, which is rarely discussed in the psychological literature (but, see, e.g., Spears et al., 2001), especially when competition is strong and the ingroup is seen as performing badly. However, preconditions for this to happen in the real world are probably (i) that decision makers assume that their own group performs badly in the task at hand and (ii) that general 
identification with the ingroup is not very high. In the real world, identification with one's own category is stronger than in the laboratory with artificial categories, and this might be even more so when competition is strong. Precondition (i) also often does not hold with respect to real categories, as it is not very probable that e.g. men really might start to think that men in general perform worse than women in a certain, maledominated task. Stressing positive aspects of the outgroup or of diversity might help reducing discriminatory tendencies against outgroups when competition is strong at the same time. It could, however, also be threatening for the ingroup. Such a threat could then lead to stronger identification, or to focusing on other qualities of the own group and in the end, stressing positive aspects of the outgroup might, by these psychological effects, even lead to stronger discrimination. 


\section{Appendix I: Instructions experiment 1}

\section{Categorizing task:}

\section{Point-estimation:}

Dear Participant,

In the following you are asked to estimate the number of points you see at the screen. You have only 6 seconds to look at them, and then 15 seconds to write down your decision. So, do not try to count them, you can't make it! Just give your spontaneous estimation and write it down in the table below! Have fun!

\begin{tabular}{|l|l|}
\hline Slide & Estimated number of points \\
\hline 1 & \\
\hline 2 & \\
\hline 3 & \\
\hline 4 & \\
\hline 5 & \\
\hline 6 & \\
\hline
\end{tabular}

\section{Instruction I}

Dear participant,

Welcome to our team-game!

Thank you for your willingness to participate in this study!

Before we start, I would like to ask you to follow the instructions below during the whole game:

- Please do not talk to each other or otherwise communicate during the studyl

- Please do not try to find out how the others decide or what they answer!

- It is also very important that you follow precisely the instructions - e.g. go on to the next page, if told so, and wait, if told sol

This is very important, otherwise, the study cannot be evaluated properly!

Thank you for your cooperation!

I hope you will have some fun!

\section{Instruction 2:}

Dear Participant,

Thank you for your willingness to take part in our study!

With this study, we want to see how people distribute their effort on different tasks and how this is related to the situation they are in.

You will get one page with two versions of the same kind of task. One represents work for your team, the other work for yourself. For both tasks you get points, depending on how well you do. Your team is 
playing against another team, which is with you here in the room, doing the same task. The outcome of your team thus depends on how both teams do. Your personal outcome depends on how you do, how your team-members do, and how the other team does. This is the main structure of the game. More in detail, the result of the team is figured out as follows:

At the end of each round, the outcomes of the team-task from all members of each team are taken times 2 and added together.

(PR only:)

Your final amount of points in the team-task is calculated relative to the result of the other team.

More precisely, the points achieved by your team will be divided by the total number of points achieved by both teams and then taken times 100 .

The resulting points of all rounds played will constitute your team's final result, which is divided equally among the team members.

The points you achieved in the personal task are added to your final account of points. They are not taken times 21

Example:

Imagine you personally achieved 10 points in the first round for the team-task and 10 points for the private task. Your 3 team-members together reached 33 points on the team-task. The team thus together has 43 points. These are multiplied by 2 , which gives your team 86 points in total.

The other team only reached 35 points, which, multiplied by 2 results in 70 points for the other team.

Together, the two teams have 156 points.

To calculate the resulting points for your team, the 86 points you achieved have to be divided by the 156 points of both teams, which is 0,551 . If one takes this times 100 , the result is 55,1 .

This means, your team gets 55,1 points in the first round, which means 13,77 points for each of the team members.

Additionally, each team member gets its personal points, which in your case are 10. So, your personal result from round 1 is 23,77 points.

\begin{tabular}{|l|l|l|l|l|l|}
\hline & $\begin{array}{l}\text { Team } \\
\text { Task }\end{array}$ & $\begin{array}{l}\text { Private } \\
\text { Task }\end{array}$ & $\begin{array}{l}\text { team points/team } \\
\text { points } * 2\end{array}$ & calculation & $\begin{array}{l}\text { Final outcome per } \\
\text { team-member }\end{array}$ \\
\hline You & 10 & 10 & & & $13,77+10=23,77$ \\
\hline $\begin{array}{l}\text { Your team } \\
\text { members }\end{array}$ & 33 & $\mathrm{X}$ & $43 / 86$ & $(86 / 156)^{*} 100$ & $13,77+\mathrm{X}$ \\
\hline Other team & 35 & $\mathrm{X}$ & $35 / 70$ & $(70 / 156) * 100$ & $11,22+\mathrm{X}$ \\
\hline
\end{tabular}

Before you are divided into your teams, you will do a test round of this kind of task with random teams.

But first you can go on to the next page and have a closer look at the structure of the task.

(WTA only:)

The winning team gets the amount of points achieved, the other team gets nothing. 
The resulting points of all rounds played will constitute your team's final result and are divided equally among all team members.

Your personal points are added to your final account of points.

Example:

Imagine you personally achieved 10 points in the first round for the team-task and 10 points for the private task. Your three team-members together reached 33 points. The team thus together has 43 points. These are multiplied by 2 , which gives your team 86 points in total.

The other team got 35 points on the team-task, multiplied by 2 results in 70 points.

Your team thus won and the 86 points are divided equally among the team members, which means 21,5 points for each of you. Additionally, each team member gets its personal points, which in your case are 10. So, your personal result from round 1 is 31,5 points.

The members of the other team do not get any points from the team-task in this round, but they of course get their personal points.

\begin{tabular}{|l|l|l|l|l|l|}
\hline & $\begin{array}{l}\text { Team } \\
\text { Task }\end{array}$ & $\begin{array}{l}\text { Private } \\
\text { Task }\end{array}$ & $\begin{array}{l}\text { Team points/team } \\
\text { points } * 2\end{array}$ & calculation & $\begin{array}{l}\text { Final outcome per } \\
\text { team-member }\end{array}$ \\
\hline You & 10 & 10 & & & $21,5+10=31,5$ \\
\hline $\begin{array}{l}\text { Your team } \\
\text { members }\end{array}$ & 33 & $\mathrm{X}$ & $43 / 86$ & $86>70$ & $21,5+\mathrm{X}$ \\
\hline Other team & 35 & $\mathrm{X}$ & $35 / 70$ & $86>70$ & $0+\mathrm{X}=\mathrm{X}$ \\
\hline
\end{tabular}

Before you are divided into your teams, you will do a test round of this kind of task with random teams. But first you can go on to the next page and have a closer look at the structure of the task. (Both conditions:)

Here you see how the task is organized: You have one page with both tasks. Above one of them is written "team" and above the other is written "private":

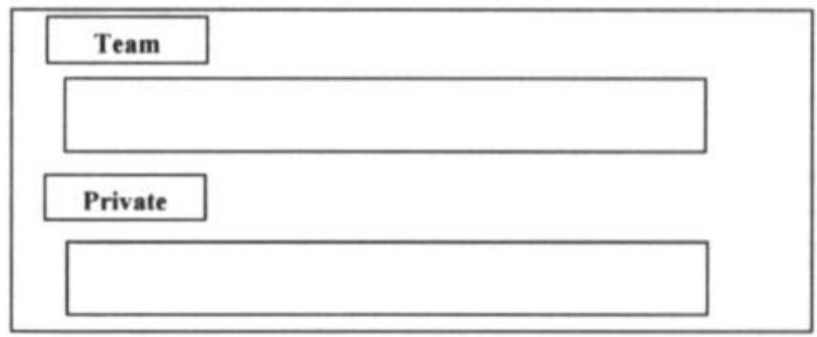

The kind of task is always the same, it will differ in detail in each round and for both tasks, the teamand the private task.

Your can distribute your effort between both tasks, keeping in mind, that only the team-task contributes to the success of your team in the competition with the other team. Points you make in the team-task are taken times 2 and then count for the result of your team, whereas points in the private task are counted 


\section{Experimental test of the model}

once for your personal account. You should work as fast as possible, because you have only two minutes altogether! You won't be able to finish both tasks in this time, but you can work a substantial part on both. You can switch between the two tasks whenever you want.

If you have any questions, please ask now!

The task will be explained now for all of you together, giving an example as well. When you have finished reading this please put these pages face down at the corner of your table and wait until the presentation of the task starts!

\section{Instruction 3:}

After having played the test round now, we ask you to answer two questions on your perception of the game. Please do not think long about the questions, answer spontaneously!

1) How strong is the competition between the teams in your perception? Very strong not strong at all

2) How do you feel about your final result (points) in this game?

Highly uncertain Highly certain

When you have answered these questions, please put the paper face down on your table to signal that you are done.

You will get a paper informing you about your team-members and the form for the second round now. Please do not start with the task for the second round before told sol

For the next round, each team gets one additional member. They have done the practicing task as well, so they know how the game is played. You cannot choose your new team-member, but you can give a preference. The stronger your preference, the closer you make your cross on the line to one of the edges.

The preferences of all team members are analyzed and if there is a clear preference within the whole team, we try to match the new member with this preference - but obviously, we cannot give a guarantee that this will be possible!

Whom would you prefer as additional member in your team?

Strongly prefer

Strongly prefer

Person

Underestimator

Person

Overestimator

When you have indicated your preference, put the page face down at the corner of your table and go on! 
Questionnaire on perception of the situation:

While we check the preferences of the teams, and try to add the new member following your preference, you are asked to answer some questions on your perception of the situation your team and you are in:

A) Please mark your answer by making a cross on the line!

1) How strong is the competition among the teams in your perception?

Very strong not strong at all

2) How do you feel about your final result (points) in this game?

Highly uncertain Highly certain

3) How do you feel about the performance of your team?

Highly uncertain Highly certain

4) How do you feel about the performance of overestimators in this game?

Highly uncertain Highly certain

B) Please make a cross in the box, which represents your feelings best!

1) What do you think, how much you might like other overestimators? not very much

2) How similar do you think you might be to other overestimators in terms of general attitudes and opinions?

\begin{tabular}{|l|l|l|l|l|l|l|l|l|l|l|}
\hline not very much & 1 & 2 & 3 & 4 & 5 & 6 & 7 & 8 & 9 & very much \\
\hline
\end{tabular}

3) How much would you like to get to know other overestimators? not very much

4) How much do you feel to belong to other overestimators?

\begin{tabular}{|l|l|l|l|l|l|l|l|l|l|l|}
\hline not very much & 1 & 2 & 3 & 4 & 5 & 6 & 7 & 8 & 9 & very much \\
\hline
\end{tabular}

5) What do you think, how much you might like other members of your recent team?

\begin{tabular}{|l|l|l|l|l|l|l|l|l|l|l|}
\hline not very much & 1 & 2 & 3 & 4 & 5 & 6 & 7 & 8 & 9 & very much \\
\hline
\end{tabular}

6) How similar do you think you might be to other members of your recent team in terms of general attitudes and opinions?

not very much

\begin{tabular}{l|l}
\hline 1 & 2 \\
\hline
\end{tabular}

\begin{tabular}{l|l}
2 & 3 \\
\hline
\end{tabular}

\begin{tabular}{l|l|l|}
3 & 4 & 5 \\
\hline
\end{tabular}

6

\begin{tabular}{|l|l|}
\hline 7 & 8 \\
\hline
\end{tabular}

\begin{tabular}{|l|l|}
\hline 9 & very much \\
\hline
\end{tabular}


4 Experimental test of the model

7) How much would you like to get to know your recent team?

\begin{tabular}{|l|l|l|l|l|l|l|l|l|l|l|} 
not very much & 1 & 2 & 3 & 4 & 5 & 6 & 7 & 8 & 9 & very muct
\end{tabular}

8) How much do you feel to belong to your recent team?

\begin{tabular}{|l|l|l|l|l|l|l|l|l|l|l|}
\hline not very much & 1 & 2 & 3 & 4 & 5 & 6 & 7 & 8 & 9 & very muct \\
\hline
\end{tabular}

9) To what extent would you prefer to belong to this team or to the other team?

\begin{tabular}{|l|l|l|l|l|l|l|l|l|l|l|}
\hline not very much & 1 & 2 & 3 & 4 & 5 & 6 & 7 & 8 & 9 & very muct \\
\hline
\end{tabular}

10) How much aware are you of the presence of others?

\begin{tabular}{|l|l|l|l|l|l|l|l|l|l|l|}
\hline not very much & 1 & 2 & 3 & 4 & 5 & 6 & 7 & 8 & 9 & very muct \\
\hline
\end{tabular}

11) How much do you feel observed by others?

\begin{tabular}{|l|l|l|l|l|l|l|l|l|l|l|}
\hline not very much & 1 & 2 & 3 & 4 & 5 & 6 & 7 & 8 & 9 & very muct \\
\hline
\end{tabular}

12) How much do you feel observed by the experimenter?

\begin{tabular}{|l|l|l|l|l|l|l|l|l|l|l|}
\hline not very much & 1 & 2 & 3 & 4 & 5 & 6 & 7 & 8 & 9 & very muci \\
\hline
\end{tabular}

Please signal when you are finished by putting these pages face down at the corner of your tablel When everybody has finished, we are ready for the next round!

Team information:

Your team ( ):

You:

No.:

Your team-members:

1)

No:

2)

No:

3)

No:

4)

No: 


\section{Experimental test of the model}

\section{Instruction 4:}

The experiment is finished now! The results of the second round will be published soon! At the end, we would like to ask you to answer some demographical questions:

How old are you?

What is your nationality? Are you male/female ?

Thank you very muchl

If you want any further information, or if you want to get notified if there are new experiments going on, please send an e-mail to

\section{c.schwieren@algec.unimaas.nl}

You will get your payment now and some information about the present study.

Thank you for your participation!

Have a nice day!

\section{Appendix II : Instructions experiment 2}

\section{Instruction I:}

Liebe Teilnehmerinnen, lieber Teilnehmer!

Willkommen zu unserem Teamwettbewerb!

Bevor wir anfangen, mochten wir Euch bitten, das folgende wahrend des ganzen Spiels zu beachten:

Bitte redet und kommuniziert nicht miteinander!

Bitte versucht nicht, herauszufinden wie andere antworten oder sich entscheiden!

Bitte blattert nur dann um, wenn Ihr dazu aufgefordert werdet!

Es ist fur uns sehr wichtig, dass Ihr Euch daran genau haltet, denn sonst konnen wir die Daten nicht auswerten!

Vielen Dank und viel Spass!

\section{Instruction 2:}

Liebe Teilnehmerin, lieber Teilnehmer!

Diese Untersuchung ist Teil eines Projektes das sich damit beschaftigt, wie Menschen in Teams ihre Aufmerksamkeit auf verschiedene Aufgaben verteilen, und wie dies durch die Situation in der sie sich befinden beeinflusst wird.

Im Folgenden wirst Du in ein Team eingeteilt, und mit diesem Team wirst Du im Wettbewerb mit einem anderen Team Aufgaben losen. Jedes Teammitglied bekommt jeweils ein Blatt mit zwei Versionen der gleichen Aufgabenart. Eine Version bringt Punkte fur Dein Team, die andere fur Dich selbst. Du kannst also in jeder Runde entscheiden, ob Du mehr Einsatz fur die Teamaufgabe oder fur die ,private' Aufgabe einbringen willst. 
Dein Team spielt gegen ein anderes Team hier im Raum, das die gleichen Aufgaben zu erfullen hat. Dein personliches Ergebnis hăngt also davon ab, wieviel Punkte Du selbst in beiden Aufgaben erreichst, wieviel Punkte Deine Teammitglieder in der Teamaufgabe erreichen, und wieviel Punkte das gegnerische Team in der Teamaufgabe erreicht.

(PR only:)

Im Detail wird das Ergebnis wie folgt berechnet:

Am Ende jeder Runde werden die Punkte, die die Mitglieder eines Teams in der Teamaufgabe erreicht haben zusammengezahlt und verdoppelt. Danach wird der relative Anteil jedes Teams an allen Punkten, die im Spiel erreicht wurden, ausgerechnet: Die Punkte, die beide Teams erzielt haben, werden addiert. Dann wird die Punktzahl fur jedes einzelne Team durch diese Gesamtpunktzahl geteilt und mit 100 multipliziert. Das Ergebnis dieser Rechnung ist dann die Punktzahl für das jeweilige Team in der jeweiligen Runde.

Am Ende des Spiels werden dann die Punkte, die jedes Team insgesamt erreicht hat, unter den Teammitgliedern gleichmaßig aufgeteilt.

Die Punkte, die Du in der „privaten“ Aufgabe in allen Runden erreicht hast, werden zu Deinem endgaltigen Resultat addiert, allerdings werden sie nicht verdoppelt.

Ein Beispiel:

Stell' Dir vor, Du erzielst in der ersten Runde bei der Teamaufgabe 10 Punkte und genauso viele bei der privaten Aufgabe. Deine 3 Teammitglieder gemeinsam haben 33 Punkte in der Teamaufgabe erreicht. Insgesamt hat das Team also 43 Punkte. Diese werden verdoppelt, was 86 Punkte fur das Team insgesamt ergibt. Das andere Team hat nur 35 Punkte erreicht - multipliziert mit zwei ergibt das 70 Punkte fur das andere Team.

Beide Teams haben insgesamt also 156 Punkte.

Um das Ergebnis für Dein Team zu berechnen, mussen die 86 Punkte Deines Teams durch 156 geteilt werden. Das Ergebnis ist 0,551. Multipliziert mit 100 ergibt das 55,1. D.h., Dein Team bekommt in der ersten Runde 55,1 Punkte. Geteilt durch die Anzahl der Teammitglieder ergeben sich daraus 13,77 Punkte pro Teammitglied.

Zusatzlich bekommt jedes Teammitglied seine „privaten“ Punkte - in Deinem Fall kommen also noch 10 Punkte hinzu. Dein Gesamtergebnis fur diese Runde ist deshalb 22,77 Punkte.

\begin{tabular}{|l|l|l|l|l|l|}
\hline & $\begin{array}{l}\text { Team- } \\
\text { aufgabe }\end{array}$ & $\begin{array}{l}\text { „Private } \\
\text { Aufgabe }\end{array}$ & $\begin{array}{l}\text { Punkte } \\
\text { Team/Punkte team } \\
-2\end{array}$ & Berechnung & $\begin{array}{l}\text { Endergebnis pro } \\
\text { Teammitglied }\end{array}$ \\
\hline Du & 10 & 10 & & & $13,77+10=23,77$ \\
\hline $\begin{array}{l}\text { Deine } \\
\text { Teammitglieder }\end{array}$ & 33 & $\mathrm{X}$ & $43 / 86$ & $(86 / 156)^{*} 100$ & $\begin{array}{l}13,77+\mathrm{X} \\
(\mathrm{X}=\text { Resultat } \\
\text { private* Aufgabe })\end{array}$ \\
\hline Das andere Team & 35 & $\mathrm{X}$ & $35 / 70$ & $(70 / 156)^{*} 100$ & $11,22+\mathrm{X}$ \\
\hline
\end{tabular}


(WTA only:)

Im Detail wird das Ergebnis wie folgt berechnet:

Am Ende jeder Runde werden die Punkte, die die Mitglieder eines Teams in der Teamaufgabe erreicht haben zusammengezahlt und verdoppelt.

Das Team, das gewonnen hat, bekommt die Punkte, die es erzielt hat, das andere Team bekommt keine Punkte.

Am Ende des Spiels werden dann die Punkte, die jedes Team insgesamt erreicht hat, unter den Teammitgliedern gleichmaBig aufgeteilt.

Die Punkte, die Du in der ,.privaten“ Aufgabe in allen Runden erreicht hast, werden zu Deinem endgaltigen Resultat addiert, allerdings werden sie nicht verdoppelt.

Ein Beispiel:

Stell' Dir vor, Du erzielst in der ersten Runde bei der Teamaufgabe 10 Punkte und genauso viele bei der privaten Aufgabe. Deine 3 Teammitglieder gemeinsam haben 33 Punkte in der Teamaufgabe erreicht. Insgesamt hat das Team also 43 Punkte. Diese werden verdoppelt, was 86 Punkte fur das Team insgesamt ergibt. Das andere Team hat nur 35 Punkte erreicht - multipliziert mit zwei ergibt das 70 Punkte fur das andere Team.

Dein Team hat also gewonnen und die 86 Punkte werden gleichmaBig unter den Teammitgliedem aufgeteilt. D.h., jedes Mitglied Deines Teams bekommt 21,5 Punkte.

Zusatzlich bekommt jedes Teammitglied seine ,privaten“ Punkte - in Deinem Fall kommen also noch 10 Punkte hinzu. Dein Gesamtergebnis fur diese Runde ist deshalb 31,5 Punkte.

Die Mitglieder des anderen Teams bekommen in dieser Runde keinerlei Punkte von der Teamaufgabe, aber naturlich bekommen auch sie ihre personlichen Punkte.

\begin{tabular}{|c|c|c|c|c|c|}
\hline & $\begin{array}{l}\text { Team- } \\
\text { aufgabe }\end{array}$ & $\begin{array}{l}\text { "Private" } \\
\text { Aufgabe }\end{array}$ & $\begin{array}{l}\text { Punkter Team } \\
\text { /Punkte Team } \bullet 2\end{array}$ & Berechnung & $\begin{array}{l}\text { Endergebnis pro } \\
\text { Teammitglied }\end{array}$ \\
\hline Du & 10 & 10 & & & $21,5+10=31,5$ \\
\hline $\begin{array}{l}\text { Deine } \\
\text { Teammitglieder }\end{array}$ & 33 & $\mathrm{X}$ & $43 / 86$ & $86>70$ & $\begin{array}{l}21,5+\mathrm{X} \\
\text { (X }=\text { Resultat } \\
\text { private" Aufgabe) }\end{array}$ \\
\hline Das andere Team & 35 & $\mathrm{X}$ & $35 / 70$ & $86>70$ & $0+X=X$ \\
\hline
\end{tabular}

(Both conditions:)

Bevor die Teams eingeteilt werden, werden wir eine Testrunde mit zufallig zusammengestellten Teams spielen. Auf der nachsten Seite kannst Du sehen wie die Aufgabe genau aussieht!

Hier siehst Du, wie die Aufgaben aufgebaut sind: Du bekommst ein Blatt mit beiden Aufgaben. Eine hat die Oberschrift „Team", die andere „Privat". 


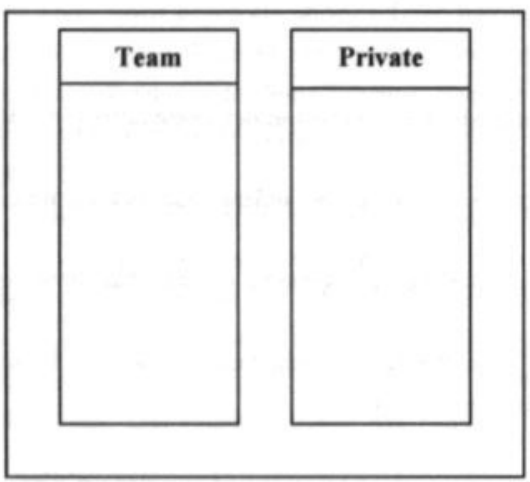

Die Aufgabenart ist immer gleich, die Aufgaben werden sich nur im Detail andern. Die Aufgabentypen werden gleich kurz erklart. Grundsatzlich handelt es sich um „Denkaufgaben“. Du kannst Deinen Einsatz zwischen der privaten und der Teamaufgabe aufteilen. Denk ${ }^{*}$ allerdings dabei daran, dass nur die Teamaufgabe zum Erfolg Deines Teams im Wettbewerb mit dem anderen Team beitrăgt.

Punkte, die Du in der Teamaufgabe machst, werden verdoppelt und für Dein Team gezăhlt, wahrend Punkte in der „privaten“ Aufgabe einfach für Dein persőnliches Resultat zăhlen.

Du musst so schnell wie mogglich arbeiten, denn Du hast insgesamt nur 1 Minute Zeit! Du wirst in dieser Zeit natälich nicht beide Aufgaben fertigstellen konnen, aber Du kannst durchaus bei beiden Aufgaben Punkte machen. Du kannst zwischen den beiden Aufgaben jederzeit wechseln.

Die funf besten Teilnehmer am Teamwettbewerb bekommen einen Kinogutschein!

Wichtig:

Nach einigen Runden wird eine Zwischenbilanz gezogen. Wenn eines der Teams bis dahin weniger als 30 Teampunkte pro Teammitglied erreicht hat, scheidet es aus und das dritte Team im Raum spielt an seiner Stelle mit.

Wenn Du Fragen hast, wende Dich bitte an die Versuchsleiterin!

Wenn Du diese Seiten gelesen und verstanden hast, drehe sie bitte um, damit deutlich sichtbar ist, dass Du fertig bist. Wenn alle fertig gelesen haben wird die Aufgabe genau erklart und eine Testrunde gespielt.

\section{Instruction 3:}

Bevor das Spiel weitergeht, wird jetzt Zwischenbilanz gezogen. Sollte eins der beiden Teams in den ersten drei Runden weniger als 30 Teampunkte erreicht haben, scheidet es aus und das Team, das bisher nicht mitgespielt hat, tritt an seine Stelle (da es ein kleines Team ist, wird fur dieses Team etwas anders gezahlt - aber das wird spater noch ausfuhrlich erklart). Soltten beide Teams mehr als 30 Punkte 
erreicht haben, werden die Mitglieder des "Ersatzteams" auf die bisherigen Teams aufgeteilt. Darum bitten wir Dich, jetzt schon eine Praferenz anzugeben, wen Du als neues Teammitglied mochtest, falls das Ersatzteam aufgeteilt wird.

Wir werden die Praferenzen aller Teammitglieder auswerten, und wenn es eine starke gemeinsame Praferenz im Team gibt, werden wir versuchen, das neue Teammitglied dementsprechend zu wahlen, aber garantieren können wir das naturlich nicht.

Um Deine Praferenz anzugeben, musst Du den Punkt auf der Linie mit einem Strich markieren, der Deiner Praferenz am meisten entspricht.

Wen würdest Du als neues Teammitglied vorziehen?

Starke Praferenz für

kognitiven Stil P

Starke Praferenz fur

kognitiven Stil K

Wenn Du Deine Praferenz angegeben hast, bitte drehe die Seite um, so dass sie eingesammelt werden kann, und fange an, den Fragebogen auszufullen!

\section{Instruction 4}

Wahrend wir Zwischenbilanz ziehen und gegebenenfalls die neuen Mitglieder ihren Teams zuordnen, bitten wir Dich, ein paar Fragen zu Deiner Wahmehmung der Situation zu beantworten:

A) Bitte markiere den Punkt auf der Linie, der Deiner Wahrnehmung am besten entspricht, mit einem Strich!

1) Wie gro $B$ ist Deiner Meinung nach in diesem Spiel die Chance, dass ein Team nach der Zwischenbilanz ausscheiden muss?

Sehr groß

2) Wie groß ist Deiner Meinung nach in diesem Spiel die Chance, dass beide Teams bei der Zwischenbilanz mit ihrem Punktestand (Teampunktel) zufrieden sein konnen?

Sehr groB

3) Wie groß ist Deiner Meinung nach in diesem Spiel die Chance, dass Du personlich bei der Zwischenbilanz mit Deinem Punktestand (Team- und Privatpunkte!) zufrieden sein kannst?

Sehr groß

4) Wie wichtig ist es um in diesem Spiel zu gewinnen, dass alle Teammitglieder ihr Bestes geben fur das Team?

Sehr wichtig

5) Wie stark ist Deiner Meinung nach in diesem Spiel der Wettbewerb zwischen den Teams?

Sehr stark

6) Wie sicher bist Du Dir, dass Du Deine Ziele in diesem Spiel erreichen kannst?

Sehr sicher

Gar nicht sicher 
7) Wie sicher bist Du Dir, dass Dein Einsatz ausreichend war, damit das Team nach der Zwischenbilanz weiterspielen kann?

Sehr sicher Gar nicht sicher

8) Wie sicher bist Du Dir, dass die anderen Mitglieder Deines Teams genug Einsatz erbracht haben, damit das Team weiterspielen kann?

Sehr sicher Gar nicht sicher

9) Wie sicher bist Du Dir, dass Dein Team nach der Zwischenbilanz weiterspielen darf?

Sehr sicher

10) Wie wichtig ist es fur Dich, dass Dein Team nach der Zwischenbilanz weiterspielen darf?

Sehr wichtig

Gar nicht wichtig

B) Mitte mach' ein Kreuz in das Kästchen, das Deine Wahrnehmung am besten wiedergibt!

1) Was glaubst Du, wie sehr Du die anderen $\mathrm{P}$ mögen würdest?

\begin{tabular}{|l|l|l|l|l|l|l|l|l|l|l|}
\hline Uberhaupt nicht gern & 1 & 2 & 3 & 4 & 5 & 6 & 7 & 8 & 9 & Sehr gern \\
\hline
\end{tabular}

2) Was glaubst Du, wie ăhnlich Du den anderen $\mathrm{P}$ in Bezug auf allgemeine Einstellungen und Meinungen bist?

\begin{tabular}{|l|l|l|l|l|l|l|l|l|l|l|}
\hline Uberhaupt nicht ahnlich & 1 & 2 & 3 & 4 & 5 & 6 & 7 & 8 & 9 & Sehr ăhnlich \\
\hline
\end{tabular}

3) Wie sehr hast Du das Gefuhl, dass Du zu den anderen P gehörst?

\begin{tabular}{|l|l|l|l|l|l|l|l|l|l|l|}
\hline Uberhaupt nicht & 1 & 2 & 3 & 4 & 5 & 6 & 7 & 8 & 9 & Sehr stark \\
\hline
\end{tabular}

4) Was glaubst Du, wie sehr Du die anderen Mitglieder Deines Teams mőgen würdest?

\begin{tabular}{|l|l|l|l|l|l|l|l|l|l|l|}
\hline Oberhaupt nicht & 1 & 2 & 3 & 4 & 5 & 6 & 7 & 8 & 9 & Sehr gern \\
\hline
\end{tabular}

5) Was glaubst Du, wie ahnlich Du den anderen Mitgliedern Deines Teams in Bezug auf allgemeine Einstellungen und Meinungen bist?

\begin{tabular}{|l|l|l|l|l|l|l|l|l|l|l|}
\hline Uberhaupt nicht ahnlich & 1 & 2 & 3 & 4 & 5 & 6 & 7 & 8 & 9 & Sehr ahnlich \\
\hline
\end{tabular}

6) Wie sehr hast Du das Gefuhl, dass Du zu Deinem momentanen Team gehörst?

\begin{tabular}{|l|l|l|l|l|l|l|l|l|l|l|}
\hline Oberhaupt nicht & 1 & 2 & 3 & 4 & 5 & 6 & 7 & 8 & 9 & Sehr stark \\
\hline
\end{tabular}

7) In welchem Masse würdest Du lieber zum anderen Team gehören?

\begin{tabular}{|l|l|l|l|l|l|l|l|l|l|l|}
\hline Oberhaupt nicht & 1 & 2 & 3 & 4 & 5 & 6 & 7 & 8 & 9 & Sehr gern \\
\hline
\end{tabular}

8) Wie sehr ist Dir die Gegenwart anderer bewusst?

\begin{tabular}{|l|l|l|l|l|l|l|l|l|l|l|}
\hline Oberhaupt nicht bewusst & 1 & 2 & 3 & 4 & 5 & 6 & 7 & 8 & 9 & Sehr bewusst \\
\hline
\end{tabular}

9) Wie sehr fuhlst Du Dich von anderen beobachtet?

\begin{tabular}{|l|l|l|l|l|l|l|l|l|l|l|}
\hline Oberhaupt nicht & 1 & 2 & 3 & 4 & 5 & 6 & 7 & 8 & 9 & Sehr stark \\
\hline
\end{tabular}

10) Wie sehr fühlst Du Dich von den Versuchsleitern beobachtet?

\begin{tabular}{|l|l|l|l|l|l|l|l|l|l|l|}
\hline Oberhaupt nicht & 1 & 2 & 3 & 4 & 5 & 6 & 7 & 8 & 9 & Sehr stark \\
\hline
\end{tabular}

Bitte drehe diese Seiten um, wenn Du fertig bist, damit wir erkennen kőnnen, wann alle fertig sind.

Dann geht es mit den neuen Teams weiter! 


\section{Appendix III : Instructions experiments $3 \& 4$}

Instruction 1:

Beste deelnemer,

Dit experiment bestaat uit verschillende onderdelen. In dit eerste gedeelte krijg je de kans om de taak te oefenen die je later met je team gaat uitvoeren om geld te verdienen. Verder krijg je informatie over andere deelnemers.

Wat ga je aberhaupt in dit experiment doen? Je wordt later in en groep ingedeeld en dan moet elk groepslid rekensommen maken. Van het aantal goed uitgevoerde rekensommen hangt de teambeloning af, die gelijkmatig onder de teamleden verdeeld word. Wij gaan meerdere ronden spelen. Hoe dat allemaal precies gebeurt vertellen wij je later nog. Nu komt eerst de ronde waarin je kunt oefenen.

De deelnemers in dit experiment zijn willekeurig in verschillende groepen ingedeeld. Er zijn "blauwe" en "rode" groepen en ook nog "oude leden" en "nieuwe leden". In het begin geeft de computer je de informatie over je eigen categorie(en). Je moet verschillende keren tijdens het experiment aangeven, bij welke categorieen je hoort. Dat is belangrijk voor de groepsindeling.

In dit eerste gedeelte nu kun je met het soort rekensommen kennis maken en een beetje oefenen. Je hebt straks 3 minuten de tijd om zoveel mogelijk rekensommen te maken. Als de 3 minuten om zijn, krijgen jullie nog de gelegenheid om je een beeld te vormen hoe de anderen gepresteerd hebben. Pas daarna worden jullie in teams ingedeeld en begint het spel.

Probeer dus zoveel mogelijk berekeningen te doen, en daarvan ook nog zoveel mogelijk goed te maken! Maar realiseer je dat je in de spelperiodes waarin om geld wordt gespeeld voor foute resultaten van de sommen geen strafpunten krijgt.

Je mag geen rekenmachine gebruiken!

Voor het hele experiment is het belangrijk dat je, als je vragen hebt, een teken geeft aan de leider van het experiment. Zij komt dan naar je toe en beantwoordt je vraag. A.u.b. niet met andere deelnemers praten!

En nu veel plezier!

\section{Instruction 2:}

$\mathrm{Nu}$ begint het eigenlijke spel. Jullie worden nu in teams ingedeeld. Elk team representeert een bedrijf dat op een markt moet overleven. Het bedrijf produceert een product (het resultaat van de rekensommen) en verkoopt het voor en prijs, die door de markt bepaald wordt. Het bedrijf (het team) maakt 1 gulden kosten voor elk teamlid, dus een vierpersoonsteam begint met een min van 4 gulden te spelen. Voor de "productie" hebben jullie in elk ronde 1 minuut de tijd. Het totale aantal goed gemaakte rekensommen van het team wordt dan met de marktprijs vermenigvuldigd, d.w.z. met een factor, die at random door de computer wordt vastgesteld op een waarde tussen 0,75 en 1,25 gulden (in stappen van 5 cent) (1,25 en 1,75 gulden). Deze waarde wordt elke ronde opnieuw vastgesteld, maar wordt pas aan het 
eind van het experiment aan jullie verteld. Van de totale teamopbrengst worden dan 4 gulden afgetrokken en het overblijvende bedrag (de winst) wordt gelijkmatig onder de teamleden verdeeld. Om winst te maken moeten jullie dus samen meer dan 4 gulden per ronde verdienen.

Om een voorbeeld te geven: Als je nu 4 rekensommen goed maakt, en de andere leden van je team ook ieder 4 rekensommen goed maken, dan hebben jullie samen 16 punten. Dit aantal punten wordt met de marktprijs vermenigvuldigd. Als die bijvoorbeeld $\mathrm{f} 0,90$ bedraagt, is de totale teamopbrengst $16 * \mathrm{f} 0,90$ $=\mathrm{f} 14,40$ in deze ronde. Daarvan worden $\mathrm{f} 4,-$ afgetrokken. Dit resulteert in een teamwinst van $\mathrm{f} 10,40$, d.w.z. f 2,60 voor ieder teamlid.

Jullie gaan in totaal 5 ronden spelen, elke ronde met een opnieuw vastgestelde marktprijs.

We beginnen nu eerst met twee rondes, dan komt een andere taak, en dan volgen de laatste drie rondes.

Als je nu nog vragen hebt, geef a.u.b en teken aan de experimentleider!

Je ziet nu straks op het scherm tot welke categorie je teamleden behoren. Als je klaar bent om te beginnen, klik de "ok"-buttonl Als niemand meer vragen heft, begint de eerste ronde.

\section{Instruction 3:}

In het volgende kun je een keuze maken voor een nieuw teamlid. Elk team van "oude" feden krijgt een nieuw fid. Dus, de laatste drie ronden worden met teams van 5 personen gespeeld.

De computer biedt aan elk lid van elk team dezelfde 2 mogelijke nieuwe leden. Elk lid, dus ook jij, moet dan aangeven, of hij/zij een voorkeur heeft voor een van de aangeboden mogelijke nieuwe leden.

Als er geen voorkeur aangegeven wordt, kiest de computer een van de nieuwe leden voor het team met en kans van $50 \%$.

Als je een voorkeur aangegeven hebt wordt je gevraagd om ook nog aan te geven hoeveel je bereid bent te betalen als team om de kans te verhogen dat het door jou geprefereerde nieuwe lid gekozen wordt. Dit bedrag wordt als percentage van de marktprijs van de teamoutput aangegeven. Deze outputprijs is de prijs die geldt voor de ronde die jullie net gespeeld hebben.

Nadat elk lid van elk team zijn beslissing heeft genomen wordt at random de beslissing van een van de teamleden gekozen om uitgevoerd te worden. Als er een voorkeur genoemd en een bedrag aangegeven is, wordt dit bedrag van het team betaald (dus van het tegoed van elk "oude" lid word 1/4 van dit bedrag afgetrokken).

Daarna worden de laatste drie ronden in de nieuwe teams gespeeld en het experiment word met een vragenlijst afgesloten. 


\section{Hieronder zie je en voorbeeld. hoe zo'n beslissing eruit zou kunnen zien!}

Je hebt een voorkeur voor en bepaald nieuw lid aangegeven. Nu moet je beslissen:

Hoeveel ben je bereid te betalen om de kans te verhogen dat het door jou geprefereerde nieuwe lid gekozen wordt? (Het geld wordt door het hele team betaald!)

\begin{tabular}{|l|l|l|l|l|l|l|}
\hline Bedrag in \% van de outputprijs & $0 \%$ & $15 \%$ & $30 \%$ & $45 \%$ & $60 \%$ & $75 \%$ \\
\hline Kans voor het geprefereerde nieuwe lid: & $50 \%$ & $60 \%$ & $70 \%$ & $80 \%$ & $90 \%$ & $100 \%$ \\
\hline
\end{tabular}

Dus, als je b.v. ervoor zou kiezen, $30 \%$ van de outputprijs te betalen, en de outputprijs in de laatste ronde was $f .1 .50$ ( $\mathrm{f} 1.00$ ), dan moet het team dus $\mathrm{f} 0,45$ ( $\mathrm{f} 0,30$ ) gulden betalen voor die voorkeur. Van het tegoed van elk teamlid wordt dan $\%$ van dit bedrag afgetrokken (dus $f 0,11$ ) (dus $f 0.075$ ). Het geprefereerde nieuwe lid wordt nu met een kans van $70 \%$ aan jouw team toegewezen. Ook als het geprefereerde lid toch niet aan jouw team wordt toegewezen, moet je dat bedrag betalen.

Met de nieuwe teams worden dan de laatste drie ronden gespeld!

\section{Instruction 4:}

De laatste drie ronden worden met teams van vijf personen gespeeld. De "nieuwe leden", dus ook jij, worden nu aan de "oude" teams aangeboden, en zij mogen zeggen of ze een voorkeur voor en "blauw" of "rood" nieuw lid hebben.

Uiteindelijk bepaalt de computer wie aan welk "oud" team toegevoegd wordt. Diegenen die aan geen "oud" team toegevoegd worden, spelen dan in een eigen vijf-personenteam verder.

Dus, jullie moeten nu even wachten tot dat de "oude" leden hun beslissingen hebben genomen.

Als de "oude" leden hun beslissingen hebben genomen, horen jullie bij welk team jullie verder gaan spelen.

Met de nieuwe teams worden dan de laatste drie ronden gespeeld.

\section{Questions in the computerized experiments:}

- How old are you?

- What is your sex?

- What do you study?

- Which group has, in you opinion, performed better on the multiplication task?

- How strong did you identify with your category (red/blue)?

- How uncertain did you feel with respect to your results in this experiment?

Resp. exp. 2:

- When you played the game, how well did you understand what happened in this situation?

- When you played the game, how uncertain have you been about what happened in this situation?

- When you played the game, how well could you predict what would happen? 


\section{An empirical test in a business-simulation game ${ }^{65}$}

\subsection{Introduction}

As the EU becomes an ever-closer community, and globalization of firms increases, more and more people work in foreign countries, and people of different nationalities have to work together. Everybody knows about stereotypes of and prejudice against people of other nationalities. Some stereotypes are negative with respect to work behavior, some are positive, and there is reason to assume that they influence behavior. However, despite its relevance for today's world, discrimination by nationality is a topic rarely addressed in economics literature, compared to other kinds of discrimination, e.g., discrimination by race or gender. This chapter explores in how far the economic and psychological mechanisms the integrated framework uses to explain discrimination in general, which have been tested so far using artificial groups, influence discrimination against people of a different nationality.

For the purpose of this study, group discrimination is defined as a preference against having an additional member in one's group only because it belongs to a national outgroup. This holds only if the outgroup can be assumed to be on average at least equally productive as the ingroup. Based on the general framework (chapter 2) and the theoretical model described in chapter 3 , the independent variables assumed to influence the amount of discrimination analyzed here are competition, (self-relevant) subjective uncertainty, and identification with the own group. As described in chapter 3, competition is, following the psychological mechanism, assumed to enhance selfrelevant uncertainty, which leads to strengthened identification with the group one belongs to. Hence, it raises the use of stereotypes and prejudice against the outgroup. The economic mechanism assumed in this chapter differs from the one modeled in chapter 3 : Statistical discrimination is supposed to play a role now, and not only the weak form, as in chapter 4 , but a strong form, as stereotypes about the national groups in question are congruent with real differences in average group performance. Following such an economic mechanism, competition should not allow for discriminatory preferences against the (stereotypically) better performing national group to translate into behavior, when people are interested in the rewards they get from doing well in the game. The

${ }^{65}$ This chapter is based on a working paper jointly authored with U. Glunk (2002) "Nationality-based discrimination in a business-simulation game". Paper presented at the EURAM, Stockholm, 2002. 
theoretical model of chapter 3 implies that the importance of high productivity of new employees in the team's hiring preferences becomes higher when competition strengthens (see the next section). In the business game this study uses (see below), it might even be that participants perceive productivity of a new member as not important at all when competition is low, because there is not much at stake and they might have other aims in the game, such as having fun with their team. However, when they perceive competition to be strong and get involved in this competition, we assume they should also focus stronger on (average) productivities in choosing new members for their teams.

A business simulation game done as part of the regular curriculum for first year 'International Business' (IB) students at the University of Maastricht is used to quasiexperimentally analyze certain aspects of discriminatory behavior between nationalities. The Faculty of Economics and Business Administration at the University of Maastricht is an international faculty, where the working language in most courses is English. The two largest national groups are Dutch and German students. The two groups hold strong stereotypical views about each other, as a pilot survey among 550 first-year students showed (Glunk \& Olie, 2003). The Dutch are portrayed as being more open-minded, but also more arrogant than the Germans, whereas the Germans are said to be more ambitious and hard-working - which is also supposed to lead to better results. Partially, these stereotypes seem to reflect reality, as German students often have better results in their exams, but this does not hold for all courses and in all years, as a comparison of study results of the two groups over several years showed. In more recent years, however, the difference becomes more pronounced than in former periods: In the cohorts of the two management games analyzed here, 2000 and 2001, a larger difference between the two groups than in earlier cohorts exists, German students being better than the Dutch on average. Still, in the 2000-cohort, this effect is only pronounced with respect to methods courses (see table 5.7.4 in appendix I).

The management game provides the opportunity to test some of the predictions of the theoretical model in a more realistic setting than laboratory experiments are. Of course, it is still an artificial situation, but it has many of the features which characterize the real-life situations this research is concerned with: Teams, which work together on a task, have to decide over the composition of the team. The task has some importance for the team as a whole and for each of the team members alone, as students get study points for the management game. The situational context is a simulation of firms on markets, which compete against each other for market share. Last but not least, the categories in 
question are real, meaningful categories, and emotional aspects (prejudice) can influence behavior.

The chapter is structured as follows: First, hypotheses are developed. Secondly, the design of the study is described. Then, the results are presented, and in the last section these results are discussed and a conclusion is formulated.

\subsection{Hypotheses}

The hypotheses are derived from the integrated framework described in chapter 2 , and are related to the integrated model described in chapter 3 . However, they do not mirror the integrated model, as the situation at hand here is a bit different from the one it has been developed for: The integrated model refers to situations where the outgroup is both stereotyped negatively with respect to work performance and disliked (at least because of its outgroup status). In the present case, however, this can hold only for part of the situation, namely for German teams having to decide whether they want a Dutch new member in their group. In the other case, Dutch teams having to decide whether they want a German new member in their group, the outgroup member belongs to a group, which is positively stereotyped with respect to work performance - and these stereotypes are partially in agreement with real average differences between the groups.

Another difference with the integrated model is that in the business game there are no monetary costs: Teams don't pay their members, there is no monetary cost for an implementation of preferences, and teams also don't earn money, but get grades. Finally, there is no individual information, such that use of stereotypes $S=1$ with respect to (valid) performance steretoypes. Therefore, no suppressing economic effect of competition can work on S. The usual cost of imperfect information can be incurred when making the wrong choice for a new team member. In this case, this is not related to monetary profits, but to better or worse grades for team members. The cost of imperfect information becomes a cost of discrimination when preferences are not based on performance-based stereotypes, but on general dislike for an outgroup member, which belongs to a stereotypically better performing nationality. Making a choice based on general dislike for the better performing nationality can lead to lower performance of the own team, and thus reduces the expected grade the team gets for performance in the game. In terms of economic theory, one could speak here about 'taste'-based discrimination as opposed to statistical discrimination. 
The economic mechanism working in this setting is close to the 'strong' version of statistical discrimination: German new members are supposed to be more productive (stereotypically, and for the cohorts at hand this is confirmed by reality), and therefore, 'economically' should be preferred over Dutch new members, when no individual information is available. This economic mechanism and a psychological mechanism based purely on performance stereotypes would lead to the same result - and thus cannot be distinguished. Therefore, this mechanism is referred to as the 'psycho-economic mechanism' in the following. However, a psychological ingroup/outgroup effect (in the following referred to as 'ingroup-bias'), including prejudice, should in this situation lead to a different result, especially as there exists a negative general attitude ${ }^{66}$ towards Germans among many Dutch people (see, e.g., Dekker, 1999), which could strengthen ingroup bias. For German groups deciding about Dutch new members, ingroup bias and productivity-related (stereotypical) perceptions work in the same direction.

In this context it is also important, which stereotypes are focused on - the productivity-related ones, or other stereotypes, e.g., about one nationality being more social than the other. Use of such stereotypes might lead to different evaluations of members of the nationality than productivity-related stereotypes and can influence behavior, especially when students do not see success of their firm as the main aim of participating in the business game. Different from the theoretical model, such stereotypes are introduced here as another determinant of the discriminatory taste $\varepsilon$, besides prejudice.

To link the predictions for the business game with the theoretical model (chapter 3), one can say that two main cases can be distinguished:

1) The less performing national group (Dutch) has outgroup status - $>$ $\left(U^{n \cdot}(\Pi /(P n)) \Delta M P^{67}\right.$ and $\varepsilon^{68}$ reinforce each other and add up to a preference against Dutch new members.

2) The less performing national group (Dutch) has ingroup status $\rightarrow \varepsilon$ works against Germans (the outgroup), $U^{\Pi_{r}}(\Pi /(P n)) \Delta M P$ works in favor of them. $U^{\Pi \cdot}(\Pi /(P n)) \Delta M P$ and $\varepsilon$ counteract each other.

\footnotetext{
${ }^{\infty}$ Not only in the sense of emotional evaluation, but also in the sense of (not-productivity-related) stereotypes.

${ }^{6}$ Now the productivity stereotype refers to nationalities instead of ingroup/outgroup.

The discriminatory taste now is a result not only of prejudice, but also of not-productivity-related stereotypes.
} 
According to the theoretical model, strengthening of competition not only influences the psychological mechanism of ingroup bias ('taste-based discrimination'), but is also expected to affect statistical discrimination. However, as the two mechanisms are assumed to counteract each other in the case when Dutch teams decide about new members, weak competition allows teams to enact the 'psychological' or 'taste-based preference', as productivity-related considerations are seen as being of less importance. Strong competition is expected to reduce the influence of the psychological mechanism in favor of the 'statistical-discrimination effect', because, following the theoretical model, when competition is perceived as strong productivity considerations should play a larger role when choosing a new member. I.e., $U^{\Pi \cdot}(\Pi /(P n))$, which gives the subjective importance of $\triangle M P$ in formula 3.22 for $D U$ with $S=1$, goes up. Following the psychological mechanism, the taste-based preference $\varepsilon$ can also increase with competition. Figure 5.1 illustrates this.

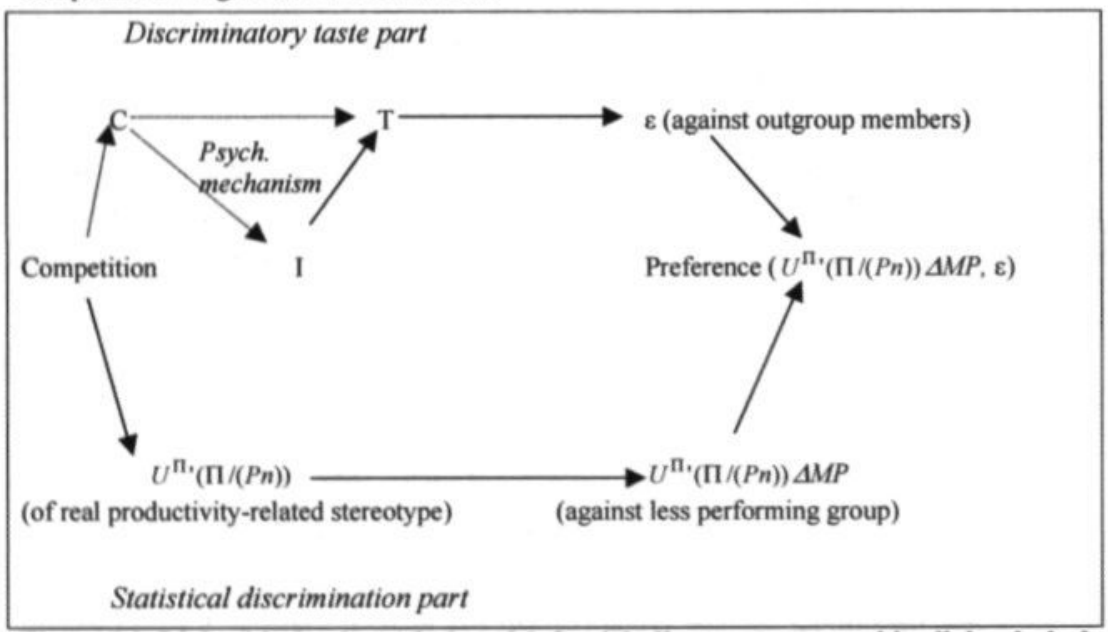

Figure 5.1: Link with the theoretical model. Straight lines represent positive links, dashed lines negative links. $\mathrm{C}=$ self-relevant certainty; $\varepsilon=$ discriminatory taste parameter; $\mathrm{I}=$ identification with ingroup; $U^{\Pi_{1}}(\Pi / P n)=$ marginal utility of real profit share; $\Delta \mathrm{MP}=$ expected difference in productivity.

To summarize the different predictions for the business simulation game: The psychological mechanism predicts that identification with the ingroup (in case of strong/increasing competition) leads to ingroup bias in decision making, which also implies more use of prejudice and (not-productivity-related) stereotypes. Use of 
productivity-related stereotypes can in specific situations also lead to outgroup bias (see chapter 4). Therefore, one should expect a general preference against new members that belong to the national outgroup, but also a preference against members of the stereotypically worse performing nationality. The latter prediction can be based on an economic mechanism: When competition is stronger, and the categories do provide real information about group productivities, members of the group which performs better on average should be preferred, i.e., in this case German new members. 'Economically seen', emotional factors and not-productivity-related stereotypes (general ingroup bias) should not play a role. Therefore, if Dutch students prefer Dutch new members, this indicates a purely psychological mechanism. If Dutch teams prefer Germans, the psychoeconomic mechanism (based on use of productivity-related stereotypes) can explain this. If Germans prefer Germans, it can be the result of both, the psycho-economic or the psychological ingroup mechanism. A situation which cannot be explained, neither with the psycho-economic mechanism, nor with a psychological ingroup effect, would be Germans prefering Dutch new members (outgroup bias in favor of the stereotypically worse performing group). From this reasoning together with the assumption that no significant differences between national groups with respect to general liking of new members exist ${ }^{69}$, the following hypotheses, assuming two main effects and one interaction effect, are derived:

Hla): There is no difference in strength of preferences between teams of different nationalities.

HIb) Germans are preferred over Dutch (main effect of the psycho-economic mechanism; relationship c) in figure 5.2).

$\mathrm{HIc}$ ) Ingroup members are preferred over outgroup members (interaction effect ${ }^{70}$, psychological mechanism; relationship a) in figure 5.2).

Two more hypotheses assume a moderating effect of competition - either for the psycho-economic mechanism or the psychological mechanism of ingroup bias. If the psychological effect of ingroup bias and the psycho-economic effect of stereotype use (statistical discrimination) work in the same direction, both effects cannot be distinguished.

\footnotetext{
${ }^{69}$ Even if the specific Dutch-German situation might lead to stronger ingroup bias among Dutch students, this is not expected to significantly influence general liking for new group members from the national ingroup and outgroup.

7 This referes to the interaction of nationality of the team and nationality of the new member which is expected, and which represents the ingroup/outgroup effect in an ANOVA.
} 
H2a): The stronger competition, the more there is a preference for German new members over Dutch new members (psycho-economic mechanism of stereotype use ('statistical' discrimination; relationships d) - b)-c) in figure 5.2).

$\mathrm{H} 2 \mathrm{~b})$ The stronger competition, the more are ingroup members preferred over outgroup members, no matter what nationality they have (psychological effect; relationships e) - $f$ - a) in figure 5.2).

A precondition for hypothesis $2 \mathrm{~b}$ ) is, that an increase in competition, and hence in self-relevant uncertainty, led to stronger identification with one's ingroup, as the integrated model (chapter 3 ) assumes:

H3): The stronger (perceived) competition, the higher (self-relevant) uncertainty, and hence the stronger identification with one's team/nationality (relationships e) \& $f$ ) in figure 5.2).

The following figure shows the relationships assumed:

\section{Psychological ingroup effect}

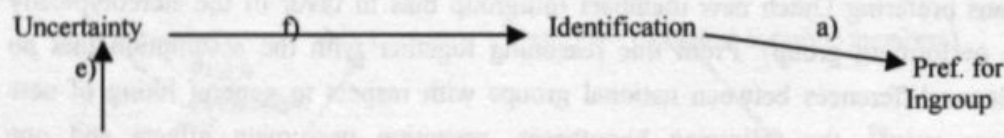

Strong competition

d)

Importance of marginal productivity of new member

Use of (productivity related) stereotypes

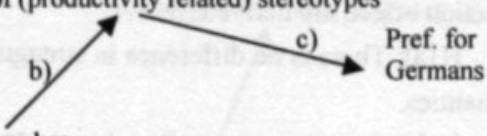

Psycho-Economic effect - statistical discrimination - stereotypes

Figure 5.2: Assumed relationships between variables in the quasi-experiment - straight lines represent positive relationships.

\subsection{Design of the quasi-experiment}

\subsubsection{The management game}

The INTOP III management game is a simulation of firms in a competitive market. More precisely, the game represents a supply oligopoly with polypolistic demand (see Baetge, Fischer, \& Kunberger (1998) for a more detailed description of the game). Two products are produced (vacuum cleaners and radios) at different quality levels. Teams of maximally six players represent one firm, which is competing with four other 
firms in one game. Players have to make decisions concerning the amount of production, diversification of products, expansion to a foreign market, marketing strategies etc., i.e., strategic decisions concerning business objectives and operating principles (Baetge, Fischer, \& Kunberger, 1998). Each player is supposed to be mainly responsible for one area of decisions. The game is played over six rounds. It is played computer-based, and students get help from tutors when they have problems with the tools, but they have to make their business-related decisions without help. A computer calculates market processes. Human resource management or other labor-related questions are not touched. However, the game requires team skills of the participants. Competition usually is perceived as strongest in the third round, as then all difficulties are introduced and all participants usually have understood the game correctly. However, if a firm has done very badly in round one, it is difficult to catch up again later.

This game provides a unique environment for a quasi-experimental study. The setting simulates a business environment, participants have to make business decisions, and they have to do this in interdependent teams. Furthermore, there is something at stake in this game, not monetary profits, but grades for the course. Still, the setting is relatively controlled such that it is close to a laboratory experiment in a more complex setting. However, as the game is played for certain educational purposes, it is not possible to control for all factors one would like to control for. For example, how teams structure their decision-making process is up to them, and free communication is allowed. As several teams play in the same room, inter-team communication is in principle possible (but not very probable).

\subsubsection{Sample}

The sample of the first game consisted of 57 teams. Four teams including nonDutch, non-German students, and two teams with equal numbers of Dutch and German students were excluded from the analysis, as there were too few of them for a separate analysis. Of the 51 teams left, 35 were homogeneous (25 Dutch, 10 German) and 16 included a token person or a minority (six with German majority, ten with Dutch majority).

With respect to the discrimination measure (see below for details), in total 29 groups answered the question whether a new member may join their group about a German student, and 22 groups about a Dutch student. For ten groups the new member was an ingroup member (two German new members, eight Dutch new members), for 14 
German and 27 Dutch groups it was an outgroup member. All members of each team answered the questionnaires, so altogether, 217 students where taking part in the study. Team size varied between four and six people.

The sample of the second game consisted of 55 teams taking part in the management game. For the analysis eight teams with non-Dutch and non-German members, two half-Dutch, half-German teams, and one team with members of unknown nationality were excluded. Of the remaining 44 teams, 22 were homogeneous - 11 Dutch and 11 German - and 22 included a token person or a minority. Five groups had a German majority, and 17 groups had a Dutch majority.

With respect to the discrimination measure, 42 of the teams included in the analysis answered the question about either a Dutch or a German student. 17 answered this question about a German student, and 25 groups about a Dutch student. 15 Dutch majority groups answered the question for an ingroup member, and 12 for an outgroup member, while five German majority groups answered the question for an ingroup member and 10 for an outgroup member. The number of members of one team answering the questionnaire varied with team-size between three and seven people ${ }^{71}$. In general, nearly all members of each team answered the questionnaires, so altogether, 297 students were taking part in the study.

\subsubsection{Procedure and questionnaire}

Data were collected during two consecutive years. The reasons for running the study twice were (i) that one management game does not provide enough aggregate data for analyzing questions of ingroup-outgroup favoritism by nationality with reasonable sample size, and (ii) that preliminary results from the first experiment raised some questions, which could not be answered with the data collected. Therefore, some new items were included in the second questionnaire. Data in general was pooled for the two years, as one can assume that the sample of both years comes from approximately the same population, business students at the University of Maastricht. A test whether samples from the two years differed in the discrimination measure (which measured liking of a specific new member - see below) was negative $(t=.38, p=.707$ (2-tailed); see table 5.7 .3 in appendix I).

Hypotheses 1 and 2 were analyzed with pooled data, whereas hypothesis 3 was analyzed for the two games separately. This was done because for measuring

\footnotetext{
$"$ One team consisting of only two people was excluded from the analysis.
} 
identification and uncertainty different questions were used in the second study. The reason for changing parts of the questionnaire was that in the first game it had been very long, and this led to a relatively high amount of incompletely answered questionnaires. The scales for measuring identification and certainty therefore consisted of fewer items in the second game.

In both games, the same questionnaire was given to the students at three points in time during the game. However, for the research reported here only the second measurement time is relevant. The questionnaire consists of items concerning perception of the team situation, stemming mainly from widely used and tested instruments. In the second game, apart from shortening some scales, some items were added, but the main part remained the same. Relevant here are the following parts of the questionnaire:

In the first game, thirteen items adapted from Mullin and Hogg (1998) were used to measure identification with the team ("I have positive feelings towards my team members; I have confidence in my team members; I feel comfortable depending on my team members; I think the other team members perform well; I think that, with regard to decision making, 1 have more in common with members of this team than with members of other teams; I think that, generally speaking, I have more in common with members of this team than with members of other teams; I think that the members of this team are well-suited to each other; I think it is right, in the view of my individual performance, that I am a member of this team; I find it pleasant to be a member of this team; I would like to continue working together with this team; I think it would be nicer in another team; I am glad that I ended up in this team; I have negative feelings about my team members"). Cronbach's alpha ${ }^{12}$ of this scale is .83. In the second game, only six items remained in the scale ("I have positive feelings towards my team members; I have confidence in my team members; I feel comfortable depending on my team members; I think the other team members perform well; I think that the members of this team are well suited to each other, I would like to continue working together with this team"). Cronbach's alpha of this scale was .96 .

In 2000, five self-constructed questions asked how certain subjects were about various aspects of their firm's performance and strategy in the game, representing the construct 'self-relevant (un)certainty' ("How certain are you that you are personally

\footnotetext{
${ }^{n}$ Cronbach's alpha is a coefficient of reliability and measures how well a set of items (or variables) measures an one-dimensional latent construct. It is a function of the number of test items and the average intercorrelation among the items (see https//www. ats. ucla.edu/stat/spss/faq/alpha. html).
} 
doing well in this game?"; "How certain are you that your firm will be doing well in this game?"; How certain are you about the measures your firm has to take to do well in this game?"; How certain are you that you will succeed with your strategy?"; How certain are you that you will reach your personal aims during this game?"). These questions were constructed such that they covered different aspects of uncertainty - about results in the game, but also uncertainty about doing personally well and about being able to reach personal aims in the game, as aims of participants could differ from doing well as a team in the game.

In the second game only three, reformulated, questions measuring uncertainty were asked ("When playing the Management Game, ...to what extent do you understand it?"; "... are you certain about what is happening in the game?" " “... can you predict what is going to happen in the Management Game?"). Cronbach's alpha of the uncertainty scale was .92 in the first game and .76 in the second game. The reason for changing the uncertainty measure from the first game despite of a very high alpha was the intention to shorten the questionnaire. Furthermore, tutors indicated that one important source of uncertainty could be understanding of what happened in the game. This is a more basic uncertainty than the one captured with the items in the first game, so that it was assumed that questions pertaining to this aspect could capture student's subjective uncertainty equally well, in a shorter way. This, however, was not confirmed with respect to internal consistency of the scale. The aim was of course to represent the construct "self-relevant subjective certainty" as well as possible, but already the experiments described in the previous chapter showed that it is difficult to measure this construct validly. Further research should try to develop a more valid scale.

Only in the second game, one self-constructed item measured whether the efforts of the team were seen as related to the actual team results ("When playing the Management Game, to what extent do you see the efforts of your team as related to the actual team results?"). We decided to measure this, because it would make a difference especially with respect to the 'economic' mechanisms, whether students attribute the success in the game rather to chance or to the effort of the team.

In the first game, one self-constructed item measured how important it is for the participants to do well in the game ("How important is it for you to do well in this game?"). In the second game, seven self-constructed items measured what aspects of the game were important for the participants: "How important is it for you, ... to do well as a firm in the game?; ...that your team outperforms the other teams?; ...that everybody of 
your team contributes equally to the success of the firm?; ... to have fun during team meetings; ...to socialize during team meetings?; ...to get along well with your team members?"). This scale was extended from one question to these six questions, to be better able to rule out an alternative explanation for the findings in game one, namely that Dutch subjects see performing well as less important than German subjects, and maybe have rather 'social' aims.

Perceived strength of competition in both games was measured with one item asking team members how strong they perceive competition between the firms in their game to be ("How strong is in your perception the competition between the firms?").

To measure discrimination, in both years teams were asked, ostensibly by the block-coordinator, whether they would like to have one additional member in the group. The potential additional member was described as an one-year older student who wants to tutor the management game in the year to come, having studied at a different university before and therefore being without own experience of this management game. Either he had a German name and supposedly came from the university of Cologne, or a Dutch name, supposedly coming from Rotterdam ${ }^{n}$. Each member of the team was asked to privately answer the question in how far he/she would like to have the new member in the team (see appendix II for the wording of this question). The measure was constructed such that the two new members were as similar as possible, varying only in name and university ${ }^{74}$ visited before. The story had to be convincing, and that is why it was chosen for someone from the second study year, as older students often tutor in the management game. Furthermore, being older, and therefore having more general economic knowledge, should make the student in general attractive as a new member for the team.

Most items were measured on five-point Likert scales, only the discrimination measure ('strength of wanting a new member') was measured on a continuous scale ranging from zero to ten, to allow for more precise expressions of preference. The response rate of the questionnaire at time two (round three) was $75 \%$, equally in game one and game two.

\footnotetext{
${ }^{73}$ This constitutes deception, but it was necessary to have a believable coverstory for the discrimination measure, as otherwise, social-desirability considerations would have influenced the data even more strongly.

${ }^{74}$ There is a chance that the image of the university the student comes from influences perceptions as well, but at least (informal) pretests among students did not show any differences in evaluation of the two universities.
} 
As the discrimination measure was collected as a between-subjects (groups) factor, i.e., each group was either asked about a Dutch or about a German new member, it is in fact not possible to say whether specific teams 'preferred' an ingroup over an outgroup member. The question answered is rather whether ingroup members or a certain national group offered to a team were liked more than outgroup members or the other national group. If we still speak about 'preference', it has to be kept in mind, that teams did not get an 'either-or' choice, but were asked about one new member only. This was done to avoid problems with social desirability concerns, which would have come up when students were given the choice between two new members from different nationalities. Furthermore, giving them two equal descriptions of a person only differing in nationality, would not have been very credible for students, and they would have guessed that the choice they make is not real. We borrowed from the design of the audit studies reviewed in section 2.2 .

\subsection{Results pooled data}

Data are aggregated on group level for the analysis. Separate analyses are done for data of homogeneous groups (HOM) and for data including heterogeneous groups (with a majority of one nationality; HET). Hypotheses one and two were analyzed using pooled data, as for these analyses only variables are used which were measured in exactly the same way in the two years. For the analysis of hypothesis three and some exploratory analyses, data from the two games were separated. In the following, all analyses are described first for the homogeneous data alone and then for the data of homogeneous and heterogeneous groups pooled. In general, t-test statistics were calculated, as sample size for most tests was reasonably large and the distribution of the data did not differ significantly from a normal distribution (see tables 5.7.1 and 5.7.2 in appendix I). Again, also marginally significant results ( $10 \%$ level) are reported, but these must be interpreted carefully (see chapter 4 ). Sample sizes might occasionally vary slightly between the different analyses, as there are missing data for all variables, and the amount of missing data differs between variables, because questionnaires sometimes were filled in incompletely.

\subsubsection{Hypothesis (a) - main effect of nationality}

German teams are significantly more willing to accept new members than Dutch teams are, and this holds for homogeneous groups separately as well as for all groups 
together (HOM: $\mathrm{t}=-3.41, \mathrm{p}=.001 ;$ HET: $\mathrm{t}=-2.83, \mathrm{p}=.006$ (2-tailed)). Hypothesis la) thus had to be rejected.

\begin{tabular}{cccccc}
\hline Data & $\begin{array}{c}\text { Nationality of } \\
\text { group }\end{array}$ & N Mean & Std. Dev. & $\begin{array}{c}\text { Std. Err. } \\
\text { Mean }\end{array}$ \\
\hline HOM & Dutch & 34 & 3.98 & 3.01 & .52 \\
& German & 20 & 6.91 & 3.12 & .70 \\
\hline HET & Dutch & 63 & 4.42 & 3.23 & .41 \\
& German & 33 & 6.33 & 3.01 & .52 \\
\hline
\end{tabular}

Table 5.1: Descriptive measures for liking of new members by nationality of group

\subsubsection{Hypothesis Ib) (main effect psycho-economic mechanism)}

There is no general difference in being liked between Dutch and German possible new members in general. Including the heterogeneous teams, there is a marginally significant difference between the two nationalities in being liked as new members contrary to the predictions, the Dutch are liked slightly better (HET: $t=1.89, p=.060$ (2tailed))

\subsubsection{Hypothesis Ic) (interaction effect psychological mechanism)}

Table 5.2 shows that there is a tendency that ingroup members are preferred over outgroup members as new members for the team in homogeneous teams. However, this effect is only marginally significant $(t=1.31$; sig. $=.098$ (1-tailed)). If one adds the heterogeneous teams the picture changes: Ingroup members are significantly preferred over outgroup members now $\left(t=1.19 ; p=.027(1 \text {-tailed })^{75}\right)$. Thus, hypothesis $\left.1 \mathrm{c}\right)$ is confirmed by the data for heterogeneous groups.

\begin{tabular}{cccccc}
\hline Data & New member is N & Mean & Std. Dev. & Std. Err. Mean \\
\hline HOM & ingroup & 17 & 5.94 & 3.75 & .91 \\
& outgroup & 37 & 4.67 & 3.10 & .51 \\
& Dutch & 25 & 5.60 & 3.70 & .74 \\
& German & 29 & 4.61 & 2.98 & .55 \\
HET & ingroup & 30 & 5.99 & 3.45 & .63 \\
& outgroup & 65 & 4.61 & 3.12 & .39 \\
& Dutch & 48 & 5.66 & 3.37 & .49 \\
& German & 47 & 4.41 & 3.07 & .45
\end{tabular}

Table 5.2: Descriptive measures for liking of new member with respect to type of new member. Measured on a scale from $0-10$ with 5 being indifference.

${ }^{75}$ Ingroup/outgroup always with respect to the majority in the teams. 
An analysis of variance including both factors, nationality of team and nationality of the new member, and an interaction term representing ingroup-outgroup status was additionally conducted to test which effect is strongest.

For homogeneous data alone, the ANOVA shows that only the main effect for nationality of the team becomes significant, i.e., nationality of the team alone explains preferences for new members. If heterogeneous data are included, the interaction effect, i.e., the ingroup/outgroup effect, becomes significant, too. This is in part surprising, as heterogeneous teams already include outgroup members and one could think that they should rather tend to less ingroup bias (see also figures 5.7.1 and 5.7.2 in appendix I for a graphical representation of the interaction effect).

\begin{tabular}{cccccc}
\hline Data & $\begin{array}{c}\text { Nationality of new } \\
\text { member }\end{array}$ & Nationality of team Mean & Std. Dev. & N \\
\hline HOM & Dutch & Dutch & 4.67 & 3.99 & 11 \\
& German & German & 6.33 & 3.43 & 14 \\
& Total & 5.60 & 3.70 & 25 \\
& Dutch & 3.65 & 2.45 & 23 \\
& Total & German & 8.27 & 1.77 & 6 \\
& & Total & 4.61 & 2.98 & 29 \\
& Dutch & 3.98 & 3.01 & 34 \\
& Dutch & German & 6.91 & 3.12 & 20 \\
& & Total & 5.07 & 3.34 & 54 \\
\hline HET & Dutch & 5.45 & 3.64 & 23 \\
& German & German & 5.86 & 3.17 & 25 \\
& & Total & 5.66 & 3.37 & 48 \\
& Dutch & 3.82 & 2.84 & 40 \\
& & German & 7.80 & 1.92 & 8 \\
& Total & Total & 4.49 & 3.08 & 48 \\
& & Dutch & 4.42 & 3.23 & 63 \\
& & German & 6.33 & 3.01 & 33 \\
& Total & 5.07 & 3.27 & 96 \\
\hline
\end{tabular}

Table 5.3: Descriptive statistics, dependent variable: Liking of new member. 


\begin{tabular}{rrrrrrr}
\hline Data & Source & Sum of Squares & df & Mean Square & \multicolumn{1}{c}{ F } & Sig \\
\hline HOM & Corrected Model & 131.63 & 3 & 43.88 & 4.78 & .005 \\
& Intercept & 1411.05 & 1 & 1411.05 & 153.59 .000 \\
& Nationality of new member & 2.26 & 1 & 2.26 & .25 & .622 \\
& Nationality of team & 105.73 & 1 & 105.73 & 11.51 .001 \\
& Ingroup/outgroup & 23.48 & 1 & 23.48 & 2.56 .116 \\
Error & 459.35 & 50 & 9.19 & \\
Total & 1977.88 & 54 & & \\
\hline HET & Corrected Total & 590.98 & 53 & & \\
& Corrected Model & 140.67 & 3 & 46.89 & 4.94 .003 \\
Intercept & 2251.71 & 1 & 2251.71 & 236.99 .000 \\
& Nationality of new member & .42 & 1 & .42 & .04 .834 \\
& Nationality of team & 82.54 & 1 & 82.54 & 8.69 .004 \\
Ingroup/outgroup & 54.31 & 1 & 54.31 & 5.72 .019 \\
Error & 874.11 & 92 & 9.50 & \\
Total & 3486.28 & 96 & & \\
\hline
\end{tabular}

Table 5.4: Univariate analysis of variance for the dependent variable 'preference for a new member'; HOM: $\mathbf{R}^{2}=.22$ (adjusted $\mathrm{R}^{2}=.18$ ); HET: $\mathbf{R}^{2}=.14$ (adjusted $\mathrm{R}^{2}=.11$ ); Type III sum of squares.

\subsubsection{Hypothesis 2a) ('statistical' discrimination)}

Looking at preferences by nationality of the new member for homogeneous teams, there is no correlation between competition and liking of a new member (for Dutch new members $r=-.16, p=.58$, for German new members $r=-.28, p=.24(2$ tailed)).

If heterogeneous data are included, the negative correlation of strength of competition with liking of German new members becomes significant at the $10 \%$ level $(r$ $=-.28, p=.07$ (2-tailed)), which is contrary to our hypothesis. Germans are the less liked as new members the stronger competition is, whereas no such link for Dutch new members exists $(r=-.15, p=.61)$.

\subsubsection{Hypothesis 2 b)(psychological effect of competition)}

For homogeneous data, strengthening competition has no significant effect on liking of an ingroup member, but it reduces the amount of liking of an outgroup member (marginally significant; ingroup member $\mathrm{r}=-.38, \mathrm{p}=.14$; outgroup member $\mathrm{r}=-.30, \mathrm{p}=$ .08 (2-tailed)). This partially confirms hypothesis $2 \mathrm{~b}$ ).

If the heterogeneous data are included, none of the correlations becomes significant anymore (ingroup $\mathrm{r}=-.21, \mathrm{p}=.28$; outgroup $\mathrm{r}=-.19, \mathrm{p}=.13$; (both 2-tailed)). 


\subsubsection{Exploratory analysis}

After a general effect of the nationality of the team on preferences for new members had been found, we also tested whether nationality of the team determines whether competition influences liking of new members. For homogeneous data alone there is indeed a difference between German and Dutch teams, in that the negative correlation between competition and liking of a new member only (marginally significantly) holds for German teams (Dutch teams:

$r=-.24, p=.176$; German teams $r=-.40, p=.087$ ). Including heterogeneous teams, no significant correlation for any of the groups is found.

\subsection{Results game 1 and game 2 separately}

In the following, the analysis is again done first for homogeneous groups only, and in a second step for homogeneous and heterogeneous groups (with a majority of one national group) together.

\subsubsection{Hypothesis 3 (background conditions)}

Game 1: Hypothesis 3 is for homogeneous teams confirmed by the data: Stronger perceived competition correlates significantly positive with identification with one's group $(\mathrm{r}=.34, \mathrm{p}=, 026$ (1-tailed)). Including heterogeneous teams, no significant correlation between perceived strength of competition and identification remains.

It is not so clear what direction for a correlation of uncertainty with identification to expect at this point of the game. Theoretically, the less certain someone is, the stronger should he identify, which would imply a negative relationship. But, when players filled in the questionnaires, they already had played some rounds in their teams, so it could well be that students who were uncertain in the beginning identified strongly with their team and therefore now were more certain, i.e., a positive correlation between identity and certainty could be expected (see also section 4.3). Therefore, a two-tailed test of significance was done. Certainty and identity correlates significantly positive with each other, suggesting that students who identified stronger with their teams got some 'certainty' from this (HOM: $\mathrm{r}=.47, \mathrm{p}=.006$ ). This held for homogeneous and heterogeneous teams equally (HET: $\mathrm{r}=.45, \mathrm{p}=.002$ ).

Game 2: There is a significant correlation of competition with identification for homogeneous data $(r=.41, p=.036(1$-tailed)). For heterogeneous data, this correlation is only marginally significant $(r=.18, p=.096)$. Furthermore, the stronger subjects 
identify with their group, the stronger is also their certainty $(\mathrm{HOM}: \mathrm{r}=.52, \mathrm{p}=.000$; HET: $r=.31, p=.024$ (2-tailed)). Hypothesis 3 is thus confirmed in both games, especially for homogeneous groups.

\subsubsection{Exploratory analyses for the two games separately}

Relationship between identification and liking of a new member: For homogeneous and heterogeneous teams, in game $I$, identification correlated negatively with the willingness to get a new member for the team, i.e., the stronger a team identifies, the less it wants a new member (HOM: $\mathrm{r}=-.37, \mathrm{p}=.036$; HET: $\mathrm{r}=-.34, \mathrm{p}=.02$ (both 2-tailed)).

Split up by ingroup-outgroup status of the new member, it becomes visible that this is only (marginally) significant for outgroup members (HOM: $r=-.36, p=.433$ for ingroup; $\mathrm{r}=-.37, \mathrm{p}=.064$ for outgroup; HET: $\mathrm{r}=-.414, \mathrm{p}=.268$ for ingroup; $\mathrm{r}=-.30, \mathrm{p}$ $=.073$ for outgroup (2-tailed)).

In game 2, looking at homogeneous data only, there is no significant correlation between identification and general liking of a new member $(r=-.20 ; p=.42$ (2-tailed)). Including heterogeneous data, the correlation remains insignificant $(r=-.22, p=.13)$. Correlations between identification and preferences for ingroup res. outgroup members do neither become significant for homogeneous nor for heterogeneous data.

Relationship of importance of good performance with liking of a new member: In a further exploratory analysis it was tested, whether there is a difference between Dutch and German subjects in the variable 'importance to do well in the game'. This was done because the stereotypes about the two groups as described in section 5.1 also suggest that there might be such a difference, and this could influence preferences.

Neither in game 1 nor in game 2 a significant difference was found between Dutch and German (majority) groups in any of the variables measuring 'importance to do well' (game 1) or 'aims' students have (game 2).

Relationship of relevance of team effort with liking of a new member:Another explanation for economically not 'rational' preferences with respect to the performance of new members, i.e., preference for the group performing worse, could be that participants do not think that team effort is relevant for the results in the game. The variable measuring 'relevance of team effort' was measured only in game 2 . It correlates negatively with liking of any new member. This correlation is marginally significant for homogeneous groups and becomes significant at the $5 \%$-level when heterogeneous teams are included in the analysis (HOM: $\mathrm{r}=-.41, \mathrm{p}=.079 ;$ HET: $\mathrm{r}=-.35, \mathrm{p}=.013$ (2-tailed)). 
Looking at liking of a specific member, for homogeneous groups the correlation is (marginally) significant for preference against a German new member, but not significant for preference against a Dutch new member (Dutch $\mathrm{r}=-.27, \mathrm{p}=.429$, German $\mathrm{r}=-.65$, $\mathrm{p}$ $=.084$ (2-tailed)). I.e., the more important team effort is perceived to be, the less German new members are liked for the team. On the other hand, if heterogeneous data are included, the correlation becomes significant at the $5 \%$ level for Dutch new members and not significant for German new members (Dutch $\mathrm{r}=-.38, \mathrm{p}=.044$; German $\mathrm{r}=-.30, \mathrm{p}=$ .182). Heterogeneous teams having experienced both nationalities obviously do not like having Dutch new members when team effort is seen as important.

If data is split by whether the new member is from the ingroup or outgroup, no significant relationship between perceived relevance of team effort and liking of any kind of new member for homogeneous data was found. Including heterogeneous data, the correlation becomes only significant for preference against outgroup members (ingroup $r$ $=-.22, \mathrm{p}=.369$; outgroup $\mathrm{r}=-.46, \mathrm{p}=.043$ ), i.e., the more important team effort is experienced to be, the more the majority nationality is preferred. Figure 5.3 shows the relationships found.

\section{Psychological ingroup-effect}

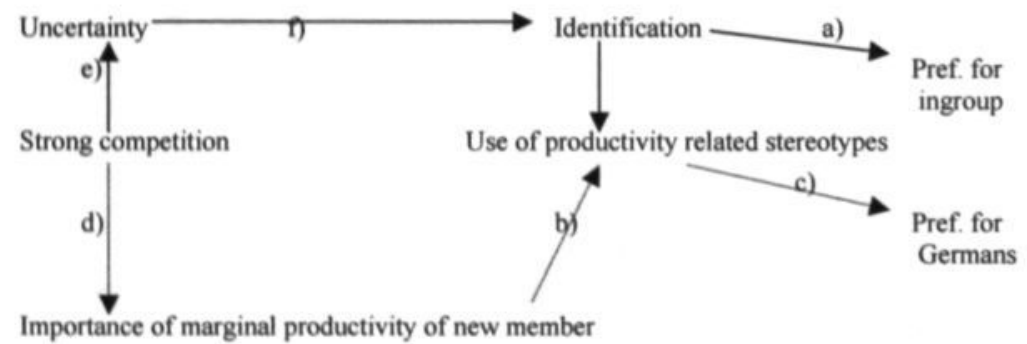

Psycho-Economic effect - statistical discrimination - stereotypes

Figure 5.3: Relationships between main variables - dashed lines represent hypotheses, which were not confirmed, full lines confirmed relationships ${ }^{76}$. All relationships had been assumed to be positive (see figure 5.2).

\footnotetext{
\% The figure does not represent all results, as the fact that nationality of the team played a main role in determining behavior was not represented in the first graph as an hypothesis either.
} 


\subsection{Discussion and conclusion}

The results confirm the idea that psychological effects play an important role in determining behavior in an 'economic' setting.

First, however, an effect was found which was not expected and cannot easily be explained: There is a significant difference between the two nationalities in how far they were willing to accept new members. German teams are significantly more willing to accept new members than Dutch teams are.

Secondly, ingroup bias was found and was stronger when heterogeneous teams were included in the analysis. This is surprising, as one might expect heterogeneous teams to get accustomed to the minority and therefore show less ingroup bias. However, it seems rather to be the case that heterogeneous teams try to avoid that the minority gets 'stronger'. It might even be that minority members in these teams act the same way as majority members. Unfortunately, this cannot be analyzed with the data at hand, because they do not allow for differentiating whether an answer to the 'discrimination measure', i.e., the question measuring liking of a new member, stems from a majority or minority member in a heterogeneous group.

There was no specific bias against one national group in general, and in the ANOVA the main effect for 'nationality of new member' did not become significant, neither with nor without heterogeneous teams.

The finding that in general German students were not preferred as new members, which is contrary to what the psycho-economic meachnism of performance stereotyping predicted, could have several reasons, apart from indicating that psychological forces of ingroup bias can be stronger than more 'economic' forces: The relations between Germans and Dutch are difficult, as the memory of the Second World War is still kept strong in the Netherlands (see e.g., Dekker, 1999). For the same reason, Dutch students would admit faster that they don't like a German member in their group. German students would probably rather not admit that they don't want a Dutch student in their group.

Apart from special German-Dutch factors, another explanation for the finding that in general German students are not preferred as new members could be that, as already Roethlisberger \& Dickson (1939) showed, overachievers are not liked in teams, because they enhance the standards to be fulfilled. Still another possible explanation, inferred from the stereotypes of the two groups, could be that German and Dutch students have different aims in playing the game, i.e., it might be more important for 
German students to perform well, and more important for Dutch students to have a good time with the team. But, German and Dutch teams were not found to differ in how important they think it is to do well in the game, such that this explanation does not hold.

However, German students showed stronger ingroup bias than Dutch students, albeit being rather positive about both possible new members. That can be interpreted as evidence for an interaction of the psycho-economic and psychological mechanisms, strengthening ingroup bias when the ingroup is the better performing group, and weakening ingroup bias when the ingroup is the worse performing group.

A possible explanation for the finding that Dutch teams in general are less prone to accept new members than German teams could be that Dutch students did not perceive a Dutch student coming from Rotterdam as an ingroup member. For them, living in their own country, nationality is a less salient category than region, while for German students studying abroad nationality is a very salient category, so that the student from Cologne was probably perceived as belonging to the German ingroup.

Strengthening of competition has been found to influence the liking of new members for the team, but in a different way than the theoretical model predicts. In general, strengthening competition reduces liking of any kind of new member. More specifically, stronger competition is not associated with stronger liking of new members from the stereotypically better performing group, but with preference against outgroup members for homogeneous teams, and with preference against German new members when heterogeneous teams are included. This means, especially homogeneous teams rather 'close ranks' when competition gets stronger and do not want an outgroup member in the team, even if the outgroup is stereotyped in a way that makes them appear more productive. An explanation for the fact that the link between competition and discriminatory tendency gets weaker when heterogeneous teams are included in the analysis could be that these teams do not react so strongly to a change in competition, but rather show ingroup bias in all competitive situations. Again, unfortunately, this cannot be analyzed for the different national groups within a team separately.

The psychological effect that stronger competition should lead to stronger identification was confirmed for homogeneous teams in both games, and in game 2 marginally significantly also for the data including heterogeneous teams. Members in heterogeneous teams might 'assume' (not necessarily consciously) not to get that much certainty from identifying stronger with their teams - maybe just because teams are 
heterogeneous $"$. However, in general, the stronger subjects identify with their teams, the more certain they are, which together with the aforementioned hypothesis confirmed hypothesis 3 for homogeneous teams and partially also for heterogeneous teams.

A further interesting finding is that in game 1 teams who take the game more seriously are less willing to accept a new member for the team, and this holds for homogeneous and heterogeneous teams, and to a higher extent against outgroup members. Majority groups seem to try to avoid more strongly that the teams get (additional) outgroup members when they are more inclined to do well in the game.

The finding that teams which believed more strongly that their joint effort is relevant for the outcome did not want new members, and no German new members as well, also does not fit with an economic explanation, which would predict a preference for Germans, as they are the higher performers. A possible explanation is that team performance is not only determined by the sum of individual performances, but also by how well the team cooperates. This might in general become more difficult in heterogeneous teams, and therefore, such preferences can be 'rational' in cases were team performance is seen as very important. Heterogeneous teams were able to base their choice on experiences made with the minority in their teams.

From comments we got from our participants we saw that a further, more tangible factor influencing preferences is language. On some questionnaires people had written sentences like "it's okay if he enters, but he will have to speak Dutch". If one wants to have nationally heterogeneous teams, it is obviously important that the language problem is solved in a way that everybody really feels comfortable with the common language used.

The study provides some evidence for the importance of psychological mechanisms in economic settings. Not only expected productivity influences 'hiring decisions' in a business game, even if performance is important.

The analysis of the economic effects of competition in this study however faces some problems. The game is not very important for the grade of the students, because individual grades formed the larger part of the final grade of students in this course. Therefore, 'economic' considerations do not play such a big role, while 'psychological' considerations can influence behavior more easily.

\footnotetext{
$\pi$ There is however no general tendency of heterogeneous groups to identify less strongly than homogeneous teams (game $1: t=-72, p=.477$, game $2: t=1.64, p=.109$ )
} 
A further problem was the trade-off in measuring discrimination between the reduction of the tendency to answer the question in a socially desirable way and the aim of getting as much informative data as possible. A choice was made in favor of an anonymous assessment of the discrimination measure, which did not allow for testing whether minorities and majorities in heterogeneous teams decide differently and are differently influenced by the factors analyzed.

The results of this study are only partially generalizable to real-life settings for several reasons. First, in this game performance was not that important, as said before. Secondly, grades and money are different kinds of incentives and may influence behavior in a different way. Third, it was 'just' a game, and participants were not employers, but students with limited knowledge and acting in a very stylized business environment.

Still, the study provides some indication from a more complex and realistic setting as compared to a laboratory experiment that the mechanisms proposed by the integrated framework play a role in determining preferences for new team members. Psychological mechanisms have been slightly stronger than economic mechanisms in this study. Further research should try to extend the analysis to field settings or find controlled settings were incentives are closer to the ones found in economic reality. Furthermore, it became obvious that 'real-world' stereotypes are quite complex and can influence behavior in many ways. Further research therefore should analyse these aspects more closely, to be perhaps also able to find ways to reduce negative discrimination by focusing on 'positive' aspects of the stereotypes. But, it should always be kept in mind that these stereotypes and preferences continue to exist in the heads of the people involved and might influence cooperation in heterogeneous teams negatively, even if there is no actual discrimination in hiring. 


\section{Appendix I: Tables and figures}

\begin{tabular}{cccccc}
\hline & $\begin{array}{c}\text { Discrimination } \\
\text { measure }\end{array}$ & $\begin{array}{c}\text { Importance to } \\
\text { do well }\end{array}$ & $\begin{array}{c}\text { Perceived } \\
\text { competition }\end{array}$ & $\begin{array}{c}\text { Certainty } \\
\text { Identification } \\
\text { with ingroup }\end{array}$ \\
\hline $\mathrm{N}$ & 51 & 47 & 47 & 47 & 46 \\
Normal Pars. Mean & 5.15 & 3.70 & 3.66 & 3.45 & 3.65 \\
$\quad \begin{array}{c}\text { Std. Dev. } \\
\text { Most Extreme Absolute }\end{array}$ & 3.17 & .52 & .49 & .48 & .41 \\
$\begin{array}{c}\text { Differences } \\
\text { Kolmogorov- }\end{array}$ & .09 & .13 & .16 & .08 & .09 \\
$\begin{array}{l}\text { Smirnov Z } \\
\text { Asymp. Sig. }\end{array}$ & .66 & .90 & 1.08 & .56 & .64 \\
(2-tailed) & .776 & .39 & .19 & .91 & .81 \\
\hline
\end{tabular}

Table 5.7.1: One-Sample Kolmogorov-Smirnov Test for normal distribution: game 1.

\begin{tabular}{|c|c|c|c|c|c|c|c|}
\hline & & $\begin{array}{c}\text { Discriminatio } \\
\text { measure }\end{array}$ & $\begin{array}{l}\text { n Perceived } \\
\text { competition }\end{array}$ & Certainty & $\begin{array}{l}\text { ydentificatic } \\
\text { with ingrou }\end{array}$ & $\begin{array}{l}\text { onlmportance } \\
\text { ip to have fun }\end{array}$ & $\begin{array}{l}\text { Import to } \\
\text { perform } \\
\text { well }\end{array}$ \\
\hline $\mathrm{N}$ & & 40 & 43 & 43 & 43 & 43 & 43 \\
\hline Normal Pars. & Mean & 4.96 & 3.44 & 3.35 & 4.03 & 4.15 & -.46 \\
\hline & $\begin{array}{l}\text { Std. } \\
\text { Dev. }\end{array}$ & 3.50 & .51 & .37 & .50 & .43 & .53 \\
\hline $\begin{array}{c}\text { Most } \\
\text { Extreme } \\
\text { Differences }\end{array}$ & Absolute & .15 & .13 & .11 & .07 & .11 & .15 \\
\hline $\begin{array}{l}\text { Kolmogorov- } \\
\text { Smimov Z }\end{array}$ & & .92 & .86 & .75 & .48 & .71 & .96 \\
\hline $\begin{array}{l}\text { Asymp. Sig. } \\
\text { (2-tailed) }\end{array}$ & & .37 & .45 & .63 & .97 & .69 & .32 \\
\hline
\end{tabular}

Table 5.7.2: One-Sample Kolmogorov-Smirnov Test for normal distribution: game 2.

\begin{tabular}{cccccc}
\hline & Game N & Mean & $\begin{array}{c}\text { Std. } \\
\text { Deviation }\end{array}$ & $\begin{array}{c}\text { Std. Error } \\
\text { Mean }\end{array}$ \\
\hline Liking of new member & first & 59 & 5.15 & 3.18 & .41 \\
& second 49 & 4.91 & 3.41 & .49 \\
\hline
\end{tabular}

Table 5.7.3: Comparison of the discrimination measure for the two games. 


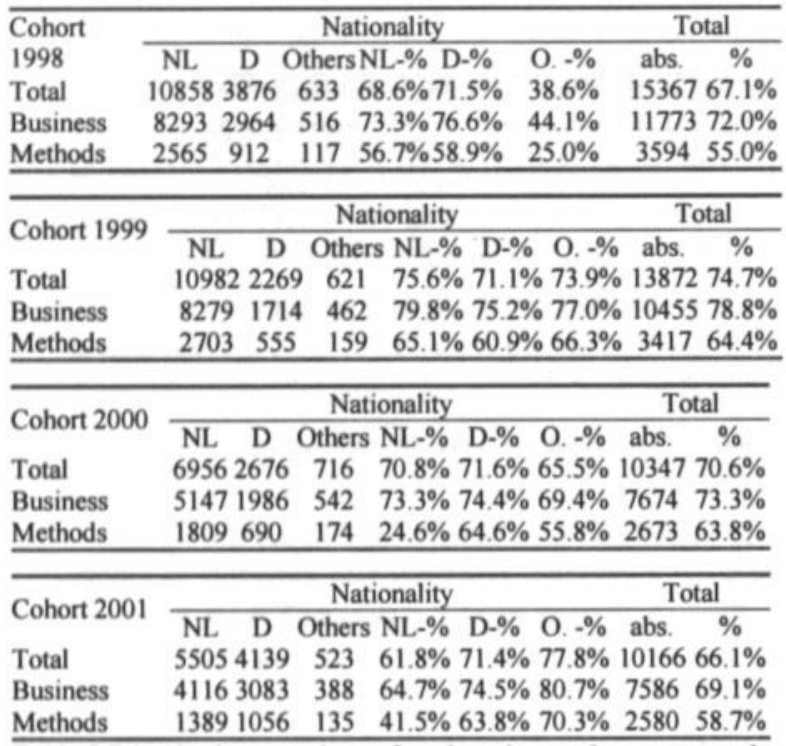

Table 5.7.4: Absolute numbers of study points and percentages in relation to all possible study points reached after one year, IB. Source: http://www fdewb.unimaas. $\mathrm{nl} / \mathrm{miso} / \mathrm{index} . \mathrm{htm}$.

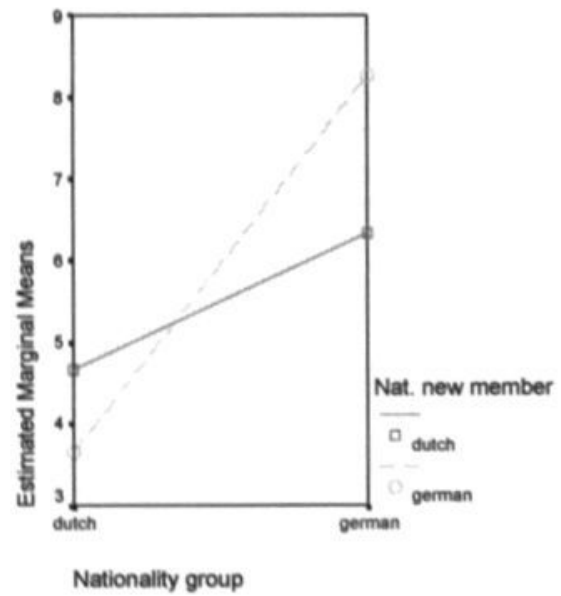

Figure 5.7.1: Graphical demonstration of the interaction effect for liking of new members, homogeneous groups. 


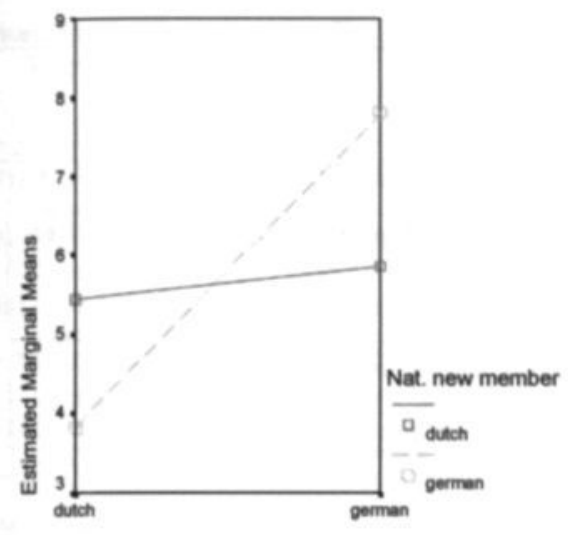

Nationality of group

Figure 5.7.2: Graphical demonstration of the interaction effect for liking of new members, including heterogeneous groups.

\section{Appendix II: 'Discrimination measure'}

Dear Student,

We were asked by a student, Jaap vanVelde (Andreas Mueller), who will be tutoring the Management Game next year, whether he could participate this year to get an impression about how it works. He did not do it as a student as he then studied in Rotterdam (Koeln). As I do not want to put him into a group, which feels really uncomfortable about it, I would like to ask you to indicate your preference below.

We chose this procedure, as we do not want to spend a whole lot of time on discussing this question in the team, and to make sure that everybody dares to indicate his real opinion. This could be difficult when asked in front of the group. As the game is important for you and you want to feel good in your team, such a change should not be made against the will of some team-members.

You can give this page to the tutor without a name on it, only with the group-number. We will check the preferences of the groups and then choose a group for him.

Please fill this in right now, so that he can participate from tomorrow on and you can go on with the gamel Thank you!

Group number:

Just indicate your preference by making a cross at that point on the line which represents it best! I rather do not watt Jaap in my group 


\section{Appendix III : Questionnaire game 1}

Date: June 2001

Please use the answer-scale below for the next question:

\begin{tabular}{|c|c|c|c|c|c|c|}
\hline $\begin{array}{c}\text { Very } \\
\text { unimportant }\end{array}$ & Unimportant & $\begin{array}{c}\text { Neither } \\
\text { unimportant nor } \\
\text { important } \\
1\end{array}$ & $\mathbf{2}$ & \multicolumn{2}{|c|}{ Important } & \multicolumn{2}{c|}{ Very important } \\
\hline 1) How important is it for you to do well in this game? & 1 & 2 & 3 & 4 & 5 \\
\hline
\end{tabular}

Please use the answer-scale below for the following questions:

\begin{tabular}{|c|c|c|c|c|}
\hline Very uncertain & Uncertain & $\begin{array}{c}\text { Neither uncertain } \\
\text { nor certain }\end{array}$ & Certain & Very certain \\
1 & 2 & 3 & 4 & 5 \\
\hline
\end{tabular}

2) How certain are you that you are personally doing well in this game?

3) How certain are you that your firm will be doing well in this game?

4) How certain are you about the measures your firm has to take to do well in this game?

5) How certain are you that you will succeed with your strategy?

6) How certain are you that you will reach your personal aims during this game?

\begin{tabular}{|l|l|l|l|l|}
\hline 1 & 2 & 3 & 4 & 5 \\
1 & 2 & 3 & 4 & 5 \\
1 & 2 & 3 & 4 & 5 \\
1 & 2 & 3 & 4 & 5 \\
1 & 2 & 3 & 4 & 5 \\
\hline
\end{tabular}

For the next question, please use the answer-scale below:

\begin{tabular}{|c|c|c|c|c|}
\hline Very weak & Weak & $\begin{array}{c}\text { Neither weak nor } \\
\text { strong }\end{array}$ & Strong & $\begin{array}{c}\text { Very strong } \\
5\end{array}$ \\
\hline
\end{tabular}

7) How strong is in your perception the competition between the firms? 
Please answer the questions below, using the following answer-scale:

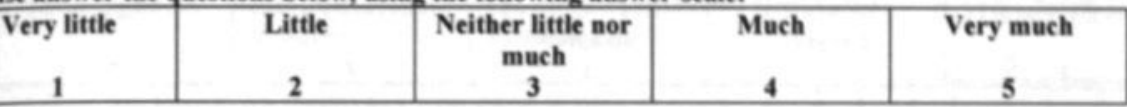

8) To what extent are personality clashes (conflicts) evident in your team?

9) How much tension is there among the members of your team?

10) How much jealousy or rivalry is there among the members of your team?

11) To what extent are there differences of opinion in your team?

12) To what extent are the arguments in your team task related?

\begin{tabular}{|l|l|l|l|l|}
1 & 2 & 3 & 4 & 5 \\
1 & 2 & 3 & 4 & 5 \\
1 & 2 & 3 & 4 & 5 \\
1 & 2 & 3 & 4 & 5 \\
1 & 2 & 3 & 4 & 5 \\
\hline
\end{tabular}

For the next questions, please use the following answer-scale:

No trust

1
Little trust

2
Moderate trust 3
High trust 4
Complete trust 5

To what extent do you trust your team members with regard to....

\begin{tabular}{l|l|l|l|l|l|}
\hline 13) Relying on their task-related skills and abilities & 1 & 2 & 3 & 4 & 5 \\
14) Sharing your personal beliefs & 1 & 2 & 3 & 4 & 5 \\
15) Relying on their interpersonal style/people skills at work? & 1 & 2 & 3 & 4 & 5 \\
16) Sharing your personal feelings? & 1 & 2 & 3 & 4 & 5 \\
17) Relying on their work-related judgments? & 1 & 2 & 3 & 4 & 5
\end{tabular}

Please use the answer-scale below for the following questions:

\begin{tabular}{|c|c|c|c|c|}
\hline $\begin{array}{c}\text { Hardly ever } \\
\mathbf{1}\end{array}$ & $\begin{array}{c}\text { Sometimes } \\
\mathbf{2}\end{array}$ & $\begin{array}{c}\text { Regularly } \\
\mathbf{3}\end{array}$ & $\begin{array}{c}\text { Often } \\
\mathbf{4}\end{array}$ & $\begin{array}{c}\text { Very often } \\
\mathbf{5}\end{array}$ \\
\hline
\end{tabular}

18) How often do people get angry while working in your team?

19) How often do the members of your team disagree about how things should be done?

20) How often do the members of your team disagree about which procedure should be used to do your work?

\begin{tabular}{|l|l|l|l|l|}
\hline 1 & 2 & 3 & 4 & 5 \\
1 & 2 & 3 & 4 & 5 \\
1 & 2 & 3 & 4 & 5 \\
\hline
\end{tabular}


Please rate your agreement with the following statements:

\begin{tabular}{|c|c|c|c|c|}
\hline Strongly disagree & $\begin{array}{c}\text { Moderately } \\
\text { disagree } \\
\mathbf{1}\end{array}$ & $\begin{array}{c}\text { Neither agree nor } \\
\text { disagree }\end{array}$ & Moderately agree & Strongly agree \\
\hline
\end{tabular}

21) I have positive feelings towards my team-members

22) I have confidence in my team-members

23) I feel comfortable depending on my team-members

24) I think the other team members perform well

25) I think that, with regard to decision making, I have more in common with members of this team than with members of other teams

26) I think that, generally speaking, I have more in common with members of this team than with members of other teams

27) I think that the members of this team are well suited to each other

28) I think it is right, in the view of my individual performance, that I am a member of this team

29) I find it pleasant to be a member of this team

30) I would like to continue working together with this team

31) I think it would be nicer in another team

32) I am glad that I ended up in this team

33) I have negative feelings about my team-members

34) I trust all memhers of my team equally moll

35) We do not seem to understand what one another is saying during our team discussions

36) The purpose of the team meetings we get involved in are clearly communicated

37) We really listen to one another and try to understand the feelings and points of view of each other

38) Each of us has the feeling that we can express ourselves on any issue at any time

39) We freely express our feelings and ideas in meetings

\begin{tabular}{|l|l|l|l|l|}
\hline 1 & 2 & 3 & 4 & 5 \\
1 & 2 & 3 & 4 & 5 \\
1 & 2 & 3 & 4 & 5 \\
1 & 2 & 3 & 4 & 5 \\
1 & 2 & 3 & 4 & 5 \\
1 & & & & \\
1 & 2 & 3 & 4 & 5 \\
1 & 2 & 3 & 4 & 5 \\
1 & 2 & 3 & 4 & 5 \\
1 & 2 & 3 & 4 & 5 \\
1 & 2 & 3 & 4 & 5 \\
1 & 2 & 3 & 4 & 5 \\
1 & 2 & 3 & 4 & 5 \\
1 & 2 & 3 & 4 & 5 \\
1 & 2 & 3 & 4 & 5 \\
1 & 2 & 3 & 4 & 5 \\
1 & 2 & 3 & 4 & 5 \\
1 & 2 & 3 & 4 & 5 \\
\hline
\end{tabular}




\begin{tabular}{|l|c|c|c|c|c|c|}
\hline Strongly disagree & \multicolumn{1}{c|}{$\begin{array}{c}\text { Moderately } \\
\text { disagree }\end{array}$} & $\begin{array}{c}\text { Neither agree nor } \\
\text { disagree }\end{array}$ & \multicolumn{2}{|c|}{ Moderately agree } & \multicolumn{2}{|c|}{ Strongly agree } \\
\hline 10) I talk up this team to my friends as a great group to work in & 1 & 2 & 3 & 4 & 5 \\
41) I am very committed to my team \\
$\begin{array}{l}\text { 42) I am willing to put a great deal of effort beyond what is } \\
\text { normally expected in order to help this team to be } \\
\text { successful }\end{array}$ \\
$\begin{array}{l}\text { 43) Individual team-members contribute equally to the } \\
\text { decisions made in the team }\end{array}$ \\
$\begin{array}{l}\text { 44) The formal leader of this team takes a leading role in } \\
\text { decision-making }\end{array}$ & 1 & 2 & 3 & 4 & 5 \\
\hline
\end{tabular}

To what extent do you agree that your team members...
45) Openly share information with you
46) Treat you on an equal basis with others
47) Can be trusted
48) Do things competently
49) Are receptive to what you have to say

\begin{tabular}{l|l|l|l|l|}
1 & 2 & 3 & 4 & 5 \\
1 & 2 & 3 & 4 & 5 \\
1 & 2 & 3 & 4 & 5 \\
1 & 2 & 3 & 4 & 5 \\
1 & 2 & 3 & 4 & 5 \\
\hline
\end{tabular}

Team Number:

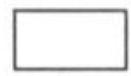

Personal Code (compulsory):

Id Number:
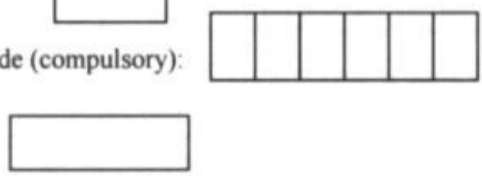

Thank you for filling in this questionnaire!
Personal Code:

- The first two letters of your mother's first name (e.g.: Maria: MA)

- The date of your birthday, in 2 digits (e.g.: January 08, 1980: 08)

- The first two letters of your place of birth (e.g. Amsterdam: $\mathbf{A M}$ )

Your personal code is: MA08AM 


\section{Appendix IV : Questionnaire game 2}

\section{Dear Student,}

This is the second questionnaire of a set of three. Please answer the questions honestly and hand in the completed form to your tutor together with the floppy disk on firm results.

Thank youl

Date: June 2002

Use the answer-scale below for the following questions:

\begin{tabular}{|c|c|c|c|c|c|c|}
\hline $\begin{array}{c}\text { Not at all } \\
1\end{array}$ & 2 & & \multicolumn{2}{|l|}{4} & \multicolumn{2}{|c|}{$\begin{array}{c}\text { Very much } \\
5 \\
\end{array}$} \\
\hline \multicolumn{2}{|c|}{$\begin{array}{l}\text { When playing the Management Game, to what extent } \\
\text { 1) do you understand it? } \\
\text { 2) are you certain about what is happening in the game? } \\
\text { 3) can you predict what is going to happen in the Management } \\
\text { Game? } \\
\text { 4) do you see the efforts of your team as related to the actual } \\
\text { team results? }\end{array}$} & $\begin{array}{l}1 \\
1\end{array}$ & $\begin{array}{l}2 \\
2\end{array}$ & $\begin{array}{l}3 \\
3\end{array}$ & $\begin{array}{l}4 \\
4\end{array}$ & $\begin{array}{l}5 \\
5\end{array}$ \\
\hline
\end{tabular}

For the next question, please use the answer-scale below:

\begin{tabular}{|c|c|c|c|c|}
\hline Very weak & Weak & Neither weak nor & Strong & Very strong \\
\hline 1 & 2 & 3 & 4 & 5 \\
\hline
\end{tabular}

5) How strong is in your perception the competition between the firms? 
Please rate your agreement with the following statements:

\begin{tabular}{|c|c|c|c|c|}
\hline Strongly disagree & $\begin{array}{c}\text { Moderately } \\
\text { disagree } \\
\mathbf{1}\end{array}$ & $\begin{array}{c}\text { Neither agree nor } \\
\text { disagree } \\
\mathbf{2}\end{array}$ & $\begin{array}{c}\text { Moderately agree } \\
\text { Strongly agree }\end{array}$ & $\mathbf{4}$ \\
\hline
\end{tabular}

\begin{tabular}{l}
\hline 6) In the team, we really listen to one another and try to \\
understand the feelings and points of view of each other
\end{tabular}
7) Each of us has the feeling that we can express ourselves on
any issue at any time
8) We freely express our feelings and ideas in meetings

Please use the answer-scale below for the following questions:

\begin{tabular}{|c|c|c|c|c|}
\hline Hardly ever & Sometimes & Regularly & Often & Very often \\
$\mathbf{1}$ & $\mathbf{2}$ & $\mathbf{3}$ & $\mathbf{4}$ & $\mathbf{5}$ \\
\hline
\end{tabular}

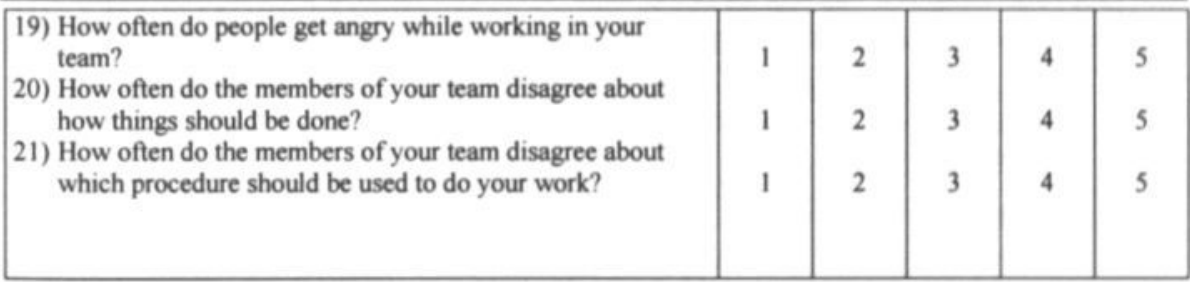


Please answer the questions below, using the following answer-scale:

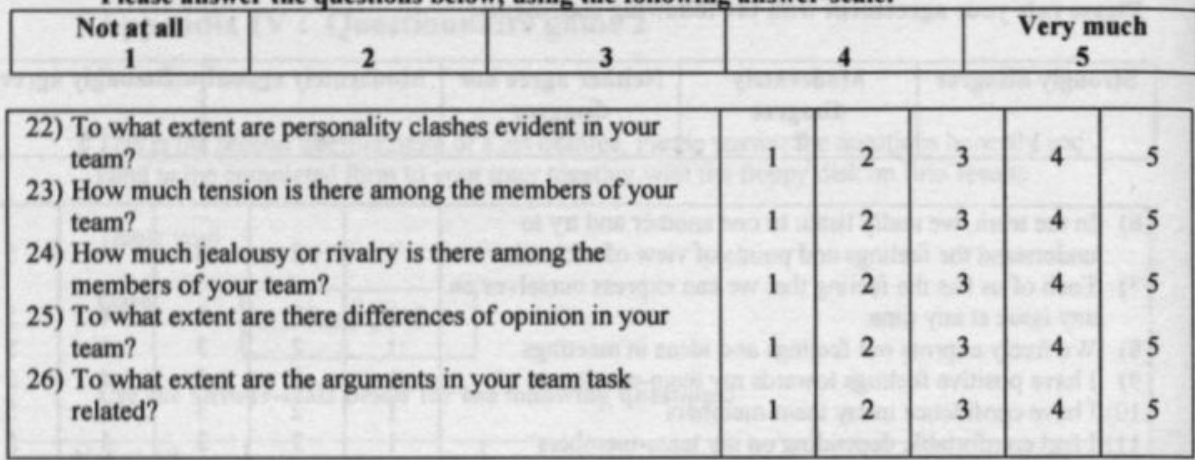

Please use the answer-scale below for the next question.

\begin{tabular}{|c|c|c|c|c|}
\hline $\begin{array}{c}\text { Very } \\
\text { unimportant }\end{array}$ & Unimportant & $\begin{array}{c}\text { Neither } \\
\text { unimportant nor } \\
\text { important }\end{array}$ & Important & Very important \\
\hline 1 & 2 & 3 & 4 & 5 \\
\hline
\end{tabular}

\begin{tabular}{|l|l|l|l|l|l|}
\hline How important is it for you & 1 & 2 & 3 & 4 & 5 \\
27) To do well as a firm in the game? & 1 & 2 & 3 & 4 & 5 \\
28) That your team outperforms the other teams? & & & & & \\
29) That everybody of your team contributes equally to the & 1 & 2 & 3 & 4 & 5 \\
success of the firm? & 1 & 2 & 3 & 4 & 5 \\
30) To have fun during team meetings? & 1 & 2 & 3 & 4 & 5 \\
31) To socialize during team meetings? & 1 & 2 & 3 & 4 & 5 \\
\hline 32) To get along well with your team members? & & & & \\
\hline
\end{tabular}

Finally, please indicate your

Team Number:

Id Number:

Personal Code:

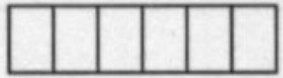

Personal Code:

- The first two letters of your mother' first name (e.g.: Maria: MA)

- The date of your birthday, in 2 digits (e.g.: January 08, 1980: 08)

- The first two letters of your place of (e.g. Amsterdam: AM)

Your personal code is: MA08AM 


\section{Is it efficiency wages? ${ }^{78}$}

\subsection{Introduction}

As has been shown in section 2.2, economics is providing a lot of empirical evidence that women earn lower wages than men, but the estimates of the wage gap vary widely with the variables included, the decomposition methods used, the database etc. (see also Stanley \& Jarell, 1998). In all estimates of the wage gap remains an unexplained part, which is most often interpreted as stemming from discrimination. Different economic and social-psychological theories are used to explain discrimination (e.g., Becker 1957, Phelps 1972, Arrow 1973, Fiske 1998), but one area of economic reasoning has so far hardly been used to explain the wage gap, even if it seems quite obvious that it could contribute to an explanation: efficiency-wage theory (see, however, Haagsma, 1993; 1995).

Efficiency-wage theory basically states that employers do not pay the lowest possible wage, but rather pay a higher wage (the lowest possible wage in efficiencyunits) to motivate workers to exhibit higher effort, to keep them from shirking or to attract better workers (see for an overview, e.g., Katz, 1986; Carmichael, 1990). In contrast to previous chapters, the focus here is therefore more on effort than on ability, even if versions of efficiency-wage concepts dealing with the attraction of better workers also look at ability.

In the framework of efficiency-wage theory, women's lower wages could be explained by assuming that efficiency wages for women are different or are believed to be different from those of men. This could be the case in two ways: First, firms could assume (wrongly or correctly) that women exhibit high effort/do not shirk already for low wages, i.e., lower wages than for men are sufficient to get the desired behavior from female workers. Second, firms could assume (again wrongly or correctly) that women do not react with more effort to higher wages (e.g., because they are not work/careeroriented anyway), so it might not be worth it to pay them high wages. In that case, firms would either employ women for the minimum possible wage or not employ them at all. Both assumptions can be based on stereotypes about women, describing in general men as more agentic, assertive, and work-oriented, and women as more communal, caring.

\footnotetext{
This chapter is based on a paper presented at the European ESA-meeting in Strasbourg, (2002) "An erroneous belief. Paying women lower wages does not pay".
} 
and family-oriented (for a review on gender stereotypes see, e.g., Deaux \& LaFrance 1998; Glick et al. 1995). It is quite probable that gender stereotypes play a role in the labor market, as gender is a very salient category and thus likely to lead to stereotype activation. In addition, typical sex roles, which relate to the work/family sphere, can have an impact on employers' beliefs about the reliability of women at the workplace: As women most of the times are the ones who take care of the children, they are believed to be absent from work or quitting work more often than men (for a discussion of gender differences in turnover rates see, e.g., Viscusi, 1980; Blau \& Kahn, 1981; Light \& Ureta, 1992; Sicherman, 1996). If the stereotypical assumptions about female reactions to incentives are on average correct, paying women lower wages is rational behavior from a profit-maximizing point of view. However, if the assumptions are not correct, lower wages for women might be economically inefficient, and a more 'psychological' explanation for such lower wages might then be useful.

In recent times, psychological research has put forward an explanation for women's lower wages, which can be interpreted as similar to an efficiency wage: The possibility that women are worse in negotiating for their salary and ask for lower wages than men in wage negotiations (see, e.g., Walters, Stuhlmacher \& Meyer, 1998; Pelham \& Hètts, 2001). In an efficiency-wage framework one could interpret this as women credibly promising the effort desired by the firm already for a lower wage than men.

In relation to the integrated framework developed in chapter 2 , this chapter analyses a special case of discriminatory behavior based on stereotypes in a very competitive setting. However, the experiment here does not look at the details of the mechanism in team-based firms, but rather looks more in general whether in a competitive situation individual subjects in the role of firms (employers) make 'discriminatory', i.e., lower, wage offers to women as compared to men. In addition, the experiment also wants to look at the supply side, to find out whether behavior of the 'discriminated' group explains the wage differences. If efficiency-wage effects based on different reactions of women and men towards incentives can be found, wage differences can be economically rational. If, however, the picture is such that purely (financial-) economic factors fall short in explaining the behavior found, psychological factors might be necessary to explain discriminatory outcomes. This would also be further evidence for the assumption that even in very competitive market settings, psychological factors can play a role, even without firms consisting of teams. 
The present chapter describes an experiment conducted to observe this in a controlled setting. The main reason for the choice of the experimental method is that it allows controlling for other factors influencing wages offered to or asked by women, which are hard to control for in other kinds of empirical studies. Furthermore, it allows for measuring effort level in a simple way, which is often very difficult in real-world settings (for a more elaborated discussion of the methodology-choice see section 2.8).

In an experimental double auction both workers and firms can make wage offers. This makes it possible to see whether women get offered lower wages than men, or whether they primarily ask for these lower wages. Further, it is possible to see whether efficiency-wage arguments in one or the other direction provide an explanation for the wage differential. In a laboratory setting stereotypes about female and male work attitudes should play a smaller role than in 'real life'. However, assuming with social psychology that stereotypes are universal and also work subconsciously (e.g. Fiske 1998), one should still expect to find an effect - and if an effect is found in a laboratory setting, the effect in real life is probably even bigger. Double auction markets are very competitive, and have been used before to test efficiency-wage theory, but also to test other related questions (see e.g. Davis \& Holt, 1993). In similar experimental double auctions with effort it was found that firms don't pay low wages, and that workers reciprocate with higher effort when getting higher wages (e.g., Charness, 1998; Fehr \& Falk, 1999; Hannan, Kagel, \& Moser 2002). In a double auction dealing with status differences between traders women made lower profits than men selling 'goods' - just like subjects who were assigned lower status (Ball, Eckel, Grossman \& Zame, 2001).

The rest of the chapter is organized as follows: First the hypotheses are formulated. In section 6.3, the experimental design is described in detail. Section 6.4 reports the results, and section 6.5 discusses them and concludes.

\subsection{Hypotheses}

In summary, the background of the experiment described here is a combination of efficiency-wage effects with knowledge about common stereotypes of women and men. Against this background, the following hypotheses can be formulated:

H1: Gender of employees influences wages, such that women receive lower wages (w) than men in the experimental double auction: $w_{f}<w_{m}$ 
H2: If HI holds, it holds, because efficiency-wage effects with respect to the effort (e) wage relationship are different for women and men: $e_{f}(w) \neq e_{m}(w)$. Two variants, based on common gender stereotypes, are possible:

H2a): As women are on average more cooperative than men, they exhibit high effort already for low wages: for given (low) $w$ that would imply $e_{f}>e_{m}$

H2b): As women are on average not very achievement-oriented, they never exhibit high effort, no matter how high the wage: for given (high) $w$ that would imply $e_{f}<e_{m}$

H3: If HI holds, it holds, because employers expect hypothesis two to hold whereas in fact it is not true (i.e., they discriminate against women as a group): $e_{f}^{e}(w) \neq e_{m}^{e}(w)$

H4: If HI holds, it holds, because women ask for lower wages, i.e., credibly promise already for a low wage to exhibit the amount of effort desired by the employer: $w_{f}^{a}(e)<$ $\boldsymbol{w}_{m}^{a}(e)$

\subsection{The experiment}

\subsubsection{Participants}

190 (94 male and 96 female) students of various faculties (the majority from economics and business) at Universitat Pompeu Fabra in Barcelona participated voluntarily, for performance-based payment, in the experiment.

\subsubsection{Design}

The design was adapted from Fehr \& Falk's (1999) study of underbidding on the labor market. The basic structure is a double auction with effort, with excess supply of workers (four firms and six workers). Each firm can contract only one worker per period. Workers and firms can make wage offers simultaneously, following an improvement rule. This means for workers, that each new wage offer always has to be lower than the last offer made before (by any of the workers). For firms, each new wage offer has to be higher than the last offer made before (by any of the firms). All offers can be accepted at any point in time, i.e., 'old' offers, which are still standing, can be accepted as well. No one has to accept an offer, i.e., firms do not have to conclude a contract with a worker, and workers do not have to conclude a contract with a firm. After three minutes trading is ended, no matter how many contracts are concluded. In a second step, when all contracts are concluded or three minutes are over, those workers who concluded a contract select 
an effort level (a percentage) which is costly for them and which determines their final payoffs and the profits of their firm. Workers can determine the effort level such that firms make losses ${ }^{79}$. Firms' payoffs $\pi_{\mathrm{F}}$ are calculated as follows:

$\pi_{f}=120^{\circ}$ percentage chosen by the worker - salary (between 20 and 120 ).

Workers' payoffs $\pi_{w}$ are calculated like this:

$$
\pi_{w}=\text { salary }- \text { costs of percentage }
$$

If a firm does not conclude a contract with a worker, the firm does not earn any money in that period. If a worker does not get a contract, he or she still gets twenty experimental guilders. Costs for the effort levels (percentages) are defined based on a design by Fehr, Kirchsteiger \& Riedl $(1998)^{80}$ (see table 6.1).

\begin{tabular}{|l|c|c|c|c|c|c|c|c|c|c|}
\hline Percentage & 10 & 20 & 30 & 40 & 50 & 60 & 70 & 80 & 90 & 100 \\
\hline Cost & 0 & 1 & 2 & 3 & 4 & 8 & 10 & 12 & 15 & 18 \\
\hline
\end{tabular}

Table 6.1: Percentages (effort-levels) and costs

The payoffs in experimental guilders were transformed in $€$-cent by multiplying them by two.

The experiment was run with all possible combinations of sexes of firms and workers, and everybody always knew the sex of workers and firms. In four treatments, workers and firms were always homogeneous with respect to sex, and in a last additional treatment, workers and firms were distributed into their roles randomly, regardless of their sex. This resulted in five different treatments, with four resp. three (in the mixed treatment) sessions of each treatment. Ten periods of the auction with effort were played in each session.

\begin{tabular}{||l|l|l|l|l|}
\hline \multirow{4}{*}{ Sex worker } & \multicolumn{4}{|c|}{ Sex firm } \\
\cline { 2 - 5 } & & male & female & mixed \\
\cline { 2 - 5 } & male & 4 & 4 & - \\
\cline { 2 - 5 } & female & 4 & 4 & - \\
\cline { 2 - 5 } & mixed & - & - & 3 \\
\hline
\end{tabular}

Table 6.2: Number of sessions for each constellation.

\footnotetext{
"The earnings function for the firms differed from that used by Fehr \& Falk (1999) in that the function used here allows that firms make losses.

" This cost function for effort levels was used to make the results of this experiment comparable with other related studies, like Fehr \& Falk's (1999), which also use this function.
} 
In the following, the treatments will be referred to mentioning always the sex of the worker first, and then the sex of firm.

\subsubsection{Procedure}

The experiment was conducted computerized in the experimental laboratory of Universitat Pompeu Fabra. A program, which Fehr \& Falk had used in one of their earlier double-auction experiments, was adapted. The experiment was programmed in zTree (Fischbacher 1999).

Upon arrival, students were divided into the two roles of firms and workers. In the sex-homogeneous sessions and the mixed sessions, this was done randomly. All participants gathered in front of the lab and drew lots with either "firm" or "worker" on it. Then, 'firms' and 'workers' entered the lab separately. In sex-heterogeneous sessions, this constituted the basic experimental manipulation: Before starting the experiment, the experimenter said loudly outside of the lab, in front of all participants "The girls (boys) come with me now, you are firms!" When the "firms' were seated, he brought the remaining subjects into the laboratory and said loudly when entering "The boys (girls) are workers". Then, worker/firm instructions were distributed (see appendix). Participants in the experiment were seated separately in front of their computers and could 'not see the other participants' screens. Subjects read the instructions and completed one page of exercises to test their understanding of the payoff structure. From the moment students were seated in their cubicles, no communication was allowed and questions about the experiment were only answered privately. While participants filled in the exercises the experimenter watched them carefully and pointed them to mistakes to secure understanding of the payoff structure of the experiment. When everybody had completed the exercises, the auction started. Ten periods of the double auction with effort were played. At the end of the experiment, subjects filled in a short questionnaire collecting demographical data. They were paid privately, based on what they earned in the experiment plus $€ 5$,- show-up fee. If subjects in the role of firms made losses they had to back it up with their show-up fee.

\subsection{Results}

The analysis was done on different levels of aggregation. Most hypotheses were tested using data from period one only, as behavior in later periods is not truly independent from what happened in earlier periods. Some analyses, however, use data 
over all periods, especially to show differences in trends over time for the treatments. If this is the case it will be indicated. Average wages are calculated only for workers/firms who had concluded a contract. Because of the relatively small number of independent observations, non-parametric Mann-Whitney-U tests were used to compare treatments. Correlational analyses are done using Pearson correlation coefficients, as linear relationships were assumed. However, as especially in the case of effort and wage normal distributions cannot be assumed, Spearman's rank correlations were calculated here as well. For significance testing holds the same as in previous chapters - marginally significant ( $10 \%$-level) results are reported, but have to be interpreted cautiously. If not otherwise reported, significance testing has been done two-tailed.

In the following, the results are presented by hypothesis, followed by a description of some exploratory results. A final section deals with the mixed sessions separately.

\subsubsection{Hypothesis I}

Hypothesis 1 was fully confirmed: Keeping sex of the firm constant, there is a significant difference in average wage (aggregated on session-level) in period one between female and male workers: Females earn significantly less than males $(Z=-2.21$, as. sig. $=.014$ (one-tailed)). Figure 6.1 shows box plots of average wages in period one. The dark line represents the median; the box represents $50 \%$ of the data, the lower boundary being the $25^{\text {th }}$ percentile and the upper boundary the $75^{\text {th }}$ percentile. The vertical lines represent values within 1.5 box lenghts from the upper or lower edge of the box; values further away are defined as outliers (up to 3 box lengths from the edges) or extreme values. Figure 6.2 shows the development of the average wage over all periods per constellation. 
middle-category exhibit slightly higher effort, and only those in the highest wage category really exhibit high effort levels.

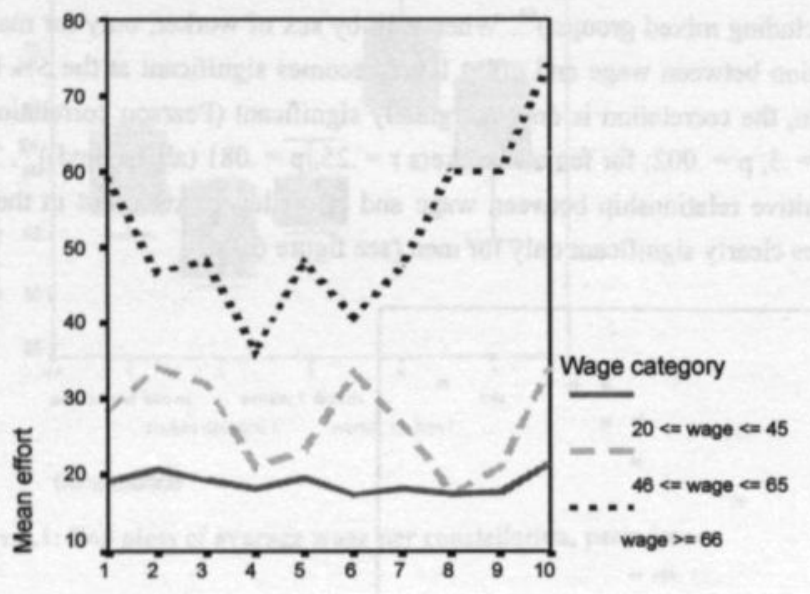

Period

Figure 6.4: Development of mean effort per wage category (mixed session included).

Women exhibit high effort only very seldom, in all wage categories. This could, however, be the result of women getting paid less than men in each category. To test for this, U-tests per wage category were calculated.

\begin{tabular}{ccccccc}
\hline Wage category & $\begin{array}{c}\text { Sex of } \\
\text { worker }\end{array}$ & N Mean wage & Std. Dev. & Z & $\begin{array}{c}\text { As. Sig (2- } \\
\text { tailed) }\end{array}$ \\
\hline $20<=$ wage $<=45$ & Male & 4 & 32.25 & 5.19 & -.70 & .484 \\
& Female & 18 & 34.56 & 7.9 & & \\
$46<=$ wage $<=65$ & Male & 19 & 54.95 & 4.25 & -1.42 & .156 \\
& Female & 12 & 53.17 & 4.59 & & \\
Wage $>=66$ & Male & 8 & 82.5 & 18.32 & -1.63 & .104 \\
& Female & 1 & 69.0 & & & \\
\hline
\end{tabular}

\section{Table 6.3: Mann-Whitney-U test of difference in wages between male and female workers per wage category (period one, individual-level data).}

Tables 6.3 and 6.4 show that only one woman is in the highest wage category in period one, while most women are in the lowest wage category. This is very different for men - only four men are in the lowest category, most men are in the middle category and 
still eight are in the highest category. This means that one can only restrictedly interpret an U-test between men and women for the highest and the lowest category, because of a very small $\mathrm{N}$ in one of the groups each time. In none of the wage groups, the test finds a significant difference between men and women - neither for wage level nor for effort level.

\begin{tabular}{ccccccc}
\hline Wage category & $\begin{array}{c}\text { Sex of } \\
\text { worker }\end{array}$ & N Mean effort & Std. Dev. & Z & $\begin{array}{c}\text { As. Sig (2- } \\
\text { tailed) }\end{array}$ \\
\hline $20<=$ wage $<=45$ & Male & 4 & 32.5 & 28.72 & -1.30 & .193 \\
& Female & 18 & 16.11 & 13.35 & & \\
$46<=$ wage $<=65$ & Male & 19 & 32.11 & 23.23 & -1.32 & .188 \\
& Female & 12 & 23.33 & 24.98 & & \\
Wage $>=66$ & Male & 8 & 76.25 & 31.59 & -1.63 & .104 \\
& Female & 1 & 10 & & & \\
& &
\end{tabular}

Table 6.4: Mann-Whitney-U test of difference in effort between male and female workers per wage category (period one, individual-level data).

It seems as if male and female firms expected that male workers would react positively to higher wages, whereas female workers would not. However, only one firm tried to pay a high wage to a female worker, which means, the question whether women do not react to high wages with more effort cannot be answered with these data. As table 6.4 shows, men also only change effort when moving to the highest wage category, and in this category there is only one woman.

\subsubsection{Hypothesis 4}

Mann-Whitney-U-tests are calculated for wages asked by women and men, to see whether women ask for lower wages or just get offered lower wages. All offers made in the first period were aggregated for firms and workers separately for this analysis. It was found that offers of firms in the first period are significantly lower when workers are women than when workers are men $(Z=-2.94$, as. sig. $=.003)$. On the other hand, workers of both sexes do not differ in the wages they ask for $(Z=-1.47$, as. sig. $=.141)$. Looking at the development over periods, from period four to six inclusively we also find a difference in the offers of the workers by sex - women obviously learn very quickly that they cannot ask for high wages and adjust their offers. The result is a significantly higher wage asked by male workers than by female workers from period four to six. The offers of firms remain significantly different for male and female workers until period four. 


\subsubsection{Other results}

For the four homogeneous constellations, over all periods together, a KruskalWallis test (Chi-Square $=33.41, \mathrm{p}=.000$ ) reveals a significant difference in the number of trades between the constellations. Figure 6.5 shows clearly that the female-maleconstellation resulted in a strongly decreasing number of trades down to zero until the end of the session, while in all other constellations, number of trades varied only between three and four (all possible trades). Testing for sex of firms res. workers, there is a significant difference in average number of trades (over all periods) between male and female workers: when workers are male, there are on average more trades over all periods than when workers are female $(\mathrm{Z}=-3.86, \mathrm{p}=.000)$ - which can also be seen in figure 6.5.

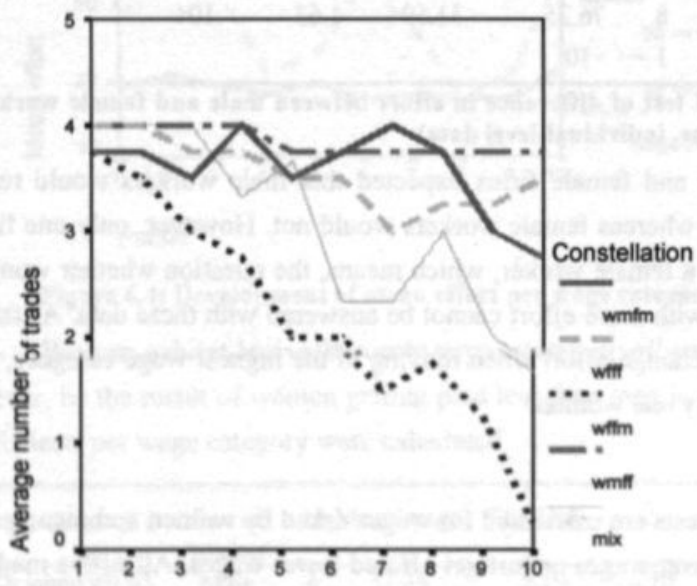

Period

\section{Figure 6.5: Development of number of trades per constellation.}

Male and female firms differ significantly with respect to number of trades as well: Female firms trade more $(Z=-3.97, p=.000)$. In fact, male firms often stopp trading at all when they make losses, whereas some female firms go on trading until the last period.

The pattern in the mixed group lies somewhere between the other groups - trades go down as well, not as completely as for male firms and female workers, but stronger 
6 Is it efficiency wages?

than in all other conditions (see below for an explanation). Basically in all those constellations where firms make losses, trading goes down over the periods.

A result that could provide an explanation for women's lower effort is that the difference between offers of firms and wages asked by the workers is higher for female workers than for male workers $(\mathrm{Z}=-2.21$, as. sig. $=.027)$. A t-test to see whether this discrepancy differed significantly from zero was calculated for all constellations. It showed that in the male-male-condition, in the male-female-condition, and in the mixed condition discrepancy is not significantly different from zero in period one. In both conditions with female workers, discrepancy is significantly different from zero. This means, only when workers are male or there are at least males among the workers, this discrepancy does not become significant (see also figure 6.6).

\begin{tabular}{lcrccc}
\hline Variable Constellation & $\mathrm{t}$ & $\mathrm{df}$ & $\begin{array}{c}\text { Sig. } \\
\text { (2-tailed) }\end{array}$ & $\begin{array}{c}\text { Mean } \\
\text { Difference }\end{array}$ \\
\hline \multirow{5}{*}{ Discrepancy } & Mm & 1.02 & 3 & .384 & 4.74 \\
& Ff & 5.23 & 3 & .014 & 18.21 \\
& Fm & 7.38 & 3 & .005 & 18.98 \\
& Mf & 2.32 & 3 & .103 & 10.27 \\
& Mix & .94 & 2 & .447 & 7.57 \\
\hline
\end{tabular}

Table 6.5: T-test of difference from zero (test-value $=0$ ) for discrepancy, for all constellations separately (period one per session).

Table 6.6 shows that men also ask more from female firms than from male firms (and get offered more from female firms than from male firms), but these effects are not significant. There are also no significant differences depending on sex of the firm in neither firm offers, the wages asked by workers nor discrepancy between wages asked and offered. 


\begin{tabular}{ccccccc}
\hline \multirow{2}{*}{ Constellation } & & N & Minimum & Maximum & Mean & Std. Deviation \\
\hline \multirow{3}{*}{$\mathrm{mm}$} & Offers by firms & 4 & 37.67 & 57.50 & 48.22 & 8.20 \\
& Offers by workers & 4 & 31.58 & 68.63 & 52.96 & 16.86 \\
& Discrepancy & 4 & -6.08 & 13.80 & 4.74 & 9.33 \\
\hline \multirow{3}{*}{$\mathrm{ff}$} & Offers by firms & 4 & 27.00 & 45.00 & 35.58 & 9.24 \\
& Offers by workers & 4 & 44.75 & 66.30 & 53.79 & 9.18 \\
& Discrepancy & 4 & 9.13 & 24.30 & 18.21 & 6.97 \\
\hline \multirow{3}{*}{$\mathrm{fm}$} & Offers by firms & 4 & 22.92 & 43.33 & 35.31 & 8.95 \\
& Offers by workers & 4 & 48.13 & 60.00 & 54.29 & 5.03 \\
& Discrepancy & 4 & 12.79 & 25.21 & 18.98 & 5.14 \\
\hline \multirow{3}{*}{$\mathrm{mf}$} & Offers by firms & 4 & 48.17 & 75.00 & 57.94 & 11.76 \\
& Offers by workers & 4 & 62.89 & 72.86 & 68.20 & 4.71 \\
& Discrepancy & 4 & -2.14 & 18.07 & 10.27 & 8.85 \\
\hline \multirow{2}{*}{ mix } & Offers by firms & 3 & 41.25 & 68.75 & 51.56 & 14.99 \\
& Offers by workers & 3 & 49.00 & 66.13 & 59.13 & 8.98 \\
& Discrepancy & 3 & -6.50 & 21.46 & 7.57 & 13.98 \\
\hline
\end{tabular}

Table 6.6: Descriptive statistics for offers by firms, offers by workers and discrepancy between them for all constellations separately (period one, per session).

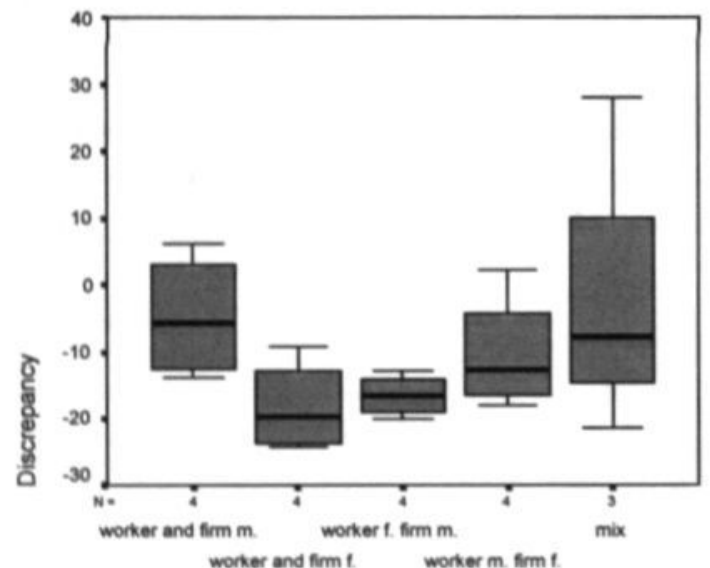

Constellation

Figure 6.6: Box plots of discrepancy between wage asked for and offered per constellation. 
Over all constellations, effort and discrepancy are negatively correlated $(r=-.6, p$ $=.003$ (1-tailed; for period one only) $)^{83}$, i.e., the higher the discrepancy, the lower the effort (excluding the mixed category it remains $r=-.58, p=.01)^{84}$. A regression analysis over all constellations for period one, using wage and discrepancy as explanatory variables for effort, confirms the importance of discrepancy between wage asked by the worker and offered by the firm for the effort level chosen. The complete linear regression model becomes significant $\left(R^{2}=.51\right.$, adjusted $R^{2}=.44$, sig. $\left.=.004\right)$, and both explanatory variables become significant as well, but 'discrepancy' is the more important one, as the beta-weight shows. Excluding the mixed sessions, the complete model remains significant (adj. $\mathrm{R}^{2}=.41$, sig. $=.014$ ), but only the beta-weight for 'discrepancy' remains significant. Discrepancy seems to be even more important than wage level in the determination of effort level.

\begin{tabular}{ccrrrr}
\hline & \multicolumn{4}{c}{ Std. Error Beta } & \multicolumn{1}{c}{ Sig } \\
\hline Includ. mixed sessions (Constant) & 14.04 & 1.04 & .315 \\
Wage & .24 & .40 & 2.18 & .045 \\
Discrepancy & .31 & -.49 & -2.68 .016 \\
Without mixed sessions (Constant) & 16.54 & & .91 & .379 \\
Wage & .28 & .40 & 1.95 & .073 \\
Discrepancy & .39 & -.47 & -2.25 & .042 \\
\hline
\end{tabular}

Table 6.7: Regression of wage and discrepancy on effort (period one).

\subsubsection{The mixed sessions}

In general, tendencies in the mixed sessions taken together are somewhere inbetween the homogeneous sessions - as one should expect. However, the mixed sessions varied in their composition, as table 6.8 shows - and this did not remain without an effect.

\begin{tabular}{ccccc}
\hline Session & Male firms & Female firms & Male workers & Female workers \\
\hline 17 & 2 & 2 & 1 & 5 \\
18 & 3 & 1 & 5 & 1 \\
19 & 1 & 3 & 2 & 4 \\
\hline
\end{tabular}

Table 6.8: Sex-composition of the mixed sessions.

\footnotetext{
${ }^{8}$ Spearman's rank correlation rho $=-.50, p=.014$.

"Spearman's rank correlation rho $=-.53, \mathrm{p}=.017$.
} 
One could assume that differences between these sessions were similar to the differences between the homogeneous sessions. More specifically, session 18 could be expected to be similar to the male-male sessions, while session 17 and 19 should be more similar to sessions with only female workers. It is of course not possible to compare the three sessions statistically, as the number of independent observations is too small. Still, an exploratory analysis, looking at descriptive statistics, was done to get an impression whether tendencies are as expected (see also table 6.9). The assumptions are partly confirmed - session 18 is more similar to an all-male session, while the other two sessions tend more towards male-female or all female sessions. This holds especially with respect to firm offers. However, session 17 seems to be strange in that the average wage is quite high, but still effort is low. Looking at the offers firms made, it becomes clear that in session 17 the discrepancy between wages offered by firms and wages asked by workers was high in the beginning - even if wages contracted in the end were not that low.

\begin{tabular}{|c|c|c|c|c|c|c|}
\hline Session & & $\mathrm{N}$ & Minimum & Maximum & m Mean & Std. Dev. \\
\hline \multirow{4}{*}{17} & Offers by firms & 3 & 25 & 70 & 44.67 & 23.03 \\
\hline & Offers by workers & 8 & 39 & 100 & 66.13 & 22.81 \\
\hline & Wage & 4 & 39 & 80 & 62.25 & 17.52 \\
\hline & Effort & 4 & 10 & 50 & 20.00 & 20.00 \\
\hline \multirow{3}{*}{18} & Mfers oy ifrms & $4^{\prime}$ & 60 & $75^{-}$ & 68.75 & 6.29 \\
\hline & Offers by workers & 4 & 55 & 69 & 62.25 & 6.08 \\
\hline & Wage & 4 & 60 & 70 & 67.25 & 4.86 \\
\hline \multirow{5}{*}{19} & Effort & 4 & 10 & 100 & 50.00 & 42.43 \\
\hline & Offers by firms & 4 & 25 & 70 & 41.25 & 20.16 \\
\hline & Offers by workers & 6 & 29 & 110 & 49.00 & 30.85 \\
\hline & Wage & 4 & 30 & 50 & 38.75 & 8.54 \\
\hline & Effort & 4 & 10 & 30 & 20.00 & 11.55 \\
\hline
\end{tabular}

Table 6.9: Descriptive statistics for offers by firms, offers by workers, wage, and effort for the mixed sessions separately (period one).

Discrepancy is lowest for session $18(-6.5)$ - here workers even asked less than the firms offered them - and as a consequence, effort is highest. Discrepancy is highest in session 17 (21.46 compared to 7.5 in session 19), but there is no difference in average effort between session 17 and session 19 - however, wages are very low in session 19 from the beginning on. Thus, the comparison of the three mixed groups shows the same tendencies as the analysis of the homogeneous data. Even if no statistical analysis is 
possible, this can carefully be taken as a confirmation of the results from the homogeneous sessions. It also shows that it might not even be necessary to make gender salient to get such effects.

\subsection{Discussion}

To summarize, it was possible to show in a double auction in the laboratory that women earn lower wages than men. Secondly, the findings show that women's behavior might have a partial influence on their wages. Women, however, are not too modest, but might be a bit less inclined to reciprocate higher wages with higher effort than men. However, this result has to be interpreted carefully, because firms determined wages, and firms did not make offers to women in the same wage range as to men. Furthermore, the experiment also points to a new explanatory factor for differences in effort next to the absolute wage: The difference between the wage asked by the worker in the beginning and the wage offered by the firm.

Men and women pay lower wages to women in the experiment, and they do this independently from what men and women ask for wages in the role of the worker. Male and female firms basically behave the same. However, from the second period on women indeed ask for lower wages. One experience of high discrepancy between the wage asked for and the wage offered by the firm seems to be sufficient for women to adapt their offers downwards. But, both men and women reciprocate, i.e., men and women react to low wages with low effort levels and to higher wages with higher effort levels - which made firms loose money in the experiment. Women did not get the chance to reciprocate higher wages with higher effort.

Only in the male-male-constellations firms do not make losses. In all other constellations on first sight the pattern looks like a striking lack of fairness concerns: Workers go up with their effort levels when they get higher wages, but most of the time they stay low enough to let firms make losses. Taking a closer look at the differences between the four conditions, one possible explanatory variable is recognized: Only in conditions with male workers discrepancy between the average wages firms offered and workers asked for was low, i.e., not significantly different from zero. This indicates that in all other conditions, where workers got offered lower wages than they thought they should get, this was basically seen as an unfair action in the beginning. This evoked a reaction that hurt the firms. Even in the condition with female firms and male workers, discrepancy is very close to significantly different from zero, because male workers ask 
very high wages from female firms. This could explain why even here firms made losses. Firms, which made losses, rationally responded with stopping to trade - even if adjustment took some time, as figure 6.5 showed. This confirms the economic intuition that firms do not make losses if they are able to avoid it.

One could argue that the wages asked for and offered in part are strategically determined, but this does not counteract the argument that these asks and offers were lower when workers were female than when they were male. There is also a problem of path-dependency, as even if one looks only at period one, the improvement rule implies that the first offer made determines all other offers. Again, this does not change the finding that these paths led to lower average offers made to women than to men.

What does this tell us for real-life situations and for further research? First, it shows that there is some kind of wage discrimination against women - either individual discrimination or group discrimination. Unfortunately, this experiment could not test whether this is based on wrong stereotypical perceptions of women or on correct estimates of their behavior, as women were not equally represented in all wage categories. If it were based on correct estimates of female behavior, it would of course be rational behavior by firms and thus 'only' constitute individual (statistical) discrimination. If it were based on wrong perceptions, it would constitute group discrimination as well.

Secondly, if employers think that they can make higher profits by paying women lower wages, they are wrong - at least in the experiment it did not work out for them: It lead to lower effort by women and very low or even negative profits by firms. This seems to be a vicious cycle: Women are paid lower wages, as a consequence they exhibit lower effort, employers see their stereotypes confirmed - and, as happened in the experiment, they rationally stop to employ women at all. This is also evidence against the mechanism Becker (1957) proposes for reducing discrimination, because it is assumed to work via lower wages for women in initial periods - not considering that this might lead to 'negative' reactions by the women.

Thirdly, the experiment shows that it is not mainly the absolute wage level, which determines effort levels, but the discrepancy between starting offers of firms and workers. It is not the case in this experiment that women ask for lower wages, they just get them - and therefore, they are in a situation of high discrepancy between their own idea, what wage they would like to get, and the wages firms want to pay them. Men, on the other hand, ask women to pay them more than they ask from other men, also leading 
to a discrepancy between wage offers. Thus, seemingly expectations - whether right or wrong - both sexes have about each other lead to discrepancies in wage offers, and to bad outcomes for firms and workers. Women in this experiment quickly adapt their expectations - but they also adapt their behavior to the low wages. It does not seem very unrealistic that women with a good education in the 'real world' are confronted with a discrepancy between their own wage expectations and what firms are willing to pay them. Meng (2002) found that female economists in their first job (in male occupations) earn lower wages than their male counterparts with the same education. This does not change with time; instead, the gap rather grows. Although Meng's study does not provide data about expectations of men and women for their first wage, a study by Blau and Ferber (1991) indicates that expectations women and men have about their starting salaries are quite similar - at least in the US.

Further research should test how women do react to high wages, i.e., whether it is a wrong or a correct expectation that it does not make sense to pay them higher wages because they don't react with higher effort (see, e.g., Major, McFarlin, \& Gagnon (1984) for evidence in the latter direction). Therefore, experiments should be done were women are confronted with high wages as well, for example using pre-programmed wage offers by firms, or the strategy method (a method which asks about reactions to all possible behaviors of the other party).

It would also be interesting to see what happens when employers can choose between women and men as workers in one auction, and whether they would stop trading with women and continue only with men if possible. In the mixed sessions reported here it was not possible to make an offer explicitly to female or male workers only. Unfortunately, it is difficult to do such an experiment, because if subjects are explicitly asked to choose between male and female workers, one runs the danger of getting only socially desirable behavior.

There might be another explanation for the differences in wage offers, different from an efficiency-wage effect: It could also be that emotional reactions, i.e., certain 'gut feelings' influence the wages offered to women - which is also proposed by the integrated model described in chapter 3. This, together with the question what expectations employers have, should be analyzed in further research. Again, this is not that easy, because asking about expectations might lead to socially desirable behavior, as people become aware of the topic at hand. However, if in the context of an experimental market subjects are asked about their expectations with respect to the effort-choice, and 
gender is introduced in the way it has been done here, such an effect can probably be avoided. Asking about 'gut feelings' might be more difficult, but there are measures like the "Implicit Association Test" (Greenwald et al. 1998), which have proven quite useful in measuring e.g. prejudice against blacks.

To conclude, the experiment gives an indication why discriminatory outcomes for women on the labor market might continue to exist even - or especially if it is possible to pay them lower wages than men, and how the perception that women are less productive than men can be confirmed by women's reactions to firm's behavior. In relation to the integration proposed, the experiment here showed that in highly competitive situations subjects in the role of firms discriminate against women, but also evoke reactions by doing so, which can confirm the 'stereotype'. As already discussed, the results here could follow from the influence of 'emotional' reactions (i.e., prejudice) rather than from cognitive ones, as there was no long thinking before the first offers were made. This would be evidence for a part of the integrated model not tested more in detail so far, the emotional aspect. However, these results also show that the 'economic' effect of competition might not work well, not only because of counteracting psychological forces, but also because of the reactions of the discriminated group. This is an aspect so far excluded from the analyses of this thesis, which is obviously very important to consider. Future developments of the integrated model should try to incorporate these reactions, as models of feedback effects in economics already do (e.g., Arrow, 1973; Coate and Loury, 1993; Farmer \& Terrell, 1996). 


\section{Appendix: Instructions translated into English ${ }^{85}$}

\section{You are a worker}

\section{General instructions for workers}

You will participate in a scientific research about the labor market. Please, read the instructions carefully. During the experiment your earnings are calculated in an artificial unit, called Gulden. At the end of the experiment

\section{one Gulden is worth $2 \epsilon$ - cent}

Your earnings will be paid to you in $€$ at the end of the experiment.

The 10 participants have been divided in two groups, worker (6) and firms (4). You can see whether you are a worker or a firm in the upper-right part of this page. The labor market consists of 10 days of trading, and one trading day consists of two parts:

1" part: In the first part, salaries are negotiated. Firms can offer salaries to the workers, and workers can, at the same time, propose salaries. Each side of the market can accept offers or asks of the other side. After 3 minutes, the second part starts.

$2^{\text {al }}$ part: Now the workers which have accepted an offer or whose offers have been accepted choose a percentage following certain rules. This is done following precisely specified rules and affects the earnings of the workers and the firms.

Overall there are 10 days of exchange. For participation in the experiment you will receive a show-up fee of 5 Euros plus the money you will earn during the trading.

Please note: During the experiment, no communication among the participants is allowed. Please, use the computers only following the instructions. If you do not follow these rules, you have to leave the experiment.

${ }^{85}$ Te original, Spanish instructions can be received upon request. 


\section{Information about the functioning of the labor market}

2) At each trading day the computer opens the labor market anew.

3) In the first part of the market firms can make salary offers to workers. At the same time, the workers can also propose wages.

Each worker can accept one of the offers of the firms and each firm can accept one of the offers of the workers. It is not obligatory to make or accept offers. The offers and asks have to be made in accordance with the following rules:

a. For you as a worker the rule is that your ask has to be always lower than the last ask made by a worker which has not been accepted.

b. For the firms the rule is that the offer, which it makes, has to be higher than the highest of the offers made by a firm that has not been accepted up to this moment.

c. All salaries between 20 and 120 are allowed (both inclusive). Your proposition of a salary has to be entered in the screen you see below.

\section{[screen shot I]}

The first column contains the field "your salary proposition". To make a salary proposition, first write your proposition in this field. This has always to be a number between 20 and 120 . In the following click with your mouse in the field "propose". Your salary proposition will appear in the second column below the title "Propositions of the workers". Note please that your proposals have to be always lower than the last proposal of a worker, which has not been accepted. You can make as many salary proposals as you whish.

All proposals of workers will appear in the second screen ordered by size. If many propositions are made and the column is "filled", you will only see the last propositions made.

Simultaneously, there will appear a scroll bar at the right side of the column. With the help of this scroll bar you can see all the propositions of workers. To do this, you have to move the scroll bar with the mouse.

In the third column you can see the salary offers of the firms. These appear ordered by size below the title "offers of firms". If many salary offers are made and the column is filled, you will only see the last wage offers made in the column "offers by firms". Simultaneously, there will appear a scroll bar at the right side of the column. With the help of this scroll bar you can see all the offers of firms. Therefore, you have to move the scroll bar with the mouse.

3) A salary contract is made when a salary has been agreed upon. This happens when a worker accepts an offer of a firm or a firm accepts the proposition of a worker.

You can always choose and accept one of the offers of the firms. If you want to accept the offer of a firm, you first have to choose with a mouse-click one of the offers in the third column, "offers of firms". If you want to accept it, click with the mouse on the field "accept". In this moment, you have closed a salary contract with the firm that has made this offer.

Always when a salary proposal has been accepted, this salary will appear in the fourth column below the title "salary contracts". If you have accepted a salary offer of a firm or a firm has 
accepted a salary proposition of you, the salary, which you have agreed upon will also appear in the fourth column below the title "your salary".

No worker is obliged to accept an offer or to make proposals. Each firm can close a salary contract with each worker and the other way round. You can accept any offer of a firm. At the same time, each firm can accept any of your salary proposals. At each day of trade a firm and a worker can close a maximum of one salary contract. This means, when on a trading day a salary contract has been closed between a firm and a worker, these two partners cannot close other salary contracts at this day of trade. When you have accepted an offer of a firm or one of your proposals of salary has been accepted, all your salary propositions and all salary offers of the firm with which you have closed the salary contract will disappear automatically. This implies that these salary-propositions and offers disappear in the second and third column and are no longer available.

Overall, firms and workers have exactly 3 minutes for making offers and proposals and accepting them. In the upper row of the screen you can see the remaining time. If time is over, no offers or propositions can be made anymore, and no salaries can be accepted.

4) No firm knows with which worker it has closed a salary contract and vice versa.

5) After 3 minutes the second part starts. Now the workers, which have closed a salary contract, have to choose a percentage. With this decision these workers influence their earnings and the earnings of "their" firms. This percentage is only communicated at the firm with which the contract was concluded. No other worker and no other firm learn the size of the chosen percentage. 


\section{How to calculate you earnings at one day of trade?}

1) If you have not concluded any salary contract, you will receive 20 Gulden at this day of trade.

2) If you have accepted an offer of a firm or a proposition of you has been accepted by a firm, you will receive the according salary. You have to subtract from this salary the costs of the percentage you have chosen.

3) You chose the percentage by choosing a number between 10 and 100 of the table you can see below. Number 10 is the lowest percentage; 20 a little bit higher percentage etc., and 100 the highest percentage.

The lower the percentage you choose, the lower are the costs for you. In the table you can see the costs, which correspond to each percentage.

Table of percentages that can be chosen and the corresponding costs.

\begin{tabular}{|l|c|c|c|c|c|c|c|c|c|c|}
\hline Percentage & 10 & 20 & 30 & 40 & 50 & 60 & 70 & 80 & 90 & 100 \\
\hline Cost & 0 & 1 & 2 & 3 & 4 & 8 & 10 & 12 & 15 & 18 \\
\hline
\end{tabular}

4) If you have closed a salary contract, your earnings in Gulden are calculated in the following way:

\section{Earnings $=$ Salary - Costs of percentage}

In general, the rule is: The higher your salary and the lower the costs of your percentage, the higher your earnings. 


\section{How are the earnings of the firms in a day of trading calculated?}

1) Each firm receives from the organizers 120 Gulden per trading day, which the firm can use to pay salaries. These 120 Gulden are multiplied by the percentage you have chosen. If for example you choose a percentage of 90 , the firm receives 90 percent of 120 Gulden, i.e., 108 Gulden. If for example you choose a percentage of 40 , the firm receives 40 percent of 120 Gulden, i.e., 48 Gulden. From this amount the "salary", which your firm "pays" to you is subtracted. The result are the earnings of the firm in Gulden:

\section{Earnings of the firm in Gulden: $[120 *$ (percentage/100)] - salary}

In general, the rule is: The higher the percentage and the lower the salary, the higher are the earnings of the firm.

It is possible that a firm makes losses. If a loss occurs, the firm has to pay this from the gains made or from the show-up fee.

2) If a firm does not close a salary contract, it does not have any earnings during that day of trading.

Note: The earnings of all workers and all firms are calculated following the same rule. Each firm receives at each day of trading 120 Gulden. The table of costs is the same for all workers. This is known to all participants. Each firm can, therefore, calculate the earnings of "its" worker at a trading day and each worker can calculate the earnings of "its" firm.

Your own earnings and the earnings of "your" firm are shown to you after each day of trade on the earnings screen. 
6 Is it efficiency wages?

\section{How to enter your percentage?}

The entering of your percentage is done using the following screen:

\section{[screen-shot 2]}

In the first row you can again see all salary contracts of this day of trading. In the second row you can see below the title "your salary" the salary you receive.

Below you can see a table. In the first column of this table you can see below the title "percentage" all possible percentages, which you can choose. In the second and third column you can see the resulting earnings for you and "your" firm respectively, when you have chosen a certain percentage. The earnings, which are shown in this table, are valid only for the specific salary, which you have received in this day of trade.

Below the table you see the entrance field. Here you can enter your percentage now. Please note that only numbers between 10 and 100 in intervals of 10 are allowed. 


\section{An example to practice!}

1) Assume that the worker (you) accepts an offer of 30 Gulden and chooses in the second part a percentage of 100 .

a. What are the earnings of the firm?

b. What are the earnings of the worker?

2) Assume that the worker (you) accepts an offer of 30 Gulden and chooses in the second part a percentage of 20 .

a. What are the earnings of the firm?

b. What are the earnings of the worker?

3) Assume that the firm has accepted your proposal of 70 Gulden and at the same time you chose a percentage of 100 in the second part.

a. What are the earnings of the firm?

b. What are your earnings?

4) Assume that the firm has accepted your proposal of 70 Gulden and at the same time you chose a percentage of 120 in the second part.

a. What are the earnings of the firm?

b. What are your earnings?

Table of percentages that can be chosen and the corresponding costs.

\begin{tabular}{|l|c|c|c|c|c|c|c|c|c|c|}
\hline Percentage & 10 & 20 & 30 & 40 & 50 & 60 & 70 & 80 & 90 & 100 \\
\hline Cost & 0 & 1 & 2 & 3 & 4 & 8 & 10 & 12 & 15 & 18 \\
\hline
\end{tabular}

5) Assume that you did not accept a salary offer and none of your salary proposals has been accepted during the day. What are your earnings at this day?

a. What are your earnings?

6) 5) Assume that no offer of a firm has been accepted and that this firm has also not accepted any proposition of a worker during the day. What are the earnings of the firm this day?

a. What are the earnings of the firm?

7) Assume that the firm has accepted your proposal of 45 Gulden and at the same time you chose a percentage of 40 in the second part.

a. What are the earnings of the firm?

b. What are your earnings?

8) Assume that the firm has accepted your proposal of 45 Gulden and at the same time you chose a percentage of 90 in the second part.

a. What are the earnings of the firm?

b. What are your earnings? 
6 Is it efficiency wages?

\section{You are a firm}

\section{General instructions for firms}

You will participate in a scientific research about the labor market. Please, read the instructions carefully. During the experiment your earnings are calculated in an artificial unit, called Gulden. At the end of the experiment,

\section{one Gulden is worth $2 €$ - cent}

Your earnings will be paid to you in $€$ at the end of the experiment.

The 10 participants have been divided in two groups, workers (6) and firms (4). You can see whether you are a worker or a firm in the upper-right part of this page. The labor market consists of 10 days of trading, and one trading day consists of two parts:

$1^{\text {It }}$ part: In the first part, salaries are negotiated. Firms can offer salaries to the workers, and workers can, at the same time, propose salaries. Each side of the market can accept offers or asks of the other side. After 3 minutes, the second part starts.

$2^{\text {ad }}$ part: Now the workers which have accepted an offer or whose offers have been accepted choose a percentage following certain rules. This is done following precisely specified rules and affects the earnings of the workers and the firms.

Overall there are 10 days of exchange. For participation in the experiment you will receive a show-up fee of 5 Euros plus the money you will earn during the trading.

Please note: During the experiment, no communication among the participants is allowed. riease, use the computers only following the instructions. If you do not follow these rules, you have to leave the experiment. 


\section{Information about the functioning of the labor market}

1) At each trading day the computer opens the labor market anew.

2) In the first part of the market firms can make salary offers to workers. At the same time, the workers can also propose wages. Each worker can accept one of the offers of the firms and each firm can accept one of the offers of the workers. It is not obligatory to make or accept offers. The offers and asks have to be made in accordance with the following rules:

a. For you as a firm the rule is that your offer has to be always higher than the highest of the offers of firms, which has not been accepted up to this moment.

b. For the workers the rule is that the offer, which it makes has to be ask has to be always lower than the last ask made by a worker which has not been accepted. All salaries between 20 and 120 are allowed (both inclusive). Your offer of a salary has to be entered in the screen you see below.

\section{[screen shot 1]}

The first column contains the field "your salary offer". To make a salary offer, first write your offer in this field. This has to be always a number between 20 and 120. In the following click with your mouse in the field "offer". Your salary offer will appear in the second column below the title "Offers of the firms". Note please that your offers have to be always higher than the last proposal of a firm that has not been accepted. You can make as many salary offers as you whish.

All offers of firms will appear in the second screen ordered by size. If many offers are made and the column is "filled", you will only see the last offers made. Simultaneously, there will appear a scroll bar at the right side of the column. With the help of this scroll bar you can see all the offers of firms. Therefore, you have to move the scroll bar with the mouse.

In the third column you can see the salary propositions of the workers. These appear ordered by size below the title "propositions of workers". If many salary proposals are made and the column is filled, you will only see in the column "propositions by the workers" the last wage proposals made. Simultaneously, there will appear a scroll bar at the right side of the column With the help of this scroll bar you can see all the proposals of the workers. To do this, you have to move the scroll bar with the mouse.

3) A salary contract is made when a salary has been agreed upon. This happens when a worker accepts an offer of a firm or a firm accepts the proposition of a worker.

You can always choose and accept one of the proposals of the workers. If you want to accept the proposal of a worker, you first have to choose with a mouse-click one of the proposals of workers in the third column, "propositions of workers". If you want to accept it, click with the mouse on the field "accept". In this moment, you have closed a salary contract with the worker, which has made this offer.

Always when a salary proposal has been accepted, this salary will appear in the fourth column below the title "salary contracts". If you have accepted a salary proposal of a worker or a worker has accepted a salary offer of you, the salary, which you have agreed upon, will also appear in the fourth column below the title "your salary payment". 
No firm is obliged to accept a proposal or to make offers. Each firm can close a salary contract with each worker and the other way round. You can accept any proposition of a worker. At the same time, each worker can accept any of your salary offers. At each day of trade a firm and a worker can close a maximum of one salary contract. This means, when on a trading day a salary contract has been closed between a firm and a worker, these two partners cannot close other salary contracts at this day of trade. When you have accepted a proposal of a worker or one of your salary offers has been accepted, all your salary offers and all salary proposals of the worker with which you have closed the salary contract will disappear automatically. This implies that these salary-offers and propositions disappear in the second and third column and are no longer available.

Overall, firms and workers have exactly 3 minutes for making offers and proposals and for accepting them. In the upper row of the screen you can see the remaining time. If time is over, no offers or propositions can be made anymore, and no salaries can be accepted.

4) No firm knows with which worker it has closed a salary contract and vice versa.

5) After 3 minutes the second part starts. Now the workers, which have closed a salary contract, have to choose a percentage. With this decision these workers influence their earnings and the earnings of "their" firms. This percentage is only communicated to the firm with which the contract was concluded. No other worker and no other firm learn the size of the chosen percentage. 


\section{How are the earnings of the workers calculated at one day of trade?}

1) If a worker has not concluded any salary contract, he will receive 20 Gulden at this day of trade.

2) If a worker has accepted an offer of a firm, or a proposition of the worker has been accepted by a firm, he will receive the according salary. He has to subtract from this salary the costs of the percentage he has chosen.

3) The worker chooses the percentage by choosing a number between 10 and 100 of the table you can see below. 10 is the lowest percentage; 20 a little bit higher percentage etc., and 100 the highest percentage.

The lower the percentage the worker chooses, the lower are the costs for him. In the table you can see the costs, which correspond to each percentage.

Table of percentages that can be chosen and the corresponding costs.

\begin{tabular}{|l|c|c|c|c|c|c|c|c|c|c|}
\hline Percentage & 10 & 20 & 30 & 40 & 50 & 60 & 70 & 80 & 90 & 100 \\
\hline Cost & 0 & 1 & 2 & 3 & 4 & 8 & 10 & 12 & 15 & 18 \\
\hline
\end{tabular}

4) If the worker has closed a salary contract, his earnings in Gulden are calculated in the following way:

\section{Earnings of the worker $=$ Salary - Costs of percentage}

In general, the rule is: The higher the salary and the lower the costs of the percentage, the higher the earnings of the worker. 


\section{How are your earnings in a day of trading calculated?}

1) You receive from the organizers 120 Gulden per trading day, which you can use to pay salaries. These 120 Gulden are multiplied by the percentage "your" worker has chosen. If for example he chooses a percentage of 90 , you receive 90 percent of 120 Gulden, i.e., 108 Gulden. If for example he chooses a percentage of 40 , you receive 40 percent of 120 Gulden, i.e., 48 Gulden. From this amount the "salary", which you "pay" to the worker is subtracted. The result are your earnings Gulden:

\section{Earnings in Gulden: [120 * (percentage/100)] - salary}

In general, the rule is: The higher the percentage and the lower the salary, the higher are your earnings.

It is possible that you make losses. If a loss occurs, you have to pay this from the gains made or from the show-up fee.

2) If you do not close a salary contract, you do not have any earnings during that day of trading.

Note: The earnings of all workers and all firms are calculated following the same rule. Each firm receives at each day of trading 120 Gulden. The table of costs is the same for all workers. This is known to all participants. Each firm can, therefore, calculate the earnings of "its" worker at a trading day and each worker can calculate the earnings of "its" firm.

Your own earnings and the earnings of "your" worker are shown to you after each day of trade at the earnings screen. 


\section{An example to practice!}

1. Assume that you offer a salary of 30 Gulden and a worker accepts this offer and chooses in the second part a percentage of 100 .

a) What are your earnings?

b) What are the earnings of the worker?

2. Assume that you offer a salary of 30 Gulden and a worker accepts this offer and chooses in the second part a percentage of 20 .

a) What are your earnings?

b) What are the earnings of the worker?

3. Assume that you have accepted a proposal or a worker of $70 \mathrm{Gulden}$ and at the same time the worker chooses a percentage of 100 in the second part.

a) What are your earnings?

b) What are the earnings of the worker?

4. Assume that you have accepted a proposal or a worker of 70 Gulden and at the same time the worker chooses a percentage of 20 in the second part.

a) What are your earnings?

b) What are the earnings of the worker?

Table of percentages that can be chosen and the corresponding costs.

\begin{tabular}{|l|c|c|c|c|c|c|c|c|c|c|}
\hline Percentage & 10 & 20 & 30 & 40 & 50 & 60 & 70 & 80 & 90 & 100 \\
\hline Cost & 0 & 1 & 2 & 3 & 4 & 8 & 10 & 12 & 15 & 18 \\
\hline
\end{tabular}

5. Assume that you did not accept a salary proposals and none of your salary offers has been accepted during the day. What are your earnings at this day?

a) What are your earnings?

6. Assume that no proposal of a worker has been accepted and that this worker has also not accepted any offer of a firm during the day. What are the earnings of the worker this day?

a) What are the earnings of the worker?

7. Assume that you have accepted a proposal of a worker of 45 Gulden and at the same time the worker chose a percentage of 40 in the second part.

a) What are your earnings?

b) What are the earnings of the worker?

8. Assume that you have accepted a proposal of a worker of 45 Gulden and at the same time the worker chose a percentage of 90 in the second part.

a) What are your earnings?

b) What are the earnings of the worker? 


\section{Screen-shot 1:}

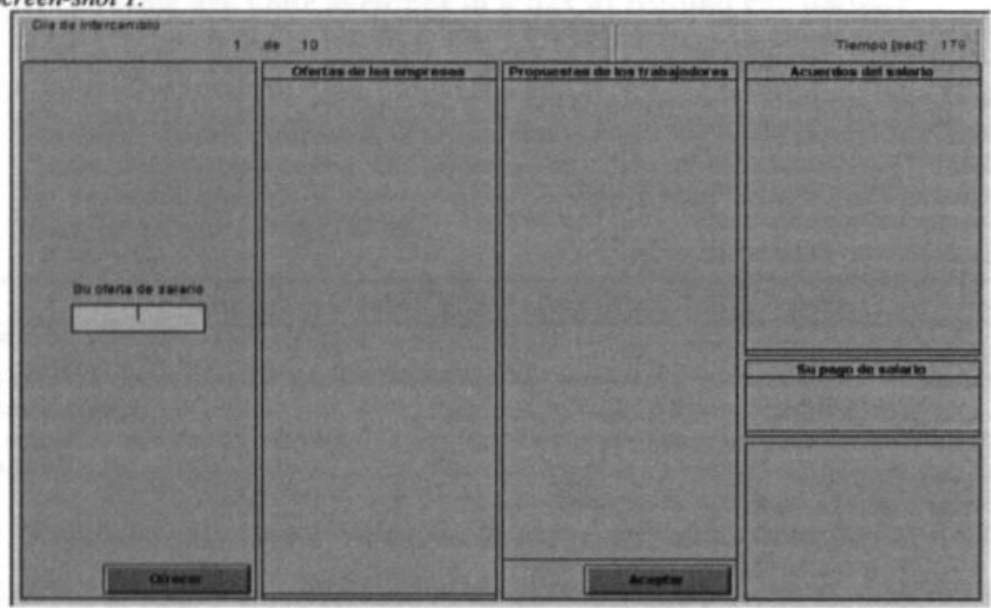

Screen-shot 2:

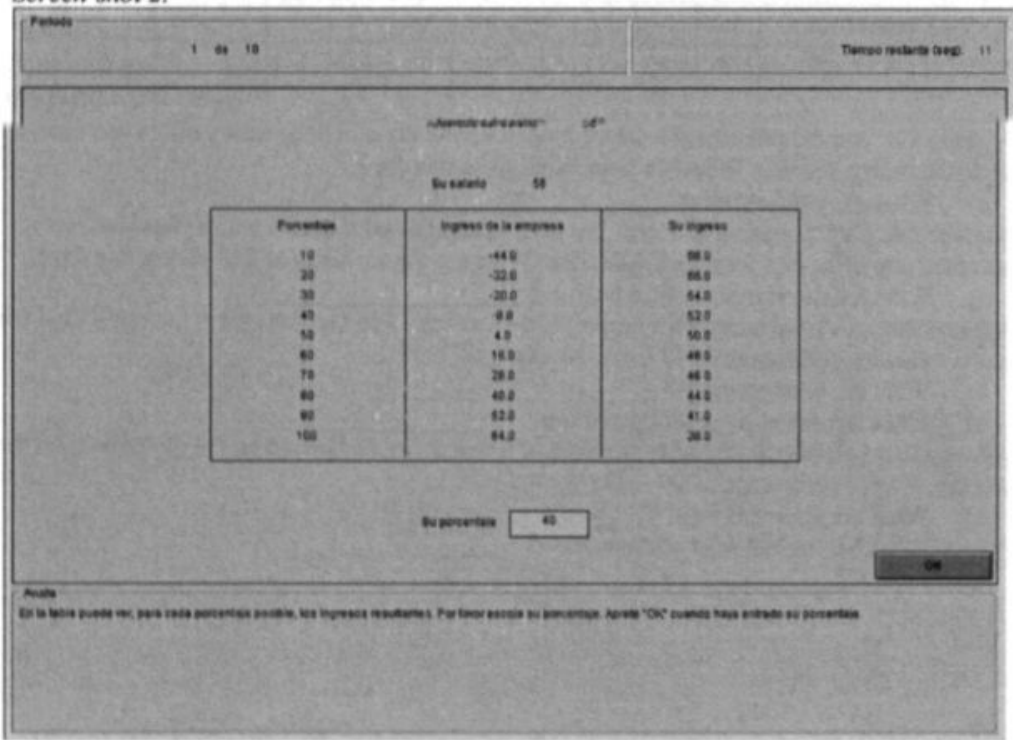




\section{Summary, general discussion, and conclusions}

\subsection{Introduction}

This last chapter summarizes the results of all chapters of the thesis and discusses them in the light of the whole project. The focus is on which questions could be answered, which questions could not be answered, and which new questions arose. First, the main aims of the thesis and the results of each of the chapters are summarized shortly. Secondly, these results are discussed. A general conclusion focuses on the scientific achievements of this research as well as on possible policy recommendations.

\subsection{Summary}

\subsubsection{The basic questions}

The main aims of this thesis were: (i) To get an overview of some related literature in economics and social psychology relevant for an analysis of discrimination in the labor market, (ii) to develop an integrated, testable theoretical model making use of both literatures, and (iii) to test at least part of the predictions of this model experimentally - to answer the basic questions whether an integration of economic and psychological approaches can explain discriminatory behavior in the labor market better than one approach alone; whether an integrated theoretical model can lead to new testable predictions; and finally, whether experimental tests can provide evidence for at least some of these predictions.

More in particular, a model was to be constructed which, by inclusion of the psychological concepts of prejudice, stereotypes, and identification, is able to explain and predict how discriminatory preferences may develop during a process of increasing competition. Predictions of this model were tested in experimental and quasiexperimental studies.

\subsubsection{Short summary of each chapter}

Chapter 1 provided an introduction into the topic of the thesis. The most prominent economic and psychological explanatory approaches of discrimination in the labor market were reviewed, and the aim and approach of this thesis were lined out and explained.

Chapter 2 gave an overview of some related literature on discrimination in economics and social psychology. First, empirical evidence on discrimination in the 
labor market was reviewed. Then it was shown that definitions of the core concepts differ within and between the disciplines, but also that there are some important similarities between disciplines. The differences in the use of the core concepts can pose a problem for the construction of an integrated model. Therefore, the chapter developed inclusive definitions of each of the concepts to be used in the further analysis.

The definition of stereotypes used in this thesis is taken from social psychology: Stereotyping is "...the unconscious or conscious application of (accurate or inaccurate) knowledge of a group in judging a member of the group" (Banaji \& Greenwald 1994, p. 54). The definition of prejudice is taken from social psychology as well: Prejudices are negative, affective attitudes towards all or most members of a certain group (cf. Pettigrew, 1997).

The definition of social identity was also taken from social psychology: Social identification is categorization in terms of and identification with groups one belongs to. The social identity is part of a person, in addition to the 'personal identity'. Groups one is part of and identifies with are called 'ingroups', in contrast to 'outgroups'.

The definition of self-relevant subjective uncertainty used is a combination of the concept of uncertainty social identity theory uses and 'economic' concepts of uncertainty: Self-relevant subjective uncertainty is defined as a subjective perception of ('objective') uncertainty accompanied by an aversive emotional state, which becomes stronger, the more self-relevant the source, and the higher the level of uncertainty is. It can pertain to all kinds of uncertainty, with growing strength: Other things being equal, risk evokes weaker feelings of subjective self-relevant uncertainty than ambiguity and fundamental uncertainty. The distinction between risk, as a situation with uncertainty about outcomes, ambiguity as a situation of uncertainty about probabilities, and fundamental uncertainty as uncertainty about things which cannot be known is adapted from Knight (1921) and Camerer \& Weber (1992).

It was more complicated to choose a definition of competition, which was applicable to the research questions here and could be related to standard economic definitions. The definition used conceptualizes competition as an activity performed by firms or other economic agents, who engage in this activity against each other, to reach certain goals.

Finally, an integrative framework was described which provides a starting point for the development of a formal integrated model focusing only on certain aspects of the 
7 Summary, general discussion, and conclusions

framework. Some methodological questions with respect to the approach of the research to be conducted were discussed at the end of the chapter.

Chapter 3 developed the integrated model formally. Social-psychological and economic insights were used to develop a model that explains how an increase in competition can induce employers to rely more on stereotypic perceptions and prejudices in their hiring, pay, and promotion decisions. This was based on the observation in social psychology that using stereotypic perceptions (for example, in personnel decisions) has certain benefits as well as costs (e.g., Fiske 1998). The most important benefit in this context is that, together with identification with an ingroup (e.g., men), the use of stereotypes and prejudices reduces subjective feelings of uncertainty, which can arise from competition. This psychological effect of strengthening competition works in the opposite direction of the economic income effect in the model of employer discrimination of Becker (1957). Both effects are integrated in the model. The model can explain the persistence and the rise of discrimination in the labor market in spite of and due to competitive pressure. This is reached by introducing stereotypes and prejudices as endogenous. The situation analyzed is one of team-structured firms, such that the model can explain employer and employee discrimination in such a setting. The model refrains from an analysis of the influence of specific contents of stereotypes. The content of stereotypes could also have implications for the relative strength of both effects. The question in what situations which effect is stronger, however, is an empirical question and remains open.

Chapter 4 provides a first step towards empirical testing of the integrated model. The experiments, using artificial groups, provided evidence for the idea that the psychological mechanisms influence 'discriminatory' behavior, depending on strength of competition, as the integrated model predicted. In all but the first experiment, a significant number of subjects used the - irrelevant - category information as basis for decision-making, and more so, when competition was stronger. Thus, the experiments showed that one effect of strengthening competition is that subjects make use of any 'information' available. This only holds when categories can be assumed to have at least some informational content, i.e., when they are constructed such that they can either vaguely be linked to the task subjects have to do, or a stereotype about them is developed. The psychological mechanism obviously determines that, when competition is strong, subjects believe more strongly in the 'informational' content of these categories. In this case, they statistically discriminate against the group which they think 
The experiments described in chapter 4 and the study using the business game gave some empirical evidence for the importance of psychological factors, which determine together with 'statistical' forms of discrimination the total amount of discrimination. As the empirical studies did not allow for testing all mechanisms assumed by the integrated model in detail, it is still not possible to say, how the interplay of economic and psychological factors exactly works, but certain tendencies became clear. What is clear now is that competition influences preferences, following the mechanisms predicted by social-identity theory. The stronger competition is perceived to be, the more people rely on all information they can find to make hiring decisions - even if this information is not seen as relevant when competition is weak. This results in a weak form of statistical discrimination, based on wrongly perceived productivity differences, which only appears when competition is strong.

Whether this leads to bias in favor of the ingroup or the outgroup, or to bias against stereotypically more or less productive groups, depends especially on beliefs about own performance and on the kind of categories. In the experiments with artificial categories, competition led to outgroup bias when subjects believed that their own group performed badly, and the outgroup might perform better. In the business game however, ingroup bias and in part even bias against the better performing group was found, most probably, because in 'real' situations people do not only look at productivity-related stereotypes, but other stereotypes and also prejudice play a role.

The integrated model does assume that emotional or prejudiced reactions towards the groups in question play a role. This was not tested explicitly in any of the experiments, but the results from the business game and also from the double auction can in part be interpreted as being the result of such emotional reactions, as ingroup bias resp. bias against women occurred obviously without being linked to (productivity-related) stereotypes.

The results of the double auction further reinforce the assumption that strong forces are at work when real categories are used, as even in this laboratory situation women get lower wage offers than men. The experiment also provides an additional argument against the working of the economic effect assumed in Becker's model: It does not consider possible reactions by the 'unfairly' paid workers. Chapter 6 showed that this is an important aspect, which should be incorporated in a model of discrimination in the labor market. Reactions from the supply side of the labor market are considered in the 
integrated framework, but not yet incorporated into the formal model. This could be a next step to take.

Some problems of the research were, as expected, related to the differences in levels of analysis between the two disciplines, but they could mainly be overcome relying on integrative definitions. More difficult to overcome were the operationalization and measurement problems. Not all constructs could be operationalized in a way that allowed for capturing those aspects of the model that should be captured. Measuring discrimination avoiding social-desirability problems worked out well in the experiment described in chapter 6 , but it turned out to be more difficult in the experiments of chapter 4. It is unclear in how far it was a problem in the business game. In the experiments in chapter 4 , it was not the social-desirable behavior directly which posed the problems, but rather the fact that the artificial categories, which were used to avoid social-desirability considerations, were not prone to make subjects identify strongly with them and were too 'empty' to be a good proxy of 'real' categories. In the business game, it is not clear in how far social desirability influenced Dutch and German students differently - but it could be an alternative explanation for the fact that German students were in general more willing to accept new members than Dutch students.

Especially the valid measurement of perceived subjective uncertainty turned out to be difficult in all (quasi-) experimental studies reported here. Several different, but related ways of measurrement were tried. In experiment 1 and 3 one very simple, direct question was used. Experiment 2 and 4 tried, not very successfully, to develop a more complex scale, being able to cover several aspects of the construct of uncertainty instead of directly asking participants how uncertain they were (with respect to their results). The aspects, which should be covered, were uncertainty about reaching specific team results, but also uncertainty about reaching personal goals. This was included because in such artificial situations it is very well possible that participants follow different aims than experimenters want them to follow - and thus, their uncertainty is not covered by asking about the aim they are supposed to have. This attempt at measuring a more complex construct of uncertainty was repeated in the first Business Game, using similar questions adapted to the specific situation. For the second Business game, the questionnaire had to be shortened. This was taken as a possibility to change the questions on uncertainty such that they covered a far broader concept of uncertainty, namely uncertainty about the 
game and about what happened in general (like in experiment $4^{86}$ ). This last operationalization comes closest to the one used by Mullin and Hogg (1998), but does not really cover the aspect of 'self-relevance'. It is important that further research develops a more reliable scale, which can be validated and then be used as a standard measure in related research.

The research around the integrated framework left many possibilities and also necessities for further research. First, one should further develop the experimental design such that the important variables of the integrated model can be measured and analyzed meaningfully. It should be extended to include the economic effect as well, building on but avoiding the caveats of the experiments reported here.

Secondly, testing of the predictions of the model in 'real-life' situations is necessary. The fact that only (quasi-) experimental results so far have been used to test the model - or rather parts of it - leads to problems of generalizability. Empirical data about hiring, workforce, and team composition should be collected, controlling for the competitive situation of firms and measuring identification and self-relevant uncertainty as well as stereotypes about groups.

The integrated model assumes that emotional reactions towards the groups play an important role in discriminatory behavior, and this has not directly been tested in the studies conducted up to now. Therefore, a next step would be to find ways to measure these emotional reactions and their influence on hiring behavior in different situations, for example making use of the Implicit Association Test (Greenwald et al, 1998).

Another important topic is that the current research assumed that mechanisms for all kinds of discrimination are the same. However, there might be differences based on the contents of stereotypes and the value-loading of emotional reactions. Therefore, a controlled comparison of different situations, e.g. gender discrimination, discrimination by nationality, race, or age, would be interesting to see in how far the mechanisms are universal, and policy recommendations can be the same for all kinds of situations. One could also extend the model such as to allow for the inclusion of specific contents of stereotypes if necessary.

\footnotetext{
"s It did not form a reliable scale here either, but unfortunately data collection for experiment 4 and the second management game were around the same time, such that this could not be taken into consideration.
} 


\subsection{Final Conclusion}

The thesis has shown that integrating economic and psychological theorizing focusing on the 'activity' aspect of competition can lead to interesting insights in the mechanisms of discrimination in the labor market. These insights can contribute to economic research, as they allow for explanations and predictions of the development of discriminatory behavior, which consider the fact that it is human beings who act in firms and on markets. It was shown that the assumption of endogenous stereotyping and use of prejudice leads to predictions different from those of standard economic models. The contribution to social-psychological research consists of introducing psychological variables in a formal way into a model describing a specific situation, discrimination in the labor market. This required more precise specifications of important psychological variables and the ways they interact with the environment and with each other. It allows proposing a relationship of cognitive and emotional variables with behavior in this specified setting, which can be tested empirically.

The empirical testing of the model showed that economic mechanisms of statistical discrimination and psychological mechanisms of social identification influence behavior jointly, and psychological forces are quite strong. This has implications for policy advice, because the recommendation from the standard economic model of employer discrimination so far was rather to count on the forces of competition in fighting discrimination. The integrated model and the experiments showed that this might often not lead to the desired results. Social psychology does only rarely give policy advice, which is in part a consequence of the many different detail results - to say it with Brewer's words: "The lack of integration of social-psychological research and theory relevant to the understanding of prejudice and discrimination makes it difficult to tell the story to the world" (1994, p. 316f.).

It is still too early to give policy advice based on the results from this interdisciplinary study. However, it can be said that politics should neither build only on the forces of competition, nor ignore them completely in fighting discrimination. It should also be kept in mind that even if stereotypes can be changed, there might be underlying emotional evaluations which are very strong and hard to change, and which will influence behavior especially when people are in situations of high self-relevant uncertainty. Reactions of the discriminated group can also not be ignored, which especially implies that the 'economic effect of competition' described by Becker will probably be counteracted by such reactions. 
7 Summary, general discussion, and conclusions

To conclude, the research showed that interdisciplinary research is always difficult, but that in particular (but not only) for the topic of this thesis it is a fruitful exercise to do. It can contribute to the development of both disciplines, and can help to achieve better-informed policy recommendations. 


\section{References}

Aigner, D.J. \& Cain, G.G. (1977), "Statistical Theories of Discrimination in Labor Markets". Industrial and Labor Relations Review, 30, 175-187.

Akerlof, G. A. (1970), "The Market for 'Lemons': Quality Uncertainty and the Market Mechanism". Quarterly Journal of Economics, 84, 488 - 500.

Akerlof, G. A. \& Kranton, R. E. (2000), "Economics and Identity". The Quarterly Journal of Economics, 115, 715-753.

Allport, G. W. (1954), The Nature of Prejudice, Reading, MA, Addison Wesley.

Altonji, J. G. \& Pierret, C. R. (1997), "Employer Learning and Statistical Discrimination". National Bureau of Economic Research Working Paper: 6279

Altonji, J.G. \& Blank, R.M. (1999), "Race and Gender in the Labor Market". In Ashenfelter, O. \& Card, D. (eds.), Handbook of Labor Economics, 3C, Amsterdam: Elsevier, 3143-3259.

Amir, Y. (1969), "Contact hypothesis in ethnic relations". Psychological Bulletin, 7I, 319-342.

Anderson, D. \& Shapiro, D (1996), "Racial Differences in Access to High Paying Jobs and the Wage Gap Between Black and White Women". Industrial and Labor Relations Review, 49, 273-86.

Anshel, M. H., Jamieson, J., \& Raviv, Sh. (2001), "Cognitive appraisals and coping strategies following stress among skilled competitive male and female athletes". Journal of Sport Behavior, 24, 128-143.

Arrow, K. J. (1973), "The Theory of Discrimination". In Ashenfelter, O. \& Rees, A. (eds.), Discrimination in Labor Markets. Princeton: Princeton University Press, 3-33. Ashenfelter, O. \& Hannan, T. (1986), "Sex Discrimination and Product Market Competition: The Case of the Banking Industry", Quarterly Journal of Economics, $101,149-173$.

Ashraf, J. (1996), "Is Gender Pay Discrimination on the Wane? Evidence from Panel Data, 1968 1989". Industrial and Labor Relations Review, 49, 537-546.

Baetge, J., Fischer, Th., \& Kunberger A. (1998), Management Information on The INTOP III Business Game. On the basis of INTOP by Thorelly, H. B., Graves, R. L., Howells, L. T., $6^{\text {th }}$ edition, Muenster. 
Baldwin, M. L. \& Johnson, W. G. (1996), "The Employment Effects of Wage Discriminatin Against Black Men". Industrial and Labor Relations Review, 49, 302316.

Ball, S., Eckel, C., Grossman, P. J. \& Zame, W. (2001), "Status in Markets". The Quarterly Journal of Economics, 116, 161-188.

Banaji, M. R. \& Greenwald, A. G. (1994), "Implicit Stereotyping and Prejudice". In Zanna, M. P. \& Olson, J. M. (eds.), The Psychology of Prejudice: The Ontario Symposium, 55-77.

Becker, G. (1957, 1971), The Economics of Discrimination. $1^{\text {st }}$ and $2^{\text {nd }}$. eds. Chicago: The University of Chicago Press.

Becker, G. S. (1993), "Nobel Lecture: The Economic Way of Looking at Behavior". Journal of Political Economy, 101, 385-409.

Bergmann, B. R. \& Darity, W. Jr. (1981), "Social Relations, Productivity, and Employer Discrimination". Monthly Labor Review, 104, 47-49.

Berk, J. B. (1999), "Statistical Discrimination in a Competitive Labor Market,", National Bureau of Economic Research Working Paper 6871.

Bertrand, M. \& Hallock, K. F. (2000), "The Gender Gap in Top Corporate Jobs".

National Bureau of Economic Research Warking Paner: 7931.

Betsch, T., Plessner, H., Schwieren, C. \& Gatig, R. (2001), "I Like it but I Don't Know Why: A Value Account Approach to Implicit Attitude Formation," Personality and Social Psychology Bulletin, 27, 242 - 253.

Biernat, M., Manis, M. \& Nelson, Th. E. (1991), "Stereotypes and standards of judgment". Journal of Personality and Social Psychology. 60, 485-499.

Biernat, M. \& Kobrynowicz, D. (1997), "Gender and race based standards of competence: Lower minimum standards but higher ability standards for devalued groups". Journal of Personality and Social Psychology, 72, 544-557.

Biernat, M., Vescio, Th. K. \& Manis, M. (1998), "Judging and behaving toward members of stereotyped groups: A shifting standards perspective". In Sedikides, C., Schopler, J. et al (ed.), Intergroup cognition and intergroup behavior. Mahwah, NJ, US: Lawrence Erlbaum Associates, 151-175.

Black, D, A (1995), "Discrimination in an Equilibrium Search Model". Journal of Labor Economics, 13, 309-333. 
Blau, F. D. (1998), "The Gender Pay Gap; Women's work and wages". In Persson, I. Y Jonung, Ch. (ed.), Research in Gender and Society, 2. London and New York: Routledge, 15-35.

Blau, F. D. \& Beller, A. H. (1988), "Trends in Earnings Differentials by Gender, 1971 1981". Industrial and Labor Relations Review, 41, 513-29.

Blau, F. D. \& Ferber, M. A. (1991), "Career Plans and Expectations of Young Women and Men: The Earnings Gap and Labor Force Participation". Journal of Human Resources 26, 581-607.

Blau, F. D. \& Ferber, M. A. (1992); Women's Work, Women's Lives: A Comparative Economic Perspective; By Women's work and women's lives: The continuing struggle worldwide, Boulder and Oxford: Westview Press, 28-44.

Blau, F. D., Ferber, M. A., and Winkler, A. E. (1998), The Economics of Women, Men, and Work, $3^{\text {rd }}$ edition, London: Prentice Hall.

Blau, F. D., Ferber, M., \& Winkler, A. (2002) The Economics of Women, Men, and Work, 4th edition, London: Prentice Hall.

Blau, F. D., \& Kahn, L. M. (1981), "Race and Sex Differences in Quits by Young Workers". Industrial and Labor Relations Review, 34, 563-77.

Blau, F. D. \& Kahn, L. M. (1994), "Rising Wage Inequality and the U.S. Gender Gap". American Economic Review, 84, 23-28.

Blau, F. D. \& Kahn, L. M. (1997), "Swimming Upstream: Trends in the Gender Wage Differential in the 1980". Journal of Labor Economics, 15, 1-42.

Blau, F. D. \& Kahn, L. M. (2000), "Gender Differences in Pay". Journal of Economic Perspectives, 14, 75-99.

Blau, F. D. \& Kahn, L. M. (2002), At home and abroad: U.S. labor market performance in international perspective. New York: Russell Sage Foundation.

Blaug, M. (2001), "Is Competition Such a Good Thing? Static Efficiency versus Dynamic Efficiency". Review of Industrial Organization, 19, 37-48

Bless, H., Clore, G. L, Schwarz, N. Golisano, V. et al. (1996), "Mood and the use of scripts: Does a happy mood really lead to mindlessness?" Journal of Personality and Social Psychology, 71(4), 665-679.

Bless, H. \& Schwarz, N. (1999), "Sufficient and necessary conditions in dual mode models: The case of mood and information processing". In Chaiken, Sh. \& Trope, Y. (eds.), Dual process theories in social psychology. New York, 423-440. 
Blinder, A. S. (1973), „Wage Discrimination: Reduced Form and Structural Estimates“. Journal of Human Resources, 8, 436 - 455.

Bloch, F. \& Rao, V. (1993), "Statistical Discrimination, Identity Selection, and the Social Transformation of Caste and Race". Brown University Department of Economics Working Paper: 93-15.

Bodenhausen, G. V. \& Wyer, R. S. (1985), "Effects of stereotypes in decision making and information processing strategies". Journal of Personality and Social Psychology, 48, 267-282.

Bodenhausen, G. V., Macrae, C. N. \& Garst, J. (1998), "Stereotypes in thought and deed: Social cognitive origins of intergroup discrimination". In Sedikides, C., Schopler, J. et al. (eds.), Intergroup cognition and intergroup behavior. Mahwah, NJ, US: Lawrence Erlbaum Associates, 311-335.

Bodenhausen, G. V. \& Moreno, K. N. (2000), "How do I feel about them? The role of affective reactions in intergroup perception". In Bless, H. \& Forgas, J. P. (eds.), The message within: The role of subjective experience in social cognition and behavior. Philadelphia, US: Psychology Press, 283-303.

Bodenhausen, G. V., Mussweiler, Th., Gabriel, Sh., \& Moreno, K. N. (2001), "Affective

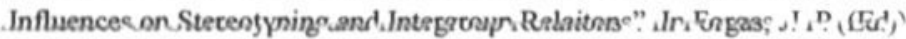
Handbook of Affect and Social Cognition. Mahwah, NJ, US: Lawrence Erlbaum Associates, 319-343.

Borjas, G. J. \& Bronars, S. G. (1989), "Consumer Discrimination and Self employment". Journal of Political Economy, 97, 581-605.

Bornstein, G., Mingelgrin, D. \& Rutte, Ch. (1996), "The effects of within group communication on group decision and individual choice in the assurance and chicken team games". Journal of Conflict Resolution, 40, 486-501.

Bowlus, A. J. \& Eckstein, Z. (1998), "Discrimination and Skill Differences in an Equilibrium Search Model". Centre for Economic Policy Research, Discussion Paper: 1859 .

Brandts, J. \& Charness, G. (2003), "Truth or Consequences: an Experiment". Management Science, 49, 116-130.

Brewer, M. B. (1979), "Ingroup bias in the minimal intergroup situation: A cognitive motivational analysis". Psychological Bulletin, 86, 307-324. 
References

Brewer, M. B. (1994), "The social psychology of prejudice: Getting it all together". In Zanna, M. P. \& Olson, J. M. (eds.), The psychology of prejudice: The Ontario symposium, 7. Hillsdale, NJ: Lawrence Erlbaum, 315-329.

Brickenkamp, R. (1962), Aufmerksamkeits Belastungs Test (Test d2), $1^{\text {st }}$ edition. Gottingen.

Bruggert, F., Schippers, J., et al. (1999), "The Relation Between the Relative Wage of Women and the Profitability of Firms: A Test of Becker's Employers' Discrimination Theory". Paper presented at the EALE conference 1999, Regensburg.

Camerer, C. (1998), "Bounded Rationality in Individual Decision Making". California Institute of Technology, Social Science Working Paper 1029. Pasadena, California. Camerer, C. \& Weber, M. (1992), "Recent Developments in Modeling Preferences:

Uncertainty and Ambiguity". Journal of Risk and Uncertainty, 5, 325-70

Carmichael, H. L. (1990), "Efficiency Wage Models of Unemployment - One View". Economic Inquiry, 28, 269-295.

Cattell, R. B. and Weiss, R. H. (1971), Grundintelligenztest Skala 3 (CFT 3). Braunschweig.

Charness, G. (1998), “Attribution and Reciprocity in a Simulated Labor Market: An Experimental Investigation". Mimeo, University of California, Santa Barbara.

Claire, Th. \& Fiske, S. T. (1998), “A systemic view of behavioral confirmation:

Counterpoint to the individualist view". In Sedikides, C., Schopler, J. et al. (eds), Intergroup cognition and intergroup behavior. Mahwah, NJ, US: Lawrence Erlbaum Associates, 205-231.

Clore, G. L., Schwarz, N., \& Conway, M. (1994), "Affective causes and consequences of social information processing". In Wyer, R. S. \& Srull, T. K., Handbook of Social Cognition, 1, 323-417, Hilldale NJ: Erlbaum.

Coate, S. \& Loury, G. (1993), "Will Affirmative Action Policies Eliminate Negative Stereotypes?". American Economic Review, 83, 1220-1242.

Comanor, W. S. (1973), "Racial Discrimination in the American Industry". Economica, 40, 363-378.

Cook, S. W. (1962), "The systematic study of socially significant events: A strategy for social research". Journal of Social Issues, 18, 66-84.

Corcoran, M. \& Duncan, G. J. (1979), "Work History, Labor Force Attachment, and Earnings Differences between the Races and Sexes". Journal of Human Resources, $14,3-20$ 
Damasio, A. R. (1994), Descartes' Error: Emotion, Reason, and the Human Brain. New York: Putnam.

Darley, J. M \& Fazio, R. H. (1980), "Expectancy confirmation processes arising in the social interaction sequence". American Psychologist. 35, 867-881.

Datta Gupta, N., Oaxaca, R. L. \& Smith, N. (1999), "Changes in the Gender Wage Gap: A Comparison Between the U.S. and Denmark". Presentation at the $11^{\text {th }}$ EALE Annual Conference.

Davis, D. D. \& Holt, C. A. (1993), Experimental Economics. Princeton, NJ: Princeton University Press.

Deaux K. \& LaFrance, M. (1998), "Gender". In Fiske, S. T., Gilbert, D. T., and Lindsay, G. (eds.), The Handbook of Social Psychology, Vol. 2, New York: McGraw Hill, $788-827$.

Dekker, H. (1999), Nationaal favoritisme, germanofobia en Europees burgerschap. Socialisatie van emoties. Utrecht: Onderzoekschool Arbeid, Welzijn en Sociaal Economisch Bestuur.

Dequech, D. (2001), "Bounded Rationality, Institutions, and Uncertainty". Journal of Economic Issues, 35, 911-929.

Dijker, A. J. \& Koomen, W. (1996), "Stereotyping and Attitudinal Effects under Time Pressure". European Journal of Social Psychology, 26, 61-74.

Doosje, B., Ellemers, N., \& Spears, R. (1999), "Commitment and intergroup behavior". In Ellemers, N., Spears, R. et al. (Eds), Social identity: Context, commitment, content. Oxford, England: Blackwell Science Ltd., 84-106.

Dovidio, J. F \& Gaertner, S. L. (1986), "Prejudice, discrimination, and racism: Historical trends and contemporary approaches". In Dovidio, J. F. \& Gaertner, S. L. (eds.), Prejudice, discrimination, and racism. San Diego, CA, US: Academic Press, 1-34.

Dovidio, J. F., Brigham, J. C., Johnson, B. T., \& Gaertner, S. L. (1996), "Stereotyping, Prejudice, and Discrimination: Another Look". In Stangor, C., Macrae, N., \& Hewstone, M. (eds.), Stereotypes and Stereotyping, New York: Guilford. 276-319 Eagly, A. H. \& Chaiken, S. (1998), "Attitude Structure and Function". In Fiske, S. T., Gilbert, D. T., \& Lindsay, G. (eds.), The Handbook of Social Psychology, Vol. I, New York: McGraw Hill, 269 - 322.

Ellemers, N., Spears, R. et al. (Eds.) (1999), Social identity: Context, commitment, content. Oxford, England: Blackwell Science Ltd. 
Ellsberg, D. (1961), "Risk, Ambiguity, and the Savage Axioms". Quarterly Journal of Economics, 75, 643-669

Ely, R. J. (1995), "The power in demography: Women's social constructions of gender identity at work". Academy of Management Journal, 38, 589-634.

Esses, V. M., Haddock, G. \& Zanna, M. P. (1994), "The role of mood in the expression of intergroup stereotypes". In Zanna, M. P. \& Olson, J. M. (eds.), The psychology of prejudice: The Ontario symposium, Vol. 7. Ontario symposium on personality and social psychology, Hillsdale, NJ, England: Lawrence Erlbaum Associates, 77-101.

Farmer, A. \& Terrell, D. (1996), "Discrimination, Bayesian Updating of Employer Beliefs and Human Capital Accumulation". Economic Inquiry, 34, 204-219.

Fazio, R. H. \& Towles Schwen, T. ( 1999), "The MODE model of attitude behavior processes". In Chaiken, Sh. \& Trope, Y. (Eds.), Dual process theories in social psychology. New York, US: Guilford Press, 97-116.

Fehr, E., Kirchsteiger, G., \& Riedl, A. (1998), "Gift Exchange and Reciprocity in Competitive Experimental Markets". European Economic Review, 42, 1-34

Fehr, E. \& Falk, A. (1999), "Wage Rigidity in a Competitive Incomplete Contract Market". Journal of Political Economy, 107, 106-134.

Fehr, E. \& Schmidt, K. M. (1999), “A Theory of Fairness, Competition, and Cooperation". Quarterly Journal of Economics, 114, 817-868.

Festinger, L. (1957), A theory of cognitive dissonance. Oxford, England: Row, Peterson. Fiedler, K. (1996a), "Explaining and simulating judgment biases as an aggregation phenomenon in probabilistic, multiple cue environments". Psychological Review, 103, 193-214.

Fiedler, K. (1996b), "Die Verarbeitung sozialer Informationen fur Urteilsbildung und Entscheidungen“. In Stroebe, W., Hewstone, M., \& Stephenson, G. M. (eds.), Sozialpsychologie. Eine Einfuehrung. Berlin/Heidelberg/New York: Springer, 143176.

Fiedler, K. (2000), "Beware of Samples! A Cognitive Ecological Sampling Approach to Judgment Biases," Psychological Review, 107, 659-676.

Fiedler, K., Kemmelmeier, M. \& Freytag, P. (1999a), "Explaining Asymmetric Intergroup Judgments through Differential Aggregation: Computer Simulations and Some New Evidence". European Review of Social Psychology, 10, 1 - 40. 
Fiedler, K., Walther, E., \& Nickel, S. (1999b), "The Auto Verification of Social Hypotheses: Stereotyping and the Power of Sample Size". Journal of Personality and Social Psychology, 77, 5-18.

Fischbacher, U. (1999), "z-Tree: Zurich Toolbox for Readymade Economic Experiments. Instructions for Experimenters". IEW Working Paper 21, University of Zurich.

Fiske, S. T. (1998), "Stereotyping, Prejudice, and Discrimination". In Fiske, S. T., Gilbert, D. T., and Lindsay, G. (eds.), The Handbook of Social Psychology, Vol. 2, New York: McGraw Hill, 357 - 414.

Fiske, S. T. \& Stevens, L. E. (1993), "What's so special about sex? Gender stereotyping and discrimination". In Oskamp, S. \& Costanzo, M. (Eds.), Gender issues in contemporary society. Claremont Symposium on Applied Social Psychology, Vol. 6. Thousand Oaks, CA, US: Sage Publications, 173-196.

Fortin, N. M. \& Lemieux, T. (1998), "Rank Regressions, Wage Distributions, and the Gender Gap". The Journal of Human Resources, XXXIII, 610-43.

Fox, C. R. \& Weber, M. (2002), "Ambiguity Aversion, Comparative Ignorance, and Decision Context”. Organizational Behavior and Human Decision Processes, 88, 476-498.

Friedman, D. \& Sunder, Sh. (1994), Experimental Methods. A Primer for Economists. Cambridge University Press.

Frisch, D. \& Baron, J. (1988), "Ambiguity and rationality". Journal of Behavioral Decision Making. I, 149-157.

Fuchs, V. R. (1986), "His and Hers: Gender Differences in Work and Income, 19591979". Journal of Labor Economics, Part 2, 4, S245-72

Fujii, E. T. \& Trapani, J. M. (1978), "On Estimating the Relationship between Discrimination and Market Structure". Southern Economic Journal, 44, 556- 567 Gaertner, S. L. \& Dovidio, J. F. (1986), "The aversive form of racism". In Dovidio, J. F. \& Gaertner, S. L. (eds.), Prejudice, discrimination, and racism. San Diego, CA, US: Academic Press, 61-89.

Gardner, R. C. (1994), "Stereotypes as consensual beliefs". In Zanna, M. P. \& Olson, J. M. (eds.), The psychology of prejudice: The Ontario symposium, Vol. 7. Ontario symposium on personality and social psychology, Hillsdale, NJ, England: Lawrence Erlbaum Associates, 1-31.

Gigerenzer, G. \& Selten, R. (eds.) (2001), Bounded rationality: The adaptive toolbox. Cambridge, MA, US: The MIT Press. 
Glick, P., Wilk, K. \& Perrault, M. (1995), "Images of occupations: Components of gender and status in occupational stereotypes". Sex roles, 32, 565-582.

Glunk, U. \& Olie, R. (2003), "Social integration of foreign newcomers in an international organization: A study of national identity salience and stereotyping over time". Paper presented at the 11th European Congress on Work and Organizational Psychology: Identity and Diversity in Organizations, Lisbon, Portugal

Goldin, C. \& Rouse, C. (2000), "Orchestrating Impartiality: The Impact of "Blind" Auditions on Female Musicians". American Economic Review, 90, 715-741.

Granovetter, M (1973), "The Strength of Weak Ties". American Journal of Sociology, $78,1360-1380$.

Greenwald, A. G., \& Banaji, M. R. (1995), "Implicit social cognition: Attitudes, self esteem, and stereotypes". Psychological Review, 102, 4-27.

Greenwald, A. G., McGhee, D. E. \& Schwartz, J. L. K. (1998), "Measuring individual differences in implicit cognition: The implicit association test". Journal of Personality and Social Psychology, 74, 1464-1480.

Haagsma, R. (1993), "Is Statistical Discrimination Socially Efficient?". Information Economics and Policy, 5, 31-50.

Haagsma, R. (1995), "Microeconomic models of gender discrimination in labour markets with asymmetric information". Unpublished Ph.D-thesis, University of Amsterdam.

Haber, S. E., Lamas, E. J. \& Green, G. (1983), “A New Method for Estimating Job Separations by Sex and Race". Monthly Labor Review, 106, 20-27.

Haessel, W. P. \& Palmer, J. W. (1978), "Market Power and Employment Discrimination". Journal of Human Resources, 13, 545-60.

Hamilton, D. L. \& Rose, T. L. (1980), "Illusory correlation and the maintenance of stereotypic beliefs". Journal of Personality and Social Psychology, 39, 832-845.

Hamilton, D. L. \& Trolier, T. K. (1986), "Stereotypes and stereotyping: An overview of the cognitive approach". In Dovidio, J. F. \& Gaertner, S. L. (eds.), Prejudice, discrimination, and racism. San Diego, CA, US: Academic Press, 127-163.

Hannan, R. L., Kagel, J. H., \& Moser, D. V. (2002), "Partial Gift Exchange in an Experimental Labor Market: Impact of Subject Population Differences, Productivity Differences, and Effort Requests on Behavior". Journal of Labor Economics, 20. 923-951.

Haslam, S. A., Oakes, P., \& Turner, J. C. (1996), "Social Identity, Self Categorization, and the Perceived Homogeneity of Ingroups and Outgroups: The interaction Between 
Social Motivation and Cognition". In Sorrentino, R. M. \& Higgins, E. T. (eds.), Handbook of Motivation and Cognition, Vol. 3: The Interpersonal Context, 182-222. Heath, Ch. \& Tversky, A. (1991), "Preference and Belief: Ambiguity and Competence in Choice under Uncertainty". Journal of Risk and Uncertainty, 4, 5-28.

Heckman, J. J. \& Siegelman, P. (1993), "The Urban Institute Audit Studies: Their Methods and Findings". In Fix, M. \& Struyk, R. J., (eds.), Clear and convincing evidence: Measurement of discrimination in America. Washington, D.C.: Urban Institute Press, 187-258.

Hellerstein, J. K., Neumark, D. \& Troske, K. R. (1997) "Market Forces and Sex Discrimination". National Bureau of Economic Research Working Paper: 6321.

Hertwig, R. \& Ortmann, A. (2001), "Experimental practices in economics: A methodological challenge for psychologists?". Behavioral and Brain Sciences 24, 383-451.

Hewstone, M. \& Lord, C. G. (1998), "Changing intergroup cognitions and intergroup cognitions and intergroup behavior: The role of typicality". In Sedikides, C., Schopler, J. et al. (eds.), Intergroup cognition and intergroup behavior. Mahwah, NJ, US: Lawrence Erlbaum Associates, 367-392.

Heywood, J. S. \& Peoples, J. H., Jr. (1994), "Deregulation and the Prevalence of Black Truck Drivers". Journal of Law and Economics, 37, 133-155.

Ho, J. L. Y., Keller, L. R. \& Keltyka, P. (2002), "Effects of Outcome and Probabilistic Ambiguity on Managerial Choices". Journal of Risk and Uncertainty, 24, 47-74.

Hoffman, C. \& Hurst, N. (1990), "Gender Stereotypes: Perception or Rationalization?". Journal of Personality and Social Psychology, 58, 197-208.

Hogg, M. A. (2001), "Social identity and the sovereignty of the group: A psychology of belonging". In Sedikides, C. \& Brewer, M. B. (eds.), Individual self, relational self. collective self. Philadelphia, PA, US: Psychology Press, 123-143.

Hogg, M. A. \& Hains, S. C. (1998), "Friendship and group identification: A new look at the role of cohesiveness in groupthink". European Journal of Social Psychology, 28, 323-341.

Hogg, M. A. \& Mullin, B. A. (1999), "Joining groups to reduce uncertainty: Subjective uncertainty reduction and group identification". In Abrams, D. \& Hogg, M. A. (eds.), Social identity and social cognition. Malden, MA, US: Blackwell Publishers, 249. 279.

http://www.ats.ucla.edu/stat/spss/faq/alpha.html, 11.04.2003 
http://www.fdewb.unimaas.nl/miso/index.htm, 11.04.2003

Hunt, S. (1997), "Women's Vital Voices", Foreign Affairs, July/August, 2-7.

Isen, A. M., Johnson, M. M., Mertz, E. \& Robinson, G. F. (1985), "The influence of positive affect on the unusualness of word associations". Journal of Personality and Social Psychology, 48, 1413-1426.

Isen, A. M., Daubman, K. A \& Nowicki, G. P. (1987), "Positive affect facilitates creative problem solving". Journal of Personality and Social Psychology, 52, 1122-1131.

Jetten, J., Spears, R., \& Manstead, A. S. R. (1996), "Intergroup norms and intergroup discrimination: Distinctive self categorization and social identity effects". Journal of Personality and Social Psychology, 71, 1222-1233.

Jetten, J., Spears, R., \& Manstead, A. S. R. (1997), "Distinctiveness threat and prototypicality: Combined effects on intergroup discrimination and collective self esteem". European Journal of Social Psychology, 27, 635-657.

Jetten, J., Spears, R., \& Manstead, A. S. R. (1998), "Defining dimensions of distinctiveness: Group variability makes a difference to differentiation". Journal of Personality and Social Psychology, 74, 1481-1492.

Jetten, J., Spears, R. \& Manstead, A. S. R. (1999), "Group distinctiveness and intergroup discrimination". In Ellemers, N., Spears, R. et al. (eds.), Social identity: Context, commitment, content. Oxford, England: Blackwell Science Ltd., 107-126.

Johnston, L. C. and C. N. Macrae (1994), "Changing Social Stereotypes: The Case of the Information Seeker". European Journal of Social Psychology, 24, 581-592.

Jost, J. T., \& Banaji, M. R. (1994), "The Role of Stereotyping in System Justification and the Production of False Consciousness". British Journal of Social Psychology, 33, 127.

Jowell, R. \& Prescott Clarke, P. (1995), "Racial Discrimination and White Collar Workers in Britain". In Darity, W. J. (ed.), Economics and discrimination. Volume 2, Elgar Reference Collection. International Library of Critical Writings in Economics, vol. 57, Cheltenham, U.K.: Elgar, 251-271.

Judd, Ch. M., Ryan, C. S., \& Park, B. (1991), “Accuracy in the judgment of ingroup and outgroup variability". Journal of Personality and Social Psychology, 61, 366-379. Jussim, L. (1991), "Social Perception and Social Reality: A Reflection Construction Model", Psychological Review, 98, 54-73. 
Jussim, L., M. Manis, et al. (1995), "Prejudice, Stereotypes, and Labeling Effects:

Sources of Bias in Person Perception". Journal of Personality and Social Psychology $68,228-246$.

Katz, L. F. (1986), "Efficiency Wage Theories: A Partial Evaluation”. In Fischer, S. (ed.) NBER Macroeconomics Annual. Cambridge, Mass.: MIT Press, 235-276.

Kinder, D. R. \& Sears, D. O. (1981), "Prejudice and politics: Symbolic racism versus racial threats to the good life". Journal of Personality and Social Psychology, 40, 414 431.

Klein, W.M. \& Kunda, Z. (1992), "Motivated Person Perception: Constructing Justifications for Desired Beliefs". Journal of Experimental Social Psychology, 28, 145-168.

Knight, F. H. (1921), Risk, Uncertainty and Profit. Boston: Houghton Mifflin.

Laibson, D. (1997), "Golden Eggs and Hyperbolic Discounting". Quarterly Journal of Economics, 112, 443-477.

Light, A. \& Ureta, M. (1992), "Panel Estimates of Male and Female Job Turnover Behavior: Can Female Nonquitters Be Identified?". Journal of Labor Economics, 10, 156-81.

Linville, P.W. \& Fisher, G.W. (1998), "Group variability and covariation: Effects on intergroup judgment and behavior". In Sedikides, C. \& Schopler, J. (eds.), Intergroup cognition and intergroup behavior. Mahwah, NJ, US: Lawrence Erlbaum Associates, 123-150.

Loewenstein, G. F., Weber, E. U., Hsee, C. K. \& Welch, N. (2001), "Risk as Feelings". Psychological Bulletin, 127, 267-286.

Lui, L. \& Brewer, M. B. (1983), "Recognition accuracy as evidence of category consistency effects in person memory". Social Cognition, 2, 89-107.

Luksetich, W. A. (1979), "Market Power and Sex Discrimination in White Collar Employment". Review of Social Economy, 37, 211-224.

Lundberg. Sh. J. (1991), "The Enforcement of Equal Opportunity Laws under Imperfect Information: Affirmative Action and Alternatives". Quarterly Journal of Economics, 106, 309-326.

Lundberg, Sh. J. \& Startz, R. (1983), „Private Discrimination and Social Intervention in Competitive Labor Markets". American Economic Review, 73, 340-347.

Mackie, D. M. \& Smith, E. R. (1998), "Intergroup relations: Insights from a theoretically integrative approach". Psychological Review, 105, 499-529. 
Macrae, C. N., Bodenhausen, G. V., Milner, A. B. \& Jetten, J. (1994), "Out of mind but back in sight: Stereotypes on the rebound". Journal of Personality and Social Psychology, 67, 808-817.

Major, B., McFarlin, D. B., \& Gagnon, D. (1984), "Overworked and Under Paid: On the nature of gender differences in personal entitlement". Journal of Personality and Social Psychology, 47, 1399-1412.

Mamuth, H. A. (1993), Markteconomie. Analyse en evaluatie. Utrecht.

Maslow, A. H. (1943), "A theory of Human Motivation". Psychological Review, 50, 370 -396 .

Mavromaras, K. G. \& Rudolph, H. (1999), "Occupational Segregation and the Male Female Wage Gap in Germany Between 1985 and 1995 ", presentation at the $I I^{\text {th }}$ EALE Anmual Conference.

Meng, Christoph (2002), "(fe)male jobs and (fe)male wages-disentangling the effect of personal and job characteristics on wages by measuring stereotypes". Paper submitted for publication.

Merton, R. K. (1948) "The bearing of empirical research upon the development of social theory". American Sociological Review, 13, 505-515.

Mullin, B. A., and Hogg, M. A. (1998), "Dimensions of subjective uncertainty in social identification and minimal intergroup discrimination". British Journal of Social Psychology, 37, 345-365.

Neal, D. A. \& Johnson, W. R. (1996), "The Role of Premarket Factors in Black White Wage Differences". Journal of Political Economy, 104, 869-895.

Nelson, Th. E.; Biernat, M. R. \& Manis, M. (1990), "Everyday base rates (sex stereotypes): Potent and resilient". Journal of Personality and Social Psychology. 59(4), 664-675

Neumark, D. and McLennen, M. (1995), "Sex Discrimination and Women's Labor Market Outcomes". The Journal of Human Resources 30, 713-739.

Nicholson, W. (1998), Microeconomic Theory; Basic Principles and Extensions. London: The Dryden Press.

Oaxaca, R. L. (1973), "Male-Female Wage Differentials in Urban Labor Markets". International Economic Review, 14, 673 - 709.

Oaxaca, R. L. \& Ransom, M. R. (1994), "On Discrimination and the Decomposition of Wage Differentials", Journal of Econometrics, 61, 5-21. 
O'Donoghue, T. \& Rabin, M. (1999), "Doing It Now or Later". American Economic Review, 89, 103-124.

O'Neill, J. (1985), "The Trend in the Male Female Wage Gap in the United States". Journal of Labor Economics, Part 2, 3, S91-116.

Oster, Sh. M. (1975), "Industry Differences in the Level of Discrimination against Women". Quarterly Journal of Economics, 89, 215-229.

Osterman, P. (1982), "Affirmative Action and Opportunity: A Study of Female Quit Rates". Review of Economics and Statistics, 64, 604-612.

Ottati, V. \& Lee, Y. (1995), “Accuracy: A Neglected Component of Stereotype Research". In Lee, Y., Jussim, L. J., \& McCauley, C. R. (eds.), Stereotype Accuracy. Washington, D.C.: APA, 29-59.

Hornby, A. S. (1987), Oxford advanced learners dictionary of current english. Oxford university press.

Paragon Educational Consulting Student Learning Style Inventory http://www.oswego.edu/ shindler/plsi/plsi48a.htm, 11.04.03

Pelham, B. W. \& Hetts, J. J. (2001), “Underworked and Overpaid: Elevated Entitlement in Men's Self Pay". Journal of Experimental Social Psychology, 37, 93-103.

Peoples, J. \& Saunders, L. (1993), "Trucking Deregulation and the Black/White Wage Gap". Industrial and Labor Relations Review, 47, 23-35.

Pettigrew, T. F. (1997), "Generalized Intergroup Contact Effects on Prejudice". Personality and Social Psychology Bulletin, 23, 173-185.

Petty, R. E. \& Cacioppo, J. T. (1981), Attitudes and Persuasion: Classic and contemporary approaches. Dubuque, IA: Wm C. Brown.

Phelps, E. S. (1972), "The Statistical Theory of Racism and Sexism". American Economic Review, 62, 659-661.

Pratto, F. \& Bargh, J. A. (1991), "Stereotyping Based on Apparently Individuating Infomation: Trait and Global Components of Sex Stereotypes under Attention Overload". Journal of Experimental Social Psychology, 27, 26-47.

Preston, I. \& Szymanski, S. (2000), "Racial Discrimination in English Football". Scottish Journal of Political Economy, 47, 342-363.

Quattrone, G. A. \& Jones, E. E. (1980), "The perception of variability within in groups and out groups: Implications for the law of small numbers". Journal of Personality and Social Psychology, 38, 141-152. 
Roethlisberger, F. J. \& Dickson, W. (1939), Management and the worker, Boston (Mass.).

Rothschild, K. W. (1981), Einfuehrung in die Ungleichgewichtstheorie. Berlin, Heidelberg, New York: Springer.

Sandell, S. H. \& Shapiro, D. (1978), “An Exchange: The Theory of Human Capital and the Earnings of Women: A Reexamination of the Evidence". Journal of Human Resources, 13, 103-117.

Savage, L. J. (1954). The Foundations of Statistics. New York: Wiley.

Schaller, M. \& Maass, A. (1989), "Illusory correlation and social categorization: Toward an integration of motivational and cognitive factors in stereotype formation". Journal of Personality and Social Psychology, 56, 709-721.

Schaller, M., Rosell, M. C., \& Asp, C. H. (1998), "Parsimony and Pluralism in the Psychological Study of Intergroup Processes". In Sedikides, C., Schopler, J. \& Insko, Ch. A. (eds.), Intergroup Cognition and Intergroup Behavior. Mahwah, NJ, US: Lawrence Erlbaum Associates, 3-25.

Schinkel, M. P. (2001), „Disequilibrium Theory. Reflections towards a Revival of Learning". Ph.D. thesis, Universiteit Maastricht.

Schwieren, C. (2002), "An erroneous belief - paying women lower wages does not pay!" Paper presented at the European ESA conference, Strasbourg.

Schwieren, C. \& Vendrik, M. (2002), "Psychological vs. economic effects of competition on discrimination: An integrated model". Mimeo, Universiteit Maastricht.

Schwieren, C., M. Vendrik and P. de Gijsel (2002), "The power of competition: Reducing or reinforcing discrimination?" In Stability and Dynamics of Power. Conference proceedings of IAREP/SABE conference 2002, Turku, 346 - 352.

Schwieren, C., \& Glunk, U., (2003), "National Identity as a Basis for Discrimination do Work Teams Prefer Hiring Ingroup Members?" Paper presented at the EAWOP conference, Lisbon.

Sedikides, C., Schopler, J. \& Insko, Ch. A. (eds.) (1998), Intergroup cognition and intergroup behavior. Mahwah, NJ, US: Lawrence Erlbaum Associates.

Sedikides, C., \& Brewer, M. B. (eds.) (2001), Individual Self, Relational Self, Collective Self, Philadelphia, Psychology Press.

Shenhav, Y. (1992), "Entrance of Blacks and Women Into Managerial Positions in Scientific and Engineering Occupations: A Longitudinal Analysis". Academy of Management Journal, 35, 889-901. 
Shepherd, W. G. \& Levin, Sh. G. (1973), "Managerial Discrimination in Large Firms". Review of Economics and Statistics, 55, 412-22.

Sherif, M., Harvey, O. J., White, B. J., Hood, W. R., \& Sherif, C. W. (1961), Intergroup conflict and cooperation: The Robbers Cave experiment. Norman: University of Oklahoma Press.

Sicherman, N. (1996), "Gender Differences in Departures from a Large Firm". Industrial and Labor Relations Review, 49, 484-505.

Singell, L. D. J. \& Thornton, J. (1997), "Nepotism, Discrimination, and the Persistence of Utility Maximizing, Owner Operated Firms". Southern Economic Journal 63, 904919.

Snyder, M. (1984), "When belief creates reality". In Berkowitz, L. (Ed.), Advances in experimental social psychology (18), New York: Academic Press, 248 - 206.

Snyder, M. (1992), "Motivational foundations of behavioral confirmation". In Zanna, M. P. (ed.), Advances in experimental social psychology (25), 67-114.

Snyder, M. \& Miene, P. (1994), "On the Functions of Stereotypes and Prejudice." In Zanna, M. P. \& Olson, J. M. (eds.), The Psychology of Prejudice: The Ontario Symposium, 55-89.

Spears, R. (2001), "The Interaction Between the Individual and the Collective Self". In Sedikides, C., \& Brewer, M. B. (eds.), Individual Self, Relational Self, Collective Self, Philadelphia: Psychology Press, 171-198.

Spears, R., Doosje, B. \& Ellemers, N. (1999), "Commitment and the context of social perception". In Ellemers, N., Spears, R. et al. (eds.), Social identity: Context, commitment, content. Oxford, England: Blackwell Science Ltd., 59-83.

Spears, R., Jetten, J. \& Doosje, B. (2001), "The (il)legitimacy of ingroup bias: From social reality to social resistance". In Major, B. \& Jost, J. (eds.) The Psychology of Legitimacy: Emerging Perspectives on Ideology, Justice, and Intergroup Relations. New York: Cambridge University Press, 332-363.

Stangor, C., Sullivan, L. A. et al. (1991), "Affective and Cognitive Determinants of Prejudice". Social Cognition 9, 359-380.

Stanley, T. D. \& Jarell, S. B. (1998), "Gender Wage Discrimination Bias? A Meta Regression Analysis". The Journal of Human Resources, 33, 947-73.

Stigler, G. J. \& Becker, G. S. (1977), "De Gustibus Non Est Disputandum". American Economic Review, 67, 76-90. 
Stroebe, W., Hewstone, M., \& Stephenson, G. M. (1996), Sozialpsychologie. Eine Einfuehrung. Berlin, Heidelberg, New York: Springer.

Szymanski, S. (2000), "A Market Test for Discrimination in the English Professional Soccer Leagues". Journal of Political Economy, 108, 590-603.

Tajfel, H., Billig, M. G., Bundy, R. P. \& Flament, C. (1971), "Social categorization and intergroup behaviour". European Journal of Social Psychology, 1, 149-178.

Tetlock, P. E. (1992), "The Impact of Accountability on Judgment and Choice: Toward a Social Contingency Model". In Zanna, M. P. (ed.), Advances in Experimental Social Psychology, 25, 331-377.

Tiedens, L. Z. \& Linton, S. (2001), "Judgment Under Emotional Certainty and Uncertainty: The Effects of Specific Emotions on Information Processing". Journal of Personality and Social Psychology, 81, 973-988.

Turner, J. C. (1981), "Towards a cognitive redefinition of the social group". Cahiers de Psychologie Cognitive/Current Psychology of Cognition, 1, 93-118.

Turner, J. C. (1999), "Some Current Issues in Research on Social Identity and Selfcategorization Theories". In Ellemers, N., Spears, R., \& Doosje, B. (eds.), Social identity: Context, commitment, content. Oxford, England: Blackwell Science Ltd., 11-46.

Turner, J. C. \& Oakes, P. J. (1986), “The Significance of the Social Identity Concept for Social Psychology with Reference to Individualism, Interactionism and Social Influence". British Journal of Social Psychology, 25, 237-252.

Van de Vliert, E. (1999), "Cooperation and Competition as Partners". European Review of Social Psychology, 10, 231-257.

van Dijk, F., Sonnemans, J. \& van Winden, F. (2002), "Social Ties in a Public Good Experiment". Journal of Public Economics, 85, 275-299.

Viscusi, W. K. (1980), "Sex Differences in Worker Quitting". Review of Economics and Statistics, 62, 388-398.

Viscusi, W. K. \& Chesson, H. (1999), "Hopes and Fears: The Conflicting Effects of Risk Ambiguity". Theory and Decision, 47, 153-178.

Von Neumann, J. \& Morgenstern, O. (1947), Theory of Games and Economic Behavior. $2^{\text {nd }}$ ed. Princeton: Princeton University Press.

Walters, A. E., Stuhimacher, A. F., \& Meyer, L. L. (1998), "Gender and Negotiator Competitiveness: A Meta analysis". Organizational Behavior and Human Decision Processes, 76, 1-29. 
References

Wells, G. L. \& Windschitl, P. D. (1999), "Stimulus sampling and social psychological experimentation". Personality and Social Psychology Bulletin, 25, 1115-1125.

Yzerbyt, V. Y., G. Schadron, et al. (1994), "Social Judgeability: The Impact of Meta Informational Cues on the Use of Stereotypes". Journal of Personality and Social Psychology, 66, 48-55.

Zafirofsky, M. (2002), "Reconsidering equilibrium: a socio economic perspective". The Journal of Socio Economics, 31, 559-579. 


\section{Author Index}

\section{A}

Aigner, D. . . . . . . . . . . . . 27

Akerlof, G. . . . . . . 13, 38, 39

Allport, G. . . . . . . . . . 26, 33

Altonij, J. . . . . . . . . . . 20

Altonji, J. . . . . . . . . . . 65

Amir, Y................ 26

Anderson, D. . . . . . . . . . . 17

Anshel, M. . . . . . . . . . . . 49

Arrow, K.......... 11, 12, 19, 27, 28, $32,63,177,196$

Ashenfelter, O. ........ 19,47

Ashraf, J...................................... 17, 18

\section{B}

Baetge, J........... 150, 151

Baldwin, M. .............. 20

Ball, S. ............................................... 179

Banaji, M. .... 24, 25, 29, 34, 51, 212

Bargh, J. . . . . . . . . . . . . 24

Baron, J. . . . . . . ......... 41

Becker, G. . . ...... 11-13, 19, 20, 31-

33,

46-55, 63-67, 177, 194, 213, 219

Beller, A.................................................. 18

Bergmann, B. . . . . . . . . 32

Berk, J. . . . . . . . . . . . . 27

Bertrand, M......................................... 18

Betsch, T. . . . . . . . . . . . . 29

Biernat, M. . . . . . . 23, 29

Black, D. ................................................ 48

Blank, R. . . . . . . . . 20,65

Blau, F.....9. 10, 17-20, 27, 32, 178, 195

Blaug, M. . . . . .

Blinder, A. ........................................... 17

Bloch, F. . . . . . . . . . . . 38

Bodenhausen, G........ 23 - 30, 33

Borjas, G. ............................................... 48

Bornstein, G.............. 49
Bowlus, A................................................ 48

Brandts, J. . . . . . . . . . . . . 9 9

Brewer, M. . . . 15, 23, 33, 37, 219

Brickenkamp, R............ 101

Brigham, J. . . . . . . . . . . . 33

Bronars, S. .............................................. 48

Bruggert, F............... 20

C

Cacioppo .................................................... 29

Cain, G................... 27

Camerer, C..... 40-44, 51, 108, 212

Carmichael, H. ....................................... 177

Cattell, R. . . . . . . . . . . 101

Chaiken, S........................ 12, 29, 94, 99

Charness, G. . . . . . . . . . 9, 179

Chesson, H. . . . . . . . . . . 40 40

Claire, Th. ................ 23

Clore, G. . . . ............... 29

Coate, S. ........ 12, 27, 66, 196

Comanor, W......... 11, 47, 64

Conway, M................ 29

Cook, S.................. 26

Corcoran, M. ............................................ 18

D

Damasio, A.......... 24, 29, 33

Darity, W. . . . . . . . . . . 32

Darley, J. . . . . . . . . . 23, 33

Datta Gupta, N. ...................................... 17

de Gijsel, P................................................ 89

Deaux, K. ............................................... 178

Dekker, H. . . . . . . . . 147, 163

Dequech, D................ 41

Dickson, W............... 163

Dijker, A................. 24

Doosje, B. . . . . . . . . . 35, 37

Dovidio, J......... 25, 29, 33, 34

Dovodio, J. . . . . . . . . . . . . 34

Duncan, G. ............................................. 18 
E

Eagly, A.

$12,29,94,99$

Eckel, C................................................ 179

Eckstein, Z. ............................................. 48

Ellemers, N. . . . . . . . . 35, 37

Ellsberg, D............ 40,41

Ely, R................. 38

Esses, V................ 24

F

Falk, A.

179-185

Farmer, A. . . . . . . 12, 27, 196

Fazio, R. . . . . . . . 23, 33, 34

Fehr, E. ........... 9, 179-185

Ferber, M. ................................... 18, 195

Festinger, L. . . . . . . . . . 28

Fiedler, K... 21, 22, 27, 36, 64, 67, 68, 110,111

Fischbacher, U. . . . . . 110, 182

Fischer, Th. ........... 150, 151

Fisher, $G$. ..... 10, 21, 22, 24, 25, 34

Fiske, S. . ...16, 21-6, 32, 63, 177, 179, 213

Fortin, N............................................... 17

Fox, C. ............... 41

Friedman, D. ......... 34, 45, 56

Frisch, D. . . . . . . . . . . . 41

Fuchs, V.................................................... 17

Fujii, E................. 19

$\boldsymbol{G}$

Gaertner, S............ 25, 33

Gagnon, D. . . . . . . . . . . . 195

Gardner, R. . . . . . . . . . . . 21

Garst, J................... 33

Gigerenzer, G.............. 9

Glick, P. ................................................ 178

Glunk, U. ...................................... 144, 145

Goldin, C. ................................................ 19

Granovetter, M.............. 9

Green, G................. 20

Greenwald, A. . ...25, 29, 51, 196, 212, 218

Grossman, P.
$\boldsymbol{H}$

Haagsma, R. . . . . . . . 66, 177

Haber, S. . . . . . . . . . . . 20

Haddock, G. . . . . . . . . . . 24

Haessel, W. . . . . . . . . . . . 19

Hains, S. . . . . . . . . . . . 36

Hallock, K................................................ 18

Hamilton, D. . . . . . 10, 21-3, 33

Hannan, T........... 19, 47, 179

Haslam, A.............. 34-36

Heath, Ch. . . . . . . . . . . 41

Heckman, J.............................................. 19

Hellerstein, J.. . . . . . . . . . . 20

Hertwig, R. ......................................56, 90

Hetts, J................................................... 178

Hewstone, M. . . . . . . . . 26

Heywood, J................. 19

Ho, J.................. 40

Heffman $C$............... 34

Hogg, M. . . . 11, 35-37, 42, 43, 49, 50, $63,102,153,218$

Hunt, S. . . . . . . . . . . . 62

Hurst, N. .............. 34

I

Isen, A. . . . . . . . . . . . 24

$\boldsymbol{J}$

Jamieson, J. . . . . . . . . . . . 49

Jarell, S.......................................... 17, 177

Jarrell, S. ...................................... 19, 55

Jetten, J.. . . . . . . . . . . . . . 37

Johnson, W........... 20, 27, 33

Johnston, L.................. 26

Jones, E. . . . . . . . . . . . . 22

Jost, J. . . . . . . . . . . . 24, 34

Jowell, R_.................................................... 19

Judd, Ch................. 22

Jussim, L. . . . . . . . 26, 29, 33

$\boldsymbol{K}$

Kagel 179 
Kahn, L. . . . . . . 9, 17-20, 178

Katz, L. ................................................ 177

Kinder, D....................

Kirchsteiger, G............. 181

Klein, W................... 24

Knight, F. ............40, 212

Kobrynowic, D. . . . . . . . . . 23

Koomen, W................. 24

Kranton, R............. 13, 38

Kunberger, A.......... 150, 151

Kunda, Z. ................ 24

$\boldsymbol{L}$

LaFrance, M.............................................. 178

Laibson, D. . . . . . . . . . . . . . 9

Lamas, E. ................. 20

Lee, $\mathrm{Y}, \ldots \ldots \ldots \ldots \ldots \ldots \ldots, 22$

Lemieux, T....................................................... 17

Levin, Sh. ................. 20

Light, A. . . . . . . . . . 20, 178

Liwivi, s. ..................... 30

Linville, P. . . . . . 21-5, 34

Loewenstein, G. ............. 29

Lord, C.................. 26,

Loury, G.......... 12, 27, 66, 196

Lui, L.................... 23

Luksetich, W. . . . . . . . . . 19

Lundberg, Sh. .............. 27

M

Maass, A. ................. 34

Mackie, D. .................. 33

Macrae, C............ 25, 26, 33

Major, B.................. 195

Mamuth, H................ 52

Manis, M. ................. 29

Manstead, A................ 37

Maslow, A...................

Mavromaras, K. ...................................... 17

McFarlin, D. . . . . . . . . . . 195

McLennen, M. ....................................... 19

Meng, Ch. ................. 195

Merton, R............... 23, 33

Meyer, L. .................................................. 178
Miene, P................... 24

Moreno, K. ................. 30

Morgenstern, $\mathrm{O} . \ldots \ldots \ldots \ldots \ldots \ldots, 40$

Moser ….............................................. 179

Mullin, B.. . . 11, 35-37, 42, 43, 49, 50, $63,102,153,218$

N

Neal, D.................... 27

Nelson, Th. .................. 29

Neumark, D. ............................................... 19

Nicholson, W............... 45

Nickel, S.................. 68

$\boldsymbol{o}$

O'Donoghue, T. . . . . . . . . . 9

Oaxaca, R...................................................... 17

Olie, R. .................. 145

O'Neill, J. ......................................17, 18

Ortmann, A .......................................56, 99

Usier, Sh....................... 20

Osterman, P. . . . . . . . . . . . . 20

Ottati, V.................. 22

$\boldsymbol{P}$

Palmer, J.................. 19

Park, B................... 22

Pelham, B. ................................................ 178

Peoples, J................. 19,

Pettigrew, T. ....... 29, 33, 51, 212

Petty ...........................................................29

Phelps, E........... 11, 19, 27, 177

Pierret, C. . . . . . . . . . . . . 20

Pratto, F................... 24

Prescott-Clarke, P....................................... 19

Preston, I. . . . . . . . . . . . . 20

$Q$

Quattrone, G................ 22

$\boldsymbol{R}$

Rabin, M.................. 9

Ransom, M. ...................................................... 17

Rao, V. ................... 38 
Raviv, Sh. . . . . . . . . . . 49

Riedl, A. ................ 181

Roethlisberger, F. . . . . . . . 163

Rose, T.................. 23

Rothschild, K..... 39, 42, 46, 53, 59

Rouse, C................................................ 19

Rudolph, H.......................................... 17

Ryan, C. . . . . . . . . . . 22

$\boldsymbol{S}$

Sandell, S. .............................................. 18

Saunders, L. . . . . . . . . . . . 19

Savage, L. . . . . . . . . . . . 40

Schaller, M. .......... 29, 34, 64

Schinkel, M.-P.............. 12

Schippers. . . . . . . . . . . . . . 20

Schmidt, K................ 9

Schwarz, N. . . . . . . . . . 29, 36

Sears, D. . . . . . . . . . . . . . .

Selten, R................. 9

Shapiro, D. ....................................... 17, 18

Shenhav, Y.................................... 17, 18

Shepherd, W. . . . . . . . . . . 20

Sherif, M. . . . . . . . . . 49, 59

Sicherman, N. . . . . . . . . 20, 178

Siegelman, P. ....................................... 19

Singell, L................................................. 55

Smith, E. .................. 34

Snyder, M. . . . . . . . . 23, 24

Sonnemans, J. . . . . . . . . . . . . 9

Spears, R. . . . . . . 35-37, 118, 127

Stangor, C. . . . . . . . . . . . 29

Stanley, T..................... 17, 19, 55, 177

Startz, R.................. 27

Stevens, L. . . . . . . . . . . . . 26

Stigler, G. . . . . . . . . . . . . 63

Stroebe, W................. 38
Stuhlmacher, A....................................... 178

Sunder, Sh. . . . . . . . 34, 45, 56

Szymanski, S. . . .......... 20

$\boldsymbol{T}$

Tajfel, H. . . . 21, 22, 35, 37, 101

Terrell, D. . . . . . . . 12, 27, 196

Tetlock, P................ 23

Thomton, J. ............................................. 55

Tiedens, L................ 36

Trapani, J. . . . . . . . . . . . . . 19

Trolier, T. . . . . . 10, 21, 22, 33

Turner, J................ 34-37

Tversky, A. ............. 41

$\boldsymbol{U}$

Ureta, M. . . . . . . . 20, 178

$\boldsymbol{V}$

v. Neumann, J............. 40

Van de Vliert, E. ..............4 49

van Dijk, F................ 9

van Winden, F. . . . . . . . . . 9

Vendrik, M. . . . . . . . . . 62,89

Viscusi, W. . . . . . . . 20, 40, 178

$\boldsymbol{W}$

Walters, A. …….................................... 178

Walther, E. . . . . . . . . . . . 68,

Weber, M. . . 40, 41, 44, 51, 108, 212

Weiss, R................ 101

Wyer, R. . . . . . . . . . . . . 23

$\boldsymbol{Z}$

Zafirofsky, M.......... 44,46

Zame, W........................................... 179

Zanna, M. ................ 24 


\section{Subject Index}

B

behavior......... 9-16, 20 - 39, 43- 63, $68,89-99,108,110,113,118-126$, $144-147,162-177,178,182,193-196$, 211-219

C

cognitions ....... 13, 24, 34, 38, 45, cognitive.........9, 13, 16, 22-38, 56, 63, $86,87,101,108,196,219$ competition..... . 11-16, 25, 41-66, 80, $86-113,118,119,123-133,144,148-$ $150,155,159-167,170,174,196$, 211-219

competitive ........11-14, 31, 45-66, 86, $89-91,103,119,150,164,178,179$. $196,213,218$

\section{D}

decisions . ..........10-12, 27-33, 36, 40, $63-68,109,112,151,165,173,213$, 216

discrimination. . ...9-33, 37-66, 85-98, $101-111,119,125-128,144-56,162-$ $167,177,194,211-219$ discriminatory taste....... 11, 12, 32, 38, $47,53,63,65,85,147,148$

E

econometric. ........ 9, 17, 18, 55 economics . ........9-15, 20, 26-34, 38, $39,44-57,90,144,177,180,96,211$ efficiency-wage effects..........14, 65, 66, 178,215 emotional........13, 16, 24, 29-38, 42-45, $51,63,66,68,86,87,94,99,146$, $147,195,196,212,216-219$ equilibrium . $13,32,39-54,66,80$, experiment. $9,12,14,20,35-37$, $55-59,86,89-127,135,141-145,150$ $154,166,178-182,193-197,204$, 213-219

experimental methods 12

G

group information 11

$\boldsymbol{H}$

hiring. $10-12,19,25-29,47,54$, $55,63-68,86,91,98,112,117,165$, $166,213,216,218$

\section{I}

identification....... 11-13, 22, 35-39, 43, 49-54, 59, 62-64, 86, 87, 91, 99-109. $112-119,123-128,143,144,148,150$, $153,160,161,164,165,211-219$

imperfect information...........11, 27, 39, $42,46-50,54,112,146$

income effect....... 11, 47, 64, 213 integrated model..........13, 16, 62-66, $86-93,109,146,150,195,196,212$ 219

integration....... 9, 10-16, 22, 33, 34, $52-54,58,62,65,196,211,215,219$

\section{$\boldsymbol{L}$}

labor market ...2, 9-20, 31, 37, 46, 52 $62,66,67,86,89,126,178,180,196$ $-198,204,205,211-213,216,219$

\section{M}

methods.....9, 12, 18, 55-58, 118, 145, 177

N

neoclassical . . . . . 11,41-45,49 
social identity theory ..... 11, 91, 212 social psychology...... 9-16, 21, 22, 28, $29,33,34,38-44,52-59,62-67,177$, $179,211-215,219$

statistical discrimination..... 11, 20, 27, $53,65,109,119,127,146,148,216$

stereotypes........12, 16, 21-36, 51, 54 $58-68,80,85-94,99,116,119,124$, $126,144-150,161-163,166,177-180$, 194, 211-219

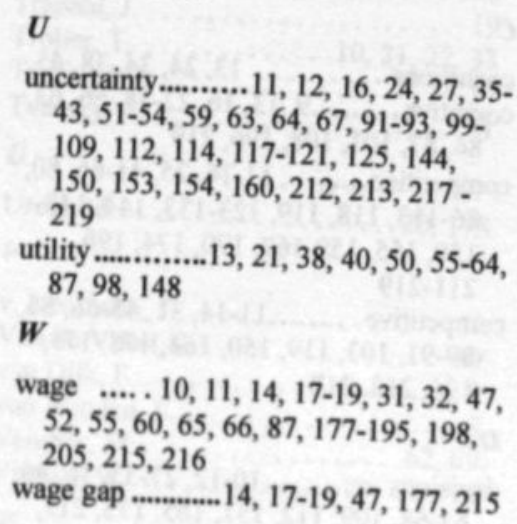
$11,12,16,24,27,35$ $43,51-54,59,63,64,67,91-93,99$ $109,112,114,117-121,125,144$, $150,153,154,160,212,213,217$. 219 


\section{Summary}

This thesis develops an interdisciplinary approach to discrimination of various groups (women, national groups etc.) in the labor market. The main aims of the research are: (i) To get an overview of literature in economics and social psychology relevant for an analysis of discrimination in the labor market, (ii) to develop an integrated, testable theoretical model making use of both literatures, and (iii) to test at least part of the predictions of this model experimentally. The basic questions to be answered thus are, whether an integration of economic and psychological factors can explain discriminatory behavior on the labor market better than one approach alone; whether an integrated theoretical model can lead to new testable predictions; and finally, whether experimental tests can provide evidence for at least some of these predictions. More in particular, a model is constructed which, by inclusion of the psychological concepts of prejudice, stereotypes, and identification, is able to explain and predict how discriminatory preferences may develop during a process of increasing competition. Predictions of this model are tested in experimental and quasi-experimental studies.

Chapter 2 provides an overview of some related literature on discrimination in economics and social psychology. First, empirical evidence on discrimination in the labor market is reviewed. It is shown that definitions of the core concepts differ within and between the disciplines, but also that there are some important similarities between them. Finally, an integrative framework is described which provides a starting point for the development of a formal integrated model focusing on some specific aspects of the framework. Some methodological questions with respect to the approach of the research to be conducted are discussed at the end of the chapter.

Chapter 3 develops the integrated model formally. Social-psychological and economic insights are used to develop a model that explains how an increase in competition can induce employers to rely more on stereotypic perceptions and prejudices in their hiring, pay and promotion decisions. This is based on the observation in social psychology that using stereotypic perceptions (for example, in personnel decisions) has certain benefits as well as costs (e.g., Fiske 1998). The most important benefit in this context is that, together with identification with an ingroup (e.g., men), the use of stereotypes and prejudices reduces subjective feelings of uncertainty, arising from competition. This psychological effect of strengthening competition works in the 


\section{Nederlandse samenvatting}

Dit proefschrift ontwikkelt een interdisciplinaire benadering voor de studie van discriminatie van verschillende groepen (vrouwen, nationaliteiten etc.) op de arbeidsmarkt. De hoofddoelen van het onderzoek zijn (i) een overzicht te verkrijgen van de relevante literatuur in de economische wetenschappen en de sociale psychologie voor een analyse van discriminatie op de arbeidsmarkt, (ii) een theoretisch model te ontwikkelen dat de kennis uit beide wetenschappen integreert en dat vervolgens kan worden getest, en (iii) het experimenteel testen van een gedeelte van de verklarende variabelen van het model. De te beantwoorden basisvragen zijn of de integratie van economische en psychologische factoren discriminerend gedrag op de arbeidsmarkt beter kan verklaren dan een van de twee benaderingen afzonderlijk, of een geîntegreerd theoretisch model tot nieuwe, toetsbare hypothesen leidt, en tenslotte, of experimentele toetsen deze nieuwe hypothesen bevestigen. Er wordt een model geconstrueerd dat, door integratie van psychologische concepten zoals vooroordelen, stereotypen en identificatie, verklaart en voorspelt hoe zich discriminerende voorkeuren kunnen ontwikkelen tijdens een proces van toenemende concurrentie. De hypothesen van het model worden middels experimentele en quasi-experimentele onderzoeken getest.

Hoofdstuk 2 geeft een overzicht van de literatuur op het gebied van discriminatie in de economie en de sociale psychologie. Eerst wordt een overzicht gegeven van empirische feiten over discriminatie op de arbeidsmarkt. Verder laat het hoofdstuk zien dat de definities van de belangrijkste concepten niet alleen tussen de disciplines, maar ook binnen de disciplines verschillen. Echter, op sommige belangrijke punten lijken de concepten op elkaar. Tenslotte wordt er een geïntegreerd kader beschreven, dat als uitgangspunt dient voor het ontwikkelen van een geformaliseerd en geĭntegreerd model gericht op sommige specifieke aspecten binnen dit kader. Verder komt aan het einde van het hoofdstuk een aantal methodologische vragen aan bod met betrekking tot de onderzoeksmethode zoals toegepast in dit proefschrift.

Hoofdstuk 3 ontwikkelt het geintegreerde model formeel. Sociaal-psychologische en economische inzichten worden gebruikt om een model te ontwikkelen dat verklaart hoe een toename in concurrentie ertoe kan leiden dat werkgevers in hun beslissingen over aanstelling, betaling en promotie van werknemers steeds meer gebruik maken van stereotype percepties en vooroordelen. Dit is gebaseerd op een waarneming uit de sociale psychologie, waarbij het gebruik van stereotype percepties (bijvoorbeeld bij beslissingen 
over personeel) bepaalde kosten en baten heeft (bijvoorbeeld Fiske, 1998). Het belangrijkste nut in deze context is dat het gebruik van stereotypen en vooroordelen samen met de identificatie met een 'ingroup' (bijvoorbeeld mannen) de subjectieve gevoelens van onzekerheid die door concurrentie ontstaan reduceert. Dit psychologische effect van versterkte concurrentie werkt in tegengestelde richting van het economische inkomenseffect in het model van werkgeversdiscriminatie van Becker (1957). Beide effecten zijn geintegreerd in het model. Het model kan ondanks of vanwege concurrentie de persistentie en de versterking van discriminatie op de arbeidsmarkt verklaren. Dit wordt bereikt door de introductie van stereotypen en vooroordelen als endogene variabelen. In de geanalyseerde situatie zijn de bedrijven in teams gestructureerd, zodat het model discriminatie door de werkgever en door de werknemer in een dergelijke situatie kan verklaren. De vraag in welke situatie welk van deze twee effecten, het 'psychologische' of het 'economische', sterker is, is een empirische vraag en blijft open.

Hoofdstuk 4 is een eerste stap richting het empirisch toetsen van een gedeelte van het geïntegreerde model. In eerste instantie worden alleen maar de psychologische voorspellingen voor verschillende maten van concurrentie getoetst. De experimenten met artificiele categorien bewijzen dat, afhankelijk van de mate van concurrentie, psychologische mechanismen 'discriminerend' gedrag beĩnvloeden zoals door het geĭntegreerde model was voorspeld. De experimenten laten verder zien dat een effect van toenemende concurrentie is, dat personen gebruik maken van alle 'informatie' die er is tenminste, wanneer ze veronderstellen dat groepslidmaatschap alleen al enige informatie is. Alleen bij sterke concurrentie vindt er statistische discriminatie plaats door de proefpersonen tegen de groep van wie men denkt dat ze gemiddeld slechter is. Dat is een zwakke variant van 'statistische discriminatie', omdat groepslidmaatschap in feite geen aan de productiviteit gerelateerde informatie is.

Hoofdstuk 5 breidt de empirische toets uit naar een complexere situatie, daarbij gebruik makend van een management simulatiespel met echte categorieên: verschillende nationaliteiten (Nederlands en Duits). De resultaten van deze studie bevestigen het idee dat psychologische effecten bepalend zijn voor het gedrag in een beperkte 'economische' situatie. Wij vinden een ingroup 'bias', maar geen 'bias' ten gunste van Duitse outgroup leden. Vanuit een productiviteitsgerelateerd oogpunt is de afwezigheid van vertekening 'irrationeel' gedrag, omdat Duitse studenten Bedrijfskunde aan de Universiteit Maastricht gemiddeld beter presteren dan Nederlandse studenten - en zo worden ze ook gezien. Echter, de nationaliteit van de groep die de beslissing neemt speelt een 
belangrijkere rol en heeft meer effect: Duitse groepen zijn eerder bereid om een nieuw lid op te nemen in hun groep dan Nederlandse groepen. Daarbij maakt het niet uit of het nieuwe lid tot de ingroup of de outgroup behoort. Versterking van concurrentie leidt tot meer ingroup 'bias' en meer 'bias' in het nadeel van Duitse nieuwe leden (de beter presterende), maar niet van Nederlandse nieuwe leden (de minder presterende). Het onderzoek geeft een indicatie dat de psychologische mechanismen, zoals door het geintegreerde model voorgesteld, wel degelijk een rol spelen bij het bepalen van voorkeuren voor nieuwe teamleden. Deze kunnen zelfs sterker zijn dan economische mechanismen.

Hoofdstuk 6 kijkt naar een iets ander aspect van loondiscriminatie. Om te testen of het efficientielooneffect het loonverschil tussen mannen en vrouwen beǐnvloedt, wordt een experimentele dubbele veiling gehouden. Het experiment laat zien dat zelfs in een dubbele veiling in het laboratorium vrouwen minder verdienen dan mannen. Echter, de invloed van efficiēntielooneffecten op dit resultaat kan niet helemaal worden verklaard. Vrouwen spannen zich in het experiment nooit sterk in, maar zij krijgen ook nooit een hoog loon aangeboden. Het is daarom niet duidelijk of zij altijd minder zouden reciproceren dan mannen, of dat mannen (en vrouwen!) juist verwachten dat vrouwen zich al voor een lager loon sterker inspannen, of dat ze zich aberhaupt nooit sterk inspannen. Duidelijk is dat vrouwen niet te bescheiden zijn, i.e. ze vragen geen lager loon. Tot slot wijst het experiment naast het absolute loon op een nieuwe verklarende factor voor verschillen in inspanning: het verschil tussen het loon door de werknemer gevraagd en het loon aangeboden door het bedrijf. Wanneer een werknemer een veel lager loon door de werkgever krijgt aangeboden dan hij gevraagd heeft, dan wordt dat gezien als een oneerlijke actie van de werkgever en kan dit leiden tot minder inspanning. Dit resultaat geeft een indicatie van een mogelijke vicieuze cirkel: vrouwen krijgen een loon aangeboden dat lager is dan zij hebben gevraagd en als gevolg spannen ze zich minder in. Werkgevers zien hun stereotypen bevestigd en, zoals gebeurd in het experiment, stoppen met het aanstellen van vrouwen uit louter rationele overwegingen. Dat zou ook het economische effect van concurrentie kunnen tegenwerken zoals voorgesteld bij Becker (1957), omdat daar wordt aangenomen dat dit effect middels lagere lonen voor de vrouwen in beginperiodes werkt.

Hoofdstuk 7 vat de resultaten van alle hoofdstukken samen en bediscussieert ze in een bredere context. Er wordt nagegaan of het gelukt is om de basisvragen van het onderzoeksproject te beantwoorden en of er nieuwe vragen zijn ontstaan. Hoewel vele 
vragen onbeantwoord zijn gebleven en er onvoldoende empirische resultaten zijn voor de formulering van gefundeerde beleidsadviezen ter bestrijding van discriminatie op de arbeidsmarkt, kan worden geconcludeerd, dat interdisciplinair onderzoek vruchtbaar kan zijn voor de analyse van dit onderwerp. Meer onderzoek is nodig, maar de tot nu toe bereikte resultaten zijn veelbelovend en laten de verwachting toe dat goed gefundeerd advies aan de beleidsmakers vanuit een dergelijke benadering kan voortkomen. Het onderzoek heeft verder laten zien dat, hoewel er altijd problemen zijn, interdisciplinair onderzoek kan bijdragen tot de ontwikkeling van beide disciplines. 


\section{About the author}

Christiane Schwieren was born on January $28^{\text {th }}$, 1972 in Duesseldorf, Germany. From 1991 to 1999, she studied psychology and political science at the University of Heidelberg. In 1997 she received her degree (M.A.) in political science, and in 1999 she received her degree (diploma) in psychology. During her study she worked as a student assistant at the department of social psychology, carrying out experimental research on value judgments. After finishing her studies, she started her $\mathrm{PhD}$ research at the University of Maastricht in September 1999, which she finished in September 2003. Her research on integrative approaches to discrimination on the labor market was supervised by Prof. Peter de Gijsel and Dr. Maarten Vendrik. For her PhD research she spent half a year as a Maric-Curie pro-doctoral fellow at the Universitat Autonoma in Barcelona. From September 2003 on she works as an assistant professor at Universitat Pompeu Fabra in Barcelona. 



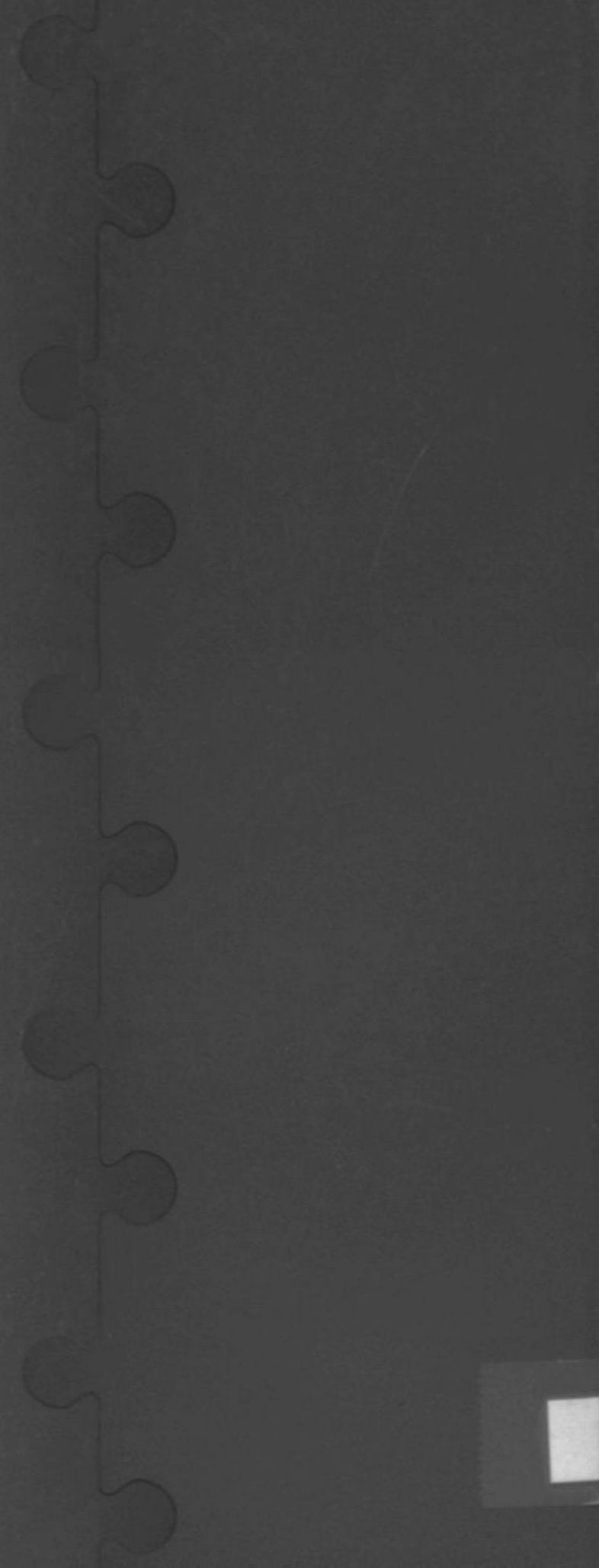

